Prepared for the U.S. Department of Energy

Under Contract DE-AC05-76RL01830

\title{
EFRT M-12 Issue Resolution: Comparison of Filter Performance at PEP and CUF Scale
}

RC Daniel

JM Billing

JR Bontha

CF Brown

August 2009

\author{
PW Eslinger \\ BD Hanson \\ JL Huckaby \\ NK Karri
}

ML Kimura

DE Kurath

MJ Minette 


\title{
DISCLAIMER
}

This report was prepared as an account of work sponsored by an agency of the United States Government. Neither the United States Government nor any agency thereof, nor Battelle Memorial Institute, nor any of their employees, makes any warranty, express or implied, or assumes any legal liability or responsibility for the accuracy, completeness, or usefulness of any information, apparatus, product, or process disclosed, or represents that its use would not infringe privately owned rights. Reference herein to any specific commercial product, process, or service by trade name, trademark, manufacturer, or otherwise does not necessarily constitute or imply its endorsement, recommendation, or favoring by the United States Government or any agency thereof, or Battelle Memorial Institute. The views and opinions of authors expressed herein do not necessarily state or reflect those of the United States Government or any agency thereof.

\author{
PACIFIC NORTHWEST NATIONAL LABORATORY \\ operated by \\ BATTELLE \\ for the
}

UNITED STATES DEPARTMENT OF ENERGY

under Contract DE-AC05-76RL01830

Printed in the United States of America

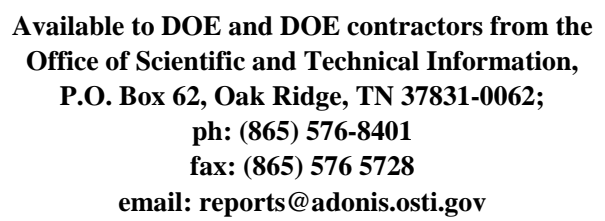

Available to the public from the National Technical Information Service, U.S. Department of Commerce, 5285 Port Royal Rd., Springfield, VA 22161 ph: (800) 553-6847 fax: (703) 605-6900

email: orders@nits.fedworld.gov

online ordering: http://www.ntis.gov/ordering.htm 


\section{EFRT M-12 Issue Resolution: Comparison of Filter Performance at PEP and CUF Scale}

$\begin{array}{lll}\text { RC Daniel } & \text { PW Eslinger } & \text { ML Kimura } \\ \text { JM Billing } & \text { BD Hanson } & \text { DE Kurath } \\ \text { JR Bontha } & \text { JL Huckaby } & \text { MJ Minette } \\ \text { CF Brown } & \text { NK Karri } & \end{array}$

August 2009

Test specification: 24590-PTF-TSP-RT-07-001 Rev 2

Work Authorization: WA\# 2007-024

Test plan: TP-RPP-WTP-506, Rev. 0.4; TP-WTP-PEP-044, Rev. 0.2

Test exceptions: $\quad$ 24590-PTF-TEF-RT-08-00002

24590-WTP-TEF-RT-09-00003

24590-PTF-TEF-RT-09-00001

24590-WTP-TEF-RT-09-00002, Rev. 0

24590-WTP-TEF-RT-09-00001 Rev. 1

R\&T focus area: Pretreatment

Test Scoping Statement(s): NA

Prepared for

the U.S. Department of Energy

Under Contract DE-AC05-76RL01830

Pacific Northwest National Laboratory

Richland, Washington 99352 


\section{COMPLETENESS OF TESTING}

This report describes the results of work and testing specified by Test Specification 24590-PTF-TSP-RT-07-001 Rev 2 "Pretreatment Engineering Platform (PEP) Testing (Phase 1)" and Test Plans TP-RPP-WTP-506 Rev 0.4 "Pretreatment Engineering Platform (PEP) Testing (Phase 1) and TP-WTP-PEP-044 Rev 0.2" Test Plan for the PEP Parallel Laboratory Testing." The work and any associated testing followed the quality assurance requirements outlined in the Test Specification/Plan. The descriptions provided in this test report are an accurate account of both the conduct of the work and the data collected. Test plan results are reported. Also reported are any unusual or anomalous occurrences that are different from expected results. The test results and this report have been reviewed and verified.

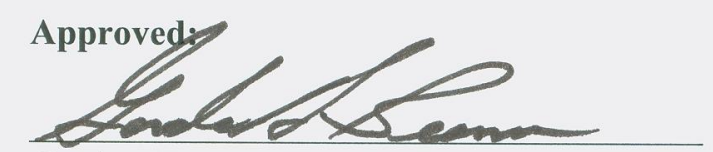

Gordon H. Beeman, Manager WTP R\&T Support Project

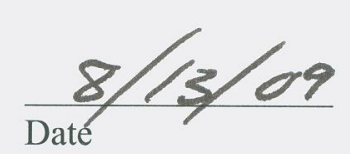




\section{Contents}

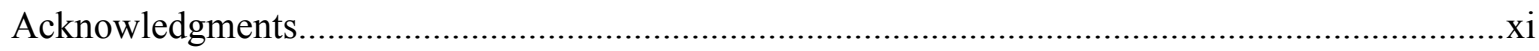

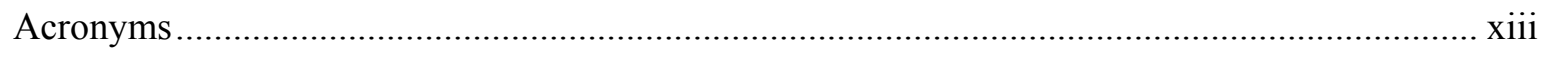

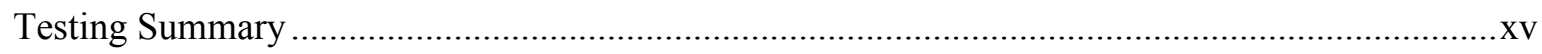

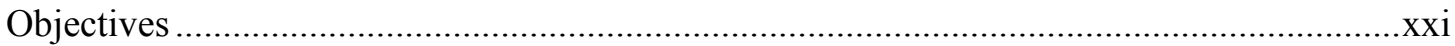

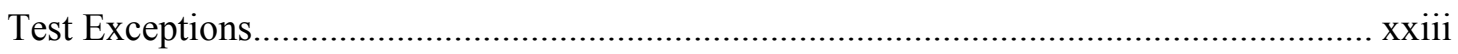

Results and Performance Against Success Criteria ...........................................................xxvi

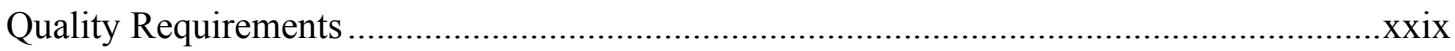

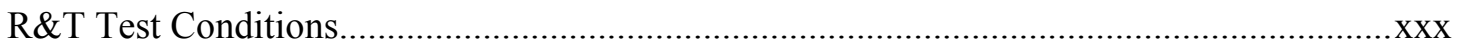

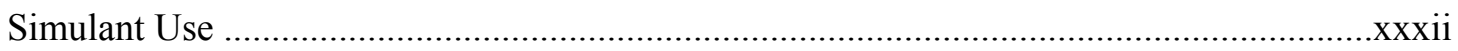

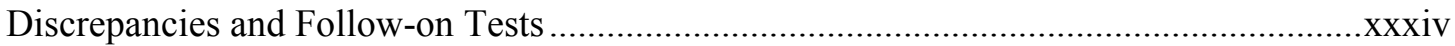

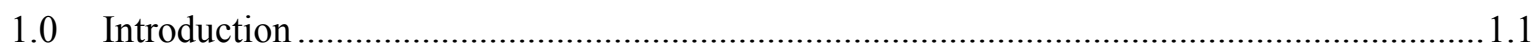

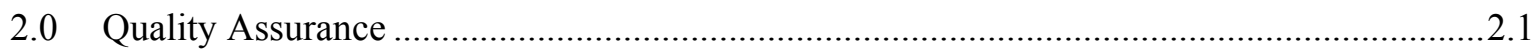

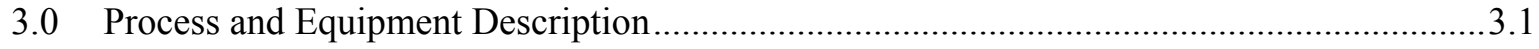

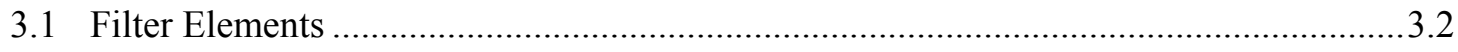

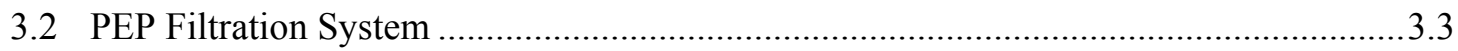

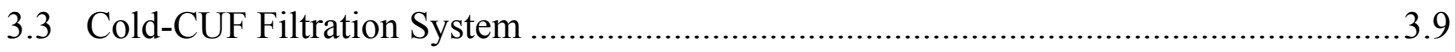

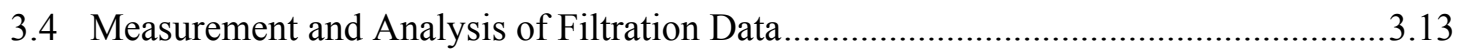

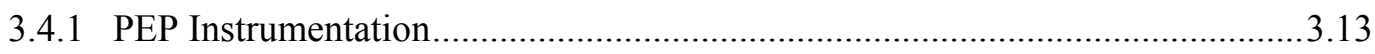

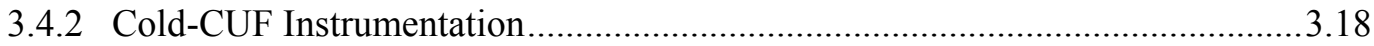

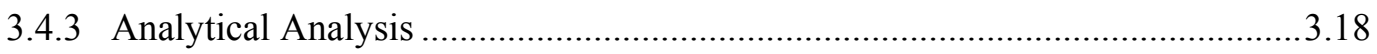

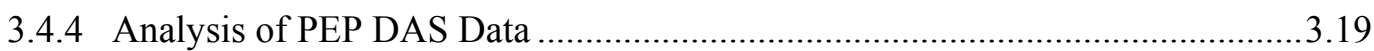

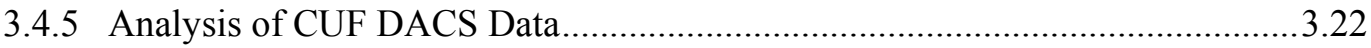

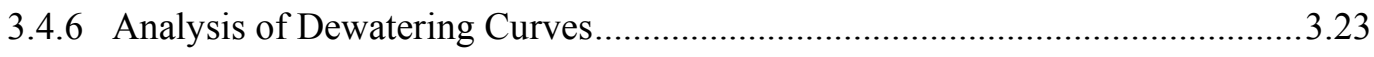

3.4.7 Slurry Solids-to-Filter Surface Area Ratio ...........................................................2.25

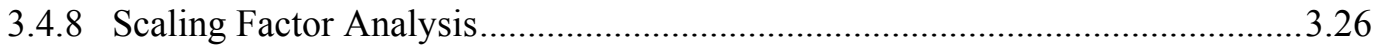

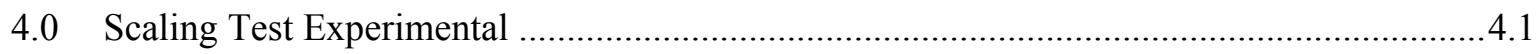

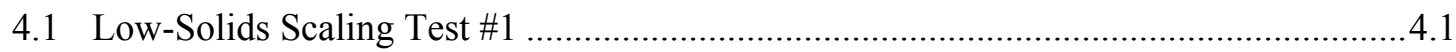

4.1.1 PEP Operations for Low-Solids Scaling Test \#1 ............................................. 4.2

4.1.2 CUF Operations for Low-Solids Scaling Test \#1 .............................................. 4.5

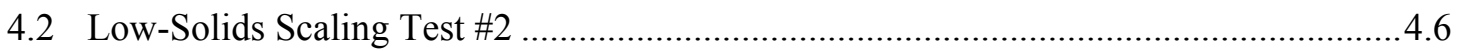

4.2.1 PEP Operations for Low-Solids Scaling Test \#2 …............................................ 4.6

4.2.2 CUF Operations for Low-Solids Scaling Test \#2 ............................................. 4.7

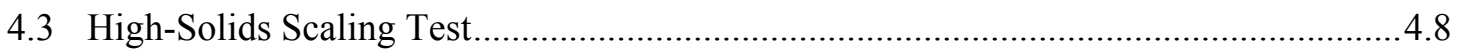

4.3.1 PEP Operations for High-Solids Scaling ....................................................... 4.8

4.3.2 CUF Operations for High-Solids Scaling...................................................... 4.11 


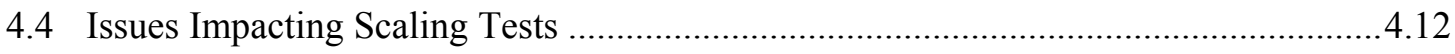

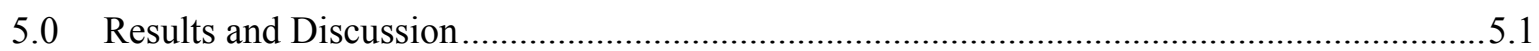

5.1 Test Results for Low-Solids Scaling Test \#1 ….........................................................

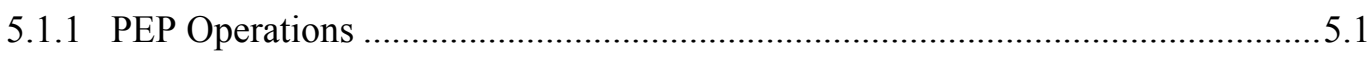

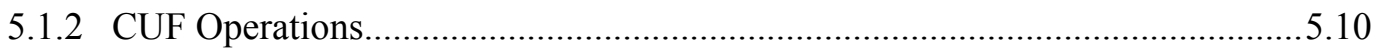

5.1.3 Analysis of Filter Scaling for Low-Solids Test \#1 ….......................................5.15

5.2 Test Results for Low-Solids Scaling Test \#2 ..........................................................5.20

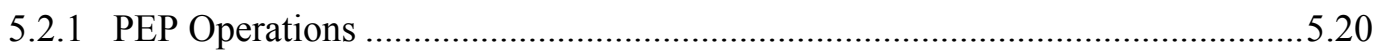

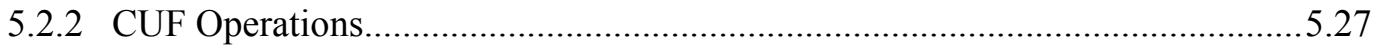

5.2.3 Analysis of Filter Scaling for Low-Solids Test \#2 .........................................5.32

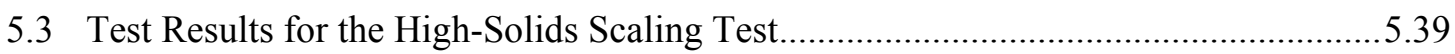

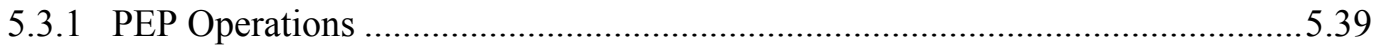

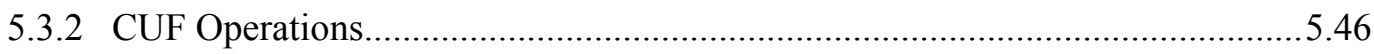

5.3.3 Analysis of Filter Scaling for the High-Solids Test ........................................5.51

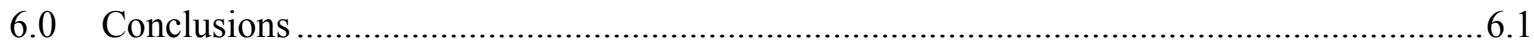

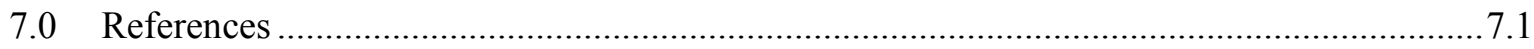




\section{Figures}

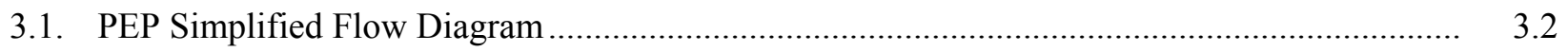

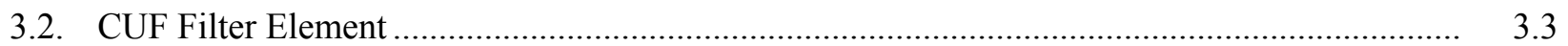

3.3. PEP Filter Bundle Assembly with Key Geometric Parameters Listed (drawing not to scale).... 3.5

3.4. PEP Filter Element Arrangement (axial and side views - drawings not to scale) .................... 3.5

3.5. Simplified Schematic of the Flow Connections for the PEP Permeate Metering and

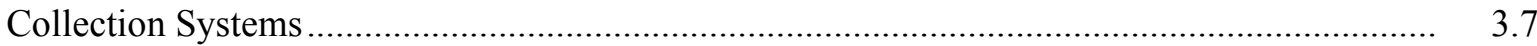

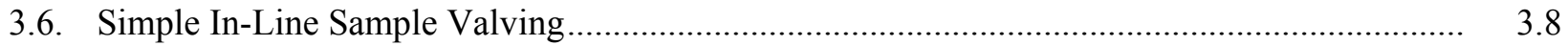

3.7. In-Tank Sample Collection System for Tank T02A Showing the Three Radial Positions at Three Heights and Sampling Flow Loop ............................................................................ 3.9

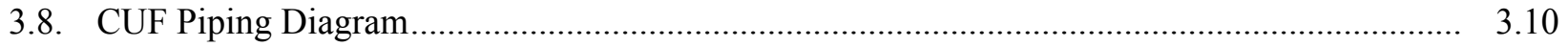

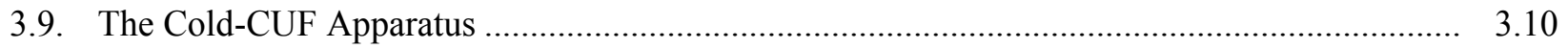

3.10. CUF Filter Assembly Sketch (not to scale) ..................................................................... 3.11

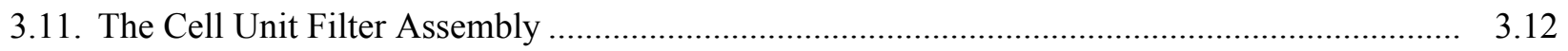

3.12. Diagram of DACS System ........................................................................................ 3.19

3.13. Typical Filter Flux Behavior as a Function of Solids Concentration ........................................ 3.24

5.1. Filter AVs Achieved at PEP During Low-Solids Scaling Test \#1 _........................................ 5.3

5.2. Filter TMPs Achieved at PEP During Low-Solids Scaling Test \#1 ........................................ 5.4

5.3. Filter APDs Observed at PEP During Low-Solids Scaling Test \#1 ....................................... 5.5

5.4. Temperature of Vessel UFP-VSL-T02A During Low-Solids Scaling Test \#1 ........................ 5.5

5.5. Individual Permeate Flux for PEP Filters (corrected for variation in TMP and temperature) During Low-Solids Scaling Test \#1 ................................................................................ 5.7

5.6. Total Permeate Flux for PEP filters (normalized for variation in TMP and temperature) During Low-Solids Scaling Test \#1 ................................................................................ 5.10

5.7. CUF Filter AV During Low-Solids Scaling Test \#1 _...................................................... 5.12

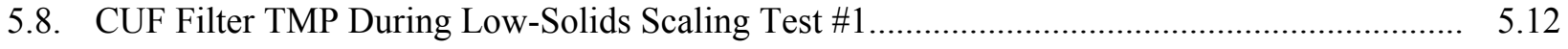

5.9. CUF Filter APD During Low-Solids Scaling Test \#1 ....................................................... 5.13

5.10. CUF Slurry Reservoir Temperature During Low-Solids Scaling Test \#1 ............................... 5.13

5.11. CUF Filter Flux (normalized for TMP and temperature variations) During Low-Solids

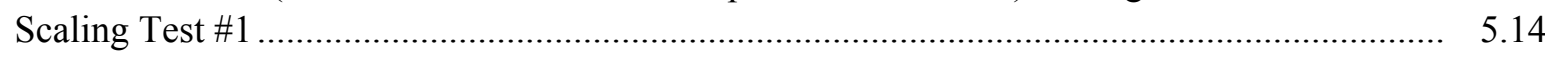

5.12. Comparison of CUF Filter Flux to Individual PEP Filter Bundle Flux During Low-Solids

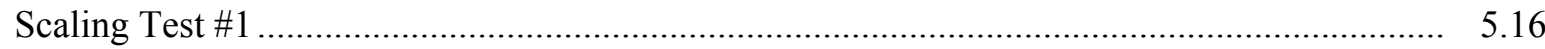

5.13. Comparison of CUF Filter Flux to Total PEP Filter Flux During Low-Solids Scaling Test \#1

5.14. PEP to CUF Scaling Factor all Five PEP Filters as a Function of Time for Low-Solids Scaling Test \#1 


\section{Figures}

5.15. Total PEP to CUF Scaling Factor as a Function of Time for Low-Solids Scaling Test \#1........ 5.19

5.16. Filter AVs Achieved at PEP During Low-Solids Scaling Test \#2 ......................................... 5.22

5.17. Filter TMPs Achieved at PEP During Low-Solids Scaling Test \#2 ...................................... 5.22

5.18. Filter APDs Observed at PEP During Low-Solids Scaling Test \#2 ...................................... 5.23

5.19. Temperature of Vessel UFP-VSL-T02A During Low-Solids Scaling Test \#2 ......................... 5.24

5.20. Individual Permeate Flux for PEP Filters (corrected for TMP and temperature variations) During Low-Solids Scaling Test \#2 ......................................................................... 5.26

5.21. Total Permeate Flux for PEP Filters (normalized for TMP and temperature variations) During Low-Solids Scaling Test \#2 _............................................................................. 5.27

5.22. CUF Filter AV During Low-Solids Scaling Test \#2 _......................................................... 5.29

5.23. CUF Filter TMP During Low-Solids Scaling Test \#2 _.......................................................... 5.29

5.24. CUF Filter APD During Low-Solids Scaling Test \#2 ........................................................ 5.30

5.25. CUF Slurry Reservoir Temperature During Low-Solids Scaling Test \#2 ................................ 5.30

5.26. CUF Filter Flux (corrected for TMP and temperature variations) During Low-Solids

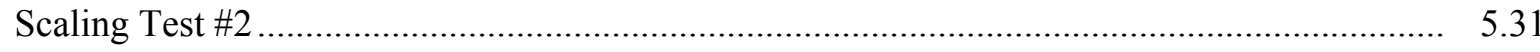

5.27. Comparison of CUF Filter Flux to Individual PEP Filter Bundle Flux During Low-Solids Scaling Test \#2. All filter fluxes have been corrected for TMP and temperature variations ..... 5.33

5.28. Comparison of CUF Filter Flux to Total PEP Filter Flux During Low-Solids Scaling Test \#2 . 5.34

5.29. PEP to CUF Scaling Factor all Five PEP Filters as a Function of Time for Low-Solids

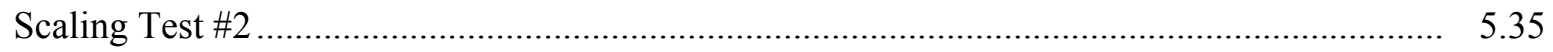

5.30. Total PEP to CUF Scaling Factor as a Function of Time for Low-Solids Scaling Test \#2........ 5.36

5.31. Comparison of Total PEP Filter Flux for Low-Solids Scaling Tests \#1 and \#2 ….................. 5.38

5.32. Comparison of Total CUF Filter Flux for Low-Solids Scaling Tests \#1 and \#2 ..................... 5.38

5.33. Slurry UDS Concentrations Tested During the PEP High-Solids Scaling Test ....................... 5.40

5.34. Filter AVs Achieved During the PEP High-Solids Scaling Test ........................................... 5.41

5.35. Filter TMP for Filter Bundle 1 During the PEP High-Solids Scaling Test .............................. 5.42

5.36. Filter APD Across Filter Bundle 1 During the PEP High-Solids Scaling Test ......................... 5.44

5.37. Temperatures Measured in Vessel UFP-VSL-T02A and in the Filtration Loop During the PEP High-Solids Scaling Test .................................................................................. 5.44

5.38. Dewatering Curve for the High-Solids Simulant Measured During the PEP High-Solids

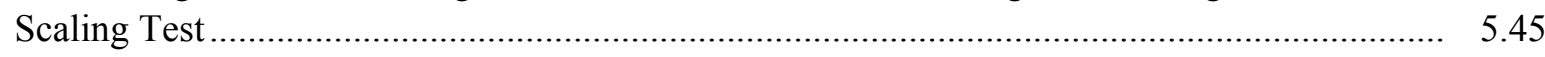

5.39. Slurry UDS Concentrations Tested During the Initial and Repeat and CUF High-Solids

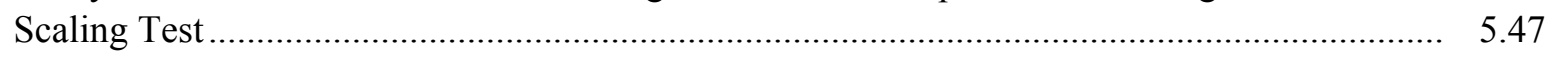

5.40. Filter AVs Achieved During the Initial and Repeat CUF High-Solids Scaling Test ................ 5.48

5.41. Filter TMP Achieved During the Initial and repeat CUF High-Solids Scaling Test.................. 5.48 


\section{Figures}

5.42. Filter APD Across the CUF Filter During the Initial and Repeat CUF High-Solids Scaling

5.43. Temperature of the CUF Slurry Reservoir During the Initial and Repeat CUF High-Solids Scaling Test.

5.44. Dewatering Curves for the High-Solids Simulant Measured During the Initial and Repeat CUF High-Solids Scaling Tests

5.45. Comparison of the PEP and CUF Dewatering Curves.

5.46. Analysis of the PEP Dewatering Curve Measured During the PEP High-Solids Scaling Test

5.47. Analysis of the Initial Dewatering Curve Measured During the CUF High-Solids Scaling Test.

5.48. Analysis of the Repeat Dewatering Curve Measured During the CUF High-Solids Scaling Test. 


\section{Tables}

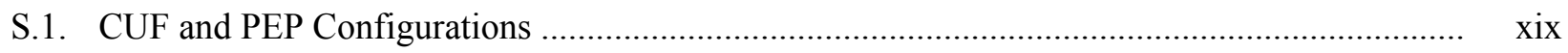

S.2. Results for Low-Solids Scaling Tests ......................................................................... $\mathrm{xx}$

S.3. Results for High-Solids Scaling Test .............................................................................. $\quad$ xxii

S.4. Summary of Test Objectives and Results...................................................................... xxiv

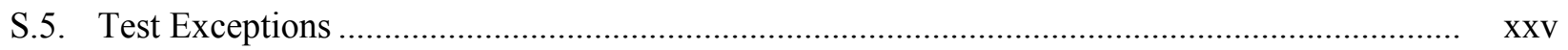

S.6. Deviations from Test Specification ....................................................................................... xxviii

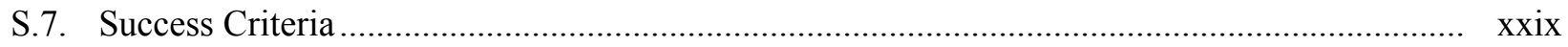

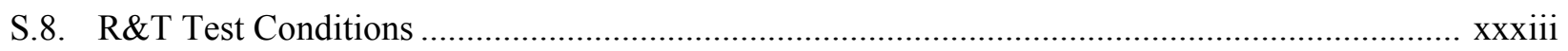

3.1. Specifications of the Five PEP Cross-Flow Filtration Bundles............................................. 3.6

3.2. Permeate Metering and Pulse Pot Configurations for PEP ................................................ 3.6

3.3. PEP Instrumentation Used to Assess Slurry Flowrates Through the Filtration Circulation

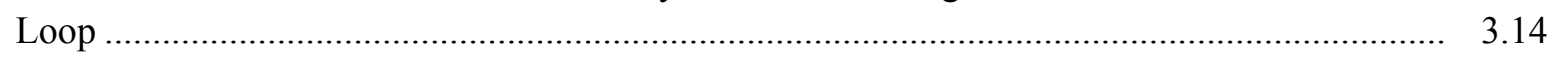

3.4. PEP Filter Bundle Pressure Sensing Instrumentation ..................................................... 3.15

3.5. PEP Coriolis Flow Meters for Permeate Production Rate Measurement .................................. 3.15

3.6. PEP Test Operational Procedure Summary ........................................................................ 3.17

3.7. CUF Filtration System Measurement Instrumentation...................................................... 3.18

4.1. Target Operational Conditions for Low-Solids Scaling Test \#1 _............................................ 4.3

4.2. A List of Slurry Sampling for Low-Solids Scaling Test \#1 ................................................

4.3. Target Operational Conditions for Low-Solids Scaling Test \#2 _.......................................... 4.7

4.4. A List of Slurry Sampling for Low-Solids Scaling Test \#2 ................................................ 4.7

4.5. Target Operational Conditions for High-Solids Scaling Test ................................................ 4.10

4.6. A List of Slurry Sampling for High-Solids Scaling Test .....................................................

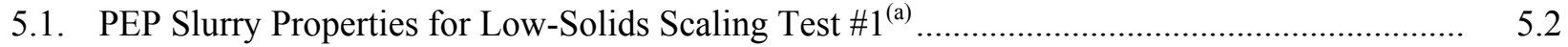

5.2. Operation Conditions Achieved for Tank T02A PJM and Sparging Systems During Low-

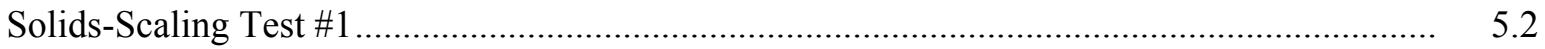

5.3. Test Conditions and Operational Parameters for CUF Low-Solids Scaling Test \#1 ................ 5.11

5.4. Average PEP to CUF Scaling Factors for Filter Flux as a Function of Filter Bundle and as a Function of Process Operation ..................................................................................... 5.20

5.5. PEP Slurry Properties for Low-Solids Scaling Test \#2 .................................................... 5.21

5.6. Operation Conditions Achieved for Tank T02A PJM and Sparging Systems During Low-

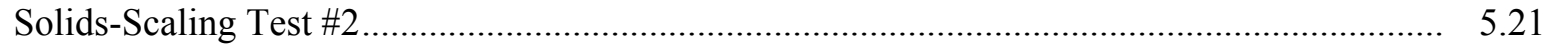

5.7. Test Conditions and Operational Parameters for CUF Low-Solids Scaling Test \#2 ................ 5.28

5.8. Average PEP to CUF Scaling Factors for Filter Flux as a Function of Filter and as a Function of Process Operation ........................................................................................... 5.36

5.9. PEP Slurry Properties for the High-Solids Scaling Test ................................................ 5.40 


\section{Tables}

5.10. Operation Conditions Achieved for Tank T02A PJM and Sparging Systems During the High-Solids-Scaling Test ............................................................................................ 5.40

5.11. Operational Parameters for the High-Solids Dewatering Tests............................................ 5.46

5.12. Summary of PEP and CUF High-Solids Dewatering Curve Parameters ................................ 5.54

5.13. Summary of PEP to CUF Scaling Factors for High-Solids Dewatering Operations.................. 5.55

6.1. Differences Between CUF and PEP .........................................................................................

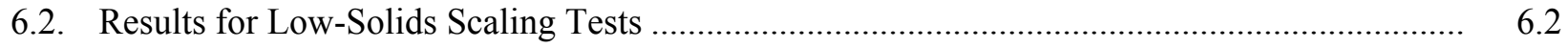

6.3. Results for High-Solids Scaling Tests.................................................................................. 



\section{Acknowledgments}

The authors would like to thank Wayne Cosby and Michael Bates for their extensive editorial support, Dave MacPherson and Kirsten Meier for Quality Assurance support and reviews, and Reid Peterson and Rick Shimskey for their careful and thorough technical reviews of this report. In addition to these PNNL staff, this report was also supported by many dedicated staff involved in laboratory experiments, sampling, data acquisition, data processing, data-quality confirmation, technical reviews, and data analysis.

$\begin{array}{lll}\text { JM Alzheimer } & \text { JM Cuta } & \text { RL Russell } \\ \text { C Arimescu } & \text { AM Casella } & \text { RD Scheele } \\ \text { DD Bachand } & \text { MS Fountain } & \text { PP Schonewill } \\ \text { EBK Baer } & \text { JGH Geeting } & \text { GJ Sevigny } \\ \text { S Barnes } & \text { EC Golovich } & \text { SV Shoemaker } \\ \text { AW Baumann } & \text { CE Guzman-Leong } & \text { DM Smith } \\ \text { GH Beeman } & \text { RE Hohimer } & \text { PS Sundar } \\ \text { DL Blanchard Jr } & \text { WL Kuhn } & \text { JJ Toth } \\ \text { JC Bower } & \text { WE Lawrence } & \text { JC Tucker } \\ \text { OP Bredt } & \text { S Lehrman } & \text { MW Urie } \\ \text { GN Brown } & \text { D Neiner } & \text { BE Wells } \\ \text { CA Burns } & \text { RP Pires } & \text { JK Young } \\ \text { SA Bryan } & \text { DE Rinehart } & \text { ST Yokuda }\end{array}$

These individuals and the rest of the operations and sampling crew are especially recognized for supporting the Pretreatment Engineering Platform Project by working unpredictable, long, and off-hour shifts for the better part of a year. The tests and reports could not have gotten this far without their extraordinary effort. 



\section{Acronyms}

APD axial pressure drop

APEL Applied Process and Engineering Laboratory

ASME American Society of Mechanical Engineers

AV

axial velocity

BNI Bechtel National Inc.

CD Coriolis densitometer

CS centrifuged solids

CUF Cells Unit Filter

DACS data-acquisition collection system

DAS data acquisition system

DOE U.S. Department of Energy

DS dissolved solids

EFRT External Flowsheet Review Team

GPM gallons per minute

HLW high-level waste

LAW low-activity waste

PEP

Pretreatment Engineering Platform

PJM pulse jet mixer

PNNL Pacific Northwest National Laboratory

PSD particle-size distribution

psig pounds per square inch gauge

psid pounds per square inch differential

PTF Pretreatment Facility

QA quality assurance

QAP Quality Assurance Plan

QARD Quality Assurance Requirements and Descriptions

RPP River Protection Project

R\&T research and technology

RTD resistance temperature detector

SBMS Standards-Based Management System

SWRI Southwest Research Institute

TMP transmembrane pressure

TS total solids

UDS undissolved solids

UFP ultrafiltration process

WTP Hanford Tank Waste Treatment and Immobilization Plant 



\section{Testing Summary}

Pacific Northwest National Laboratory (PNNL) has been tasked by Bechtel National Inc. (BNI) on the River Protection Project-Hanford Tank Waste Treatment and Immobilization Plant (RPP-WTP) project to perform research and development activities to resolve technical issues identified for the Pretreatment Facility (PTF). The Pretreatment Engineering Platform (PEP) was designed, constructed, and operated as part of a plan to respond to issue M12, "Undemonstrated Leaching Processes" of the External Flowsheet Review Team (EFRT) issue response plan. ${ }^{(a)}$ The PEP is a $1 / 4.5$-scale test platform designed to simulate the WTP pretreatment caustic leaching, oxidative leaching, ultrafiltration solids concentration, and slurry washing processes. The PEP replicates the WTP leaching processes using prototypic equipment and control strategies. The PEP also includes non-prototypic ancillary equipment to support the core processing.

Two operating scenarios are currently being evaluated for the ultrafiltration process (UFP) and leaching operations. The first scenario has caustic leaching performed in the UFP-2 ultrafiltration feed vessels (i.e., vessel UFP-VSL-T02A in the PEP and vessels UFP-VSL-00002A and B in the WTP PTF). The second scenario has caustic leaching conducted in the UFP-1 ultrafiltration feed preparation vessels (i.e., vessels UFP-VSL-T01A and B in the PEP; vessels UFP-VSL-00001A and B in the WTP PTF).

In both scenarios, 19-M sodium hydroxide solution ( $\mathrm{NaOH}$, caustic) is added to the waste slurry in the vessels to leach solid aluminum compounds (e.g., gibbsite, boehmite). Caustic addition is followed by a heating step that uses direct injection of steam to accelerate the leach process. Following the caustic leach, the vessel contents are cooled using vessel cooling jackets and/or external heat exchangers. The main difference between the two scenarios is that for leaching in UFP-1, the $19-\mathrm{M} \mathrm{NaOH}$ is added to unconcentrated waste slurry (3 to $8 \mathrm{wt} \%$ solids), while for leaching in UFP-2, the slurry is concentrated to nominally $20 \mathrm{wt} \%$ solids using cross-flow ultrafiltration before the addition of caustic.

The PEP testing program was conducted under Test Plan TP-RPP-WTP-506 ${ }^{(\mathrm{b})}$ using a waste simulant that was developed in response to Task 5 from the M-12 External Flowsheet Review Team (EFRT) issue response plan. ${ }^{(a)}$ The testing included the following tests with simulated Hanford tank waste:

- Shakedown/Functional Testing: Tested process operations (e.g., slurry transfers, steam heating of the vessels, and the accumulation of condensate, filter backpulsing and flushing), process controls (e.g., transmembrane pressure (TMP) and axial flow velocity in the filter loop), and certain test functions (e.g., in-line slurry sampling accuracy and precision).

- Integrated Test A: Demonstrated integrated processing when caustic leaching $\left(98^{\circ} \mathrm{C}\right)$ is performed in UFP-VSL-00001A/B with the Cr simulant component added after the post-caustic leach washing step.

(a) SM Barnes, and R Voke. 2006. "Issue Response Plan for Implementation of External Flowsheet Review Team (EFRT) Recommendations - M12: Undemonstrated Leaching Process." 24590-WTP-PL-ENG-06-0024 Rev. 0.

(b) GB Josephson, OP Bredt, JK Young, and DE Kurath. 2009. Test Plan for Pretreatment Engineering Platform (PEP) Testing (Phase I). TP-RPP-WTP-506, Rev. 0.4, Pacific Northwest National Laboratory, Richland, Washington. 
- Integrated Test B: Demonstrated integrated processing when the caustic leaching $\left(98^{\circ} \mathrm{C}\right)$ is performed in UFP-VSL-00002A with the Cr simulant component added after the post-caustic leach washing step.

- Integrated Test D: Demonstrated integrated processing when the caustic leaching is performed at a lower temperature $\left(85^{\circ} \mathrm{C}\right)$ in UFP-VSL-00002A and with the $\mathrm{Cr}$ simulant component added to the initial batch of simulant.

Integrated Test $\mathrm{C}$ was deleted from the scope of the testing (ICN-TP-RPP-WTP-506_R0.2).

The work described in this report presents filter flux results obtained at two different scales based on tests performed with a Hanford tank waste simulant. The tests were made at the laboratory-bench scale on a cold (i.e., designated for non-radioactive simulant test materials) Cells Unit Filter (CUF) and in the Pretreatment Engineering Platform (PEP). PEP has up to 276 times the filter area available in CUF. One set of tests was conducted with the simulant feed (low solids) and one test was conducted at a relatively high solids concentration. The results of these tests are compared to support the development of a scale factor for use in the WTP by determining issues in PEP to CUF scaling.

To facilitate the analysis of system scaling, CUF and PEP operations are designed to be equivalent. Both systems use similar filter elements (Mott sintered stainless steel filter tubes of 0.5 -inch inner diameter) taken from the same manufacturer's lot. Both test configurations are similar - a filtration loop is fed from a slurry reservoir/tank with the filtration loop being composed of a slurry pumping system, filtration area, permeate collection and metering systems, heat exchanger (to remove mechanical heat), and filtration-loop backpressure valve. Despite these similarities, many operational/configurational differences exist that could yield differences in PEP and CUF scaling. As expected, the most dominant difference is size - as stated previously, PEP has up to 276 times the filter area available in CUF. Other key differences that could limit scaling from CUF to PEP are summarized in Table S.1. 
Table S.1. CUF and PEP Configurations

\begin{tabular}{|c|c|c|}
\hline Item & CUF Configuration & PEP Configuration \\
\hline Filtration area & $\begin{array}{l}\text { CUF testing employs a single } 2 \text {-ft-long } \\
\text { filter element comprising a total filtration } \\
\text { area of } 0.262 \mathrm{ft}^{2} .\end{array}$ & $\begin{array}{l}\text { PEP testing employs multiple filter elements } \\
\text { consisting of a mixture of } 8 \text {-ft-long and } \\
10 \text {-ft-long filter elements. Elements are } \\
\text { fixed in bundles containing } 12 \text { filters each. } \\
\text { There are five filter bundles total, } \\
\text { comprising a total filtration area of up to } \\
72.3 \mathrm{ft}^{2} \text { (276 times that of CUF). }\end{array}$ \\
\hline $\begin{array}{l}\text { Process } \\
\text { configuration }\end{array}$ & $\begin{array}{l}\text { (1) Pumping system, (2) heat exchanger, } \\
\text { (3) filter element, (4) backpressure valve, } \\
\text { (5) slurry reservoir. } \\
\text { Heat exchange precedes the filtration area. }\end{array}$ & $\begin{array}{l}\text { (1) Pumping system, (2) filtration bundles, } \\
\text { (3) heat exchanger, (4) backpressure valve, } \\
\text { (5) slurry reservoir. } \\
\text { Heat exchange follows the filtration area. }\end{array}$ \\
\hline Pumps & A single rotary lobe slurry pump. & $\begin{array}{l}\text { Two centrifugal slurry pumps operated in } \\
\text { series. }\end{array}$ \\
\hline $\begin{array}{l}\text { Slurry reservoir } \\
\text { mixing system }\end{array}$ & $\begin{array}{l}\text { A single overhead agitator mixes the } \\
\text { slurry reservoir. Additional mixing is } \\
\text { provided by the slurry return from the } \\
\text { filtration loop. }\end{array}$ & $\begin{array}{l}\text { Slurry tank mixing is provided by pulse jet } \\
\text { mixers (PJMs) and air spargers. Additional } \\
\text { mixing is provided by the slurry return from } \\
\text { the filtration loop. }\end{array}$ \\
\hline Filter history & $\begin{array}{l}\text { The filter employed for CUF testing has } \\
\text { been used extensively in simulant } \\
\text { development and testing activities } \\
\text { throughout calendar year } 2008 \text {. }\end{array}$ & $\begin{array}{l}\text { The filter bundles employed for PEP testing } \\
\text { are relatively new and have not been used } \\
\text { extensively. Previous testing is limited to } \\
\text { primarily water functional testing. Contact } \\
\text { with waste simulant slurry is limited. }\end{array}$ \\
\hline
\end{tabular}

Three separate scaling tests were performed to assess scaling effects that exist between PEP engineering-scale filtration operations and CUF bench-scale filtration operations. These tests were:

- Low-Solids Scaling Test \#1: A 36-hr low-solids concentration continuous/backpulsed recycle filtration operation

- Low-Solids Scaling Test \#2: A repeat of the 36-hr low-solids concentration continuous/backpulsed recycle filtration operation

- High-Solids Scaling Test: A high-solids dewatering operation.

It should be noted that both low-solids tests were also intended to "condition" (i.e., extensively expose to and contact) the filter against the simulant slurry solids employed for subsequent tests. For each test run at the PEP, a parallel test was run on the CUF filtration system located at the Applied Process and Engineering Laboratory (APEL). These tests allow assessment of the PEP to CUF scaling factor for continuous and backpulsed recycle operations and for dewatering operations approaching the slurry gel point. Parallel PEP and CUF tests were performed at similar slurry solids-to-filter surface area ratios (and using filter elements of similar manufacture). The high-solids scaling test was performed at a slurry solids-to-filter surface area ratio prototypic of WTP operations; however, both low-solids scaling tests 
were performed at ratios slightly lower than that anticipated for use in WTP. ${ }^{(a)}$ With respect to the latter, previous scaling studies in WTP-RPT-168, Rev. 0, suggest that this difference is not expected to impact filtration scaling substantially (Daniel et al. 2009). As such, the low-solids scaling test results likely represent the lower bound of the solids to filter-area-ratio expected in the PTF.

The low-solids scaling tests considered the performance of PEP filtration (as measured through filter flux corrected to standard temperatures and TMPs) against that observed on the CUF test system. The scaling factor was defined as the ratio of PEP filter flux to CUF filter flux. The low-solids scaling tests indicate that for similarly conditioned filters, the CUF flux is comparable to, but slightly under predicts, the total (area averaged) flux obtained at PEP. The final filter scaling factors based on total (areaaveraged) PEP flux for low-solids tests $\# 1$ and $\# 2$ were both $1.1 \pm 0.1$. To provide a conservative estimate for process scaling, a scaling factor of 1.0 is recommended for scaling low-solids filtration operations. A summary of results for the low-solids scaling tests (and key operational parameters) is included in Table S.2.

Table S.2. Results for Low-Solids Scaling Tests

\begin{tabular}{|l|c|c|c|c|}
\hline Item & CUF & PEP & CUF & PEP \\
\hline Test Description & Low-Solids Test \#1 & Low-Solids Test $\# 2$ \\
\hline Target axial velocity (AV) (ft/s) & 15.0 & $15.0 \pm 1.4$ & 15.0 & $15.0 \pm 1.4$ \\
\hline Actual Average AV (ft/s) & $14.9 \pm 0.7$ & 14.8 & $15.0 \pm 0.6$ & 14.8 \\
\hline Target TMP (psid) & 40 & $40 \pm 4$ & 40 & $40 \pm 4$ \\
\hline Actual TMP (psid) & $40.2 \pm 0.8$ & 39.8 & $40.2 \pm 0.4$ & 39.9 \\
\hline Filtration Area (ft ${ }^{2}$ ) & 0.262 & 72.3 & 0.262 & 72.3 \\
\hline Solids-To-Filter Area Ratio (kg/ $\mathrm{ft}^{2}$ ) & 1.5 & 1.1 & 1.4 & 1.4 \\
\hline Flux Scaling Factor Range (S) & \multicolumn{2}{|c|}{1.1 to 1.4} & \multicolumn{2}{c|}{1.1 to 1.2} \\
\hline Recommended Scaling Factor & \multicolumn{2}{|c|}{$\mathbf{1 . 0}$} & \multicolumn{2}{c}{} \\
\hline
\end{tabular}

With regard to the "alternate" goal of filter conditioning, which was to minimize history differences in CUF and PEP by exposing the filter elements to a similar slurry, the conditioning of the filters appears to have been successful from a total (area-averaged) flux standpoint. Specifically, PEP and CUF flux differ substantially (up to $40 \%$ ) during the initial run-in period of 12-hrs. In both low-solids scaling tests, a convergence of total filter flux is observed during the second 12-hr period of backpulsed operations, yielding similar CUF and PEP fluxes during the final 12-hrs of operation. Overall, exposure of the filter membrane to slurry solids appears to have reduced potential impacts from differing CUF and PEP histories. However, it should be noted that history effects are difficult to distinguish from potential scaling effects. Additionally, frequent backpulsing of the filter appears to be the best driver of filter conditioning. It is speculated that frequent disruption of the protective cake layer allows significant exposure and contact between the filter membrane and slurry solids.

It should be noted that this low-solids operations scaling factor estimate is subject to limitations associated with the test. These limitations derive from the following:

(a) For the purposes of slurry solids-to-filter surface area ratios, an expected range of 1.7 to $16 \mathrm{~kg}$ solids per square foot of effective filter area has been estimated. This estimate is based on the parameters outlined in Sections 2.3.4.1.1 and 2.3.4.1.2 in 24590-WTP-RPT-PT-02-005, Rev. 4. 
- Divergence of filter flux from individual PEP filter bundles. Both the first and second low-solids tests examined three separate test segments: 1) an initial 12-hr period of continuous (non-backpulsed) recycle filtration, 2) a 12-hr period of backpulsed operations with 24 total backpulses at 30 minute intervals, and 3) a final 12-hr period of continuous (non-backpulsed) recycle filtration. Recycle filtration in all segments employed all five PEP filter bundles. During the first 12-hr segment, filter flux for all five bundles was comparable. However, during the second 12-hr segment, backpulsed operations caused a divergence in the filter flux across each filter. Flux from the upstream bundles was relatively constant throughout backpulsing. In contrast, the downstream filters showed irrecoverable flux loss throughout backpulsing. It is speculated that flux loss on the downstream filters is caused by irreversible depth fouling of the porous filter element during the interim period between cake disruption and cake formation after each backpulse operation. However, this mechanism does not explain why the downstream filters are more susceptible to irreversible fouling. Regardless, the speculated preferential fouling of downstream filters (and the relative immunity of upstream filters with respect to fouling) was observed in both low-solids tests and appears reproducible. The difference in filter flux caused by divergence during backpulsed operations persisted into the final 12-hr test segment. At the end of testing, the difference in flux across the filter bundles is still significant - upstream filter flux is 50 to $100 \%$ higher than downstream filter flux.

CUF filter flux appears to fall between the two flux extremes observed in individual PEP filter bundles. As a result, PEP to CUF scaling factors based on individual PEP filter fluxes range from $\sim 0.7$ up to $\sim 1.6$. This indicates that CUF filter flux provides an inexact representation of the flux performance of individual PEP filter bundles (for the low-solids scaling tests). That being stated, the difference in CUF and individual PEP bundle performance is not great. CUF provides an order of magnitude approximation of PEP filter bundle flux and an approximate representation of the flux time dependency. Additionally, when PEP filter flux is considered on a total (i.e., area-averaged) flux basis, it provides an excellent representation PEP performance (with scaling factors close to 1.0 for conditioned filters). Thus, the bench-scale CUF provides an accurate measure of PEP filter flux magnitude and dynamics when the flux across all filters was considered for conditioned filters. The test results for the low-solids scaling test also indicate that CUF filter flux provides a conservatively low estimate of flux for unconditioned filters.

While CUF appears to provide an accurate measure of PEP filter performance, the underlying concern is that flux divergence observed during backpulsing was not expected and is currently not understood. Further study of the mechanisms causing PEP flux divergence is recommended to allow better assessment of their potential impacts on scaling analyses.

- Differences in the state of PEP and CUF initial filter conditioning. The recommended low-solids scaling factor of 1.0 is based on the assumption of similarly conditioned filters. Application to unconditioned filters may require scaling factors different than one. In the low-solids tests presented in the current report, the CUF significantly under predicted the PEP flux during the first 12-hr test segment of the low-solids tests (where CUF and PEP filters are relatively unconditioned by the simulant slurry). The scaling factors associated with the initial 12-hr test segment were $1.4 \pm 0.2$ and $1.2 \pm 0.1$ for first and second low-solids scaling tests, respectively. Filter conditioning reduced this flux discrepancy - at the end of testing, the scaling factors were both $1.1 \pm 0.1$. In short, the best agreement between CUF and PEP total filter flux for the low-solids scaling tests is achieved only after the filters have been conditioned (i.e., fouled) against a similar waste simulant. To enable better 
scaling and comparison (especially for unconditioned filters), an evaluation of the effects of nitric and oxalic acid cleaning on the performance of the filter elements is recommended.

- Insufficient process test time to achieve filtration steady-state. For both low-solids scaling tests performed on the PEP and CUF filtration systems, the 12-hr test segments were insufficient to reach a process steady state (or even to assess the existence/value of a steady state flux). This limitation impacted both CUF and PEP filtration systems, and as such, all filtration results discussed in this report are subject to further time-dependent decay. The lack of a filtration steady state (and continued decline of filter flux throughout the test) does not appear to impact agreement (and subsequent scaling factor analyses) of total PEP and CUF filter fluxes - the scaling factors observed for conditioned filters in the low-solids scaling tests showed little time-dependence and were close to 1.0. However, continued flux decay throughout the test introduces uncertainty with respect to PEP and CUF scaling over time frames longer than those tested. An evaluation of long-term (i.e., much greater than $36 \mathrm{hrs)}$ filter flux dynamics is recommended to assess their potential impacts on scaling of filtration performance.

High-solids scaling factor analysis considered scaling in terms of the parameters characterizing filtration dewatering performance at concentrations approaching the limiting gel concentration. These parameters are 1) the dewatering mass transfer coefficient $(k)$ and 2$)$ the slurry limiting gel concentration $\left(C_{g}\right)$. Two separate scaling factors were defined - the first is the ratio of PEP $k$ to CUF $k$, and the second is the ratio of PEP $C_{g}$ to CUF $C_{g}$.

Analysis of PEP and CUF high-solids dewatering curves indicates scaling factors of $0.97 \pm 0.03$ and $0.96 \pm 0.05$ for both $k$ and $C_{g}$, respectively. These results indicate that the high-solids filtration performance CUF and PEP are indistinguishable from one another. Based on the best information currently available, the scaling factor for high-solids dewatering operations appears to be one. That is, CUF appears to provide an accurate indication of PEP filter flux performance during high-solids dewatering operations approaching the gel point. A summary of results for the high-solids scaling test (and key operational parameters) is included in Table S.3.

Table S.3. Results for High-Solids Scaling Test

\begin{tabular}{|l|c|c|}
\hline Item & CUF & PEP \\
\hline Test Description & \multicolumn{2}{|c|}{ High Solids Test } \\
\hline Target AV (ft/s) & 15.0 & $15.0 \pm 1.4$ \\
\hline Actual Average AV $(\mathrm{ft} / \mathrm{s})$ & $15.0 \pm 0.1$ & 14.7 \\
\hline Target TMP $(\mathrm{psid})$ & 40 & $40 \pm 4$ \\
\hline Actual TMP $(\mathrm{psid})$ & $41 \pm 1$ & 39.8 \\
\hline Filtration Area $\left(\mathrm{ft}^{2}\right)$ & 0.262 & 15.7 \\
\hline Solids-To-Filter Area Ratio $\left(\mathrm{kg} / \mathrm{ft}^{2}\right)$ & 14.5 & 13.9 \\
\hline Dewatering Mass Transfer Coefficient $\left(\mathrm{GPM} / \mathrm{ft}^{2}\right)$ & $-0.112 \pm 0.001$ & $-0.108 \pm 0.003$ \\
\hline Limiting Gel Concentration $(\mathrm{wt} \%)$ & $34.3 \pm 1.9$ & $35.7 \pm 0.5$ \\
\hline Mass Transfer Scaling Factor $\left(\mathrm{S}_{\mathrm{k}}\right)$ & \multicolumn{2}{|c|}{$0.97 \pm 0.03$} \\
\hline Limit Gel Concentration Scaling Factor $\left(\mathrm{S}_{\mathrm{g}}\right)$ & \multicolumn{2}{|c|}{$0.96 \pm 0.05$} \\
\hline Recommended Scaling Factor & \multicolumn{2}{|c|}{$\mathbf{1 . 0}$} \\
\hline
\end{tabular}


The focus of this report is the analysis of the CUF and PEP test results with the goal of establishing the relationships between these two scaled systems. However, the most direct application of the scale-up analysis is in the WTP G-2 process performance model where the filter performance data from the CUF is to be used to predict PTF performance. For G-2, the scale-up of CUF to PTF is assumed to be a combination of the scale-up of CUF to PEP and the scale-up of PEP to PTF. Since there are no experimental data from the PTF at this point, the PEP to PTF scale-up is based on prototypic design and operation.

The PEP ultrafiltration system has dimensionally prototypic filters and feed vessels, and functionally prototypic pumps, instrumentation, and controls (24590-PTF-3YD-UFP-00002). ${ }^{\text {(a) }}$ The PEP has five filter bundles, like the PTF. Also, PEP ultrafiltration test conditions and operational parameters were chosen to be prototypic of the PTF (24590-WTP-RPT-PET-07-002 ${ }^{\text {(b) }}$ and TP-RPP-WTP-506 ${ }^{(\mathrm{c})}$ ). Given the same simulant, operating conditions, and comparable filter histories, PTF filter performance (i.e., permeate flux, response to backpulsing, cake formation, depth-fouling, filter entrance effect, axial pressure drop, etc.) should be the same as the observed PEP filter performance.

\section{Objectives}

Table S.4 summarizes the objectives along with a discussion of how the objectives were met. The objectives for the entire PEP testing program are provided with discussion limited to those objectives met by the scope of this report. Objectives not met by the scope of this report are shaded in gray.

(a) B Stiver. 2007. Functional Requirements for Pretreatment Engineering Platform (PEP). 24590-PTF-3YDUFP-00002 Rev. 1, Bechtel National Incorporated, Richland, Washington.

(b) S Lehrman. 2008. Pretreatment Engineering Platform (PEP) Phase I Testing Process Description. 24590WTP-RPT-PET-07-002, Rev. 1, Bechtel National Incorporated, Richland, Washington.

(c) GB Josephson, OP Bredt, JK Young, and DE Kurath. 2009. Test Plan for Pretreatment Engineering Platform (PEP) Testing (Phase I). TP-RPP-WTP-506, Rev. 0.4, Pacific Northwest National Laboratory, Richland, Washington. 
Table S.4. Summary of Test Objectives and Results

\begin{tabular}{|c|c|c|}
\hline Test Objective & $\begin{array}{l}\text { Objective } \\
\text { Met? }\end{array}$ & Discussion \\
\hline $\begin{array}{l}\text { Caustic leach process: Compare } \\
\text { engineering- and laboratory-scale } \\
\text { results to determine impact of } \\
\text { scale-up }\end{array}$ & NA & $\begin{array}{l}\text { Results to meet this objective are discussed in report WTP-RPT-186 and } \\
\text { WTP-RPT-197. }\end{array}$ \\
\hline $\begin{array}{l}\text { Oxidative leach process: Compare } \\
\text { engineering- and laboratory-scale } \\
\text { results to determine impact of } \\
\text { scale-up }\end{array}$ & NA & $\begin{array}{l}\text { Results to meet this objective are discussed in report WTP-RPT-188 and } \\
\text { WTP-RPT-197. }\end{array}$ \\
\hline $\begin{array}{l}\text { Cross-flow Ultrafiltration: Monitor } \\
\text { cross-flow filter performance at } \\
\text { engineering- and laboratory-scale } \\
\text { to determine scale-up }\end{array}$ & Y & $\begin{array}{l}\text { Tests were conducted at the laboratory- and PEP scale with a Hanford tank } \\
\text { waste simulant at low and high solids concentrations. The test conditions, } \\
\text { results, and scale-up factor analysis are reported in Section } 5 \text { of this report. } \\
\text { For the low-solids concentrations, the current scaling tests indicate that a } \\
\text { scaling factor of } 1.0 \text { provides a conservative estimate of scaled filter flux } \\
\text { (where the scaling factor was defined as the ratio of engineering-scale } \\
\text { filter flux to bench-scale filter flux). For the high-solids concentrations, } \\
\text { the scaling factor for dewatering operations is statistically similar (1.0). } \\
\text { Here, scaling factors are based on ratios of parameters that characterize the } \\
\text { dewatering behavior of the simulant slurry. }\end{array}$ \\
\hline $\begin{array}{l}\text { Slurry wash process: Determine the } \\
\text { post caustic and oxidative leaching } \\
\text { slurry wash efficiencies }\end{array}$ & NA & $\begin{array}{l}\text { Results to meet this objective are discussed in report WTP-RPT-187 and } \\
\text { WTP-RPT-197. }\end{array}$ \\
\hline $\begin{array}{l}\text { Process Integration: Evaluate the } \\
\text { chemical addition, filter operation } \\
\text { cycle performance, and pressure } \\
\text { pot operations. Also perform mass } \\
\text { balances for aluminum, chromium, } \\
\text { manganese, sodium, hydroxide, } \\
\text { oxalate, phosphate, sulfate, and } \\
\text { water and monitor permeates for } \\
\text { post filtration precipitation. }\end{array}$ & NA & Results to meet this objective are discussed in WTP-RPT-197 \\
\hline $\begin{array}{l}\text { Monitor the performance of the } \\
\text { recirculation system pumps, filters, } \\
\text { and heat exchanger to support } \\
\text { Engineering fabrication decisions } \\
\text { for these components. }\end{array}$ & NA & $\begin{array}{l}\text { The data required to meet this objective were provided on compact discs } \\
\text { transmitted in the following reference: Letter from GH Beeman to H } \\
\text { Hazen, "Subcontract No. 24590-QL-HC9-WA49-00001, Project No. } \\
53569 \text { (WA-024) Engineering Ties Data Transmittal: The electronic file } \\
\text { enclosed with this letter has been reviewed for technical accuracy per the } \\
\text { QA program, WTP/RPP-MOA-PNNL-00392, dated 4/10/09. }\end{array}$ \\
\hline
\end{tabular}




\section{Test Exceptions}

The test exceptions are provided in Table S.5.

Table S.5. Test Exceptions

\begin{tabular}{|c|c|}
\hline Test Exceptions & Description of Test Exceptions \\
\hline $\begin{array}{l}\text { 1) } 24590 \text {-PTF-TEF-RT-08- } \\
\text { 00002 incorporated into ICN-1 } \\
\text { to Test Plan TP-RPP-WTP-506. }\end{array}$ & $\begin{array}{l}\text { This test exception: } \\
\text { 1. Added a stage during the filter conditioning section of the functional test } \\
\text { where the simulant slurry is concentrated from approximately } 5 \mathrm{wt} \% \text { solids to } \\
20 \mathrm{wt} \% \text { solids in one operation. This is in addition to the previously specified } \\
\text { low solids filter and high solids filter testing. } \\
\text { 2. Documented the Joint Test Group (JTG) decision regarding the number of } \\
\text { replicate samples to be collected at various processing times } \\
\text { 3. Revised the terminology specifying the Coriolis densitometer (CD) sample } \\
\text { locations changed to be consistent with PEP operating procedures. Renamed } \\
\text { the "center" array to "inner." } \\
\text { 4. The sampling specified in the low solids filtration test over specifies the } \\
\text { sample collection timing required. The technical requirement is to get } 30 \\
\text { unique samples. The sampling schedule specified is not required to achieve } \\
\text { this test objective. }\end{array}$ \\
\hline $\begin{array}{l}\text { 2) } 24590 \text {-PTF-TEF-RT-09- } \\
00001 \text { incorporated into ICN-2 } \\
\text { and ICN-3 to Test Plan TP-RPP- } \\
\text { WTP-506. }\end{array}$ & $\begin{array}{l}\text { 1. In several steps, the sampling location was changed from the filer loop in-line } \\
\text { location to a middle-low CD sample loop location in the UFP-T02A vessel. } \\
\text { This change impacted sampling in the functional and all integrated tests (ref } \\
\text { CCN 187749). } \\
\text { 2. Added a step to the shakedown/functional test (step A.1.31) to add sodium } \\
\text { permanganate to UFP-VSL-T02A to assess possible foaming issue (ref CCN } \\
\text { 187749). } \\
\text { 3. Changed location of second sample for parallel CUF testing from the in-line } \\
\text { filter loop to the middle-low CD port in the UFP-VSL-T02A (step A.1.10; } \\
\text { Functional Test) (ref CCN 187749). } \\
\text { 4. Collected samples for parallel laboratory leaching test before and after caustic } \\
\text { addition in UFP-VSL-T01A (A.1.20; Functional test) and UFP-VSL-T02A } \\
\text { (step A.1.15; Functional test), and in the integrated test steps (B.1.2; } \\
\text { Integrated Test A, B.2.6; Integrated Tests B/D) (ref CCN: 192734). } \\
\text { 5. Deleted reconfiguration of the filter loop to bypass UFP-VSL-T02A and } \\
\text { circulate flush water with UFP-PMP-T02A and/or UFP-PMP-43A to allow } \\
\text { collection of a representative in-line sample. This step (step A.1.17; } \\
\text { Functional test) could not be done under the operating restrictions in place on } \\
\text { the operation of the filter loop. (CCN: 192734) } \\
\text { 6. Eliminated step A.1.25 (filter-loop by-pass test with tracer) from the } \\
\text { functional test. This test was conducted after the completion of Integrated } \\
\text { Test B (ref CCN 187753). } \\
\text { 7. Modified step A.1.29 (Functional test) to eliminate the removal of solids } \\
\text { from UFP-VSL-T02A before the high solids filter test. This step was not } \\
\text { needed as the amount of solids is less than anticipated (ref CCN 187752). } \\
\text { 8. Modified step A.1.30 (Functional test) to include five filter backpulses before } \\
\text { starting the high-solids filter test (ref CCN 187752) }\end{array}$ \\
\hline
\end{tabular}


Table S.5. Test Exceptions

\begin{tabular}{|c|c|}
\hline Test Exceptions & Description of Test Exceptions \\
\hline & 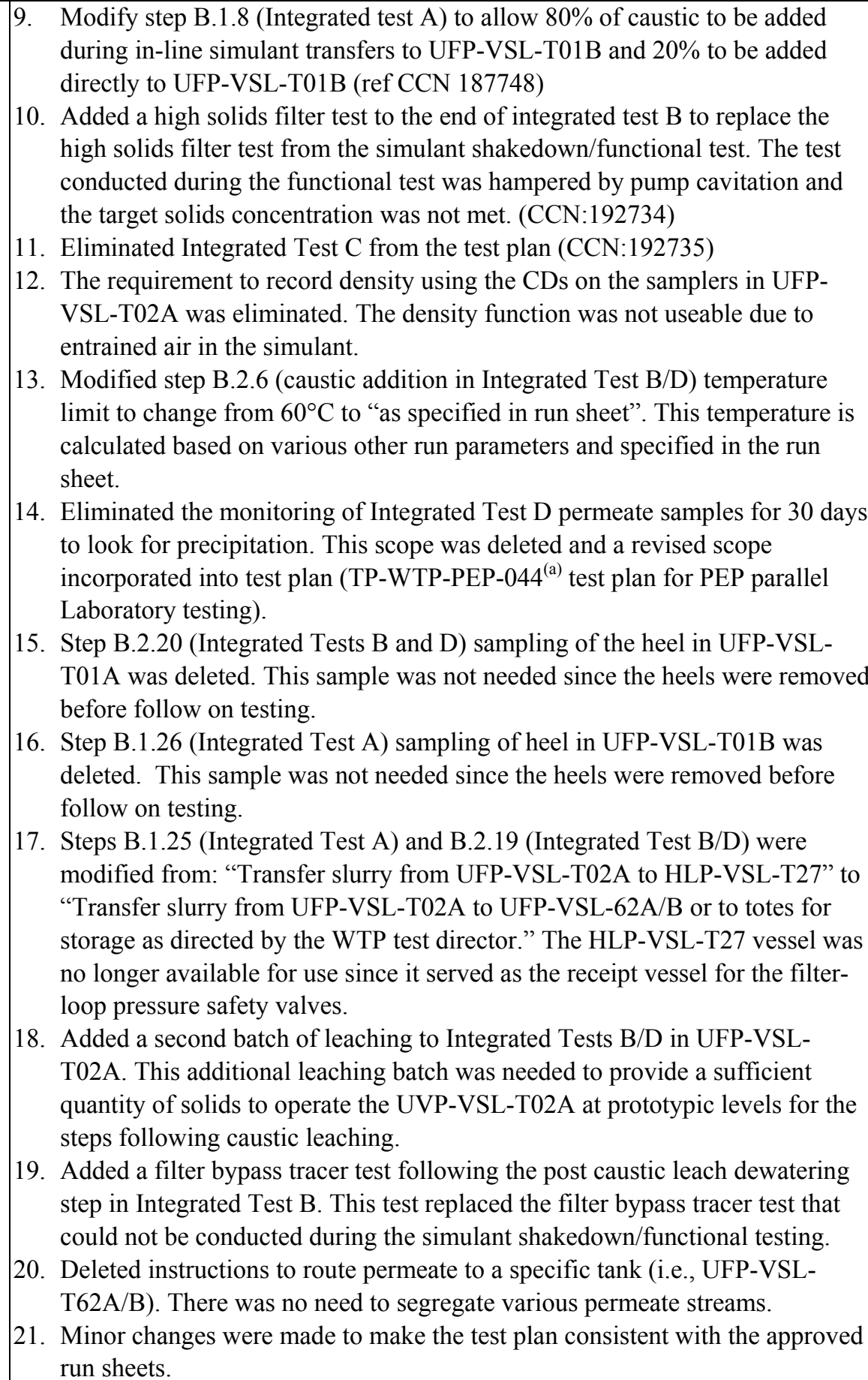 \\
\hline $\begin{array}{l}\text { 3) } 24590 \text {-WTP-TEF-RT-09- } \\
00003 \text { incorporated into ICN-1 }\end{array}$ & $\begin{array}{l}\text { This test exception specified activities to be performed with permeate samples } \\
\text { obtained from Integrated Test D. The Integrated Test D permeate samples were }\end{array}$ \\
\hline
\end{tabular}

(a) RL Russell. 2008. "Test Plan for the PEP Parallel Laboratory Testing." TP-WTP-PEP-044, Rev. 0.2, Pacific Northwest National Laboratory, Richland, Washington. 
Table S.5. Test Exceptions

\begin{tabular}{|c|c|}
\hline Test Exceptions & \begin{tabular}{|c|} 
Description of Test Exceptions \\
\end{tabular} \\
\hline to test plan TP-WTP-PEP-044. & $\begin{array}{l}\text { originally stored in a temperature controlled environment and then moved to a } \\
\text { location with a reduced temperature where precipitation was likely to occur. The test } \\
\text { exception requested that the approximate size distribution of the solids be measured in } \\
\text { several ( } 3 \text { or 4) selected PEP samples from Integrated Test D using polarized light } \\
\text { microscopy (PLM). Size-calibrated photographs should be provided along with the } \\
\text { analysis. If possible, record the mineral identification of the solids phase(s) along with } \\
\text { the particle-size distribution. Selection of the samples will be made by WTP personnel } \\
\text { in consultation with the subcontractor, and will be based in part on observation of } \\
\text { which samples contain the most solids or appear to contain different types of solids. } \\
\text { Repeat the size distribution analysis approximately one week after the initial } \\
\text { measurements to determine whether there was a significant change in crystal size, } \\
\text { habit, or composition. } \\
\text { Perform each size distribution analysis by measuring the diameter (or length and } \\
\text { width for elongated crystals) of approximately } 100 \text { individual particles in each sample. } \\
\text { The size may be measured either on the microscope slide, using a calibrated ocular } \\
\text { scale, or on the size-calibrated photographs. The program recognizes the limitations of } \\
\text { the statistical significance of a size distribution measurement based on such a small } \\
\text { population. This test exception did not affect any of the existing test plan objectives. }\end{array}$ \\
\hline $\begin{array}{l}\text { 4) 24590-PTF-TEF-RT-09- } \\
\text { 00002 Rev 0, incorporated into } \\
\text { ICN-4 to Test Plan TP-RPP- } \\
\text { WTP-506 }\end{array}$ & 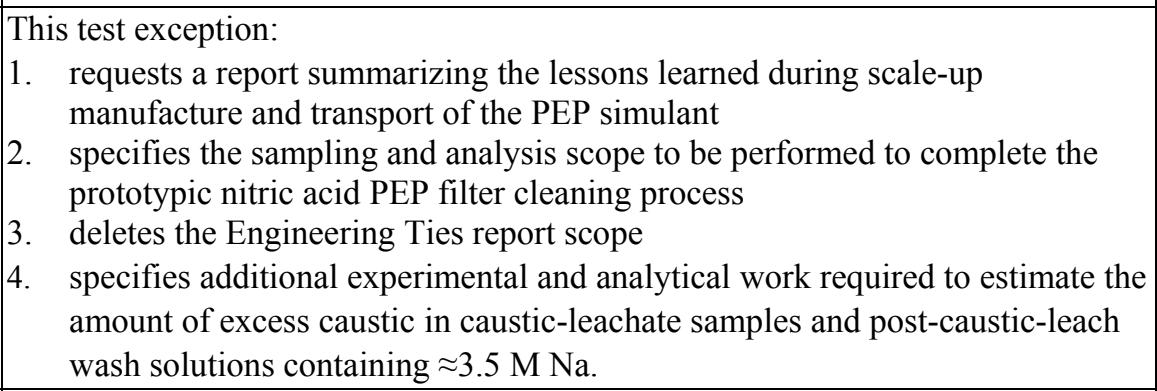 \\
\hline $\begin{array}{l}\text { 5) } 24590 \text {-WTP-TEF-RT-09- } \\
\text { 00001 Rev } 1 \text { incorporated into } \\
\text { ICN-2 to test plan TP-WTP- } \\
\text { PEP-044 }\end{array}$ & $\begin{array}{l}\text { This test exception specifies additional work to be conducted with caustic leach } \\
\text { solutions and post-caustic-leach washing permeate samples obtained from PEP } \\
\text { Integrated Tests A, B, and D. It contains the following tasks: } \\
\text { 1) Determination of precipitate mineralogy, precipitate phase compositions, and } \\
\text { solution saturation composition. } \\
\text { 2) Determination of rate of approach to saturation concentrations. } \\
\text { 3) Identification and characterization of precipitates formed in post-caustic leach } \\
\text { filtrate. } \\
\text { 4) Determination of the dilution required to re-dissolve the precipitate. } \\
\text { 5) Determination of super-saturation in post-caustic leach filtrates from Test B } \\
\text { in the PEP. } \\
\text { 6) Determine the effects of blending during the post-caustic-leach dewatering } \\
\text { and wash cycle. }\end{array}$ \\
\hline
\end{tabular}


As documented in the PEP test plan, the deviations from the test specification are provided in Table S.6.

Table S.6. Deviations from Test Specification

\begin{tabular}{|c|c|}
\hline Test Specification Reference & Exception Taken \\
\hline $\begin{array}{l}\text { Section 6.4.4 "Analytical measurements will be } \\
\text { made in conformance to the Guidelines for } \\
\text { Performing Chemical Physical, and Rheological } \\
\text { Properties Measurements }{ }^{\text {(a) }} \text { as applicable." }\end{array}$ & $\begin{array}{l}\text { Three method exceptions are required under this test plan: } \\
\text { 1. Caustic leach and oxidative leach samples taken during this } \\
\text { testing must be separated more quickly than the standard } \\
\text { method using syringes. This testing will use a modified } \\
\text { method using a shorter centrifuge time and applying higher } \\
g \text { forces (e.g. } 4000 \mathrm{~g} \text { vs. } 1000 \mathrm{~g} \text { ). } \\
\text { Impact on results: If the standard method were used, the } \\
\text { longer time could very well lead to greater precipitation } \\
\text { and inaccurate results. Laboratory testing will be } \\
\text { conducted with simulants to confirm that this method of } \\
\text { sample handling is adequate. } \\
\text { 2. Densities of samples smaller than } 10 \mathrm{~mL} \text { can only be } \\
\text { established within two significant figures of accuracy. } \\
\text { Density measurements for this test plan require greater } \\
\text { accuracy. Therefore, a more accurate method employing a } \\
\text { pycnometer will be utilized. } \\
\text { Impact on results: The change to a pycnometer will } \\
\text { generate more precise results than the standard method. } \\
\text { The main impact is expected to be on analysis time. The } \\
\text { pycnometer method will be slower. } \\
\text { 3. The process for determining the wt } \% \text { UDS content of the } \\
\text { slurries will in some cases be determined with the use of a } \\
\text { moisture analyzer. In addition, the method of drying } \\
\text { samples will be modified to allow the use of glass fiber } \\
\text { filters to aid in drying the samples. } \\
\text { Impact on results: Both modifications are intended to } \\
\text { decrease the time required to obtain results. }\end{array}$ \\
\hline
\end{tabular}

\section{Results and Performance Against Success Criteria}

The PEP system tests were designed to generate the data necessary to:

- Provide engineering-scale system performance data. This information is used to support the WTP computer process models projections of the waste processing campaign

- Confirm the operability and functionality of UFP system components.

The R\&T success criteria for achieving these objectives are discussed in Table S.7. The success criteria for the entire PEP testing program are provided with discussion limited to the success criteria covered by the scope of this report. The success criteria not addressed in this report are shaded in gray.

(a) GL Smith and K Prindiville. May 20, 2002. Guidelines for Performing Chemical, Physical, and Rheological Properties Measurements. 24590-WTP-GPG-RTD-001, Rev 0, Bechtel National, Inc., Richland, Washington. 
Table S.7. Success Criteria

\begin{tabular}{|c|c|}
\hline Success Criteria & How Testing Did or Did Not Meet Success Criteria \\
\hline \multicolumn{2}{|l|}{ UFP System Process Performance } \\
\hline $\begin{array}{l}\text { Measure the aluminum leaching } \\
\text { performance of the PEP and laboratory } \\
\text { systems as a function of time under WTP } \\
\text { UFP- } 1 \text { and UFP- } 2 \text { projected leaching } \\
\text { conditions at bounding high and low } \\
\text { process temperatures (nominally } 100^{\circ} \mathrm{C} \\
\text { and } 80^{\circ} \mathrm{C} \text { ). }\end{array}$ & $\begin{array}{l}\text { Results to meet this success criterion are discussed in report WTP- } \\
\text { RPT-186 and WTP-RPT-197. }\end{array}$ \\
\hline $\begin{array}{l}\text { Compare aluminum leach performance in } \\
\text { UFP- } 1 \text { where all of the } \mathrm{NaOH} \text { is added in- } \\
\text { line to the case where a fraction of the total } \\
\mathrm{NaOH} \text { is added directly to the tank. }\end{array}$ & $\begin{array}{l}\text { Results to meet this success criterion are discussed in report WTP- } \\
\text { RPT-197. }\end{array}$ \\
\hline $\begin{array}{l}\text { Measure chromium leaching performance } \\
\text { in the PEP and laboratory systems as a } \\
\text { function of time at the WTP projected } \\
\text { conditions in UFP-2 for both the UFP-1 } \\
\text { and UFP-2 aluminum leaching flowsheets. }\end{array}$ & $\begin{array}{l}\text { Results to meet this success criterion are discussed in report WTP- } \\
\text { RPT-188 and WTP-RPT-197. }\end{array}$ \\
\hline $\begin{array}{l}\text { Evaluate the process control strategy for } \\
\text { specification of required reagent additions } \\
\text { including } \mathrm{NaOH}, \mathrm{NaMnO}_{4} \text {, and wash } \\
\text { solutions provided in the Pretreatment } \\
\text { Engineering Platform (PEP) Phase } 1 \\
\text { Testing Process Description. }\end{array}$ & $\begin{array}{l}\text { Results to meet this success criterion are discussed in report WTP- } \\
\text { RPT-188 for Integrated Tests A and B. Additional discussion and } \\
\text { results for Integrated Test D are discussed in WTP-RPT-197. }\end{array}$ \\
\hline $\begin{array}{l}\text { Measure the filter system performance at } \\
\text { the nominal flow velocity and TMPs for } \\
\text { the solids concentration and washing } \\
\text { stages for the UFP-1 and UFP-2 aluminum } \\
\text { leaching flowsheets. }\end{array}$ & $\begin{array}{l}\text { Results to meet this success criterion are discussed in report WTP- } \\
\text { RPT-197. }\end{array}$ \\
\hline $\begin{array}{l}\text { Evaluate the control strategy for make-up } \\
\text { additions from UFP-VSL-00001A/B to } \\
\text { UFP-VSL-00002A/B during initial } \\
\text { dewatering process. }\end{array}$ & $\begin{array}{l}\text { Results to meet this success criterion are discussed in report WTP- } \\
\text { RPT-197. }\end{array}$ \\
\hline $\begin{array}{l}\text { Measure the wash water volumes required } \\
\text { to remove or reduce the free hydroxide } \\
\text { following the aluminum leaching stage and } \\
\text { dissolved chromium after the oxidative } \\
\text { leaching process to the specified } \\
\text { concentrations. }\end{array}$ & $\begin{array}{l}\text { Results to meet this success criterion are discussed in report WTP- } \\
\text { RPT-187 and WTP-RPT-197. }\end{array}$ \\
\hline $\begin{array}{l}\text { Perform mass balances for selected } \\
\text { constituents, including aluminum, } \\
\text { chromium, manganese, sodium, hydroxide, } \\
\text { oxalate, phosphate, sulfate, and water to } \\
\text { evaluate leaching and washing process } \\
\text { performance. }\end{array}$ & $\begin{array}{l}\text { Results to meet this success criterion are discussed for Cr in the } \\
\text { oxidative leaching process for Integrated Tests A and B in report } \\
\text { WTP-RPT-188 and are fully discussed for all constituents in report } \\
\text { WTP-RPT-197. }\end{array}$ \\
\hline $\begin{array}{l}\text { Measure solids distribution under scaled } \\
\text { mixing conditions before and after caustic } \\
\text { leaching evolutions. }\end{array}$ & $\begin{array}{l}\text { Results to meet this success criterion are discussed in report WTP- } \\
\text { RPT-197. }\end{array}$ \\
\hline
\end{tabular}


Table S.7. Success Criteria

\begin{tabular}{|c|c|}
\hline Success Criteria & How Testing Did or Did Not Meet Success Criteria \\
\hline $\begin{array}{l}\text { Measure the rheology of the slurry } \\
\text { simulant and shear strength of the settled } \\
\text { solids before and after each leaching and } \\
\text { washing unit operation and following final } \\
\text { concentration. }\end{array}$ & $\begin{array}{l}\text { Results to meet this success criterion are discussed in report WTP- } \\
\text { RPT-197. }\end{array}$ \\
\hline $\begin{array}{l}\text { Estimate the quantity of excess hydroxide } \\
\text { added in the process that may not be } \\
\text { needed to keep aluminate in solution } \\
\text { following filtration. }\end{array}$ & $\begin{array}{l}\text { Results to meet this success criterion are discussed in report WTP- } \\
\text { RPT-197. }\end{array}$ \\
\hline $\begin{array}{l}\text { Collect and retain permeate samples for } \\
\text { extended precipitation studies (including } \\
\text { permeate/simulated supernatant blended } \\
\text { cases) from each concentration cycle. }\end{array}$ & $\begin{array}{l}\text { Samples were collected and retained for extended precipitation } \\
\text { studies. The results of the precipitation studies are discussed in WTP- } \\
\text { RPT-197, WTP-RPT-200 and WTP-RPT-205. }\end{array}$ \\
\hline \multicolumn{2}{|l|}{ UFP System operability and functionality } \\
\hline $\begin{array}{l}\text { Verify that the dual, in series pump } \\
\text { configuration is controllable and maintains } \\
\text { the required slurry velocity and pressures } \\
\text { for ultrafilter operation. }\end{array}$ & $\begin{array}{l}\text { The data required to meet this success criterion were provided on } \\
\text { Compact Disks transmitted in the following reference: Letter from } \\
\text { GH Beeman to H Hazen, "Subcontract No. 24590-QL-HC9-WA49- } \\
\text { 00001, Project No. 53569 (WA-024) Engineering Ties Data } \\
\text { Transmittal: The Electronic File Enclosed With This letter Has Been } \\
\text { Reviewed For Technical Accuracy Per the QA Program", WTP/RPP- } \\
\text { MOA-PNNL-00392, dated 4/10/09. }\end{array}$ \\
\hline $\begin{array}{l}\text { Measure the operating characteristics for } \\
\text { the cooling heat exchanger for the UFP- } \\
\text { VSL-00002 filter recirculation loop } \\
\text { (temperature changes as a function of flow } \\
\text { to determine how to achieve the desired } \\
\text { performance in the PTF analog). }\end{array}$ & $\begin{array}{l}\text { The data required to meet this success criterion were provided on } \\
\text { Compact Disks transmitted in the following reference: Letter from } \\
\text { GH Beeman to H Hazen, "Subcontract No. 24590-QL-HC9-WA49- } \\
\text { 00001, Project No. } 53569 \text { (WA-024) Engineering Ties Data } \\
\text { Transmittal: The Electronic File Enclosed With This letter Has Been } \\
\text { Reviewed For Technical Accuracy Per the QA Program", WTP/RPP- } \\
\text { MOA-PNNL-00392, dated 4/10/09. }\end{array}$ \\
\hline $\begin{array}{l}\text { Confirm whether the WTP process control } \\
\text { strategies for ultrafilter system filling, } \\
\text { operating, backpulsing, draining, flushing, } \\
\text { and cleaning are adequate for } \\
\text { stable operation. Provide to WTP data to } \\
\text { determine whether backpulsing is a } \\
\text { required and effective means of restoring } \\
\text { the filter permeate rates to ensure } \\
\text { production throughput is maintained and } \\
\text { whether operation of the backpulse system } \\
\text { induces any process or equipment } \\
\text { operations issues. }\end{array}$ & $\begin{array}{l}\text { Results to meet this success criterion are discussed in report WTP- } \\
\text { RPT-197. }\end{array}$ \\
\hline $\begin{array}{l}\text { Use only the process information and data } \\
\text { available to the WTP PTF operating staff } \\
\text { during WTP operations (e.g., caustic and } \\
\text { permanganate addition volumes, permeate } \\
\text { mass balances for solids concentration, } \\
\text { etc.) to operate the PEP. }\end{array}$ & $\begin{array}{l}\text { Results to meet this success criterion are discussed in report WTP- } \\
\text { RPT-197. }\end{array}$ \\
\hline Confirm whether the elevated temperature & Results to meet this success criterion are discussed in report WTP- \\
\hline
\end{tabular}


Table S.7. Success Criteria

\begin{tabular}{|c|c|}
\hline Success Criteria & How Testing Did or Did Not Meet Success Criteria \\
\hline $\begin{array}{l}\text { PJM operating strategy is adequate for } \\
\text { stable PEP and WTP operation. }\end{array}$ & RPT-197. \\
\hline $\begin{array}{l}\text { Measure the heat-up rate and } \\
\text { controllability of the PEP UFP-VSL- } 00001 \\
\text { and UFP-VSL-00002 vessels and the } \\
\text { cooling performance for UFP vessels. }\end{array}$ & $\begin{array}{l}\text { Results to meet this success criterion are discussed in report WTP- } \\
\text { RPT-197. }\end{array}$ \\
\hline $\begin{array}{l}\text { Measure the performance of the in-line } \\
\text { addition of process chemicals into the } \\
\text { simulated wastes and determine the extent } \\
\text { of blending in the process vessels. }\end{array}$ & $\begin{array}{l}\text { Results to meet this success criterion are discussed in report WTP- } \\
\text { RPT-197. }\end{array}$ \\
\hline $\begin{array}{l}\text { Monitor ultrafilter performance (to include } \\
\text { visual inspection of the filter tubes, tube } \\
\text { sheets, and heads from an ultrafilter for } \\
\text { any evidence of flow mal-distribution } \\
\text { and/or solids buildup at least once during } \\
\text { Phase 1). }\end{array}$ & $\begin{array}{l}\text { Results to meet this success criterion are discussed in report WTP- } \\
\text { RPT-197. }\end{array}$ \\
\hline $\begin{array}{l}\text { Measure, record, and control ultrafiltration } \\
\text { temperature, TMP, and slurry flow during } \\
\text { filter-loop operations. }\end{array}$ & $\begin{array}{l}\text { Results to meet this success criterion are provided in Section } 5 \text { for the } \\
\text { low and high solids filter tests. Results to meet this success criterion } \\
\text { for other process steps are discussed in the run reports for each of the } \\
\text { integrated tests. }\end{array}$ \\
\hline $\begin{array}{l}\text { Record any solids accumulations observed } \\
\text { during any operating stage or maintenance } \\
\text { evolution. }\end{array}$ & $\begin{array}{l}\text { Results to meet this success criterion are discussed in report WTP- } \\
\text { RPT-197. }\end{array}$ \\
\hline $\begin{array}{l}\text { Monitor the permeate production rate of } \\
\text { each ultrafilter assembly in operation. }\end{array}$ & $\begin{array}{l}\text { The data to meet this success criterion for the low and high solids } \\
\text { filter tests are reported in Section } 5 \text {. The remaining results are } \\
\text { reported in the run reports for each integrated test and report WTP- } \\
\text { RPT-197. }\end{array}$ \\
\hline $\begin{array}{l}\text { Record operating time of each ultrafilter } \\
\text { assembly. }\end{array}$ & $\begin{array}{l}\text { Results to meet this success criterion are discussed in report WTP- } \\
\text { RPT-197. }\end{array}$ \\
\hline $\begin{array}{l}\text { Record each ultrafilter assembly cleaning } \\
\text { event (backpulse, flush, chemical cleaning, } \\
\text { etc.). }\end{array}$ & $\begin{array}{l}\text { Results to meet this success criterion are discussed in report WTP- } \\
\text { RPT-197. }\end{array}$ \\
\hline $\begin{array}{l}\text { Evaluation of the pulse pot operation and } \\
\text { backpulse operation strategies contained in } \\
\text { Pretreatment Engineering Platform (PEP) } \\
\text { Phase } 1 \text { Testing Process Description. }\end{array}$ & $\begin{array}{l}\text { Results to meet this success criterion are discussed in report WTP- } \\
\text { RPT-197. }\end{array}$ \\
\hline $\begin{array}{l}\text { Evaluate permeate and permeate blends for } \\
\text { precipitation of solids, particularly } \\
\text { aluminum and oxalate solids. }\end{array}$ & $\begin{array}{l}\text { Results to meet this success criterion are discussed in reports WTP- } \\
\text { RPT-197, WTP-RPT-200 and WTP-RPT-205. }\end{array}$ \\
\hline
\end{tabular}

\section{Quality Requirements}

The PNNL Quality Assurance Program is based upon the requirements as defined in the U.S. Department of Energy (DOE) Order 414.1C, Quality Assurance and 10 CFR 830, Energy/Nuclear Safety Management, Subpart A -- Quality Assurance Requirements (a.k.a. the Quality Rule). PNNL has chosen to implement the following consensus standards in a graded approach: 
- ASME NQA-1-2000, Quality Assurance Requirements for Nuclear Facility Applications, Part 1, Requirements for Quality Assurance Programs for Nuclear Facilities

- ASME NQA-1-2000, Part II, Subpart 2.7, Quality Assurance Requirements for Computer Software for Nuclear Facility Applications

- ASME NQA-1-2000, Part IV, Subpart 4.2, Graded Approach Application of Quality Assurance Requirements for Research and Development.

The procedures necessary to implement the requirements are documented in PNNL's Standards-Based Management System (SBMS).

The RPP-WTP quality requirements are implemented by performing work in accordance with the River Protection Project - Waste Treatment Plant Support Program (RPP-WTP) Quality Assurance Plan (RPP-WTP-QA-001, QAP). Work was performed to the quality requirements of NQA-1-1989 Part I, Basic and Supplementary Requirements, NQA-2a-1990, Part 2.7, and DOE/RW-0333P, Rev 13, Quality Assurance Requirements and Descriptions (QARD), as applicable. These quality requirements are implemented through the River Protection Project - Waste Treatment Plant Support Program (RPPWTP) Quality Assurance Manual (RPP-WTP-QA-003, QAM). The requirements of DOE/RW-0333P Rev 13, Quality Assurance Requirements and Descriptions (QARD), and 10 CFR 830 Subpart A were not required for this work.

The RPP-WTP addresses internal verification and validation activities by conducting an independent technical review of the final data report in accordance with RPP-WTP's procedure QA-RPP-WTP-604. This review procedure is part of PNNL's RPP-WTP Quality Assurance Manual (RPP-WTP-QA-003). Following this procedure, a technical review would verify that the reported results are traceable, that inferences and conclusions are soundly based, and the reported work satisfies the objectives.

\section{R\&T Test Conditions}

The research and technology (R\&T) test conditions, as defined in the Test Specification, are summarized in Table S.8. The R\&T test conditions for the entire PEP testing program are provided with discussion limited to the R\&T test conditions covered by the scope of this report. The R\&T test conditions not addressed in this report are shaded in gray. 
Table S.8. R\&T Test Conditions

\begin{tabular}{|c|c|}
\hline List R\&T Test Conditions & Were Test Conditions Followed? \\
\hline \multicolumn{2}{|l|}{ General Requirements } \\
\hline $\begin{array}{l}\text { Perform mass balances for selected constituents; } \\
\text { including aluminum, chromium, manganese, sodium, } \\
\text { hydroxide, oxalate, phosphate, sulfate, and water to } \\
\text { evaluate leaching and washing process performance. }\end{array}$ & $\begin{array}{l}\text { This R\&T test condition is discussed for } \mathrm{Cr} \text { in the } \\
\text { oxidative leach process in Integrated Tests A and B in } \\
\text { WTP-RPT-188 and are fully discussed in report WTP- } \\
\text { RPT-197. }\end{array}$ \\
\hline $\begin{array}{l}\text { Evaluate ultrafilter performance (to include visual } \\
\text { inspection of the filter tubes, tube sheets, and heads } \\
\text { from an ultrafilter for any evidence of flow mal- } \\
\text { distribution and/or solids buildup or evidence of } \\
\text { potential failure). }\end{array}$ & $\begin{array}{l}\text { This R\&T test condition is discussed in report WTP- } \\
\text { RPT-197. }\end{array}$ \\
\hline $\begin{array}{l}\text { Assess the blending achieved during in-line additions of } \\
\text { leaching and washing solutions. }\end{array}$ & $\begin{array}{l}\text { In-line addition of wash water during Integrated Tests } \\
\text { A and B are assessed in report WTP-RPT-187 and are } \\
\text { fully discussed in report WTP-RPT-197. }\end{array}$ \\
\hline $\begin{array}{l}\text { Record any solids accumulations observed during any } \\
\text { operating stage or maintenance evolution (e.g., } \\
\text { photography, particle-size distribution). }\end{array}$ & $\begin{array}{l}\text { This R\&T test condition is discussed in report WTP- } \\
\text { RPT-197. }\end{array}$ \\
\hline \multicolumn{2}{|l|}{ Leaching Operations } \\
\hline $\begin{array}{l}\text { Maintain caustic leaching temperature at the required } \\
\text { setpoint and record steam usage to remain in the } \\
\text { temperature range }\end{array}$ & $\begin{array}{l}\text { This R\&T test condition is discussed in report WTP- } \\
\text { RPT-186 and WTP-RPT- } 197 .\end{array}$ \\
\hline $\begin{array}{l}\text { Maintain oxidative leaching temperature at the required } \\
\text { setpoint. }\end{array}$ & $\begin{array}{l}\text { This R\&T test condition is discussed in report WTP- } \\
\text { RPT-188 and WTP-RPT- } 197 .\end{array}$ \\
\hline $\begin{array}{l}\text { Obtain periodic samples during the leaching operations } \\
\text { to monitor the amount of aluminum or chromium that } \\
\text { has dissolved and concentrations of the reactants and } \\
\text { products in the liquid fraction in the vessel. }\end{array}$ & $\begin{array}{l}\text { This R\&T test condition is discussed in reports WTP- } \\
\text { RPT-186, WTP-RPT-188, and WTP-RPT-197. }\end{array}$ \\
\hline $\begin{array}{l}\text { Provide data to demonstrate the WTP process control } \\
\text { strategy for the caustic and permanganate addition. }\end{array}$ & $\begin{array}{l}\text { This R\&T test condition is discussed in report WTP- } \\
\text { RPT- } 197 .\end{array}$ \\
\hline $\begin{array}{l}\text { Measure the rheology of the slurry simulant and shear } \\
\text { strength of the settled solids before and following each } \\
\text { leaching unit operation. }\end{array}$ & $\begin{array}{l}\text { This R\&T test condition is discussed in report WTP- } \\
\text { RPT-197. }\end{array}$ \\
\hline \multicolumn{2}{|l|}{ Concentration Operations } \\
\hline $\begin{array}{l}\text { Monitor the permeate production rate of each ultrafilter } \\
\text { assembly in operation. }\end{array}$ & $\begin{array}{l}\text { Yes. Permeate production rates were monitored for the } \\
\text { low and high solids filter tests and are presented in } \\
\text { Section 5. Permeate rates for the other process steps } \\
\text { are presented in the individual run reports and WTP- } \\
\text { RPT-197. }\end{array}$ \\
\hline Record operating time of each ultrafilter assembly. & $\begin{array}{l}\text { This R\&T test condition is discussed in report WTP- } \\
\text { RPT-197. }\end{array}$ \\
\hline $\begin{array}{l}\text { Record each ultrafilter assembly 'cleaning' event } \\
\text { (backpulse, flush, chemical cleaning, etc.). }\end{array}$ & $\begin{array}{l}\text { This R\&T test condition is discussed in report WTP- } \\
\text { RPT- } 197 .\end{array}$ \\
\hline $\begin{array}{l}\text { Confirm pulse pot operation and backpulse operation } \\
\text { strategies. }\end{array}$ & $\begin{array}{l}\text { This R\&T test condition is discussed in report WTP- } \\
\text { RPT- } 197 .\end{array}$ \\
\hline
\end{tabular}


Table S.8. R\&T Test Conditions

\begin{tabular}{|c|c|}
\hline List R\&T Test Conditions & Were Test Conditions Followed? \\
\hline $\begin{array}{l}\text { Control ultrafiltration temperature, TMP, and slurry } \\
\text { flow as specified in test specific run sheets. }\end{array}$ & $\begin{array}{l}\text { Yes. The R\&T test conditions for the low solids filter } \\
\text { test were met. In the PEP high-solids test, the AV of } \\
\text { the slurry in the filter tubes ranged from about } 13.7 \text { to } \\
17 \mathrm{ft} / \mathrm{sec} \text {, which exceeded the specified range of } 15.0 \\
\pm 1.4 \mathrm{ft} / \mathrm{sec} \text { (see Section 5). This was caused by } \\
\text { difficulties controlling the pumps due to changes in } \\
\text { the slurry rheology. Near the end of the PEP high } \\
\text { solids filter test, the temperature exceeded the } \\
\text { specified range of } 25 \pm 2^{\circ} \mathrm{C} \text { due to erroneous } \\
\text { temperature readings from the resistance temperature } \\
\text { detector (RTD) controlling the cooling heat } \\
\text { exchanger. This was caused by stagnation of the thick } \\
\text { slurry in the thermowells, which prevented the RTDs } \\
\text { from measuring the process stream temperature. The } \\
\text { impact of these deviations is to increase the } \\
\text { uncertainty of the high-solids scaling factor. } \\
\text { Discussion of the R\&T test conditions for other } \\
\text { filtration steps are discussed in the run reports for the } \\
\text { individual tests and report WTP-RPT- } 197 \text {. }\end{array}$ \\
\hline $\begin{array}{l}\text { Collect and retain permeate samples for extended } \\
\text { precipitation studies (including permeate/simulated } \\
\text { supernatant blended cases) from each concentration } \\
\text { cycle. }\end{array}$ & $\begin{array}{l}\text { Samples were collected and retained for extended } \\
\text { precipitation studies. The results of the precipitation } \\
\text { studies are discussed in WTP-RPT-197, WTP-RPT- } \\
200 \text { and WTP-RPT-205. }\end{array}$ \\
\hline $\begin{array}{l}\text { Demonstrate WTP ultrafiltration system control scheme } \\
\text { in normal operating modes (e.g., fill and startup, } \\
\text { operation, backpulsing, flush and drain, cleaning and } \\
\text { return to service). }\end{array}$ & $\begin{array}{l}\text { This R\&T test condition is discussed in report WTP- } \\
\text { RPT-197. }\end{array}$ \\
\hline \multicolumn{2}{|l|}{ Washing Operations } \\
\hline $\begin{array}{l}\text { Wash slurries using a washing protocol to be specified } \\
\text { in test specific run sheets. }\end{array}$ & $\begin{array}{l}\text { This R\&T test condition is discussed in report WTP- } \\
\text { RPT-187 and WTP-RPT-197. }\end{array}$ \\
\hline $\begin{array}{l}\text { Sample permeate immediately before each wash } \\
\text { solution addition to monitor washing } \\
\text { performance/efficiency. }\end{array}$ & $\begin{array}{l}\text { This R\&T test condition is discussed in report WTP- } \\
\text { RPT-187 and WTP-RPT- } 197 .\end{array}$ \\
\hline Measure rheology of the washed solids. & $\begin{array}{l}\text { This R\&T test condition is discussed in report WTP- } \\
\text { RPT- } 187 \text { and WTP-RPT- } 197 \text {. }\end{array}$ \\
\hline
\end{tabular}

\section{Simulant Use}

PEP process testing was performed with a non-radioactive aqueous slurry of simulant waste chemicals and solids. The simulant composition and make-up recipe were provided by WTP as documented in Simulant Recommendation for Phase 1 Testing in the Pretreatment Engineering Platform (24590-PTF-RT-08-006 Rev. 0). ${ }^{\text {(a) }}$ Aqueous chemical concentrations were within ranges expected for

(a) PS Sundar. 2008. Simulant Recommendation for Phase 1 Testing in the Pretreatment Engineering Platform. 24590-PTF-RT-08-006 Rev. 0, Bechtel National, Inc., Richland, Washington. 
waste feeds to the PTF except for the hydroxide, oxalate, and phosphate anions. The hydroxide concentration was approximately one standard deviation from the average concentration expected in the feeds to the plant. The oxalate and phosphate components were at their respective solubility limits. The solids components and blend were selected to obtain targeted solids mass loss (aluminum and chromium leaching and oxalate washing) and treatment time. The simulant was not selected to represent any particular Hanford tank waste type.

The simulant was blended from the components listed below. The basis for selecting the individual components and comparison to actual waste behavior is provided where applicable in the indicated references

- Boehmite (for Al) (Russell et al. 2009a)

- Gibbsite (for Al) (Russell et al. 2009b)

- Chrome oxy-hydroxide (CrOOH) slurry (Rapko et al. 2007)

- Sodium oxalate

- Filtration simulant (Russell et al. 2009c)

- Supernate

Because the high-temperature caustic leaching process was found to dissolve significant amounts of the $\mathrm{CrOOH}$ solids, a separate chromium solids simulant was prepared and added to the PEP process after post-caustic leach washing (a non-prototypic addition) in Integrated Tests A and B. In test D, the chromium solids component of the simulant was added to the feed to demonstrate the PTF permanganate addition strategy.

Simulant was procured from NOAH Technologies Corporation (San Antonio, TX). Samples of each simulant batch were characterized to ensure chemical and physical properties requirements were met. Batches of the simulant were procured as follows:

- A 15-gallon trial batch of the blended simulant for laboratory testing to demonstrate the efficacy of the simulant fabrication procedure.

- A 250-gallon scale-up batch of the blended simulant to demonstrate scale-up of the simulant fabrication procedure to an intermediate scale.

- Batches 0,1 , and 2, each nominally 3500 gallons of blended simulant for the shakedown/functional tests and integrated tests $\mathrm{A}$ and $\mathrm{B}$. These batches did not contain the $\mathrm{CrOOH}$ component.

- Batch 3, nominally 1200 gal, for integrated test D. This batch contained the $\mathrm{CrOOH}$ solids component.

- The $\mathrm{CrOOH}$ solids slurry for the shakedown/functional test and tests $\mathrm{A}$ and B was obtained in two separate batches containing nominally 18 and $36 \mathrm{~kg}$ of $\mathrm{Cr}$ as $\mathrm{CrOOH}$. 


\section{Discrepancies and Follow-on Tests}

1) Divergence of filter flux between the filter bundles during the backpulse sequence at PEP is a repeatable phenomenon without an established cause. Designing a set of tests to study this phenomenon by itself could provide an answer to the discrepancies in filter flux.

2) Understanding the effects of nitric and oxalic acid cleaning on the long term performance of the filter elements would enable better scaling and comparison.

3) An evaluation of long-term (i.e., much greater than $36 \mathrm{hrs}$ ) filter flux dynamics is recommended to assess their potential impacts on scaling of filtration performance. 


\subsection{Introduction}

Pacific Northwest National Laboratory (PNNL) has been tasked by Bechtel National Inc. (BNI) on the River Protection Project-Hanford Tank Waste Treatment and Immobilization Plant (RPP-WTP) project to perform research and development activities to resolve technical issues identified for the Pretreatment Facility (PTF). The Pretreatment Engineering Platform (PEP) was designed, constructed, and operated as part of a plan to respond to issue M12, "Undemonstrated Leaching Processes," of the External Flowsheet Review Team (EFRT) issue response plan. ${ }^{\text {(a) }}$ The PEP is a $1 / 4.5$-scale test platform designed to simulate the WTP pretreatment caustic leaching, oxidative leaching, ultrafiltration solids concentration, and slurry washing processes. The PEP replicates the WTP leaching processes using prototypic equipment and control strategies. The PEP also includes non-prototypic ancillary equipment to support the core processing.

Two operating scenarios are currently being evaluated for the ultrafiltration process (UFP) and leaching operations. The first scenario has caustic leaching performed in the UFP-2 ultrafiltration feed vessels (i.e., vessel UFP-VSL-T02A in the PEP; and vessels UFP-VSL-00002A and B in the WTP PTF). The second scenario has caustic leaching conducted in the UFP-1 ultrafiltration feed preparation vessels (i.e., vessels UFP-VSL-T01A and B in the PEP; vessels UFP-VSL-00001A and B in the WTP PTF).

In both scenarios, 19-M sodium hydroxide solution ( $\mathrm{NaOH}$, caustic) is added to the waste slurry in the vessels to leach solid aluminum compounds (e.g., gibbsite, boehmite). Caustic addition is followed by a heating step that uses direct injection of steam to accelerate the leach process. Following the caustic leach, the vessel contents are cooled using vessel cooling jackets and/or external heat exchangers. The main difference between the two scenarios is that for leaching in UFP1, the 19-M NaOH is added to unconcentrated waste slurry (3-8 wt \% solids), while for leaching in UFP2, the slurry is concentrated to nominally $20 \mathrm{wt} \%$ solids using cross-flow ultrafiltration before the addition of caustic.

The PEP testing program was conducted under Test Plan TP-RPP-WTP-506 ${ }^{(\mathrm{b})}$ using a waste simulant, which was developed in response to Task 5 from the M-12 EFRT issue response plan. ${ }^{(a)}$ The testing included the following tests with simulated Hanford tank waste:

- Shakedown/Functional Testing: Tested process operations (e.g., slurry transfers, steam heating of the vessels and the accumulation of condensate, filter backpulsing, and flushing), process controls (e.g., transmembrane pressure (TMP) and axial flow velocity in the filter loop), certain test functions (e.g., in-line slurry sampling accuracy and precision).

- Integrated Test A: Demonstrated integrated processing when caustic leaching $\left(98^{\circ} \mathrm{C}\right)$ is performed in UFP-VSL-00001A/B with the $\mathrm{Cr}$ simulant component added after the post caustic leach washing step.

- Integrated Test B: Demonstrated integrated processing when the caustic leaching $\left(98^{\circ} \mathrm{C}\right)$ is performed in UFP-VSL-00002A with the Cr simulant component added after the post caustic leach washing step.

(a) SM Barnes, and R Voke. 2006. "Issue Response Plan for Implementation of External Flowsheet Review Team (EFRT) Recommendations - M12: Undemonstrated Leaching Process." 24590-WTP-PL-ENG-06-0024 Rev. 0.

(b) GB Josephson, OP Bredt, JK Young, and DE Kurath. 2009. Pretreatment Engineering Platform (PEP) Testing (Phase I). TP-RPP-WTP-506, Rev. 0.4, Pacific Northwest National Laboratory, Richland, Washington. 
- Integrated Test D: Demonstrated integrated processing when the caustic leaching is performed at a lower temperature $\left(85^{\circ} \mathrm{C}\right)$ in UFP-VSL-00002A and with the $\mathrm{Cr}$ simulant component added to the initial batch of simulant.

Integrated Test $\mathrm{C}$ was deleted from the scope of the testing (ICN-TP-RPP-WTP-506_R0.2).

In partial fulfillment of the testing requirements outlined in TP-RPP-WTP-506 ${ }^{(a)}$ (see Table S.4), the filtration performance of a non-radioactive Hanford waste slurry simulant was evaluated in both a benchscale test apparatus (the Cells Unit Filter [CUF]) and the PEP. The filter flux results from each test scale were compared to evaluate filter scale-up and to provide a basis for determining filter scale-up issues.

This report describes the results of scale-up testing at PEP. Comparative filtration tests include two separate low-solids filter conditioning tests and a single high-solids dewatering test conducted in the PEP on a bench-scale filtration system designated for non-radioactive simulant materials (i.e., the cold CUF at the Applied Process Engineering Laboratory [APEL]). The low-solids conditioning tests were conducted with an unmodified, low-solids simulant slurry feed; whereas; the high-solids dewatering test was conducted using a high-solids concentration leached and washed simulant slurry. The results of these tests are compared to support the development of a scale factor for use in the WTP.

(a) GB Josephson, OP Bredt, JK Young, and DE Kurath. 2009. Test Plan for Pretreatment Engineering Platform (PEP) Testing (Phase I). TP-RPP-WTP-506, Rev. 0.4, Pacific Northwest National Laboratory, Richland, Washington. 


\subsection{Quality Assurance}

The PNNL Quality Assurance Program is based upon the requirements defined in U.S. Department of Energy (DOE) Order 414.1C, Quality Assurance, and 10 CFR 830, Energy/Nuclear Safety Management, Subpart A-Quality Assurance Requirements (a.k.a. the Quality Rule). PNNL has chosen to implement the following consensus standards in a graded approach:

- ASME NQA-1-2000, Quality Assurance Requirements for Nuclear Facility Applications, Part 1, Requirements for Quality Assurance Programs for Nuclear Facilities.

- ASME NQA-1-2000, Part II, Subpart 2.7, Quality Assurance Requirements for Computer Software for Nuclear Facility Applications.

- ASME NQA-1-2000, Part IV, Subpart 4.2, Graded Approach Application of Quality Assurance Requirements for Research and Development.

The procedures necessary to implement the requirements are documented in PNNL's Standards-Based Management System (SBMS). The RPP-WTP quality requirements are implemented by performing work in accordance with the River Protection Project - Waste Treatment Plant Support Program (RPP-WTP) Quality Assurance Plan (RPP-WTP-QA-001, QAP). Work was performed to the quality requirements of NQA-1-1989 Part I, Basic and Supplementary Requirements, NQA-2a-1990, Part 2.7, and DOE/RW0333P, Rev 13, Quality Assurance Requirements and Descriptions (QARD), as applicable. These quality requirements are implemented through the River Protection Project - Waste Treatment Plant Support Program (RPP-WTP) Quality Assurance Manual (RPP-WTP-QA-003, QAM). The requirements of DOE/RW-0333P Rev 13, Quality Assurance Requirements and Descriptions (QARD), and 10 CFR 830 Subpart A were not required for this work.

The RPP-WTP addresses internal verification and validation activities by conducting an independent technical review of the final data report in accordance with RPP-WTP's procedure QA-RPP-WTP-604. This review procedure is part of PNNL's RPP-WTP Quality Assurance Manual (RPP-WTP-QA-003). Following this procedure, a technical review would verify that the reported results are traceable, inferences and conclusions are soundly based, and the reported work satisfies the objectives.

PEP filtration testing to support the scale-up analysis presented in the current report was impacted by three technical issues: 1) improper wiring of a process flow sensor, 2) process flow sensors subject to increased uncertainty over that listed by the manufacturer, and 3) potential stagnation of fluid in process thermowell. These issues are detailed in NCR 41090.1, NCR 38767.1, and NCR 42402.1, respectively. With regard to issue \#1, the issue was circumvented during testing by reading the process flow sensor output from the digital display on the sensor. The sensor was rewired properly at a later date. The process sensor impacted by issue \#2 was used "as-is," with the increased uncertainty noted. Finally, the thermowell issue (i.e., issue \#3) was unfixable. 



\subsection{Process and Equipment Description}

The cold-CUF and PEP test systems are designed to simulate WTP waste pre-treatment operations. Pre-treatment activities involve separation of high-level waste (HLW) solids from the low-activity waste (LAW) liquid stream by cross-flow filtration in the PTF. The waste solids intended for the HLW stream will undergo caustic and oxidative leaching processes to dissolve and wash out materials that would otherwise limit the HLW loading in the immobilized waste glass. The concentrated HLW solids are caustic leached and oxidative leached during pretreatment. After leaching steps, the HLW solids are subjected to further concentration and/or washing operations using cross-flow filtration. Although the cold-CUF and PEP test systems are designed to allow study of these pretreatment operations, these two systems differ with regard to the range of operations that can be carried out in each:

- PEP Test System: The PEP test system is designed to perform engineering-scale demonstrations of the WTP pretreatment ultrafiltration and leaching processes. Figure 3.1 presents a simplified process diagram showing the vessels, pumps, heat exchangers, and filter systems associated with the PEP. Equipment that has been considered critical for evaluating the integrated system performance has been scaled to be prototypic. Specifically, vessels UFP-VSL-T01A, -T01B, and -T02A have been scaled to be geometrically similar to the WTP with the working heights and diameters scaled by $1 / 4.5$. Pipe sizes are scaled to have approximately $1 / 4.5$ the diameter, but the fluid velocity is to be approximately the same as the full-scale plant. It should be noted that a limited subset of the process equipment shown in Figure 3.1 is used for cross-flow filtration. Those PEP systems relevant to evaluation of filtration scale-up performance are described in detail in Section 3.2.

- Cold-CUF Test System: The cold-CUF test system is designed to perform bench-scale demonstrations of select WTP pretreatment operations. The operations that can be examined on the CUF nominally include waste filtration, filter cleaning, waste solids chemical leaching, and waste solids washing. Unlike the PEP test system, CUF equipment and vessel dimensions were not designed as prototypes of WTP process equipment. In addition, all CUF equipment and instrumentation (as they currently exist) are tied to the filtration process. For example, the CUF leaching vessel also serves as the slurry reservoir for waste filtration operations. A full description of cold-CUF equipment and instrumentation is given in Section 3.3.

To facilitate comparable filtration performance on test scales, the PEP and cold-CUF test systems both employ 1) the same type of filter elements in similar cross-flow ultrafiltration configurations and 2) similar slurry mass to filter surface area ratio. Additional information regarding filter elements is provided in Section 3.1. The ratio of slurry volume/mass to filter surface area is an important parameter for filtration; in any scale-up study, it is optimal to maintain this ratio between the two different test scales (as it can impact filter transience dynamics and filtration steady state). 


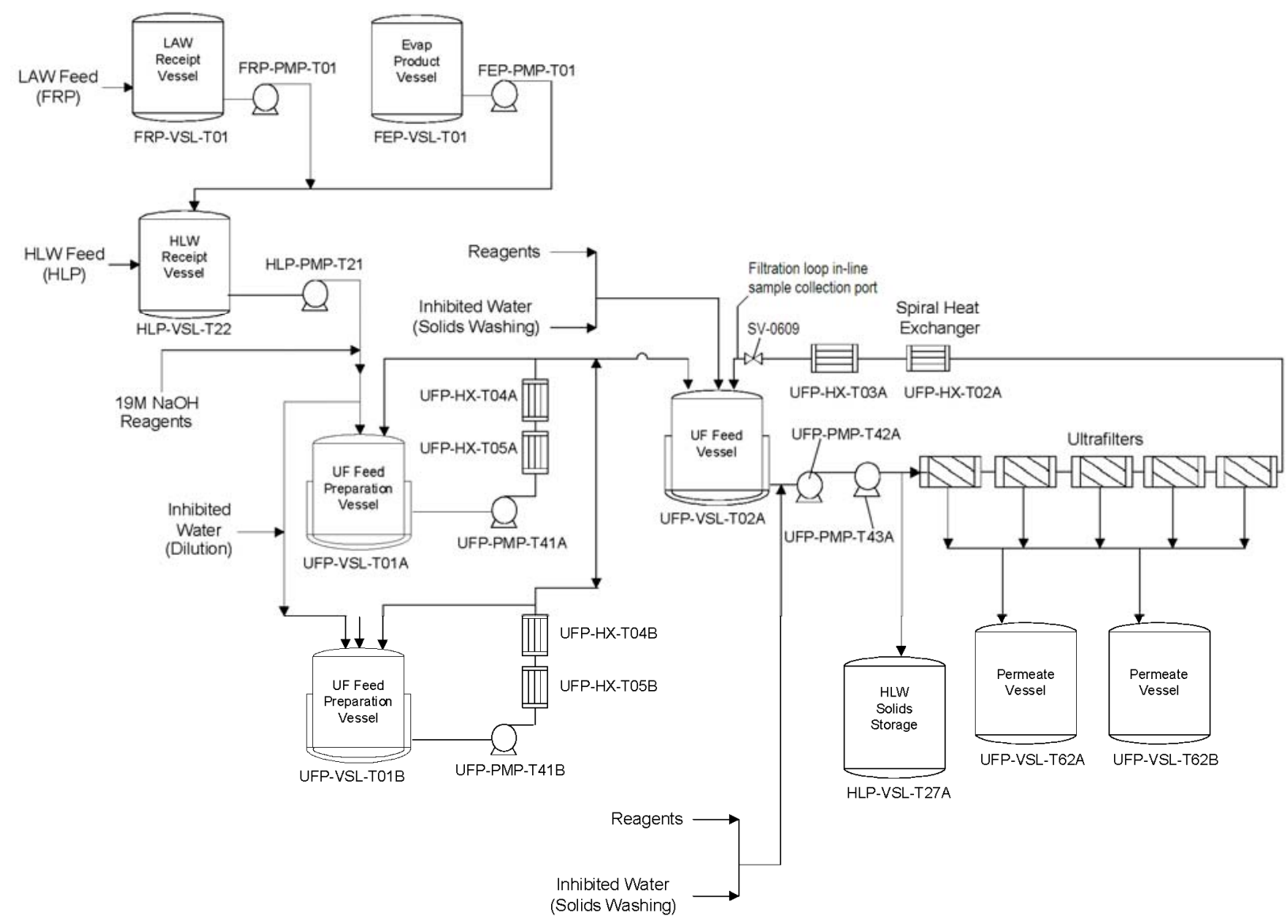

Figure 3.1. PEP Simplified Flow Diagram

\subsection{Filter Elements}

The filter elements used in PEP and CUF are porous sintered metal tubes. The filter feed flows through the inside of the filter element axially while the feed permeate passes through the tube walls radially. Filtration occurs when the pressure differential between the inside and outside walls of the filter element (the TMP) is high enough to drive the slurry permeate through the tubular walls. The axial flow across the filter walls minimizes solid buildup and allows filtration to occur continuously with minimal downtime for backpulsing to remove the solids buildup.

The filters purchased for both the PEP and cold-CUF testing were obtained from the Mott Corporation (Farmington, CT) using the same specifications as the filters being purchased for the WTP PTF. Filters for CUF and PEP were taken from the same manufacturer's lot number (see Specification WTP- $070110^{(\mathrm{a})}$ for more details). The filters are constructed of porous sintered 316 stainless steel and have a pore size of $0.1 \mu \mathrm{m}$ (as determined by bubble-point measurements). The cold-CUF employs a single 2-ft-long element (dimensions of a 2-ft element are shown in Figure 3.2). The PEP test system employs a combination of 8-ft-long and 10-ft-long filter elements, which were formed by welding either four or five $2 \mathrm{ft}$ filter elements together. As such, the PEP elements have the same radial dimensions and

(a) Specification WTP-070110, written by JGH Geeting, for PNNL Purchase Order 38825, February 2, 2007. 
filtration ratings as the 2-ft elements, but have a longer filtration length of either 96 in. or 120 in. As noted in TP-WTP-RPP-506 ${ }^{\text {(a) }}$, selection of PEP filter geometry and configuration was based on the EFRT recommendation for the integrated test platform that 1) "ultrafilter elements must be prototypic length and diameter to obtain expected filter performance data" and that 2) "the test equipment should be scaled down by using fewer filter elements in each assembly."
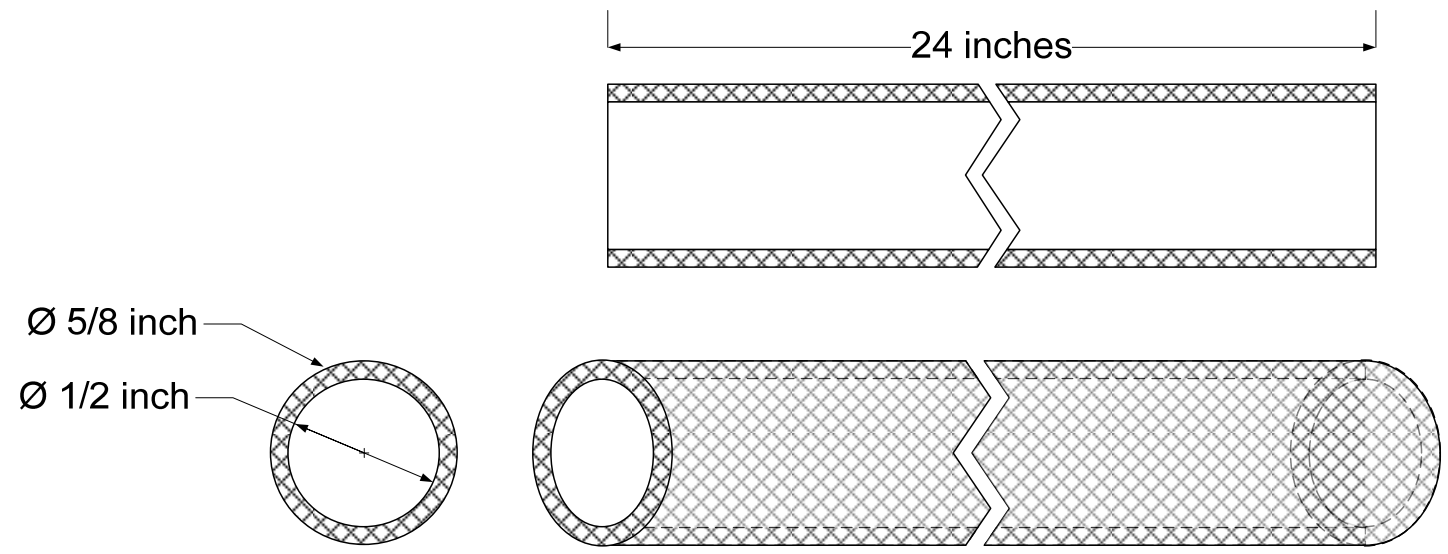

Figure 3.2. CUF Filter Element

\subsection{PEP Filtration System}

The PEP filtration system is composed of an ultrafiltration feed tank (UFP-VSL-T02A, henceforth Tank T02A), a slurry circulation and filtration loop, a permeate metering and collection system, and a filter backpulse and cleaning system. The PEP filtration system is configured to measure the feed flowrate, temperatures, and axial and TMP drop across each filter bundle. In addition, the system is configurable such that filter bundles 1-5 may be connected in series to the slurry circulation loop or bypassed such that flow is directly through filter bundle 1 or through filter bundles 2-5. In the following paragraphs, the key process equipment for slurry filtration operations are identified and discussed. A list of full PEP process equipment and instrumentation may be found in TP-RPP-WTP-506. ${ }^{\text {(a) }}$

\section{$\underline{\text { Ultrafiltration Feed Tank }}$}

Vessel UFP-VSL-T02A (hereafter referred to as Tank T02A) serves as a primary supply and mixing reservoir for slurry being circulated through the filtration loop. The contents of this tank are mixed using an array of six pulse jet mixers (PJMs). The PJMs are dimensionally scaled copies of the PTF PJMs and are located prototypically within the vessel (24590-PTF-3YD-UFP-00002). ${ }^{(b)}$ When Tank T02A contains a Newtonian fluid (e.g., during the low-solids filter flux test), they are operated to match the power/volume ratio of the PTF. When the fluid is non-Newtonian (e.g., during the high-solids filter flux test) they are operated to match the jet velocity of the PTF. Jet mixing is also introduced by the filterloop return nozzle, which is prototypically sized and located. Additional mixing within Tank T02A is

(a) GB Josephson, OP Bredt, JK Young, and DE Kurath. 2009. Pretreatment Engineering Platform (PEP) Testing (Phase I). TP-RPP-WTP-506, Rev. 0.4, Pacific Northwest National Laboratory, Richland, Washington.

(b) B Stiver. 2007. Functional Requirements for Pretreatment Engineering Platform (PEP). 24590-PTF-3YDUFP-00002, Rev. 1, Bechtel National Incorporated, Richland, Washington. 
provided by air sparge mixers and the steam-ring air purge, both of which were operated to match the power/volume of the PTF (TP-RPP-WTP-506). ${ }^{\text {(a) }}$ Ancillary systems for Tank T02A include bubblers to measure slurry density and level, laser level sensors, and an array of resistance temperature detectors (RTDs) to measure the tank temperature profile. Tank T02A is equipped with a water jacket supplied with chilled water to cool the contained slurry.

Slurry Filtration Circulation Loop

The filtration loop contains process equipment key to slurry dewatering and washing operations. It is composed of two slurry pumps, a series of five filter bundles, and two heat exchangers (see Figure 3.1).

Two functionally prototypic centrifugal slurry pumps, UFP-PMP-T42A and UFP-PMP-T43A (hereafter referred to as T42A and T43A, respectively), are operated in series to provide the required slurry flowrate and pressure for the cross-flow filter bundles. The suction to T42A is fed by Tank T02A. In addition, the feed to pump T42A is connected to process inhibited water ${ }^{(b)}$ supplies used in slurry washing and dilution operations. The discharge from pump T42A feeds pump T43A. Slurry discharge from pump T43A can be fed through, or bypassed around, the cross-flow filter bundles. Pumps T42A and T43A provide a combined filtration loop flowrate and pressure of up to $150 \mathrm{GPM}$ and $250 \mathrm{psig}$.

The cross-flow filter system is the core of slurry liquid-solid separations. It is composed of five filter bundles operated in series, prototypic of the PTF. These filter bundles are designated as UFP-FILT-T01A to -T05A (hereafter referred to as Filters 1 through 5). Each bundle consists of 12 individual filter elements. These elements are porous sintered stainless steel tubes of 0.5 -inch inside diameter and 8- or 10-foot length. A summary of the geometries of the five filter bundles is provided in Table 3.1. In addition, Figure 3.3 and Figure 3.4 show the complete filter bundle assembly and filter element arrangement. The pipe-reducer end-caps on each filter bundle are similar to those of the PTF to provide similar entrance and exit effects.

The PEP filtration system has a total surface area of up to $72.3 \mathrm{ft}^{2}$, which is approximately 276 times greater than that of the cold-CUF. It should be noted that relative to the plant-scale (WTP) filtration operations, the PEP filter banks have approximately $1 /(4.5)^{2}$ less filtration area. The filtration area was scaled by maintaining the same number of filter bundles (and filter element length) and by reducing the number of filters in each bundle from 241 (plant scale) to 12 (PEP scale). The filter loop is equipped with slurry bypass valves to allow slurry flow through Filter Bundle 1 and/or Filters 2 through 5 . When operated with only Filter 1, the PEP matches the (Tank T02A slurry volume)/(filter surface area) ratio of the PTF. When operated with all five filters, the PEP can approximately match the (filtration rate)/(Tank T02A mixing rate) ratio of the PTF. TP-RPP-WTP-506 $6^{(a)}$ discusses the selection of operating parameters and filter-loop configurations to maximize the similarity between the PEP and PTF.

Tubeside slurry flowrate and pressure are monitored by two flow meters and a series of pressure transducers (see Section 3.4 for details). Slurry flow to pump T42A is measured by a magnetic flow meter FT-0623. Slurry discharge flow from pump T43A is measured by a second magnetic flow meter (FT-0635). Circulation loop pressure is monitored by a series of pressure transducers located at the entrance to each slurry pump, filter bundle, and heat exchanger. Target PEP TMPs and axial velocities

(a) GB Josephson, OP Bredt, JK Young, and DE Kurath. 2009. Test Plan for Pretreatment Engineering Platform (PEP) Testing (Phase I). TP-RPP-WTP-506, Rev. 0.4, Pacific Northwest National Laboratory, Richland, Washington.

(b) Inhibited water typically refers to a $0.01 \mathrm{M}$ solution of $\mathrm{NaOH}$. 
(AVs) were to match those of the PTF to maximize the similarity of performance between the PEP and PTF.

The slurry filtration loop also includes two in-line heat exchangers that are available for temperature control of Tank T02A and/or the slurry filtration loop. The first heat exchanger, UFP-HX-T02A, is a dimensionally prototypic spiral plate heat exchanger that uses chilled water to cool the circulating slurry. This heat exchanger was typically used to remove mechanical heat input to the slurry by the pumps T42A and T43A. The second heat exchanger, UFP-HX-T03A, is a steam exchanger intended to heat the slurry (if needed for leaching operations) and is not prototypic of the PTF. For the current testing, UFP-HXT03A was not used. Both heat exchangers are equipped with a bypass loop so that they can be isolated from slurry flow. Heat exchanger performance is monitored and controlled with RTDs installed in thermowells (see Section 3.4 for details). The final process element in the slurry circulation loop is a pressure control valve (SV-0609), which can be adjusted in combination with the slurry pumps to provide adequate backpressure for permeate production. After passing through SV-0609, the dewatered circulating slurry is recycled back into Tank T02A.

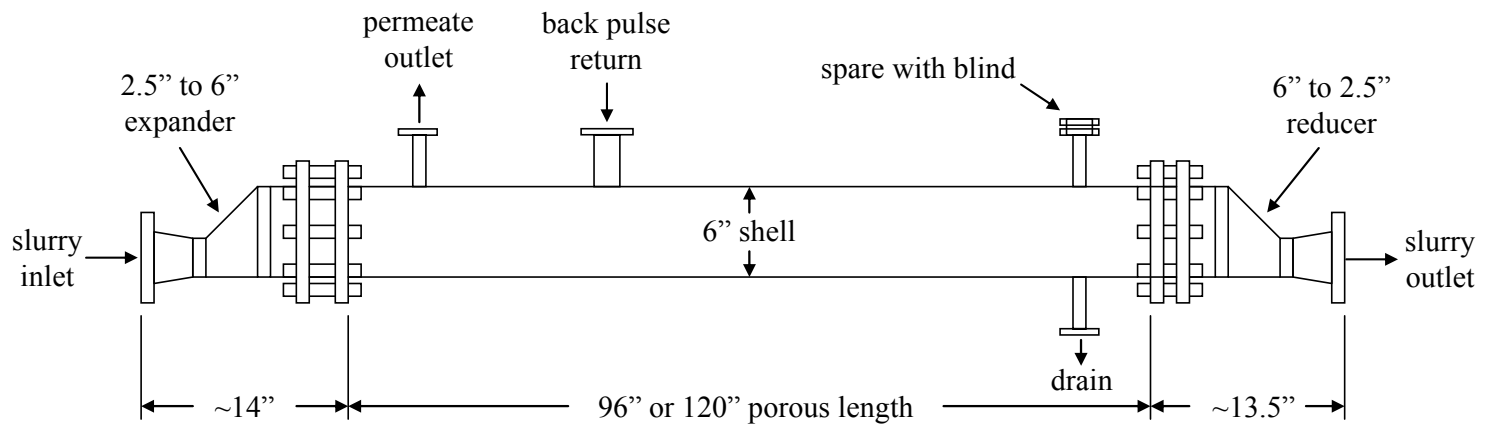

Figure 3.3. PEP Filter Bundle Assembly with Key Geometric Parameters Listed (drawing not to scale)

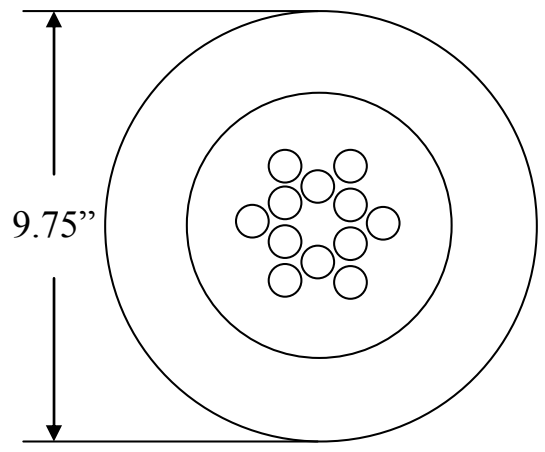

AXIAL VIEW
Twelve 96"/120" long, 0.5"

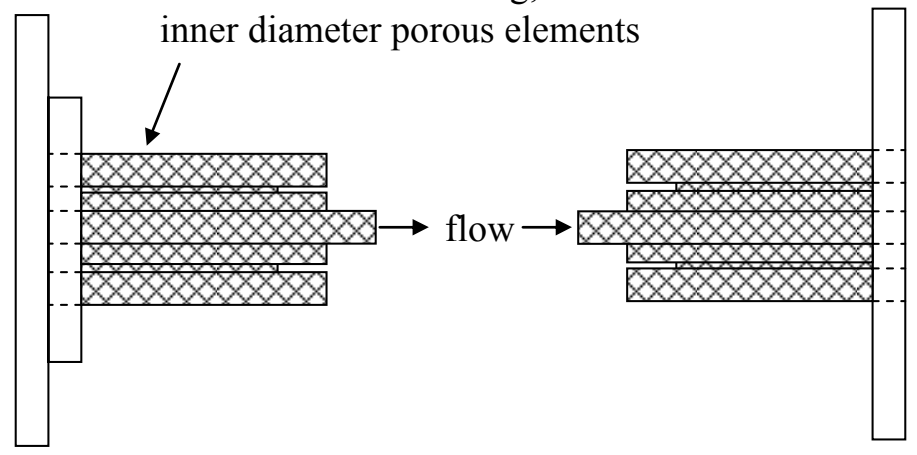

SIDE VIEW

Figure 3.4. PEP Filter Element Arrangement (axial and side views—drawings not to scale) 
Table 3.1. Specifications of the Five PEP Cross-Flow Filtration Bundles

\begin{tabular}{cccccc}
\hline Filter \# & Filter ID & $\begin{array}{c}\text { Number of } \\
\text { Elements in } \\
\text { Bundle }\end{array}$ & $\begin{array}{c}\text { Element } \\
\text { Inside } \\
\text { Diameter } \\
{[\text { inches }]}\end{array}$ & $\begin{array}{c}\text { Element } \\
\text { Length [ft] }\end{array}$ & $\begin{array}{c}\text { Bundle } \\
\text { Surface Area } \\
{\left[\mathrm{ft}^{2}\right]}\end{array}$ \\
\hline 1 & UFP-FILT-T01A & 12 & 0.5 & 10 & 15.7 \\
2 & UFP-FILT-T02A & 12 & 0.5 & 10 & 15.7 \\
3 & UFP-FILT-T03A & 12 & 0.5 & 10 & 15.7 \\
4 & UFP-FILT-T04A & 12 & 0.5 & 8 & 12.6 \\
5 & UFP-FILT-T05A & 12 & 0.5 & 8 & 12.6 \\
Total & n/a & n/a & n/a & n/a & 72.3 \\
\hline
\end{tabular}

$\underline{\text { Permeate Metering and Collection Systems/Filtration Backpulse Systems }}$

The permeate metering and collection systems consist of Coriolis mass flow meters for monitoring permeate production rates, permeate collection tanks, and three pulse pots connected to high pressure air supplies for backpulsing the filter bundles.

Permeate (shell-side) mass production rates from filters 1 through 5 are monitored by Coriolis flow meters. Permeate flow from each of the filter bundles is directed to three pulse pots (designated as UFPPP-T01A to UFP-PP-T03A). Similar to the PTF, pulse pot UFP-PP-T03A serves filter bundle 1, pulse pot UFP-PP-T02A serves filter bundles 2 and 4, and pulse pot UFP-PP-T01A serves filter bundles 3 and 5. They are also operated in the PEP in the same way as they would be in the PTF. The pulse pots are filled with a sufficient volume of collected permeate to backpulse the filter bundles. Overflow from the pulse pots may be directed to 1) permeate or process slurry collection tanks (UFP-VSL-T62A and T62B) during slurry dewatering operations or 2) a return line to Tank T02A during continuous recycle filtration operations. A summary of the permeate metering and pulse pot systems is provided in Table 3.2. A simplified schematic of pulse-pot to filter and collection tank flow connections is shown in Figure 3.5 .

Table 3.2. Permeate Metering and Pulse Pot Configurations for PEP

\begin{tabular}{lll}
\hline Filter Bundle No. / ID & $\begin{array}{l}\text { Permeate } \\
\text { Coriolis Meter }\end{array}$ & $\begin{array}{l}\text { Associated Pulse } \\
\text { Pot }\end{array}$ \\
\hline 1-UFP-FILT-T01A & FT-0720 & UFP-PP-T03A \\
2-UFP-FILT-T02A & FT-0755 & UFP-PP-T02A \\
3-UFP-FILT-T03A & FT-0765 & UFP-PP-T01A \\
4- UFP-FILT-T04A & FT-0775 & UFP-PP-T02A \\
5-UFP-FILT-T05A & FT-0785 & UFP-PP-T01A \\
\hline
\end{tabular}




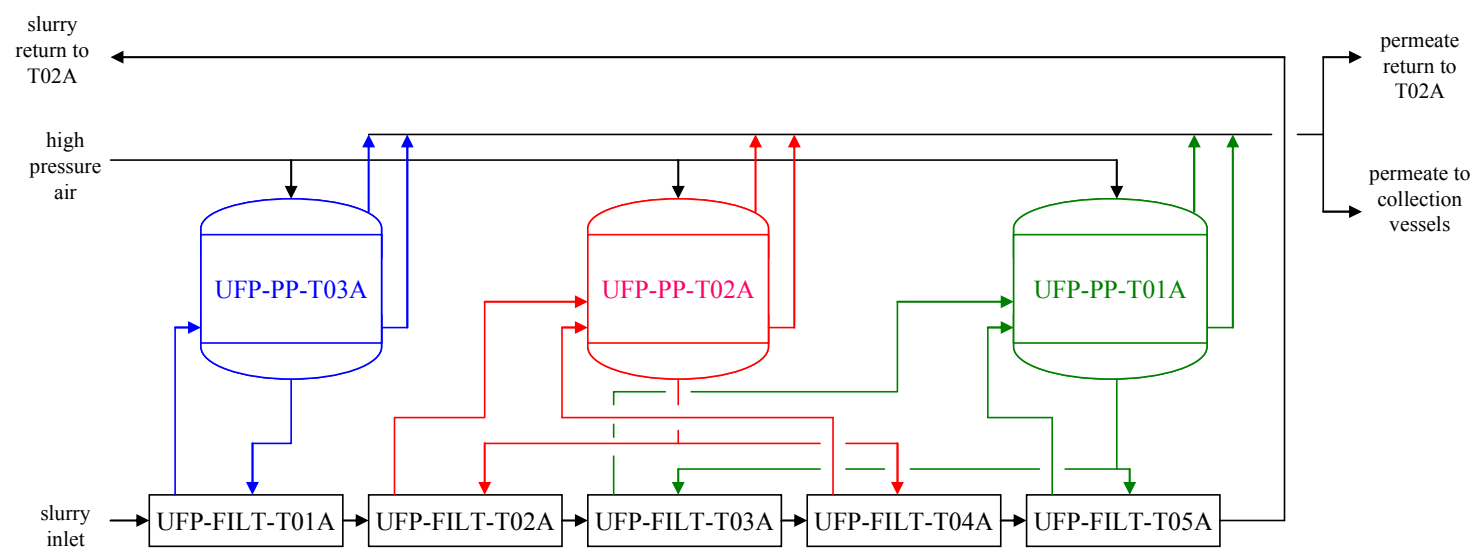

Figure 3.5. Simplified Schematic of the Flow Connections for the PEP Permeate Metering and Collection Systems

The pulse pots are for backpulsing the filter bundles. During backpulsing, one of the pulse pots is isolated and charged with high-pressure air until the pressure reaches about $100 \mathrm{psig}$. The outlet near the middle of the pot is open, and the pulse pot level is decreased to a specified level ( $\sim 9$ inches). The outlet valve is closed. The level is adjusted so that the backpulse will provide a consistent volume without blowing air through the filters. The pulse pot is repressurized until the pulse pot pressure exceeds the tubeside pressure of the filter bundle to be backpulsed by a given amount (typically 40 psid). After the target pulse pot pressure is reached, the fast-acting valve isolating the pulse pot from the filter is opened, and the permeate left in the pulse pot flows back through filter element until a lower pressure differential is reached. The lower pressure shutoff was typically set at 5 psig. Backflow of permeate forces loosen any particles that are weakly entrained in the filter pores or that have caked on the filter surface.

The backpulsing function of the filter loop can be operated only when actively filtering T02A contents. There are three variables that can be set by the operator:

1) The "Level Drain Set Point"- the height of fluid in the pulse pot used for the backpulse

2) The "Backpulse Pressure Set Point"- the amount above the filter inlet pressure that the pulse pot should be charged to (i.e., if the inlet pressure is 100 psig, and the Backpulse Pressure Set Point is set to $40 \mathrm{psig}$, the control system will charge the pulse pot to $140 \mathrm{psig}$ )

3) The "Pressure Deadband for Completion"- - the amount above the filter inlet pressure that will cause the backpulse to be marked as finished (i.e., if the inlet pressure is $100 \mathrm{psig}$, and the Deadband is set to $10 \mathrm{psig}$, the backpulse will complete when the pulse pot pressure indicator gets down to $110 \mathrm{psig}$ ).

During filtering, the operator initiates the backpulse cycle through the PEP Human Machine Interface (HMI). For a "typical" backpulse cycle, the first step is to close all valves entering and leaving the pulse pot. Next, the high-pressure air line is opened, and the pulse pot is pressurized to $50 \mathrm{psig}$. The highpressure air valve is closed, and the drain valve to T62A/B is opened. The pulse pot fluid level falls until reaching the Level Drain Set Point when the drain valve is closed. The high-pressure air valve is opened again and pressurizes the pulse pot to the sum of the filter inlet pressure plus the Backpulse Pressure Set Point ( $100 \mathrm{psig}+40 \mathrm{psig}=140 \mathrm{psig}$ in the above example). The air valve is closed, and the backpulse cycle pauses for 15 seconds. The fast-acting valve then opens, and the pressure in the pulse pot pushes fluid back through the filter until the pressure in the pulse pot is equal to the filter inlet pressure plus the 
deadband ( $100 \mathrm{psig}+10 \mathrm{psig}=110 \mathrm{psig}$ in the above example). The final step is to return to filtering conditions. The fast-acting valve closes, and the filter outlet valve and pulse pot outlet valve to T62A/B (not the drain valve) are opened.

During PEP processes, slurry samples are collected using either an in-tank sampler (to collect samples directly from Tank T02A) or in-line sampler (to collect filtration loop samples). In-line samples were obtained from the slurry recirculation loop by drawing a side stream from the process flow as shown in Figure 3.6. To obtain a sample, the second valve was fully opened and then the first valve was opened sufficiently to allow samples to be safely obtained. The sample line and valves were purged with at least 3 line volumes before each sampling event. For the current report, all PEP in-line samples were taken from the filtration loop after the filtration back pressure control valve (i.e., SV-0609 - see Figure 3.1). The in-tank sampling system for T02A is shown in Figure 3.7. The sample collection port for in-tank samples for T02A is located immediately following the sample loop recirculation pump SAM-PMPT02A. Tank T02 "in-tank" samples were obtained with the sample loop in recirculation mode with slurry returned to the vessel. To obtain a sample, a valve was used to divert the entire flow to the sample bottle. The sampling valve and line was purged before each sample to ensure that there was no cross contamination with previous sampling events. Samples for this test were taken at the lowest height at the middle position, 15.1 inch from the center ( $81 \%$ of total radius) and 2 inches from the bottom.

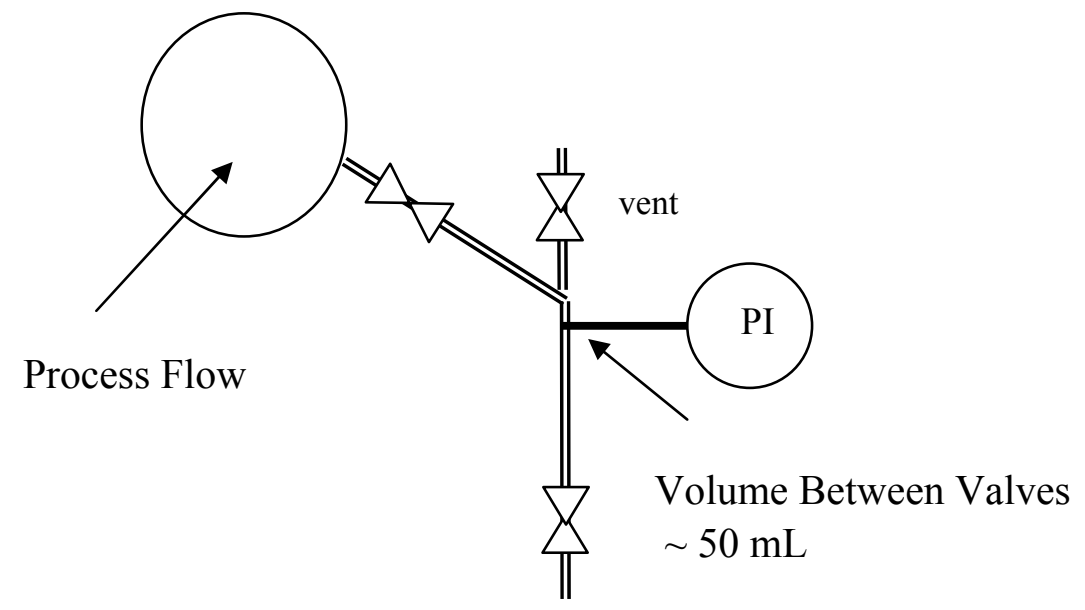

Figure 3.6. Simple In-Line Sample Valving 


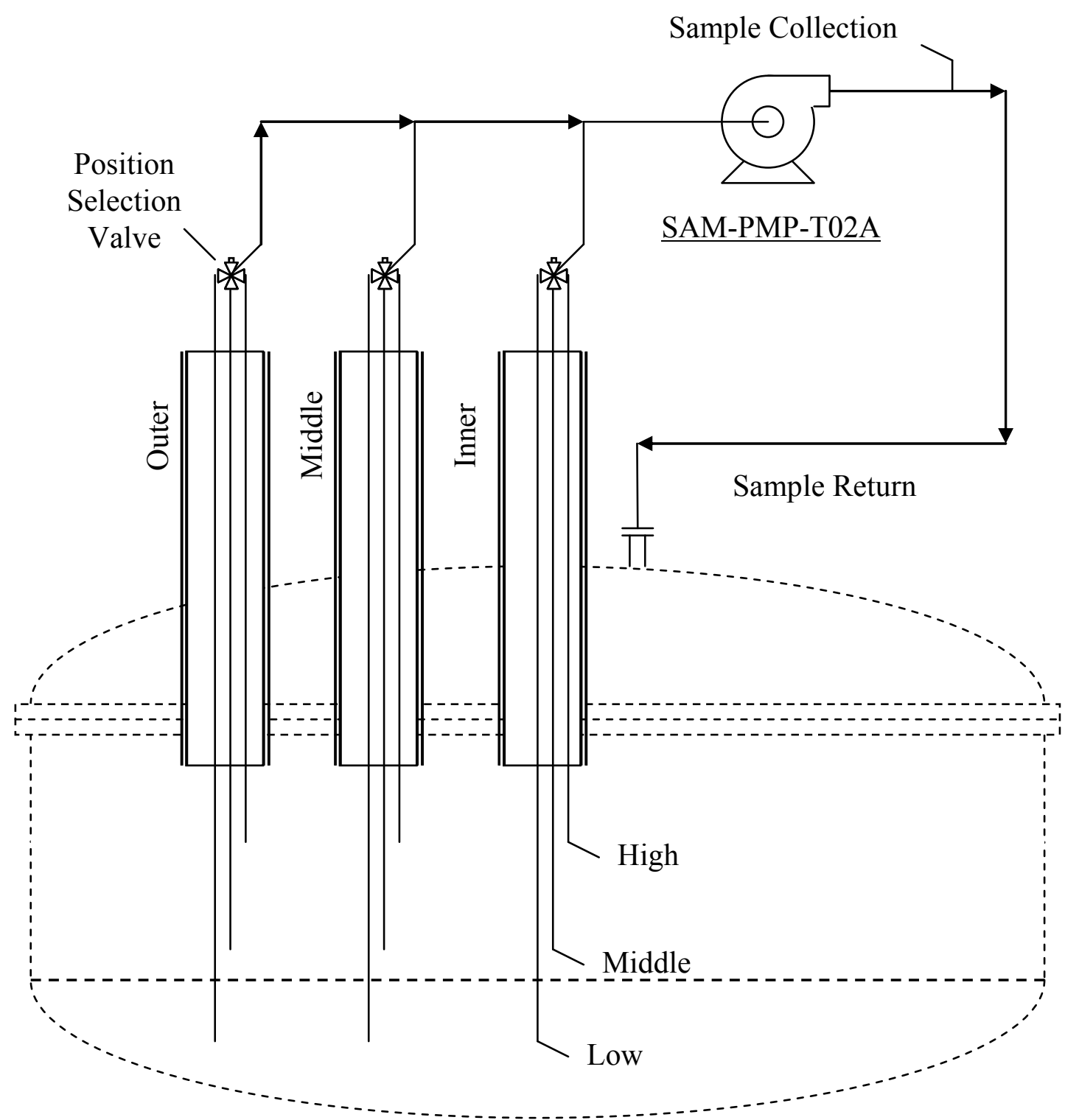

Figure 3.7. In-Tank Sample Collection System for Tank T02A Showing the Three Radial Positions at Three Heights and Sampling Flow Loop

\subsection{Cold-CUF Filtration System}

The cold-CUF filtration system is composed of five main components: 1) a slurry reservoir tank, 2) a slurry recirculation loop, 3) a CUF filter assembly, 4) a permeate flow loop, and 5) a permeate backpulse chamber. Figure 3.8 shows a piping diagram of the CUF. Figure 3.9 is a photograph of the assembled testing apparatus. The 3-HP electric motor and positive displacement pump that drives the filtration slurry simulant are shown to the left in this view. 


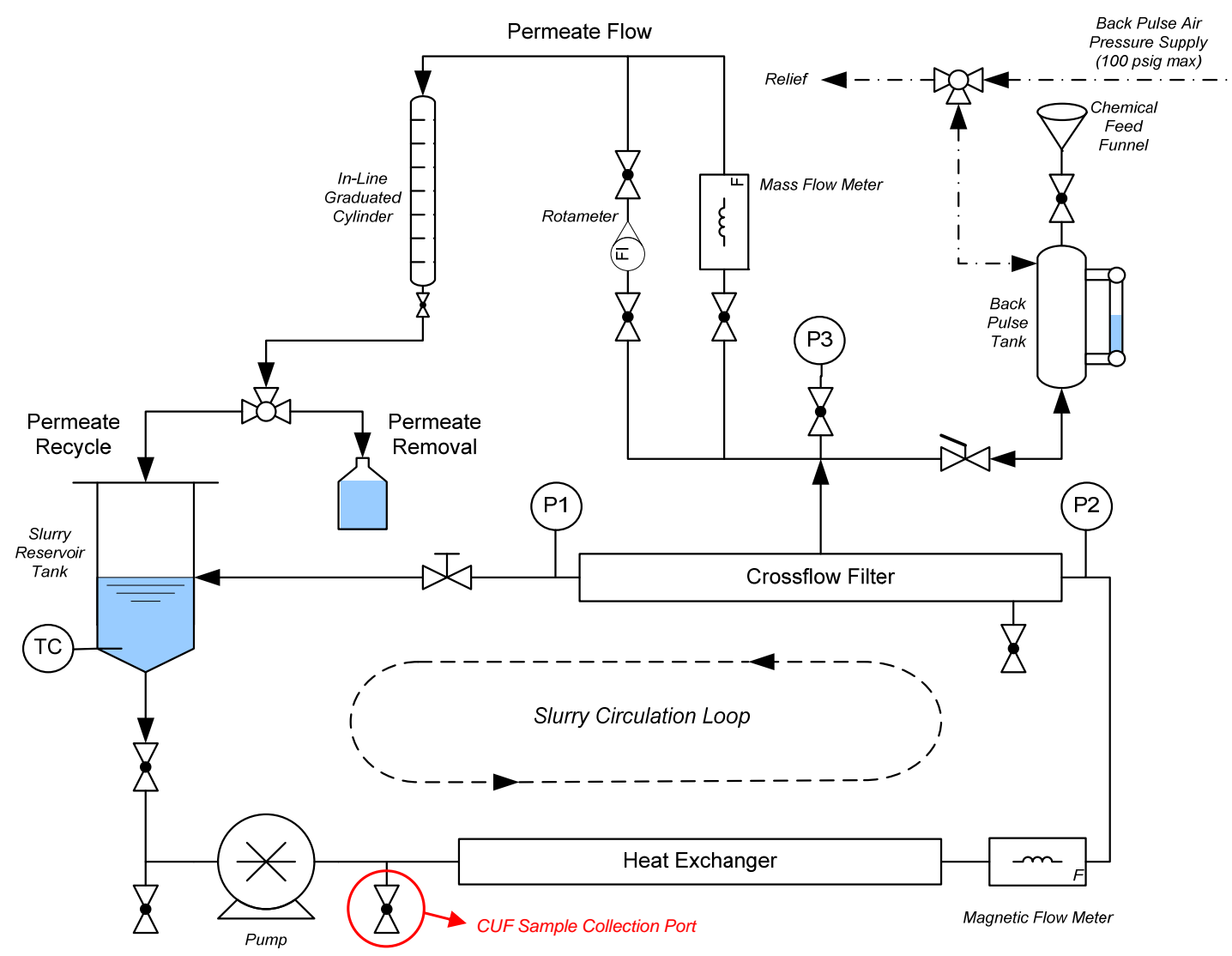

Figure 3.8. CUF Piping Diagram

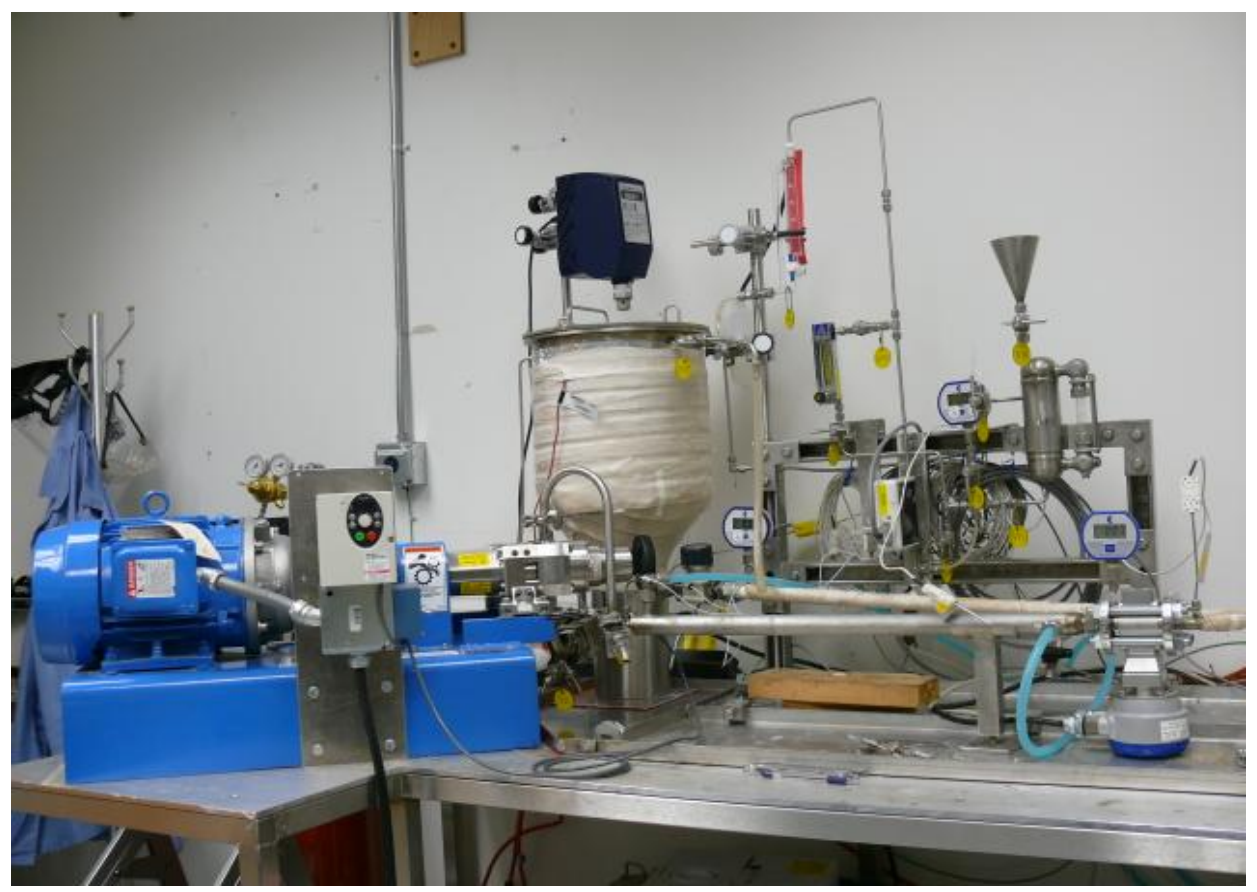

Figure 3.9. The Cold-CUF Apparatus 
The slurry reservoir tank is a $25-\mathrm{L}$ tank constructed of 304-L stainless steel. It is composed of two cylindrical sections of 5-in and 12-in inner diameter with a conical transition section between them.

Figure 3.9 shows the upper and conical parts of the reservoir (the lower cylinder resides behind the pump housing). All sections are appropriately baffled with four baffles in the 12-in diameter section and transition section and three baffles in the 5-in diameter section. Agitation in the tank is provided from an overhead mixer using two impellers: 1) 2-inch-diameter, 3-blade marine propeller at the end of the shaft at one tank radius from the bottom and 2) 3-inch-diameter, pitched, 3-blade turbine positioned 5 inches above the propeller. Both impellers push fluid toward the suction line to the pump. To facilitate draining, the bottom of the vessel is sloped at a $15^{\circ}$ angle. The slurry reservoir thermocouple is installed near the bottom of the tank, extending just below the overhead mixing impeller.

In the slurry recirculation loop, a progressive cavity rotary-lobe pump directs slurry flow from the slurry reservoir through the heat exchanger, magnetic flow sensor, filter element, and back into the slurry reservoir. The bottom of the slurry reservoir is connected to the suction side of the slurry pump and the discharge of the pump first flows through a single-pass shell-and-tube heat exchanger used to remove excess heat from mechanical energy input and heat generated from frictional flow. Next, the slurry flows through a magnetic flow sensor that monitors the volumetric flow of the slurry inside the slurry recirculation loop. The data from this device are used to calculate the AV inside the filter element.

The flowing slurry then enters the CUF filter assembly. All cold-CUF tests used a single 2-ft-long filter element of 0.5-inch inner diameter and having an effective filtration area of $0.262 \mathrm{ft}^{2}$. This element was received from Mott installed in a tube-in-tube configuration. In this configuration, the outer tube surrounding the filter element has been added to capture the filtrate. The outer tube has two stainless steel tubes exiting from the filter assembly, one in the center to collect filtrate from the filter, and the other near the inlet of the filter to function as a drain. Pressure gauge ports are installed on the inlet and outlet connections to the assembly to measure the pressure inside the filter (P1 and P2 in Figure 3.8). Figure 3.10 and Figure 3.11 show the filter element assembly used in cold-CUF testing.

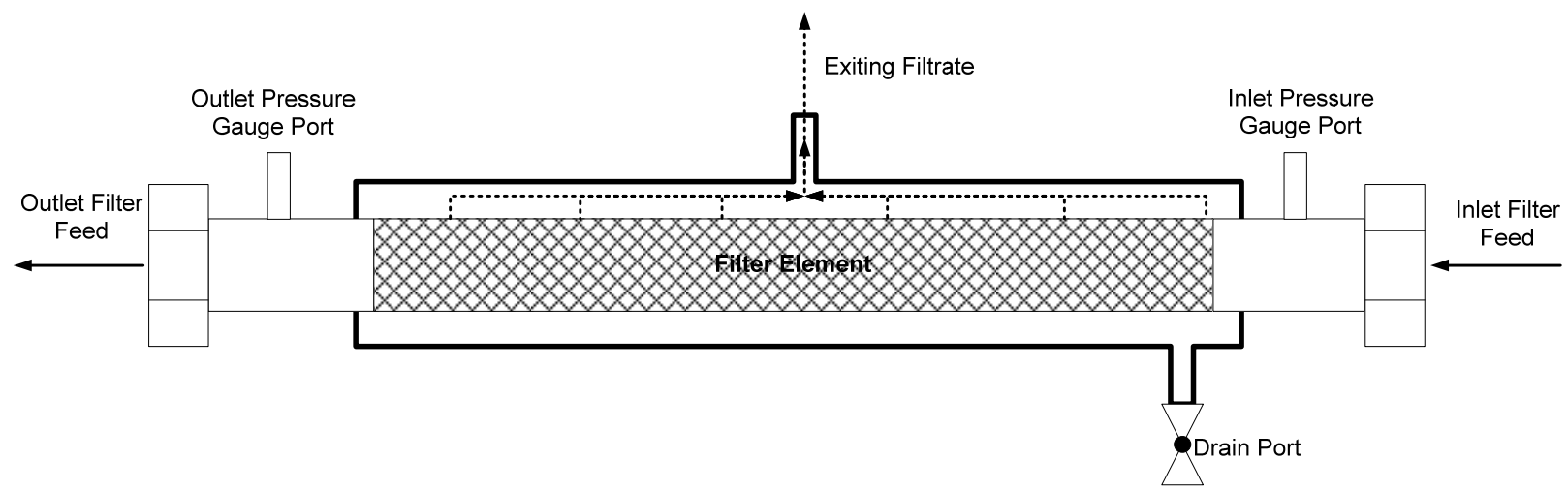

Figure 3.10. CUF Filter Assembly Sketch (not to scale) 


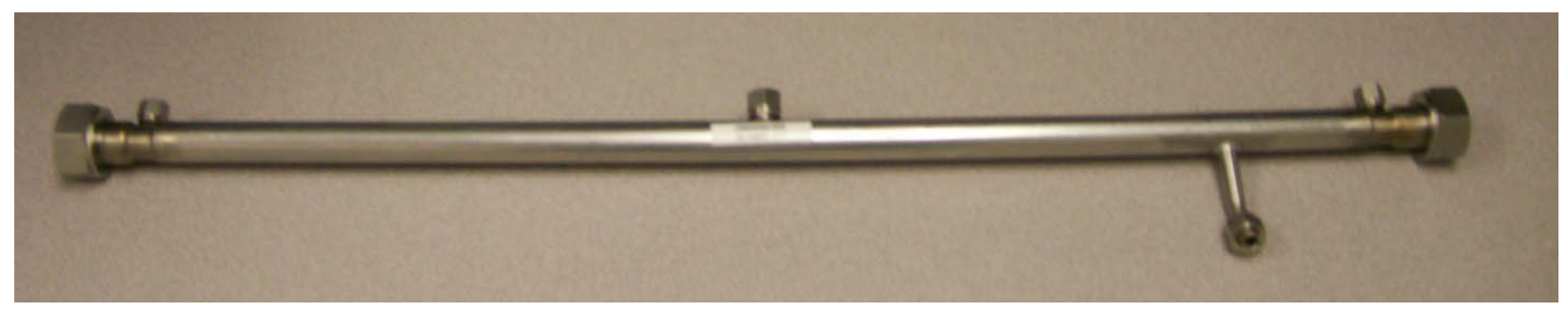

Figure 3.11. The Cell Unit Filter Assembly

Digital pressure gauges are installed on the inlet and outlet port of the filter displaying the pressure at both locations in pounds-per-square-inch gauge (psig). The data from these devices were used to calculate the average pressure inside the filter and the axial pressure drop across the element.

A manual pinch valve is located at the filter's discharge. The valve is used to adjust the pressure inside the filter to drive permeate flow through the filter membrane wall. The downstream side of this valve is connected to the slurry reservoir tank, completing the slurry recirculation loop.

The permeate flow loop starts at the center of the filter assembly where the permeate from the outer tube of the filter assembly is directed through a series of measurement devices. A digital pressure gauge at this point measures the pressure on the permeate side of the filter in psig (P3 in Figure 3.8). The TMP across the filter is calculated by subtracting the pressure on the permeate side of the filter from the average pressure of the inlet and outlet tube-side pressures. The TMP is reported in pounds-per squareinch differential pressure (psid).

Permeate flow is directed through one of two mass flow meters connected in parallel, one calibrated up to $0.18 \mathrm{~L} / \mathrm{min}$ and the other calibrated up to $1.2 \mathrm{~L} / \mathrm{min}$. The mass flow meters also measure the density of the permeate flow. An in-line graduated glass cylinder installed after the meters is used to take manual measurements of the permeate flowrate. Following these measurement devices, the permeate exits through a three-way valve. This valve may be oriented to direct permeate back to the slurry reservoir tank to be mixed with the slurry (known as "recycle mode") or to a sampling hose used to collect permeate into sample containers.

The permeate backpulse chamber is located to the right of the permeate flow loop and connected to the filter at the same location of the permeate pressure gauge. The chamber is a stainless steel vessel of approximately $425-\mathrm{mL}$ with a sight-glass to track the permeate volume inside the chamber. The chamber has three entry ports:

- A $1 / 4$-in. line with a two-way valve on the bottom connecting the vessel to the permeate side of the filter

- $\mathrm{A}^{3} / 8$-in. line with a two-way valve connecting the top of the vessel to a funnel

- A $1 / 4-i n$. line with a three-way valve connecting the top of the vessel to a compressed air line and vent line connected to the top of the slurry reservoir tank.

When opened by the toggle valve, the bottom line is used to direct permeate flow from the chamber to the filter. The funnel on the top of the chamber is used to introduce cleaning and rinse solutions directly to the vessel. The compressed gas line is used to pressurize the fluid in the chamber with compressed gas and to vent the chamber to atmospheric pressure. 
To backpulse the filter, the vessel is first vented to atmospheric pressure. Next, the toggle valve is opened to allow permeate to fill the chamber. Once the chamber is half full of permeate (as seen from the sight-glass), the valve is closed. The three-way valve is then positioned to allow compressed gas at 80 psig to fill the chamber and pressurize the fluid. The three-way valve is then positioned to isolate the pressurized chamber. Next, the slurry pressure inside the filter is decreased below 20 psig. The toggle valve at the bottom of the tank is then opened allowing the pressurized permeate inside the chamber to flow backwards through the filter element. The toggle valve is closed when the permeate level drops below the visible portion of the sight glass. After the backpulse has been applied to the filter, the threeway valve is positioned to vent the chamber back to atmospheric pressure.

Slurry samples may be taken from the system in two ways, either withdrawn from the slurry reservoir via pipette or collected from a sample valve connected to the slurry recirculation loop (shown between the pump and heat exchanger in Figure 3.8). For these tests, samples were collected from the sample valve unless otherwise noted. Before collection, 30 to $50 \mathrm{~mL}$ of slurry was discharged from the valve and was set aside. It should be noted that the discharge volume was equivalent to 3 to 5 sample leg volumes (assuming hold-up in valve to be $\sim 10 \mathrm{~mL}$ ). After the prescribed samples had been collected, the slurry volume set aside was added back to the slurry reservoir.

\subsection{Measurement and Analysis of Filtration Data}

Filtration performance for both PEP and cold-CUF test scales is assessed through process instrumentation and measurement and through analysis of slurry and permeate samples collected during testing. Each test system is equipped with an array of test instrumentation to measure process parameters, such as slurry flow, and equipment performance, such as the rate of permeate production. Postmeasurement analysis of this data is used to reduce and/or convert test results to a more usable form (e.g., converting a mass permeate production rate to a filter flux). Analytical analysis of slurry samples provides further information on process performance (such as the range of solids concentrations achieved during dewatering operations). In this section, a brief description of the measuring instrumentation for both the PEP and cold-CUF filtrations systems is given. Next, the equations used to analyze filtration results are defined. Finally, an overview of the analytical techniques used to characterize slurry and permeate samples is given.

\subsubsection{PEP Instrumentation}

Key components for measuring filtration performance at PEP include slurry and permeate flow meters, feed tank (Tank T02A) temperature sensors, and filter-loop pressure sensors. These sensors allow assessment of process conditions driving cross-flow filtration and of the performance of the filters in terms of permeate production. The process parameters of interest are 1) filter AV (or slurry flowrate), 2) filter transmembrane and axial pressure drop, and 3) rate of permeate production. Permeate production is typically corrected for variations in slurry/supernate temperature (as well as variations in TMP), and, as such, the process temperature is also of interest.

Table 3.3 provides a summary of the PEP instrumentation used to assess slurry flowrates in the filtration loop. The two flow meters listed provide measurement of both suction and discharge flowrates from the circulation loop pumping system. Flow meter FT-0623 operates at the head pressure of the Tank T02A or lower, whereas flow meter FT-0635 operates at high pressure. Because of the pressure differential between flow meters, air entrained in the suction line is likely forced into solution on the 
discharge side. Some degree of air entrainment is expected from sparging of T02A contents and slurry return. Because magnetic flow meters are sensitive to non-conductive phases like air, the reading on the discharge magnetic flow meter (FT-0635) is typically a few percent lower than that on the suction flow meter (FT-0623) for typical air entrainments observed during PEP runs. The divergence in flow meter readings may become severe if air entrainment becomes significant, but such behavior was not observed during the assessments of filter scaling effects.

Table 3.3. PEP Instrumentation Used to Assess Slurry Flowrates Through the Filtration Circulation Loop

\begin{tabular}{clc}
\hline Instrument ID & \multicolumn{1}{c}{ Description } & Units \\
\hline FT-0623 & $\begin{array}{l}\text { Instrument FT-0623 is a magnetic flow meter used to measure the volumetric } \\
\text { flowrate of slurry entering pump T42A. It is located at the suction to pump }\end{array}$ \\
& $\begin{array}{l}\text { T42A. } \\
\text { Instrument FT-0635 is a magnetic flow meter used to measure the volumetric } \\
\text { FT-0635 } \\
\text { flowrate of slurry exiting pump T43A. It is located at the discharge to pump } \\
\text { T43A. }\end{array}$ \\
\end{tabular}

Determination of transmembrane and axial pressure differentials requires knowledge of the tubeside inlet/outlet pressures and of the shellside pressure for each filter bundle. Table 3.4 provides a summary of the filter bundle instrumentation that provides this capability. 
Table 3.4. PEP Filter Bundle Pressure Sensing Instrumentation

\begin{tabular}{|c|c|c|c|}
\hline Filter Bundle & Instrument ID & Description & Units \\
\hline \multirow[t]{3}{*}{1} & PT-0739 & $\begin{array}{l}\text { PT- } 0739 \text { provides measurement of the tubeside inlet pressure } \\
\text { for filter bundle } 1 \text {. It is located at the entrance to filter bundle } 1 \text {. }\end{array}$ & psig \\
\hline & PT-0749 & $\begin{array}{l}\text { PT- } 0749 \text { provides measurement of the tubeside outlet pressure } \\
\text { for filter bundle } 1 \text {. It is located at the entrance to filter bundle } 2 \text {. }\end{array}$ & psig \\
\hline & PT-0741 & $\begin{array}{l}\text { PT- } 0741 \text { provides measurement of the shellside pressure for } \\
\text { filter bundle } 1 \text {. }\end{array}$ & psig \\
\hline \multirow[t]{3}{*}{2} & PT-0749 & $\begin{array}{l}\text { PT- } 0749 \text { provides measurement of the tubeside inlet pressure } \\
\text { for filter bundle } 2 \text {. It is located at the entrance to filter bundle } 2 \text {. }\end{array}$ & psig \\
\hline & PT-0759 & $\begin{array}{l}\text { PT- } 0759 \text { provides measurement of the tubeside outlet pressure } \\
\text { for filter bundle } 2 \text {. It is located at the entrance to filter bundle } 3 \text {. }\end{array}$ & psig \\
\hline & PT-0751 & $\begin{array}{l}\text { PT- } 0751 \text { provides measurement of the shellside pressure for } \\
\text { filter bundle } 2 \text {. }\end{array}$ & psig \\
\hline \multirow[t]{3}{*}{3} & PT-0759 & $\begin{array}{l}\text { PT- } 0759 \text { provides measurement of the tubeside inlet pressure } \\
\text { for filter bundle } 3 \text {. It is located at the entrance to filter bundle } 3 \text {. }\end{array}$ & psig \\
\hline & PT-0769 & $\begin{array}{l}\text { PT- } 0769 \text { provides measurement of the tubeside outlet pressure } \\
\text { for filter bundle } 3 \text {. It is located at the entrance to filter bundle } 4 \text {. }\end{array}$ & psig \\
\hline & PT-0761 & $\begin{array}{l}\text { PT- } 0761 \text { provides measurement of the shellside pressure for } \\
\text { filter bundle } 3 \text {. }\end{array}$ & psig \\
\hline \multirow[t]{3}{*}{4} & PT-0769 & $\begin{array}{l}\text { PT- } 0769 \text { provides measurement of the tubeside inlet pressure } \\
\text { for filter bundle } 4 \text {. It is located at the entrance to filter bundle } 4 \text {. }\end{array}$ & psig \\
\hline & PT-0779 & $\begin{array}{l}\text { PT- } 0779 \text { provides measurement of the tubeside outlet pressure } \\
\text { for filter bundle } 4 \text {. It is located at the entrance to filter bundle } 5 \text {. }\end{array}$ & psig \\
\hline & PT-0771 & $\begin{array}{l}\text { PT- } 0771 \text { provides measurement of the shellside pressure for } \\
\text { filter bundle } 4 \text {. }\end{array}$ & psig \\
\hline \multirow[t]{3}{*}{5} & PT-0779 & $\begin{array}{l}\text { PT- } 0779 \text { provides measurement of the tubeside inlet pressure } \\
\text { for filter bundle } 5 \text {. It is located at the entrance to filter bundle } 5 \text {. }\end{array}$ & psig \\
\hline & PT-0789 & $\begin{array}{l}\text { PT- } 0779 \text { provides measurement of the tubeside outlet pressure } \\
\text { for filter bundle } 5 \text {. It is located at the exit from filter bundle } 5 \text {. }\end{array}$ & psig \\
\hline & PT-0781 & $\begin{array}{l}\text { PT- } 0781 \text { provides measurement of the shellside pressure for } \\
\text { filter bundle } 5 \text {. }\end{array}$ & psig \\
\hline
\end{tabular}

Permeate mass flowrates are measured by Coriolis flow meters. A summary of these instruments is provided in Table 3.5 .

Table 3.5. PEP Coriolis Flow Meters for Permeate Production Rate Measurement

\begin{tabular}{cllc}
\hline Filter Bundle & Instrument ID & \multicolumn{1}{c}{ Description } & Units \\
\hline 1 & FT-0720 & $\begin{array}{l}\text { Coriolis flow meter FT-0720 measures permeate production rate } \\
\text { on filter bundle 1. }\end{array}$ & $\mathrm{kg} / \mathrm{min}$ \\
2 & FT-0755 & $\begin{array}{l}\text { Coriolis flow meter FT-0755 measures permeate production rate } \\
\text { on filter bundle 2. }\end{array}$ & $\mathrm{kg} / \mathrm{min}$ \\
3 & FT-0765 & $\begin{array}{l}\text { Coriolis flow meter FT-0765 measures permeate production rate } \\
\text { on filter bundle 3. }\end{array}$ & $\mathrm{kg} / \mathrm{min}$ \\
4 & FT-0775 & $\begin{array}{l}\text { Coriolis flow meter FT-0775 measures permeate production rate } \\
\text { on filter bundle 4. }\end{array}$ & $\mathrm{kg} / \mathrm{min}$ \\
5 & FT-0785 & $\begin{array}{l}\text { Coriolis flow meter FT-0785 measures permeate production rate } \\
\text { on filter bundle 5. }\end{array}$ & $\mathrm{kg} / \mathrm{min}$ \\
\hline
\end{tabular}


Correction of permeate production rates for variations in process temperature are made against the temperature of Tank T02A. This vessel is fitted with an array of RTDs to determine the tank temperature profile. All permeate flux corrections were made using the prototypic temperature sensor TTK-0619. Other temperature sensors of interest are installed in thermowells located along the filter loop. These are:

- TT-0791 - indicates the inlet temperature to filter bundle 1

- TT-0537 - indicates the outlet temperature from filter bundle 5

- TT-0513 - indicates the outlet temperature for HX-T02A

- TT-0515 - indicates the outlet temperature for HX-T03A

The energy required to pump the slurry also causes temperature rise in the filtration loop. Although the spiral plate heat exchanger (UFP-HX-T02A) removes this heat, temperature differentials (of a few degrees, depending on slurry consistency and yield stress) still exist between the filtration loop and Tank T02A. Because PEP is configured such that heat exchange occurs after the filter banks, it is likely that permeate going through the filters is several degrees warmer than the temperature measured in Tank T02A (which is the basis for temperature corrections). This is not an issue in the CUF filtration system because, in the bench-scale test apparatus, heat exchange occurs before filtration such that filtration and slurry reservoir temperature are similar. While it would be more appropriate to correct filter flux using the filtration loop temperature, temperature sensors in the filtration loop appear to be subject to potential stagnation of the slurry at the sensor thermowells (see the results for the High-Solids Scaling Test in Section 5.3.1). ${ }^{(a)}$ Because of these concerns, the tank temperature was selected as the temperature reference for filtration calculations.

The output signal from each of the PEP sensors listed in Table 3.3 to Table 3.5 was recorded by and stored in the PEP data acquisition system (DAS). The analog-to-digital conversion system has been calibrated to accurately convert the instrument signals and were stored in a read only data file to confirm the integrity of the process data from each test. The recorded data were time stamped by the DAS system so that it could be matched to process data sheets and logbooks. Conversion of the raw, stored instrument outputs to engineering data was carried out by a data interrogation program, which was technically reviewed, validated, and verified according to QA-RPP-WTP-SCP, Software Control. For simplicity, the system for recording, storing, and converting data will be hereafter referred to as the PEP DAS.

The control systems for PEP are extensive and include instrumentation, utility interfaces, chemical mixing and addition, process operations, valve alignment, and maintenance. The system is documented in the Tessenderlo Kerley Services Mechanical Data Book(s) Volumes I through XVIII as supplied by Bechtel National Inc. Testing processes were controlled by the test plan, the specific test instruction for the test being conducted, and the operating procedures for the PEP.

(a) NCR 42402.1. 
Table 3.6. PEP Test Operational Procedure Summary

\begin{tabular}{|c|c|}
\hline Administrative Procedures & Processing Procedures Continued \\
\hline$\overline{\text { OP-601 Surveillance Report Procedure }}$ & OP-413 Flushing and Transfer of Inhibited Water Procedure \\
\hline OP-602 Turnover Checklist Procedure & $\begin{array}{l}\text { OP-414 UFP-VSL T01A \& B Recirculation Procedure } \\
\text { OP-415 Stable Level Measurements Procedure }\end{array}$ \\
\hline \multicolumn{2}{|l|}{ System Startup and Shutdown Procedures } \\
\hline$\overline{\text { OP-101 System Startup Procedure }}$ & Process Maintenance Procedures \\
\hline OP-102 System Standby Shutdown Procedure & OP-501 Filter Chemical Cleaning Procedure cancelled - \\
\hline \multirow[t]{2}{*}{ OP-103 System Cold Shutdown Procedure } & requires revision \\
\hline & OP-502 Pump Seal Pot Operating Procedure \\
\hline Utilities Procedures & OP-503 Spill Response Procedure \\
\hline OP-201 Reverse Osmosis System Procedure & OP-504 Inspection of Vessel Interiors Using Video Camera or \\
\hline OP-202 Demineralized Water Procedure & Borescope \\
\hline \multicolumn{2}{|l|}{ SOP-203 Chilled Water System Operating Procedure (F\&O) } \\
\hline SOP-204 Compressed Air Operating Procedure (F\&O) & System Valve Alignment Procedures \\
\hline OP-205 Air Dryer Operating Procedure cancelled & Z001 HLP-VSL-T22 System Alignment Checklist \\
\hline SOP-206 Vacuum System Operating Procedure (F\&O) & Z002 FRP-VSL-T01 System Alignment Checklist \\
\hline OP-207 Vessel Vent System Procedure & Z003 UFP-VSL-T01A System Alignment Checklist \\
\hline SOP-208 Boiler Operating Procedure (F\&O) & Z004 UFP-VSL-T01B System Alignment Checklist \\
\hline \multirow[t]{2}{*}{ OP-209 Data Acquisition System (DAS) Operating Procedure } & Z005 UFP-HX System Alignment Checklist \\
\hline & Z006 UFP-VSL-T02A System Alignment Checklist \\
\hline Chemical Reagents Procedures & Z007 Ultrafilters System Alignment Checklist \\
\hline$\overline{\text { OP-301 Simulant Addition Procedure }}$ & Z008 UFP-VSL-T62A/B System Alignment Checklist \\
\hline OP-302 19M NaOH Operating Procedure & Z009 FEP-VSL-T01 System Alignment Checklist \\
\hline OP-303 Inhibited Water Procedure & Z010 HLP-VSL-T27 System Alignment Checklist \\
\hline OP-304 2M Caustic Operating Procedure & Z011 Vessel Vent System Alignment Checklist \\
\hline OP-305 Acid Procedure & Z012 Vacuum and Compressed Air System Alignment \\
\hline OP-306 Sodium Permanganate $(\mathrm{NaMnO} 4)$ Operating & Checklist F\&O \\
\hline Procedure & Z013 Boiler System Alignment Checklist F\&O \\
\hline \multirow[t]{2}{*}{ OP-307 Miscellaneous Chemical Addition Procedure } & Z014 Chemical System Alignment Checklist \\
\hline & Z015 Chemical System Alignment Checklist \\
\hline Processing Procedures & Z016 UFP-VSL-T01A PJM System Alignment Checklist \\
\hline 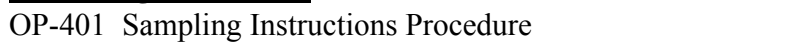 & Z017 UFP-VSL-T01B PJM System Alignment Checklist \\
\hline OP-402 Tank Transfer Procedure & Z018 UFP-VSL-T02A PJM System Alignment Checklist \\
\hline OP-403 Tank Heating and Cooling Procedure & Z019 Sparger System Alignment Checklist \\
\hline OP-404 Pulse Jet (PJM) Operating Procedure & Z020 UFP-VSL-T01A Coriolis Densitometer System \\
\hline OP-405 T02A Sparging Procedure & Alignment Checklist \\
\hline OP-406 Leaching Procedure & Z021 UFP-VSL-T01B Coriolis Densitometer System \\
\hline OP-407 T02A Leaching Procedure & Alignment Checklist \\
\hline OP-408 Tank T02A Recirculation Procedure & Z022 UFP-VSL-T02A Coriolis Densitometer System \\
\hline OP-409 Ultrafiltration (Dewatering) Procedure & Alignment Checklist \\
\hline OP-410 Solids Washing Procedure & Z023 Pump Seal Pot System Alignment Checklist \\
\hline OP-411 Tank Drain Operating Procedure & Z024 Pump Seal Pot System Alignment Checklist \\
\hline \multirow[t]{2}{*}{ OP-412 Waste Transfer Procedure } & Z025 Chilled Water System Alignment Checklist F\&O \\
\hline & Z026 Reverse Osmosis Unit System Alignment Checklist \\
\hline
\end{tabular}




\subsubsection{Cold-CUF Instrumentation}

Key components for measuring filtration performance on the cold-CUF test system match those for PEP. The cold-CUF test system includes instrumentation for measuring 1) filter AV (or slurry flowrate), 2) filter transmembrane and axial pressure drop, and 3) rate of permeate production and density. Table 3.7 provides a summary of select CUF process instrumentation relevant to the current study.

Table 3.7. CUF Filtration System Measurement Instrumentation

\begin{tabular}{ll}
\hline Parameter & Units \\
\hline Slurry Reservoir Temperature & ${ }^{\circ} \mathrm{C}$ \\
Permeate Pressure & psig \\
\hline Filter Inlet Pressure & psig \\
Filter Outlet Pressure & psig \\
\hline Filter TMP & psid \\
Volumetric Slurry Flow & $\mathrm{GPM}$ \\
\hline Filter AV & $\mathrm{ft} / \mathrm{s}$ \\
\hline Permeate Flow & $\mathrm{mL} / \mathrm{min}$ \\
\hline Permeate Density & $\mathrm{g} / \mathrm{mL}$ \\
\hline
\end{tabular}

Most of the sensors on the cold-CUF testing apparatus transmit analog data to an external dataacquisition collection system (DACS) from the National Instruments Corporation (Austin, TX). This system relays the analog data to a LabView data collection program. The software program scales the analog data, simultaneously records the data electronically, and displays it on the computer monitor. Figure 3.12 shows a diagram of the electronic sensors attached to the DACS.

\subsubsection{Analytical Analysis}

Filtration testing for both PEP and cold-CUF test systems involves sampling and analytical testing of waste simulant slurries (and their supernates). Analyses relevant to filtration performance include measurement of:

- slurry and supernate rheology (i.e., yield stress, consistency, and/or viscosity)

- slurry total solids (TS), undissolved solids (UDS), dissolved solids (DS), and centrifuged solids (CS) concentrations

- slurry and supernate densities.

Measurement of slurry and supernate rheologies was done by PNNL using an Anton Parr MCR 301 rheometer with a concentric cylinder geometry operated in a controlled-rate mode. Rheology was characterized through flow curve tests that measured the stress response of the fluid as a function of applied shear rate. Tests consisted of three segments. During the first segment, the shear rate was ramped from 0 to $1000 \mathrm{~s}^{-1}$ over $5 \mathrm{~min}$. During the second segment, the shear rate was held constant at $1000 \mathrm{~s}^{-1}$ for 1 minute. In the final segment, the shear rate was decreased from $1000 \mathrm{~s}^{-1}$ to 0 over 5 
minutes. Measurement data were recorded and subsequently analyzed with the RHEOPLUS/32 V3.21 software. This software allowed determination of the slurry or supernate sample's yield stress, consistency, and/or viscosity.
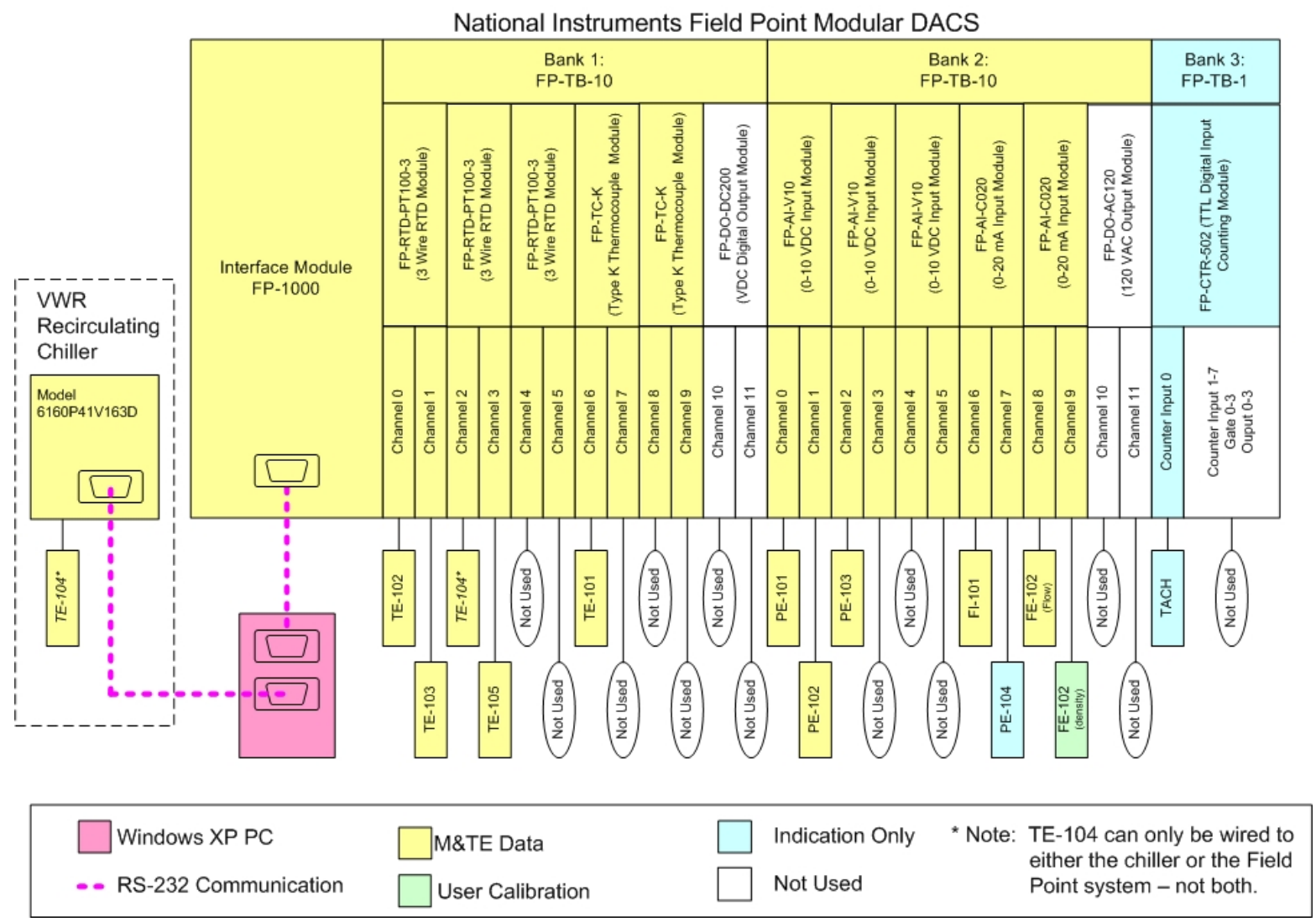

Figure 3.12. Diagram of DACS System

For measurement of slurry solids concentrations (including TS, UDS, DS, and CS) and slurry and supernate densities, samples were shipped to the Southwest Research Institute for analysis. Southwest Research Institute (SWRI) performed measurement of these physical properties using methods based on Guidelines for Performing Chemical, Physical, and Rheological Properties Measurements (24590-WTPGPG-RTD-001 Rev. 0). ${ }^{\text {(a) }}$

\subsubsection{Analysis of PEP DAS Data}

PEP filtration process conditions, such as TMP, axial pressure drop, and filter flux, can be defined for each filter bundle. In addition, the circulation loop filter AV can be defined from flow readings at the pump suction or discharge. A total system filter flux can be defined as well.

(a) GL Smith and K Prindiville. 2002. Guidelines for Performing Chemical, Physical, and Rheological Properties Measurements. 24590-WTP-GPG-RTD-001 Rev. 0, Bechtel National, Inc., Richland, Washington. 
The AV inside each filter element is calculated by dividing the volumetric slurry flow of the circulation loop by the total filter cross-section area:

$$
A V=\frac{Q_{s}}{S_{a}}=\frac{Q_{s}}{N\left(\frac{\pi}{4} D_{\text {inner }}^{2}\right)}
$$

where $A V=\mathrm{AV}$ in each filter

$S_{a}=$ cross sectional area of the axial flow

$Q_{s}=$ volumetric flowrate of slurry through the circulation loop

$N=$ number of filters per bundle (12 for all filters)

$D_{\text {inner }}=$ inner diameter of a single filter element ( 0.5 inches for all filter elements).

Since the cross-sections of the filters are all geometrically the same, a single AV can be defined for all filter bundles. AV can be determined using the circulation loop volumetric flowrate measurements taken by FT-0623 (suction to pump T42A) or FT-0635 (discharge from pump T43A). Although AVs based on both flow meters are sometimes reported, calculations of AV for PEP were typically based only on the high pressure flow meter reading (i.e., FT-0635).

The pressure differential between tubeside of the filter element and the shell filter bundle is commonly called the TMP. For PEP, TMP is calculated separately for each filter bundle using,

$$
\operatorname{TMP}(i)=\frac{\left[P_{\text {inlet }}(i)+P_{\text {outlet }}(i)\right]}{2}-P_{\text {shell }}(i)
$$

where $T M P(i)=$ TMP for filter bundle $i$

$P_{\text {inlet }}(i)=$ tube-side inlet pressure for filter bundle $i$

$P_{\text {outlet }}(i)=$ tube-side outlet pressure for filter bundle $i$

$P_{\text {shell }}(i)=$ shell pressure for filter bundle $i$

$i=$ filter bundle number (1-5).

Calculation of TMP for each filter bundle uses the pressure sensors listed in Table 3.4.

The pressure differential between the inlet and outlet of the filter element is called the axial pressure drop (APD). An APD can be determined for each filter bundle using,

$$
A P D(i)=P_{\text {inlet }}(i)-P_{\text {outlet }}(i)
$$

where $A P D(i)=$ axial pressure drop for filter bundle $i$

$P_{\text {inlet }}(i)=$ tube-side inlet pressure for filter bundle $i$

$P_{\text {outlet }}(i)=$ tube-side outlet pressure for filter bundle $i$

$i=$ filter bundle number (1-5).

Calculation of APD for each filter bundle uses the pressure sensors listed in Table 3.4. 
The PEP DAS records the mass flowrate of permeate produced by each filter bundle. The first step in calculating filter flux is to determine the volumetric flowrate of permeate produced. For filter $i$, the volumetric flowrate is calculated using the equation:

$$
Q_{p}(i)=\frac{G(i)}{\rho_{p}}
$$

where $Q_{p}(i)=$ volumetric flowrate of permeate from filter $i$

$G(i)=$ mass flowrate of permeate from filter $i$

$\rho_{p}=$ permeate density

$i=$ filter bundle number (1-5).

When the temperature of the slurry was not exactly $25^{\circ} \mathrm{C}$, the permeate flux rate was corrected to $25^{\circ} \mathrm{C}$ using (Geeting et al. 2003):

$$
Q_{t}(i)=Q_{p}(i) \exp \left[2500\left(\frac{1}{T+273}-\frac{1}{298}\right)\right]
$$

where $Q_{t}(i)$ is the corrected volumetric flowrate at $25^{\circ} \mathrm{C}$, T is the temperature $\left({ }^{\circ} \mathrm{C}\right)$, and $i$ is the filter bundle number (1-5).

As discussed in WTP-RPT-168, this equation corrects for both changes in permeate viscosity and cake structure with temperature (Daniel et al. 2009). The slurry temperature used was based on the prototypic temperature RTD in Tank T02A (i.e., TTK-0619) In addition, corrections for deviations in the TMP from the target value were also applied using:

$$
Q_{c}(i)=\left[\frac{T M P(i)}{T M P_{t}(i)}\right] \cdot Q_{t}(i)
$$

where $Q_{c}(i)$ is the TMP- and temperature-corrected volumetric flowrate, $\mathrm{TMP}_{\mathrm{t}}(i)$ is the target TMP for filter bundle $i$, and $i$ is the filter bundle number (1-5). TMP targets were 40 psid for all tests.

Equation 3.6 is intended to correct for "true" (persistent) deviations in TMP from its target value. It should not be used to correct for instrument and process noise. As such, it is applied only to process pressure measurements that have been time averaged over 1 minute intervals for the current report. The introduction for each of the scaling tests in Section 5.0 provides a description of how PEP data (including pressure measurements) are time averaged to damp out random process variation and measurement noise.

After temperature and TMP corrections, the filter flux for filter $i$ may be determined by:

$$
J(i)=\frac{Q_{c}(i)}{A(i)}
$$

where $J(i)$ is the filter flux of filter elements in filter bundle $i, A(i)$ is the total surface area of filter elements in filter bundle $i$, and $I$ is the filter bundle number (1-5). The filter surface area $A(i)$ is calculated from the known length of the filter bundle L(i) using the equation: 


$$
A(i)=\pi N D_{\text {inner }} L(i)
$$

Finally, a total (average) filter flux for all five filter bundles $\left(J_{t o t}\right)$ may be determined using:

$$
J_{\text {tot }}=\frac{\sum_{i} Q_{c}(i)}{\sum_{i} A(i)}
$$

\subsubsection{Analysis of CUF DACS Data}

The cold-CUF test system contains only a single 2-ft filter element. As such, process conditions, such as $A V, T M P, A P D$, and $J$, are defined only for the single element. The filter flux is defined as:

$$
J=\frac{Q_{c}}{A}
$$

where $J$ is the filter flux (and is most comparable to $J_{\text {tot }}$ from the PEP calculations), $Q_{c}$ is the temperatureand TMP-corrected volumetric permeate flowrate, and $A$ is the filtration surface area.

The filter area is calculated using:

$$
A=\pi D_{\text {inner }} L
$$

where $D_{\text {inner }}$ is the filter element inner diameter ( 0.5 inches $)$, and $L$ filter element length $(2 \mathrm{ft})$.

As with the PEP system, the permeate volumetric flowrate and/or filter flux is corrected for deviations in 1) slurry temperature from the target test temperature (typically $25^{\circ} \mathrm{C}$ ) and 2 ) TMP from the target TMP (typically 40 psid) using:

$$
\begin{gathered}
Q_{t}=Q_{p} \exp \left[2500\left(\frac{1}{T+273}-\frac{1}{298}\right)\right] \\
Q_{c}=\left[\frac{T M P}{T M P_{t}}\right] \cdot Q_{t}
\end{gathered}
$$

Here $Q_{p}$ is the uncorrected permeate volumetric flowrate (provided directly by the CUF DAQS). The TMP is calculated using:

$$
T M P=\frac{\left(P_{\text {inlet }}+P_{\text {outlet }}\right)}{2}-P_{\text {permeate }}
$$

where $P_{\text {inlet }}$ is the pressure at the filter inlet, $P_{\text {outlet }}$ is the pressure at the filter outlet, and $P_{\text {permeate }}$ is the pressure at the permeate side of the filter. 
As before, Equation 3.13 should only be applied in cases where TMP deviation is persistent and not simply a result of process or measurement noise. The introduction for each of the scaling tests in Section 5.0 provides a description of how PEP data (including pressure measurements) are time averaged to dampen out random process variation and measurement noise.

The AV inside the filter is calculated by dividing the volumetric slurry flow of the filter by the cross section area of the inside diameter of the filter:

$$
A V=\frac{Q_{s}}{\frac{\pi}{4} D_{\text {inner }}^{2}}
$$

where $Q_{s}$ is the volumetric slurry flowrate in the axial direction.

\subsubsection{Analysis of Dewatering Curves}

Overall filter behavior is modeled by the Darcy equation, which describes filter flux as:

$$
J=\frac{\Delta P_{m}}{\mu_{\text {permeate }} R}
$$

where $\Delta P_{m}$ is the pressure drop across filter membrane (or TMP), $\mu_{\text {permeate }}$ is the viscosity of the permeate, and $R$ is the overall resistance of the filter membrane to permeation'

The overall filter resistance term is a sum of the resistance of the actual filter, the resistance of the filter cake that forms on the surface of the filter, and the resistance due to fouling of the filter.

The dependence of the overall filter resistance on slurry solids concentration is key for assessing the dewatering behavior. A typical dependence observed during dewatering operation of Hanford tank waste simulants is shown in Figure 3.13. For dilute slurries and when turbulent flow conditions exist, the filter resistance is usually constant and characterized by the resistance of the porous filter element $\left(R_{m}\right)$ such that:

$$
J \sim \frac{\Delta P_{m}}{\mu_{\text {permeate }} R_{m}}
$$

For filtration in this regime, the TMP and permeate viscosity are the controlling operational parameters.

At the higher slurry solids concentrations that occur during washing and dewatering operations, the filter cake resistance plays a more significant role in determining filter flux. The filter cake resistance is dependent on system operational properties like AV. Treatment of filtration data against the Darcy equation is complicated by the need to account for the dependence of filter cake resistance on AV and slurry concentration. Ultimately, the slurry can only be dewatered to a maximum UDS concentration limit at a given TMP. This limiting concentration is known as the gel concentration and is typically similar to a slurry's centrifuged solids concentration. As the simulant slurry's solid concentration approaches the gel concentration, the filter flux can be described as: 


$$
J=k \cdot \ln \left[\frac{C_{s}}{C_{g}}\right]
$$

where $C_{s}$ is the slurry UDS concentration, $C_{g}$ is the slurry gel concentration at a given TMP, and $k$ is the constant for a given TMP and AV (note that $\mathrm{k}$ is a negative value and is typically termed the "mass transfer coefficient).

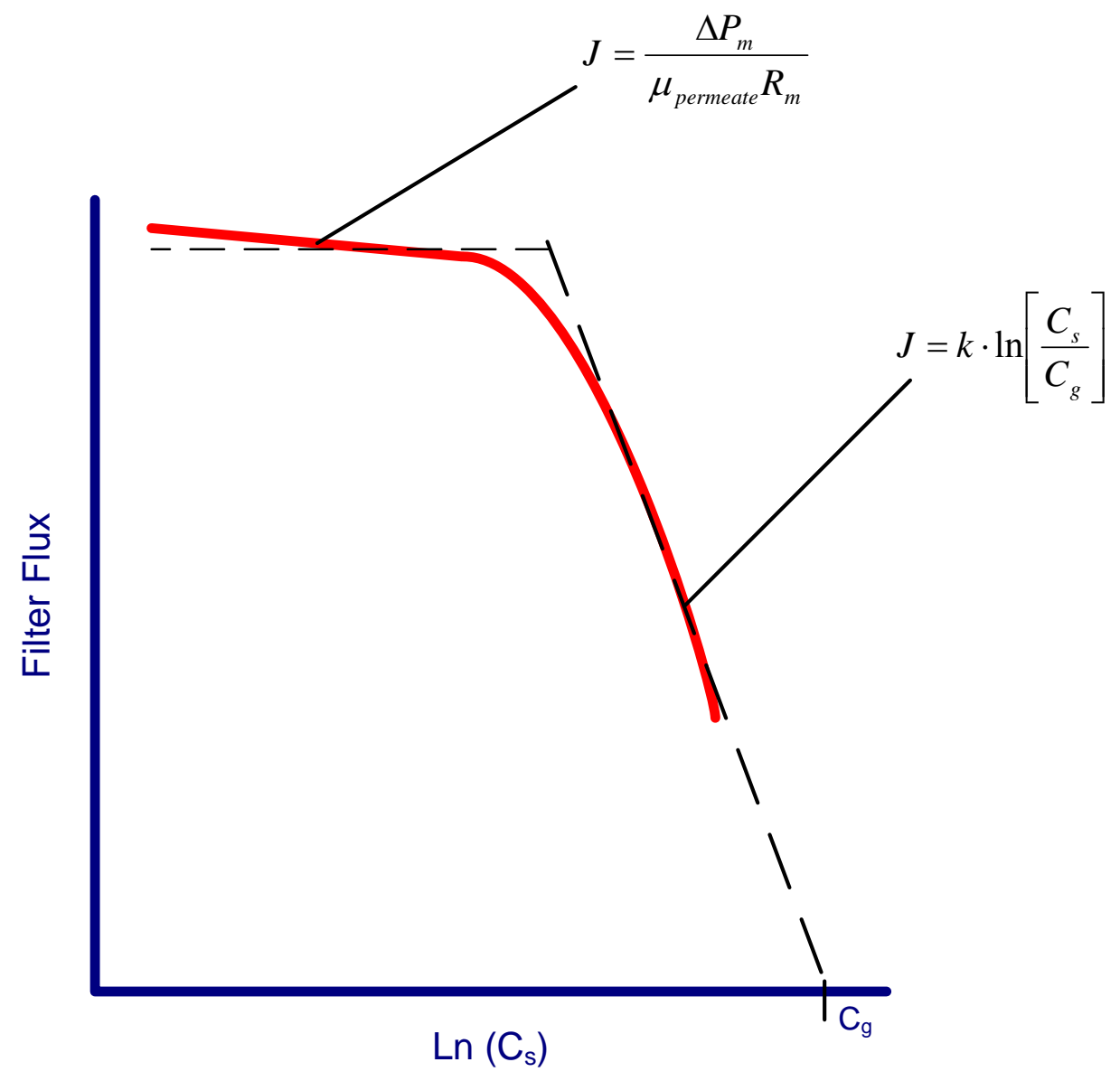

Figure 3.13. Typical Filter Flux Behavior as a Function of Solids Concentration

Dewatering operations affect a change in the slurry UDS concentration by removing permeate from the slurry. Unlike continuous recycle filtration, the collected permeate is not returned to the slurry reservoir. Because both PEP and cold-CUF measure the rate of permeate production, it is possible, given the known starting mass and concentration of circulating slurry, to estimate the UDS as a function of time. The circulating slurry is defined as that contained in the slurry reservoir (i.e., mixing tank) and filtration loop. If filtration is assessed at several equally spaced time intervals $(\Delta t)$, then for a given time step $n$, the slurry UDS concentration $\mathrm{x}(\mathrm{n})$ can be determined using:

$$
x(n)=\frac{m_{U D S}}{m_{T}(n)}
$$


where $m_{U D S}$ is the mass of undissolved solids, $m_{T}(n)$ is the total mass of circulating of slurry remaining in the filter loop, and $n$ is the time interval.

Filtration is assumed 1) to retain all slurry solids and 2) proceed with no dissolution of slurry solids such that:

$$
m_{U D S}=x_{o} m_{T, o}
$$

where $\mathrm{x}_{\mathrm{o}}$ is the initial UDS concentration, and $\mathrm{m}_{\mathrm{T}, \mathrm{o}}$ is the mass of the circulating slurry (given process parameters).

The mass of circulating slurry at time interval $n$ is given by:

$$
m_{T}(n)=m_{T}(n-1)-G(n) \cdot \Delta t
$$

Here, $G(n)$ is the mass flowrate of permeate and is measured by the PEP Coriolis permeate flow meters or, for CUF, is determined by the permeate volumetric flowrate $(Q)$ and measured permeate density $\left(\rho_{\mathrm{p}}\right)$ using:

$$
G(n)=\rho_{p}(n) \cdot Q(n)
$$

The circulating slurry mass may also be expressed in terms of the original slurry mass using:

$$
m_{T}(n)=m_{T, o}-\Delta t \cdot \sum_{j=1}^{n} G(j)
$$

Thus, the slurry UDS concentration at time interval $\mathrm{n}$ may be expressed in terms of the previous permeate mass flowrates:

$$
x(n)=\frac{x_{o} m_{T, o}}{m_{T, o}-\Delta t \cdot \sum_{j=1}^{n} G(j)}
$$

\subsubsection{Slurry Solids-to-Filter Surface Area Ratio}

Comparative studies of filtration at two-different scales are often facilitated by scaling filtration such that there is a similar volume (or mass) of slurry for the given filter surface area employed. The ratio of slurry solids-to-filter area $(S A)$ is defined as:

$$
S A=\frac{x_{o} m_{T, o}}{A}
$$

Here, $x_{o}$ and $m_{T, o}$ are the initial slurry UDS concentration and total slurry mass. Thus, for many filtration studies (including those reported herein), the slurry mass for different scale tests is selected to 
provide a comparable (similar) $S A$ value at different scales. For the current document, $S A$ is reported in units of $\mathrm{kg}$ (or solids) per square foot $\left(\mathrm{ft}^{2}\right)$ of filter area used for testing.

The importance of maintaining SA between scale tests depends on simulant properties (such as PSD and settled solids strength) that affect cake formation and the propensity of fines to foul the filter. For the simulant slurry used in PEP testing, Daniel et al. (2009) provides insight into how differences in $S A$ between test scales may impact testing. In particular, Daniel et al. (2009) examined filtration on 2-ft-long and 8-ft-long filters on a bench-scale CUF filtration system. Both 2-ft and 8-ft tests employed the similar test volumes, and as such, the SA of the 8 - $\mathrm{ft}$ test was approximately 4 times lower than that of the 2 -ft test. Despite the difference in $S A$ in $2-\mathrm{ft}$ and 8 - $\mathrm{ft}$ tests, the rate of filtration appeared to scale directly with filter length from 2 -ft to 8 -ft scales (i.e., the filter flux was the same in both tests). The only noticeable difference in the flux behavior that occurred as a result of the difference in $S A$ was that depth fouling of the 8-ft filter occurred more slowly than that in the 2-ft filter. As such, this suggests that filtration experiments that employ simulants developed for PEP should not be impacted by minor $(<10 \%)$ or moderate $(<50 \%)$ differences in the $S A$ used for scale testing.

\subsubsection{Scaling Factor Analysis}

Scaling factor analysis is intended to provide a measure of how process performance measured using the bench-scale cold-CUF filtration system compares to that measured on the engineering-scale PEP filtration system. As the current study considers both low-solids continuous recycle filtration operations and high-solids dewatering operations approaching the limiting gel concentration (see Section 4.0 for the experimental approach to scaling evaluations), scaling factor analyses are defined for both test approaches.

To compare process scaling of low-solids continuous recycle filtration tests, CUF flux is compared directly to PEP flux. Scaling factors can be evaluated by comparing individual PEP filter flux $[J(i)]$ to the CUF flux or by comparing the total PEP flux $\left[J_{\text {tot }}\right]$ to that measured on the cold-CUF. The scaling factor is defined as the ratio of PEP flux to CUF filter flux. Thus, when considered on an individual PEP filter bundle basis, the PEP to CUF scaling factor for filter bundle $i$ is given by:

$$
S(i)=\frac{J(i)}{J_{C U F}}
$$

Here, $J_{C U F}$ is the flux as measured by the cold-CUF test system, and $\mathrm{S}(i)$ is used to denote the individual flux scaling factor for filter bundle $i$.

When considered on a total flux basis, the scaling factor is given by:

$$
S_{\text {tot }}=\frac{J_{\text {tot }}}{J_{C U F}}
$$

where $S_{\text {tot }}$ denotes the total PEP to CUF scaling factor.

For both definitions, the low-solids scaling factors can be interpreted as follows:

- $S=1$, indicates similar filtration performance (rate) in CUF and PEP 
- $S<1$, indicates that CUF over predicts PEP filtration performance (rate)

- $S>1$, indicates that CUF under predicts PEP filtration performance (rate).

At a given AV and TMP, dewatering curves for solids slurries approaching their limiting gel concentration can be characterized in terms of their mass transfer coefficient, $k$, and limiting gel concentration, $C_{g}$. Thus for the high-solids dewatering tests, the scaling factor is also defined in these terms. Specifically, high-solids dewatering is characterized by two separate scaling factors-one based on $k$ and the other based on $C_{g}$. The $k$ scaling factor (denoted by $S_{k}$ ) is defined as:

$$
S_{k}=\frac{k_{P E P}}{k_{C U F}}
$$

where $k_{P E P}$ and $k_{C U F}$ are the mass transfer coefficients for PEP and CUF dewatering curves, respectively.

Thus, the scaling factor for the mass transfer coefficient $\left(S_{k}\right)$ can be interpreted as follows for a gel limited dewatering regime:

- $S_{k}=1$, indicates similar decline in CUF and PEP filter flux with increasing solids concentration

- $S_{k}<1$, indicates that CUF over predicts the decline in filter flux (relative to PEP) with increasing solids concentration

- $S_{k}>1$, indicates that CUF under predicts the decline in filter flux (relative to PEP) with increasing solids concentration.

Likewise, the $C_{g}$ scaling factor (denoted by $S_{g}$ ) is defined as:

$$
S_{g}=\frac{C_{g, P E P}}{C_{g, C U F}}
$$

where $C_{g, P E P}$ and $C_{g}$, CUF are the limiting gel concentrations for PEP and CUF dewatering curves, respectively.

Thus, the scaling factor for the gel concentration $\left(S_{g}\right)$ can be interpreted as follows for a gel limited dewatering regime:

- $S_{g}=1$, indicates a similar limiting gel concentration for PEP and CUF dewatering operations

- $S_{g}<1$, indicates that CUF over predicts the limiting gel concentration for dewatering operations (relative to PEP)

- $S_{g}>1$, indicates that CUF under predicts the limiting gel concentration for dewatering operations (relative to PEP).

As discussed in Section 3.4.7, Daniel et al. (2009) examined bench scale-filtration using 2-ft and 8-ft long filter elements with waste simulant slurries similar to that used in PEP. It provides insight on what to expect from the PEP to CUF scale testing reported herein. In particular, Daniel et al. (2009) found that 
scaling effects were minimal during low-solids concentration continuous recycle experiments and slurry dewatering operations, even when the slurries were tested at dissimilar $S A$ relative to their scale. As such, when only total filtration length issues are considered, the PEP to CUF scaling factor for continuous recycle and dewatering operations should be close to 1.0. However, the scaling results in Daniel et al. (2009) can only be directly applied to the CUF comparisons. As such, it may not capture scaling effects that derive from PEP design and layout. Examples of design and layout unique to PEP that could impact scaling include filter entrance length, geometry effects, and filter bundling effects. 


\subsection{Scaling Test Experimental}

The objective of the current report is to evaluate the scale-up performance of the filtration process for WTP. To this end, the filtration performance of a Hanford tank waste simulant was evaluated at both engineering- and bench-scales. Engineering scale filter performance tests were conducted at the PEP under test plan TP-RPP-WTP-506. ${ }^{\text {(a) }}$ Parallel bench-scale tests were conducted under test plan TP-WTPPEP-044 ${ }^{(\mathrm{b})}$ using the cold-CUF filtration system located at APEL. Test Plan TP-RPP-WTP-506 ${ }^{(\mathrm{a})}$ outlines numerous tests that explore performance of waste simulants in the full range of WTP pre-treatment operations, including vessel-to-vessel waste transfers, caustic-leaching operations, oxidative-leaching operations, and slurry dewatering and washing operations. The tests aimed solely at determining filter scale-up performance represent a small subset of the overall test array outlined in TP-RPP-WTP-506. ${ }^{(a)}$

In the following sections, the filter scaling tests and waste simulant slurry properties are described in detail. Three separate scaling tests were performed to assess scaling effects that exist between PEP engineering-scale filtration operations and cold-CUF, bench-scale filtration operations. These tests are:

- Low-Solids Scaling Test \#1: A 36-hr low-solids concentration continuous/backpulsed recycle filtration operation

- Low-Solids Scaling Test \#2: A repeat of the 36-hr low-solids concentration continuous/backpulsed recycle filtration operation

- High-Solids Scaling Test: A high-solids dewatering operation.

For each test run at the PEP, a parallel test was run on the cold-CUF filtration system located at APEL. These tests allow assessment of the PEP to CUF scaling factor for continuous and backpulsed recycle operations and for dewatering operations approaching the slurry gel point.

In the following sections, a description of the specific test steps and experimental setups used to perform each of the three scaling tests in PEP and in CUF are given. All cold-CUF scaling tests were typically stand alone processes with no other experimental objectives other than to reproduce the parallel PEP scaling experimental process. However, scaling tests for the PEP were a subset of tests assessing multiple aspects of WTP performance. As such, PEP scaling tests were often preceded by test steps associated with other experimental objectives. For this reason, the scaling test descriptions for PEP also include a brief summary of all preceding test steps.

\subsection{Low-Solids Scaling Test \#1}

The first low-solids scaling test was aimed at assessing the PEP to CUF scaling factor for a low-solids concentration (6.9 wt\% UDS), pre-leach, waste simulant slurry undergoing continuous and backpulsed recycle filtration. The test was composed of three 12-hr segments. During the first 12-hr segment, the slurry was filtered continuously in recycle mode (such that all permeate collected was returned to Tank T02A) without any filter backpulsing. During the second 12-hr segment, continuous recycle operations

(a) GB Josephson, OP Bredt, JK Young, and DE Kurath. 2009. Pretreatment Engineering Platform (PEP) Testing (Phase I). TP-RPP-WTP-506, Rev. 0.4, Pacific Northwest National Laboratory, Richland, Washington.

(b) RL Russell. 2008. Test Plan for the PEP Parallel Laboratory Testing. TP-WTP-PEP-044, Rev. 0.2, Pacific Northwest National Laboratory, Richland, Washington. 
were performed with backpulsing of the filter banks at 30 minute intervals. During the final 12-hr segment, the slurry was again filtered continuously in recycle mode with no filter backpulsing. The scaling factor was then determined by comparing the cold-CUF filter flux to the PEP filter flux over all three segments.

\subsubsection{PEP Operations for Low-Solids Scaling Test \#1}

PEP operations for Low-Solids Scaling Test \#1 were conducted as part of PEP Functional Testing activities in November 2008. The scaling test steps were in accordance with PEP Functional Test Steps A.1.8 to A.1.10 outlined in TP-RPP-WTP-506, Appendix A. (a) A summary of these steps (and simulant shakedown activities preceding these steps) is provided below.

\section{$\underline{\text { Test Steps Preceding Low-Solids Scaling Test \#1 }}$}

The filter elements were cleaned with oxalic acid before functional testing in November 2008. It should be noted that filter cleaning operations employing oxalic acid were not prototypic - plant operations are expected to use nitric acid to clean the filters. Oxalic acid for the Low-Solids Scaling Test \#1 was selected on the basis that bench-scale tests indicated that it more effectively cleaned the filters. Also, the intent of the cleaning operations preceding the low-solids scaling tests was to restore the filters to a clean condition to the best extent possible.

Cleaning operations took place over the $21^{\text {st }}$ and $22^{\text {nd }}$ of November. First, Tank T02A and the filtration loop (i.e., the heat exchangers, pulse pots, the shell- and tube-side of the filters, the piping, and all dead legs) were drained. Next, approximately 200 gal of $0.5 \mathrm{M}$ oxalic acid solution was loaded into the filter loop. This solution was circulated and filtered through all five filter bundles with a recycle of permeate back to T02A for 10 minutes, and it was subsequently backpulsed 30 times. Filtration was then run with the oxalic acid solution for 1 hour to obtain a stable filter flux. At the end of this filtration period, the oxalic acid solution was drained from the filters, and Tank T02A was filled with 180 gallons of water from a reverse osmosis purification unit (i.e., RO water). The RO water was circulated through the filters for 15 minutes, and the filters were then backpulsed 15 times. Next, the RO water was allowed to continue filtering for another 15 minutes, during which the filtration flux was monitored. Finally, the Tank T02A and the filtration loop were drained and refilled with another 180 gallons of RO water (pH adjusted to 3). This was circulated through the filters for 30 minutes to obtain a stable "clean" filter flux. A final backpulse of the system was performed before isolating the cleaned filters and draining Tank T02A and the filter piping and heat exchangers.

It should also be noted that before functional testing associated with Low-Solids Scaling Test \#1, the testing with PEP filter elements had been limited to water functional testing. As such, the PEP filters were relatively unused and had only limited filtration history.

To prepare for functional testing, a quantity of Phase 1 simulant sufficient to complete the simulant functional tests was prepared in HLP-VSL-T22. This batch was sampled to characterize simulant for the purpose of specifying all simulant-dependent process parameters (e.g., batch volumes, caustic and permanganate additions) for integrated tests conducted with this simulant batch. Next, a prototypic batch volume of the waste simulant slurry was transferred to UFP-VSL-T01A. Anti-foam agent was added to

(a) GB Josephson, OP Bredt, JK Young, and DE Kurath. 2009. Test Plan for Pretreatment Engineering Platform (PEP) Testing (Phase I). TP-RPP-WTP-506, Rev. 0.4, Pacific Northwest National Laboratory, Richland, Washington. 
the slurry in Tank T01A to control foaming. Waste simulant was transferred from vessel UFP-VSLT01A to vessel UFP-VSL-T02A. The volume of simulant transferred was sufficient to fill Tank T02A to the maximum batch level. The filter loop, pulse pots, and permeate lines had been filled with inhibited water as part of normal PEP filter lay-up operations. This inhibited water was drained (to the best extent possible) from the filter components. After draining was complete, the shell-side of the filters, the pulse pots, and the permeate lines were filled with simulant permeate (via filtration). It should be noted that the slurry becomes slightly dewatered when the permeate collection and metering systems are filled. The low solids scaling test was then executed.

\section{Low-Solids Scaling Test \#1 Operational Test Steps}

The first low-solids scaling test took place from November $23^{\text {rd }}$ to November $25^{\text {th }}, 2008$. As stated previously, the low solids scaling test consisted of three separate test operations:

- An initial $12 \mathrm{hr}$ continuous recycle filtration operation where the filters are operated without backpulsing (Functional Test Step A.1.8)

- A second $12 \mathrm{hr}$ continuous recycle filtration operation during which the filters are backpulsed a total of 24 times, with the backpulses occurring approximately every 30 minutes (Functional Test Step A.1.9)

- A final $12 \mathrm{hr}$ continuous filtration operation where the filters are operated without backpulsing (Functional Test Step A.1.10)

The target operational conditions for all three segments of the Low-Solids Scaling Test \#1 are listed in Table 4.1. The actual operational conditions achieved during testing are listed in Section 5.1.1. For the test, all five filter bundles were aligned. A slurry flowrate of $109 \pm 10$ GPM was targeted and corresponds to a filter AV of $15.0 \pm 1.4 \mathrm{ft} / \mathrm{s}$. A TMP of $40 \pm 4$ psid for all filters was also targeted. Slurry flow was aligned through UFP-HX-T02A so that any heat generated by the pumping of the slurry could be removed. A circulation loop temperature of $25 \pm 2^{\circ} \mathrm{C}$ was targeted. The steam heater (UFPHX-T03A) was bypassed. All permeate collected was recycled back to Tank T02A. For mixing of Tank T02A contents, UFP-VSL-T02A PJM velocities and cycle times were selected to match the pretreatment facility PJM specifications.

Table 4.1. Target Operational Conditions for Low-Solids Scaling Test \#1

\begin{tabular}{lc}
\hline \multicolumn{1}{c}{ Parameter } & Target \\
\hline Tank T02A PJM Jet Velocity & $7.3 \pm 0.4 \mathrm{~m} / \mathrm{s}$ \\
Tank T02A PJM Cycle Time & $33 \pm 1 \mathrm{~s}$ \\
Tank T02A PJM Stroke Length & $80 \pm 5 \%$ \\
& $30.3 \pm 1.8 \mathrm{inches}$ \\
Tank T02A Steam Ring Purge Flowrate & $0.10 \pm 0.02 \mathrm{~kg} / \mathrm{min}$ \\
Tank T02A Upper Air Sparger Flowrate & $0.10 \pm 0.02 \mathrm{~kg} / \mathrm{min}$ \\
Tank T02A Total Lower Air Sparger Flowrate & $0.40 \pm 0.05 \mathrm{~kg} / \mathrm{min}$ \\
Number of Filter-Loop Bundles & 5 \\
Filter AV & $15.0 \pm 1.4 \mathrm{ft} / \mathrm{s}$ \\
TMP & $40 \pm 4 \mathrm{psid}$ \\
Slurry Temperature & $25 \pm 2^{\circ} \mathrm{C}$ \\
Maximum Filter Bundle Trans-axial Pressure Drop & $25 \mathrm{psid}$ \\
\hline
\end{tabular}


Tank T02A and the filter circulation loop were sampled periodically throughout Low-Solids Scaling Test \#1 to determine the physical properties of the slurry. A summary of sampling during Low-Solids Scaling Test \#1 is provided in Table 4.2. The information provided in this table is based on actual sampling during the execution of the tests. Discrepancies exist between actual sampling and that prescribed in Test Plan TP-WTP-RPP-506. ${ }^{\text {(a) }}$

Table 4.2. A List of Slurry Sampling for Low-Solids Scaling Test \#1

\begin{tabular}{|c|c|c|}
\hline Functional Test Step & Occurrence & Sampling Level \\
\hline \multirow[t]{2}{*}{$\begin{array}{l}\text { A.1.8 - initial } 12 \mathrm{hr} \\
\text { filtration }\end{array}$} & $\begin{array}{l}\text { At start of the initial 12-hr } \\
\text { continuous operation (about } \\
30 \text { min after permeate was known } \\
\text { to be flowing back into Tank } \\
\text { T02A) }\end{array}$ & $\begin{array}{l}\text { Collected nine in-line samples for physical } \\
\text { properties testing from filtration loop in-line } \\
\text { sampler. Properties to be measured include } \\
\text { slurry UDS concentration, slurry density, } \\
\text { rheology, and particle size. This report does } \\
\text { not present particle-size results. }\end{array}$ \\
\hline & $\begin{array}{l}\text { During } 12 \text { hr initial continuous } \\
\text { operation } \\
\text { Note: The results of the samples } \\
\text { taken during this part of the test } \\
\text { are intended to assess sampler } \\
\text { variability. While this is not part } \\
\text { of the scope of this report, these } \\
\text { samples also provide physical } \\
\text { property information that can be } \\
\text { used to support low-solids } \\
\text { filtration test calculations. }\end{array}$ & $\begin{array}{l}\text { Collected a total of } 30 \text { filter-loop in-line } \\
\text { samples at } \sim 1 \text { hr intervals over a period of } 5 \\
\text { hrs for in-line sampling standard deviation } \\
\text { estimate. Samples were collected in batches of } \\
6 \text { and analyzed for slurry UDS concentration. } \\
\text { Collected a total of } 30 \text { samples from the } \\
\text { middle/middle CD port at } \sim 1 \mathrm{hr} \text { intervals over } \\
\text { a period of } 5 \mathrm{hrs.} \mathrm{Samples} \mathrm{were} \mathrm{collected} \mathrm{in} \\
\text { batches of } 6 \text {. Samples were collected in } \\
\text { parallel with the filter-loop in-line samples } \\
\text { and analyzed for slurry UDS concentration. In } \\
\text { some instances, there was difficulty in } \\
\text { obtaining samples from the middle/middle CD } \\
\text { port. As a result, the middle/low CD port was } \\
\text { used to obtain some of these } 30 \text { samples. }\end{array}$ \\
\hline $\begin{array}{l}\text { A. } 1.9-\text { backpulsed } 12 \\
\text { hr filtration }\end{array}$ & $\begin{array}{l}\text { Toward the end of } 12 \mathrm{hr} \\
\text { backpulsed operations (at the } \\
\text { completion of the last backpulse) }\end{array}$ & $\begin{array}{l}\text { Collected two samples from the filter-loop in- } \\
\text { line sampler port for characterization. These } \\
\text { samples were analyzed for slurry UDS } \\
\text { concentration and PSD. }\end{array}$ \\
\hline $\begin{array}{l}\text { A. } 1.10-\text { final } 12 \mathrm{hr} \\
\text { filtration }\end{array}$ & $\begin{array}{l}\text { Toward the end of the final } 12 \mathrm{hr} \\
\text { continuous operation }\end{array}$ & $\begin{array}{l}\text { Collected four samples from the filtration loop } \\
\text { in-line sampler port for characterization. } \\
\text { These samples should be analyzed for slurry } \\
\text { UDS concentration and PSD. This report does } \\
\text { not present particle-size results. }\end{array}$ \\
\hline
\end{tabular}

(a) GB Josephson, OP Bredt, JK Young, and DE Kurath. 2009. Test Plan for Pretreatment Engineering Platform (PEP) Testing (Phase I). TP-RPP-WTP-506, Rev. 0.4, Pacific Northwest National Laboratory, Richland, Washington. 


\subsubsection{CUF Operations for Low-Solids Scaling Test \#1}

Parallel bench-scale filtration tests used the cold-CUF filtration system at APEL. Tests for LowSolids Scaling Test \#1 were conducted in early December 2008. A low-solids simulant slurry (see Table 4.2, step A.1.8) delivered from the PEP was tested in a 36-hour filter conditioning test that included three equal 12 hour segments of 1) continuous non-backpulsed operation, 2) periodic backpulsing, and 3 ) continuous non-backpulsed operation. With the exception of a slight difference in UDS concentration ( $\sim 0.1$ to $0.2-w t \%$ resulting from filling of the CUF permeate metering system with supernate), the test slurry used for CUF operations had the same slurry composition (including anti-foam agent) as that used for PEP filtration testing. The testing proceeded according to these general steps:

1) Load the PEP simulant slurry into the CUF slurry reservoir.

2) Operate the CUF filtration system in recycle mode at target conditions of TMP $=40 \mathrm{psid}$ and $\mathrm{AV}=15 \mathrm{ft} / \mathrm{s}$ for 12 hours.

3) Operate the CUF filtration system in recycle mode at target conditions of TMP $=40 \mathrm{psid}$ and $\mathrm{AV}=15 \mathrm{ft} / \mathrm{s}$ and perform a single backpulse every 30 minutes for 12 hours.

4) Operate the CUF filtration system in recycle mode at target conditions of TMP $=40 \mathrm{psid}$ and $\mathrm{AV}=15 \mathrm{ft} / \mathrm{s}$ for 12 hours.

All tests were performed at a target reservoir temperature of $25 \pm 5^{\circ} \mathrm{C}$.

Prior to executing the test steps outlined in the preceding paragraph, the cold-CUF system was cleaned with a concentrated nitric acid solution and an oxalic acid solution. First, any slurry and permeate hold-up were drained from the system. The inside of the slurry reservoir was rinsed with deionized (DI) water to remove excess solids on the side of the tank walls and drain. The recirculation loop was then rinsed with DI water by pumping successive volumes of water through the loop and draining until the water exiting the system appeared to be clear. Next, nitric acid cleaning was performed by adding a $2 \mathrm{M}$ nitric acid $\left(\mathrm{HNO}_{3}\right)$ solution to the system. The solution was allowed to circulate in the CUF through both the slurry and permeates lines for approximately one hour. At the start and end of the cleaning, three backpulses were performed on the system. The acid solution was then drained from the slurry loop and permeate loop of the CUF. The loops were rinsed with DI water twice and once with 0.01 $\mathrm{M} \mathrm{NaOH}$ to remove excess acid out of the system. After draining the last rinse solution, a $0.5 \mathrm{M}$ solution of oxalic acid was added to further clean the filter. Like the nitric acid cleaning step, the solution was circulated through both the slurry loop and permeate loop for approximately one hour. Three backpulses were performed at the start and end of the cleaning. The solution was drained, and the loops were rinsed with DI water twice and $0.1 \mathrm{M} \mathrm{NaOH}$ once to remove the excess acid.

With regard to CUF filter history, the filter element employed for PEP parallel tests described in this report had also been used extensively for bench-scale simulant development and testing activities throughout calendar year 2008. A full list of previous CUF testing is beyond the scope of this report. A partial description of previous testing can be found in the simulant development reports for boehmite (Russell et al. 2009a), gibbsite (Russell et al. 2009b), and the filtration simulant (Russell et al. 2009c) as well as in the simulant testing report (Daniel et al. 2009). 


\subsection{Low-Solids Scaling Test \#2}

Low-Solids Scaling Test \#2 was performed during functional testing conducted from late December 2008 to early January 2009. The second scaling test was a repeat of Low-Solids Scaling Test \#1. As before, the objective of the second test was to assess the PEP to CUF scaling factor for a low-solids concentration waste simulant slurry (6.9 wt\% UDS) undergoing continuous and backpulsed recycle filtration. The same test scheme as outlined for the first low-solids scaling test was followed for LowSolids Scaling Test \#2. Because of this, discussion of the operations involved in the second low-solids scaling test is limited.

\subsubsection{PEP Operations for Low-Solids Scaling Test \#2}

The specific PEP operations associated with Low-Solids Scaling Test \#2 (including those that preceded the actual scaling tests) match those for Low-Solids Scaling Test \#1 discussed in Section 4.1.1 with a few exceptions. These are:

- Heat exchanger UFP-HX-T03A was bypass passed in the second test (but was aligned in the first test).

- Only Pump T42A was employed in the second test (both T42A and T43A were used in the first).

- The 30-in. line and CD samples were not taken in the second test.

The operational parameters and sampling schemes used for Low-Solids Scaling Test \#1 also apply to Low-Solids Scaling Test \#2. Table 4.3 and Table 4.4 provide summaries of the PEP operations and sampling schedules for the repeat low-solids scaling test. The actual operational conditions achieved during testing are listed in Section 5.2.1. In addition, the information provided in Table 4.4 is based on actual sampling during the execution of the tests. Discrepancies exist between actual sampling and that prescribed in Test Plan TP-WTP-RPP-506. ${ }^{(a)}$ For example, the 30 -in. line and Tank T02A samples pulled in accordance with the test plan (see Table 4.2) were not collected during Low-Solids Scaling Test \#2 because sampling for Low-Solids Scaling Test \#1 had addressed issues for which these samples were taken.

Before executing test steps associated with Low-Solids Scaling Test \#2, the filters were cleaned twice with oxalic acid. As before, the use of oxalic acid is not prototypic. Selection was based on the desire to clean the filters to the best extent possible (see Section 4.1.1 for additional discussion). The first cleaning event took place over December $27^{\text {th }}$ to $28^{\text {th }}, 2008$, and employed a $0.5 \mathrm{M}$ oxalic acid solution. This first oxalic acid cleaning event did not restore the "clean" water filter flux to desired levels $\left(1.25 \mathrm{GPM} / \mathrm{ft}^{2}\right)$. As such, a second filter cleaning routine was performed with $0.5 \mathrm{M}$ oxalic acid on December $29^{\text {th }}, 2008$. For both cleaning events, the procedure used to clean the filters was comparable to that described for Low-Solids Scaling Test \#1 (Section 4.1.1). Execution of the Low-Solids Scaling Test \#2 took place from December $30^{\text {th }}, 2008$, to January $1^{\text {st }}, 2009$. With regard to filter history, the PEP filter bundles had been employed for limited simulant shakedown testing operations, including those described in the preceding sections, before executing Low-Solids Scaling Test \#2.

(a) GB Josephson, OP Bredt, JK Young, and DE Kurath. 2009. Test Plan for Pretreatment Engineering Platform (PEP) Testing (Phase I). TP-RPP-WTP-506, Rev. 0.4, Pacific Northwest National Laboratory, Richland, Washington. 
Table 4.3. Target Operational Conditions for Low-Solids Scaling Test \#2

\begin{tabular}{lc}
\hline \multicolumn{1}{c}{ Parameter } & Target \\
\hline Tank T02A PJM Jet Velocity & $7.3 \pm 0.4 \mathrm{~m} / \mathrm{s}$ \\
Tank T02A PJM Cycle Time & $33 \pm 1 \mathrm{~s}$ \\
Tank T02A PJM Stroke Length & $80 \pm 5 \%$ \\
& $30.3 \pm 1.8 \mathrm{inches}$ \\
Tank T02A Steam Ring Purge Flowrate & $0.10 \pm 0.02 \mathrm{~kg} / \mathrm{min}$ \\
Tank T02A Upper Air Sparger Flowrate & $0.10 \pm 0.02 \mathrm{~kg} / \mathrm{min}$ \\
Tank T02A Total Lower Air Sparger Flowrate & $0.40 \pm 0.05 \mathrm{~kg} / \mathrm{min}$ \\
Number of Filter-Loop Bundles & 5 \\
Filter AV & $15.0 \pm 1.4 \mathrm{ft} / \mathrm{s}$ \\
TMP & $40 \pm 4 \mathrm{psid}$ \\
Slurry Temperature & $25 \pm 2{ }^{\circ} \mathrm{C}$ \\
Maximum Filter Bundle Trans-axial Pressure Drop & $25 \mathrm{psid}$ \\
\hline
\end{tabular}

Table 4.4. A List of Slurry Sampling for Low-Solids Scaling Test \#2

\begin{tabular}{|c|c|c|}
\hline Functional Test Step & Occurrence & Sampling Level \\
\hline $\begin{array}{l}\text { A.1.8-initial 12-hr } \\
\text { filtration }\end{array}$ & $\begin{array}{l}\text { At start of the initial } \\
12 \mathrm{hr} \text { continuous } \\
\text { operation (about } 10 \mathrm{~min} \\
\text { after permeate was } \\
\text { known to be flowing } \\
\text { back into Tank T02A) }\end{array}$ & $\begin{array}{l}\text { Collected one slurry sample for parallel CUF testing from } \\
\text { middle-low CD port. } \\
\text { Collect } 10 \text { samples for physical properties testing from } \\
\text { middle-low CD port. Properties to be measured include slurry } \\
\text { UDS concentration, slurry density, rheology, and particle size. } \\
\text { This report does not present particle-size results. }\end{array}$ \\
\hline $\begin{array}{l}\text { A.1.9-backpulsed } \\
\text { 12-hr filtration }\end{array}$ & $\begin{array}{l}\text { Toward the end of } 12 \text {-hr } \\
\text { backpulsed operations }\end{array}$ & $\begin{array}{l}\text { Collected two samples from the middle-low CD port for } \\
\text { characterization. These samples were analyzed for slurry } \\
\text { UDS concentration and PSD. This report does not present } \\
\text { particle-size results. }\end{array}$ \\
\hline $\begin{array}{l}\text { A.1.10-final } 12-\mathrm{hr} \\
\text { filtration }\end{array}$ & $\begin{array}{l}\text { Toward the end of the } \\
\text { final } 12 \text {-hr continuous } \\
\text { operation }\end{array}$ & $\begin{array}{l}\text { Collected five samples from the midle-low CD sample port } \\
\text { for characterization. These samples were analyzed for slurry } \\
\text { UDS concentration, density, and PSD. This report does not } \\
\text { present particle-size results. }\end{array}$ \\
\hline
\end{tabular}

\subsubsection{CUF Operations for Low-Solids Scaling Test \#2}

Parallel bench-scale filtration tests used the cold-CUF filtration system at APEL. Tests for LowSolids Scaling Test \#2 were conducted in early January 2009. The low-solids simulant slurry collected from the PEP (see sampling for step A.1.8 in Table 4.4) was tested in a 36-hour filter conditioning test that included three equal 12-hour segments of 1) steady state operation, 2) periodic backpulsing, and 3) steady-state operation. As before, the CUF slurry has the same composition as that used for PEP (excluding a slight concentration difference that results from filling the CUF permeate metering system with supernate). The testing proceeded according to these general steps:

1) Load the PEP simulant slurry into the CUF slurry reservoir.

2) Operate the CUF filtration system in recycle mode at target conditions of TMP $=40 \mathrm{psid}$ and $\mathrm{AV}=15 \mathrm{ft} / \mathrm{s}$ for 12 hours. 
3) Operate the CUF filtration system in recycle mode at target conditions of TMP $=40$ psid and $\mathrm{AV}=15 \mathrm{ft} / \mathrm{s}$ and perform a single backpulse every 30 minutes for 12 hours.

4) Operate the CUF filtration system in recycle mode at target conditions of TMP $=40 \mathrm{psid}$ and $\mathrm{AV}=15 \mathrm{ft} / \mathrm{s}$ for 12 hours.

All tests were performed at a target reservoir temperature of $25 \pm 5^{\circ} \mathrm{C}$. Before executing these test steps, the filter was cleaned as described in Section 4.1.2.

\subsection{High-Solids Scaling Test}

The high-solids scaling test assessed the PEP to CUF scaling factor for dewatering operations where the slurry solids concentration approached the limiting gel concentration. For this test, a leached (caustic and oxidative) and washed high-solids concentration (15.4 wt \% UDS) slurry was dewatered until the operational limits of the filtration system were reached. The PEP to CUF scaling factor was then determined by comparing the dewatering curve functionalities (based on the apparent mass transfer coefficient and limiting solids concentration) for CUF and PEP filter flux.

\subsubsection{PEP Operations for High-Solids Scaling}

The high-solids scaling test was performed in March 2009 following Integrated Test B (i.e., the caustic-leach and wash operations in Tank T02A). A mixture of leached and washed simulant slurries from both Integrated Test A and Integrated Test B operations (see Appendix B of TP-RPP-WTP-506 ${ }^{(\mathrm{a})}$ ) was used for the high solids scaling test.

\section{PEP Operations Preceding the High-Solids Scaling Test}

Before Integrated Test B, the filters were cleaned with a 2-M nitric acid solution. Unlike the lowsolids scaling tests (which were executed as part of PEP functional testing), cleaning steps preceding the high-solids scaling test used nitric acid to make filter cleaning prototypic of plant operations. Because of a pump failure, cleaning operations preceding Integrated Test B were conducted in two separate operations. The first cleaning event took place on March $1^{\text {st }}, 2009$. The filters were cleaned with $\sim 200$ gal of $2 \mathrm{M}$ nitric acid using methods comparable to those used in the low solids test. Cleaning was stopped prematurely (i.e., before the first backpulse of the system) because of a leak in the filter-loop pumps after brief contact with nitric acid. Cleaning was resumed on March $7^{\text {th }}, 2009$, and used a diaphragm pump to circulate the 2-M nitric acid cleaning solution through the filtration loop. Integrated Test B was then executed, and no further filter cleaning steps were performed before the high-solids scaling test.

A quantity of the Phase 1 waste simulant slurry sufficient to complete Integrated Test B was prepared in staging tank vessel HLP-VSL-T22. From this source slurry, two separate batches of pre-leach simulant slurry were transferred to Tank T02A, concentrated to $\sim 20-\mathrm{wt} \%$, caustic leached at $98^{\circ} \mathrm{C}$ over a $16-\mathrm{hr}$ period, and subsequently cooled to $60^{\circ} \mathrm{C}$. The pre-leach slurry was concentrated to 20 -wt $\%$ UDS in Tank T02A through periodic batch transfers of slurry from Tank T01A. Concentration of the first batch of simulant employed all five filter bundles, whereas the concentration of the second batch employed only

(a) GB Josephson, OP Bredt, JK Young, and DE Kurath. 2009. Pretreatment Engineering Platform (PEP) Testing (Phase I). TP-RPP-WTP-506, Rev. 0.4, Pacific Northwest National Laboratory, Richland, Washington. 
Filter Bundle 1. It should be noted that before concentration and leaching of the second batch, the first batch was transferred to Tank T01B for storage.

After caustic-leaching, the slurry was concentrated from $\sim 5$-wt $\%$ UDS to $17-w t \%$ UDS using only Filter Bundle 1. To maintain the level in Tank T02A during dewater, periodic batch transfers of causticleached slurry were made from Tank T01B. Next, the caustic leached slurry was washed repeatedly with additions of inhibited process water. Wash solution was continuously recovered by filtering on all five filter bundles. Following post-caustic-leach washing, chromium solids were added to the slurry, and the mixture was concentrated and washed using all five filter bundles. Next, the chromium solids were oxidative-leached by adding permanganate solution $\left(\mathrm{NaMnO}_{4}\right)$ and holding the mixture at $25^{\circ} \mathrm{C}$ for 6 hrs. At the end of the 6-hr hold period, the caustic- and oxidative-leached slurry was again washed with inhibited water. The added wash solution was recovered through filtration on all five filter bundles.

\section{$\underline{\text { High-Solids Scaling Test Operational Test Steps }}$}

The high-solids scaling test was performed from March $20^{\text {th }}$ to March $21^{\text {st }}, 2009$. Test steps associated with the high-solids test were based on Integrated Test A, Steps B.1.22 and B.1.23 (see Table B.1 in TP-WTP-RPP-506). ${ }^{\text {(a) }}$ Apart from the nitric acid rinse before Integrated Test B, no additional filter cleaning steps were performed before the high-solids scaling test. As described above, the filters had been subjected to multiple filtration operations associated with caustic and oxidative leaching and washing steps in Integrated Test B before executing the high-solids test. Prior filter history includes Simulant Shakedown and Integrated Test A and B activities.

To increase the mass of solids for the high-solids scaling tests, the final leached and washed slurry solids from Integrated Test B were combined with a high-solids slurry and a small volume of low-solids supernate left over from Integrated Test A. The high-solids slurry from Integrated Test A had been subjected to a caustic-leaching and washing operation, an oxidative-leaching operation, and a final water wash and concentration. The low-solids supernate corresponded to permeate collected during the leach and wash operations during Integrated Test A.

Combination of Integrated Test A and B high-solids material was accomplished by loading the highsolids slurry from Integrated Test A into Tank T01B (where it was combined with simulant supernate used to rinse the high-solids slurry storage tote) and subsequently transferring the rinsed slurry into Tank T02A where it was mixed with the leached and washed slurry from Integrated Test B.

To prepare for the high-solids test, inhibited water was added to the in-line suction of Pump T42A to dilute the slurry to a specific target starting UDS ( $15 \mathrm{wt} \%$ - see Section 5.3.1). The contents of Tank T02A were then mixed for 10 minutes. Next, the high-solids scaling test was conducted as follows:

1) Filter 1 was aligned, and filters 2 to 5 were bypassed.

2) Heat exchanger UFP-HX-T03A was bypassed, while heat exchanger UFP-HX-T02A was aligned. The filtration loop temperature was controlled to $25^{\circ} \mathrm{C}$.

(a) GB Josephson, OP Bredt, JK Young, and DE Kurath. 2009. Test Plan for Pretreatment Engineering Platform (PEP) Testing (Phase I). TP-RPP-WTP-506, Rev. 0.4, Pacific Northwest National Laboratory, Richland, Washington. 
3) Slurry flow was adjusted to $109 \pm 10 \mathrm{GPM}$ (which translates to a filter AV of $15 \pm 1.4 \mathrm{ft} / \mathrm{s}$ ), and filter TMPs were set to $40 \pm 4$ psid.

4) Permeate flow was aligned to Tank T02A.

5) The permeate valve on filter 1 was opened, and the filter backpulsed five times.

6) The permeate valve was closed, and permeate flow was aligned to collection vessel UFP-VSL-T62B.

7) The permeate valve on filter 1 was opened, and dewatering proceeded until the operational capacity of the PEP circulation loop (in terms of minimum operating volume and/or pump stability limits) was reached.

8) At the end of dewatering, the permeate valve on filter 1 was closed.

The PEP operational conditions associated with the high-solids scaling test are listed in Table 4.5. The actual conditions achieved are discussed in Section 5.3.1. A summary of PEP sampling prescribed for the high-solids scaling test is provided in Table 4.6.

Table 4.5. Target Operational Conditions for High-Solids Scaling Test

\begin{tabular}{lc}
\hline \multicolumn{1}{c}{ Parameter } & Target \\
\hline Tank T02A PJM Jet Velocity & $12 \pm 2 \mathrm{~m} / \mathrm{s}$ \\
Tank T02A PJM Cycle Time & $20 \pm 1 \mathrm{~s}$ \\
Tank T02A PJM Stroke Length & $80-15 \% /+7.5 \%$ \\
& $31.5-6 /+3$ inches \\
Tank T02A Steam Ring Purge Flowrate & Off \\
Tank T02A Upper Air Sparger Flowrate & Off \\
Tank T02A Total Lower Air Sparger Flowrate & Off \\
Number of Filter-Loop Bundles & 1 \\
Filter AV & $15.0 \pm 1.4 \mathrm{ft} / \mathrm{s}$ \\
TMP & $40 \pm 4 \mathrm{psid}$ \\
Slurry Temperature & $25 \pm 2^{\circ} \mathrm{C}$ \\
Maximum Filter Bundle Trans-axial Pressure Drop & $50 \mathrm{psid}$ \\
\hline
\end{tabular}


Table 4.6. A List of Slurry Sampling for High-Solids Scaling Test

\begin{tabular}{|c|c|c|}
\hline Functional Test Step & Occurrence & Sampling Level \\
\hline \multirow[t]{2}{*}{$\begin{array}{l}\text { B.1.22 - Preparation } \\
\text { for the High-Solids } \\
\text { Scaling Test }\end{array}$} & $\begin{array}{l}\text { At the end of the } 10 \text { minute } \\
\text { mixing period following in-line } \\
\text { inhibited water addition. }\end{array}$ & $\begin{array}{l}\text { Collected two samples from the middle-low } \\
\text { CD sample port for characterization of Tank } \\
\text { T02A contents. These samples were analyzed } \\
\text { for slurry UDS concentration and rheology. }\end{array}$ \\
\hline & & $\begin{array}{l}\text { Collected a sample from the middle-low CD } \\
\text { sample port for CUF parallel testing. }\end{array}$ \\
\hline \multirow[t]{3}{*}{$\begin{array}{l}\text { B.1.23 - Execution of } \\
\text { the High Solids } \\
\text { Scaling Test }\end{array}$} & $\begin{array}{l}\text { Immediately before filter } 1 \\
\text { permeate valve was opened }\end{array}$ & $\begin{array}{l}\text { Collected three samples from the middle-low } \\
\text { CD sample port. These samples were } \\
\text { analyzed for slurry UDS concentration. }\end{array}$ \\
\hline & $\begin{array}{l}\text { During dewater (i.e., after filter } 1 \\
\text { permeate valve was opened). }\end{array}$ & $\begin{array}{l}\text { Collected a sample from the middle-low CD } \\
\text { sample port immediately after the permeate } \\
\text { valve on filter } 1 \text { was opened and at } 15 \text { min } \\
\text { intervals thereafter. All samples collected } \\
\text { here were analyzed for slurry UDS } \\
\text { concentration. }\end{array}$ \\
\hline & $\begin{array}{l}\text { End of dewater (i.e., after filter } 1 \\
\text { permeate valve was closed). }\end{array}$ & $\begin{array}{l}\text { Collected three samples from the middle-low } \\
\text { CD sample port. These samples were } \\
\text { analyzed for slurry UDS concentration. }\end{array}$ \\
\hline
\end{tabular}

\subsubsection{CUF Operations for High-Solids Scaling}

Bench-scale testing used the cold-CUF filtration system in APEL. Testing was conducted in late March 2009. The parallel simulant slurry test sample (see sampling for step B.1.22 in Table 4.6 delivered from the PEP was dewatered from its initial concentration to a final concentration of in excess of $20 \mathrm{wt} \%$ UDS in four separate dewatering tests. Each test provided a complete dewatering of the slurry at differing AVs. The permeate collected from each test was added back to the slurry reservoir to reconstitute the slurry for the next test. The high-solids dewatering tests proceeded according to the following general steps:

1) Load the leached/washed solids slurry from PEP into the CUF slurry reservoir. Sample the initial slurry in triplicate.

2) Operate the CUF system in dewatering mode at TMP $=40 \mathrm{psid}$ and $\mathrm{AV}=15 \mathrm{ft} / \mathrm{s}$ and dewatered the slurry to $>20 \mathrm{wt} \%$ UDS or until operating conditions were not sustainable, either due to high axial pressure drop and/or inability to maintain reservoir temperature.

3) Sample concentrated slurry in triplicate.

4) Return all permeate to the CUF slurry reservoir, mixed, and operated the CUF system in dewatering mode at $\mathrm{TMP}=40 \mathrm{psid}$ and $\mathrm{AV}=13 \mathrm{ft} / \mathrm{s}$ and dewatered the slurry to $>20 \mathrm{wt} \%$ UDS or until operating conditions were not sustainable.

5) Return all permeate to the CUF slurry reservoir, mixed, and operated the CUF system in dewatering mode at $\mathrm{TMP}=40 \mathrm{psid}$ and $\mathrm{AV}=17 \mathrm{ft} / \mathrm{s}$ and dewatered the slurry to $>20 \mathrm{wt} \%$ UDS or until operating conditions were not sustainable. 
6) Returned all permeate to the CUF slurry reservoir, mixed, and operated the CUF system in dewatering mode at TMP $=40 \mathrm{psid}$ and $\mathrm{AV}=15 \mathrm{ft} / \mathrm{s}$ and dewatered the slurry to $>20 \mathrm{wt} \% \mathrm{UDS}$ or until operating conditions were not sustainable. This was a repeat of the first dewatering test (step 2).

7) Sampled concentrated slurry and permeate.

All tests were performed at a target reservoir temperature of $25 \pm 5^{\circ} \mathrm{C}$. Apart from minor differences in UDS concentration, the CUF slurry used in testing has the exact same chemical composition as that used in PEP testing. For the current report, only the dewatering test performed at $15 \mathrm{ft} / \mathrm{s}$ (i.e., those associated with steps 2 and 6) are reported. Before executing these test steps, the filter was cleaned as described in Section 4.1.2.

\subsection{Issues Impacting Scaling Tests}

PEP filtration testing was impacted by technical issues related to process instrumentation and data acquisition. Three issues were relevant to filter scale-up testing. These were 1) improper wiring of flow sensor FT-1977, 2) increased uncertainty in flow sensor FT-1977 over that listed by the manufacturer, and 3 ) potential stagnation of fluid in filtration loop thermowells. These three issues are discussed in detail below.

Issue \#1: Improper Wiring of Flow Sensor FT-1977 1976 and mass flow sensor FT-1977 were swapped. As a result, the output for sensor FT-1977, which measures the total lower air sparger flowrate for Tank T02A, was not recorded properly by the DAS. For the current testing, measurement of the total lower air sparger flowrate for Tank T02A was accomplished by using the sparger flowrate reading for FT-1977 read directly from this instrument's digital read-out and subsequently recorded in test instruction associated with the scaling tests. Because the digital reading was available to determine the sensors proper reading, this issue does not impact the current test results. This issue was resolved at a later date by properly rewiring the flow sensor.

Issue \#2: Increased Uncertainty in Flow Sensors FT-1901 and FT-1977 $7^{(\mathrm{b})}$-limited data reported for the upper and lower sparger air flow meters in Tank T02A (FT-1901 and FT-1977, respectively) are subject to increased uncertainty. The flow meter vendor, Micro-Motion, identifies a minimum flowrate $(0.090 \mathrm{~kg} / \mathrm{min})$ where the Coriolis flow uncertainty increases above $0.5 \%$. For the lowest flowrate reported $(0.012 \mathrm{~kg} / \mathrm{min}$ on FT-1977), the estimated uncertainty was $\sim 4 \%$. Since these instruments are used primarily to indicate the approximate air flowrates, higher uncertainty in these data is not considered significant (and does not impact the test results reported herein).

Issue \#3: Potential Stagnation of Fluid in Filtration Loop Thermowells $\mathrm{s}^{(\mathrm{c})}$ - the thermowells associated with select filtration loop temperature sensors did not extend into the process flow as they should. As such, these temperature sensors were isolated from the flowing part of the fluid and were subject to temperature drift resulting from fluid stagnation at high slurry UDS concentration. The affected temperature sensors were TT-0791 (inlet temperature to filter bundle 1), TT-0537 (outlet temperature from filter bundle 5), TT-0513 (outlet temperature for HX-T02A), and TT-0515 (outlet temperature for
(a) NCR 41090.1.
(b) NCR 38767.1.
(c) NCR 42402.1. 
HX-T03A). Because of stagnation concerns with these instruments, no data from these instruments have been used for quality-affecting work. Data from sensors TT-0791, TT-0537, TT-0513, and TT-0515 may be used for qualitative purposes only. Additional discussion about this issue and how it impacts the highsolids scaling test is given in Section 5.3. It should be noted that Issue \#2 forms the basis for selecting temperature sensor TTK-0619 in Tank T02A as the reference temperature sensor for permeate flux temperature corrections. This temperature often falls below that of the filtration loop by several degrees (as a result of mechanical heat input by the pumps). For the current test results, this NCR requires that the prototypic temperature sensor in Tank T02A (TTK-0619) instead of the more appropriate filtration loop temperature sensors TT-0791, TT-0537, TT-0513, and TT-0515. This issue was unfixable. This resolution has consequences for analysis of PEP filtration data because heat-exchange operations intended to remove mechanical heat input from the pumps occurs after filtration. Thus, the permeate temperature is likely several degrees warmer than the slurry temperature indicated by the Tank T02A temperature sensor TTK-0619. In contrast, the CUF is not impacted by a similar error (even though slurry reservoir temperature is used to correct flux) because heat exchange occurs before filtration. As a result, the difference between CUF permeate temperature and the slurry reservoir temperature is likely to be less than the corresponding difference in PEP. The error introduced by the uncertainty in PEP permeate temperatures for filtration introduces errors in the temperature-corrected permeate rate of $\sim 10 \%$ for typical operating temperatures (i.e., $25 \pm 5^{\circ} \mathrm{C}$ ). 



\subsection{Results and Discussion}

The following sections provide a summary of test and process conditions, PEP and CUF scale results, and analytical results for all scaling tests discussed in Section 4.0. Sections 5.1 to 5.3 discuss the processing and filter scaling factor results for Low-Solids Scaling Test \#1, Low-Solids Scaling Test \#2, and the High-Solids Scaling Test. All results reported in this section (including both PEP and CUF data collected by their respective DASs and analytical data) have been processed and reduced. All "raw" PEP data corresponding to the low- and high-solids scaling test, such as PEP permeate production rates (in $\mathrm{kg} / \mathrm{min}$ ) and individual results for analytical sample analyses, can be found in the run report for functional testing ${ }^{(a)}$ in the run report for Integrated Test $\mathrm{A},{ }^{(\mathrm{b})}$ and in the various raw data reports generated by PNNL and offsite analytical vendors. A document presenting raw (unanalyzed) CUF data is not available.

\subsection{Test Results for Low-Solids Scaling Test \#1}

PEP operations for Low-Solids Scaling Test \#1 were conducted from $1517 \mathrm{hrs}$ November $23^{\text {rd }}, 2008$, to 0439 hrs November $25^{\text {th }}, 2008$, Pacific Standard Time (PST). PEP process data were recorded by the PEP DAS at a frequency of $1 \mathrm{~Hz}$. For subsequent analyses, all relevant $1 \mathrm{~Hz}$ data (i.e., those over the process times of interest) were pulled and averaged over 1-minute intervals. The sample for laboratoryscale (cold-CUF) testing (ID\# S 000FL 008 XX 0250 CUF 4) was taken at 1601 hrs November $23^{\text {rd }}$, 2008, from the middle/low Coriolis densitometer port in Tank T02A. This sample was delivered to APEL for bench-scale testing. It should be noted that cold-CUF sample collection occurs after the start of filtration operations (and as such, the slurry corresponds directly to that tested in PEP and is not affected by differences in concentration that could result if the sample has been pulled before filling the permeate collection/metering system with permeate). Cold-CUF operations were conducted from $0653 \mathrm{hrs}$ December $2^{\text {nd }}, 2008$, to $1902 \mathrm{hrs}$ December $3^{\text {rd }}, 2008$, PST. The CUF DACS recorded CUF data at a frequency of $0.4 \mathrm{~Hz}$. The CUF DACS then averaged all $0.4-\mathrm{Hz}$ data over 1-minute process intervals. For all CUF data analyses, the 1-minute data averages were employed. The test operations and scaling results for Low-Solids Scaling Test \#1 are discussed in detail in the following sections.

\subsubsection{PEP Operations}

For PEP operations associated with Low-Solids Scaling Test \#1, the circulating mass of slurry in the filtration loop and Tank T02A had a combined mass of approximately $1200 \mathrm{~kg}$ of a $6.9-\mathrm{wt} \%$ UDS slurry (based on estimates and analysis of tank levels and transfer volumes). This yielded an undissolved slurry solids-to-filter area ratio of $1.1 \mathrm{~kg} / \mathrm{ft}^{2}$. This range is similar to, but slightly lower, than the low-end of the range of slurry solids-to-surface area ranges planned for use in WTP, which is estimated to be approximately 1.7 to $16 \mathrm{~kg} / \mathrm{ft}^{2}$. ${ }^{\text {(c) }}$ While the current ratio of $1.1 \mathrm{~kg} / \mathrm{ft}^{2}$ employed for the low-solids test is lower than anticipated for use in WTP; previous scaling studies in WTP-RPT-168, Rev. 0, suggest that a difference of this magnitude is not expected to impact filtration scaling substantially (see Section 3.4.8). It should also be noted that the estimated solids-to-surface area ratio of $1.1 \mathrm{~kg} / \mathrm{ft}^{2}$ for PEP Low-Solids

(a) PEP Run Report for Simulant Shakedown/Functional Testing, WTP-RPT-190.

(b) PEP Run Report for Integrated Test A; Caustic Leaching in UFP-VSL-T01A, Oxidative Leaching in UFP-VSLT02A, WTP-RPT-191.

(c) The given range of 1.7 to $16 \mathrm{~kg} / \mathrm{ft}^{2}$ is based on leaching scenarios outlined in Sections 2.3.4.1.1 and 2.3.4.1.2 in 24590-WTP-RPT-PT-02-005, Rev 4. 
Scaling Test \#1 is lower than employed in the parallel CUF Low-Solids Test \#1 $\left(1.5 \mathrm{~kg} / \mathrm{ft}^{2}\right)$ and for both PEP and CUF tests associated with the Low-Solids Scaling Test \#2 $\left(1.4 \mathrm{~kg} / \mathrm{ft}^{2}\right)$-see Section 5.2 .

However, based on the arguments made with respect to differences in PEP and WTP solids-to-filter area ratios, the difference in PEP and CUF solids-to-area ratios for Low-Solids Scaling Test \#1 is not expected to impact filtration scaling calculations substantially (see discussion in Section 3.4.8). Overall, the lowsolids scaling test results should be representative of the lower bound of slurry solids-to-filter surface area in the PTF.

The physical properties of the PEP slurry are listed in Table 5.1. The physical properties, particularly density, dissolved solids, and permeate viscosity, are much lower than expected for the as-prepared simulant and suggest an inadvertent dilution of the slurry supernate phase with a low-dissolved solids medium (such as inhibited water) before Low-Solids Test \#1. Table 5.2 shows the operational parameters for Tank T02A PJM sparging systems that were achieved in Low-Solids Scaling Test \#1. Table 5.2 indicates that most target conditions were met (within acceptable tolerances) with the exception of the average Tank T02A PJM jet velocity. The lower-than-targeted PJM jet velocity may mean that mixing in Tank T02A is less-than-prototypic/expected.

Table 5.1. PEP Slurry Properties for Low-Solids Scaling Test $\# 1^{(\mathrm{a})}$

\begin{tabular}{lcl}
\hline Property & Measured Value & $\mathrm{Units}$ \\
\hline Approximate Slurry Mass Tested & 1200 & $\mathrm{~kg}$ \\
Slurry Solids-to-Filter Area Ratio & 1.1 & $\mathrm{~kg} / \mathrm{ft}^{2}$ \\
Rheology & $0.052 \pm 0.002$ & $\mathrm{~Pa}$ \\
$\quad$ Bingham Yield Stress & $4.34 \pm 0.04$ & $\mathrm{mPa} \cdot \mathrm{s}$ \\
$\quad$ Bingham Consistency & 2.4 & $\mathrm{mPa} \cdot \mathrm{s}$ \\
$\quad$ Supernate Viscosity & $1.25 \pm 0.02$ & $\mathrm{~kg} / \mathrm{L}$ \\
Density & $1.18 \pm 0.03$ & $\mathrm{~kg} / \mathrm{L}$ \\
$\quad$ Slurry Bulk Density & & \\
Permeate Density & $30.4 \pm 0.6$ & $\mathrm{wt} \%$ \\
Solids Concentrations & $6.9 \pm 0.3$ & $\mathrm{wt} \%$ \\
$\quad$ Total Solids & $25.3 \pm 0.6$ & $\mathrm{wt} \%$ \\
Undissolved Solids & $36.3 \pm 2.3$ & $\mathrm{wt} \%$ \\
$\quad$ Dissolved Solids In Permeate & & \\
Centrifuged Solids & &
\end{tabular}

(a) Reported uncertainties represent two sample standard deviations. In some cases, there were insufficient measurements to determine uncertainty.

Table 5.2. Operation Conditions Achieved for Tank T02A PJM and Sparging Systems During LowSolids-Scaling Test \#1

\begin{tabular}{lcc}
\hline Parameter & Target & Actual \\
\hline Tank T02A PJM Jet Velocity & $7.3 \pm 0.4 \mathrm{~m} / \mathrm{s}$ & $5.9 \mathrm{~m} / \mathrm{s}$ \\
Tank T02A PJM Cycle Time & $33 \pm 1 \mathrm{~s}$ & $33.2 \mathrm{~s}$ \\
Tank T02A PJM Stroke Length & $30.3 \pm 1.8 \mathrm{in}$ & $31 \mathrm{in} .(82 \%)$ \\
Tank T02A Steam Ring Purge Flowrate & $0.10 \pm 0.02 \mathrm{~kg} / \mathrm{min}$ & $0.10 \mathrm{~kg} / \mathrm{min}$ \\
Tank T02A Upper Air Sparger Flowrate & $0.10 \pm 0.02 \mathrm{~kg} / \mathrm{min}$ & $0.10 \mathrm{~kg} / \mathrm{min}$ \\
Tank T02A Total Lower Air Sparger Flowrate & $0.40 \pm 0.05 \mathrm{~kg} / \mathrm{min}$ & $0.40 \mathrm{~kg} / \mathrm{min}$ \\
Number of Filter-Loop Bundles & 5 & 5 \\
\hline
\end{tabular}


The PEP process measurements and sample analyses for Low-Solids Scaling Test \#1 are shown in Figure 5.1 to Figure 5.6. Figure 5.1 shows the AV versus elapsed test time (referred to as test time hereafter). Although there was some initial drift in the velocity measured at the suction to Pump T42A, AVs were relatively constant beyond the first 6 hrs of testing. The increased scatter in AV during the backpulsed operations evident in Figure 5.1 is a result of backpulsing operations. Overall, average filter $\mathrm{AVs}$ of $15.3 \mathrm{ft} / \mathrm{s}$ and $14.8 \mathrm{ft} / \mathrm{s}$ were achieved during testing and fell within the specified range of $15.0 \pm$ $1.4 \mathrm{ft} / \mathrm{s}$.

It should be noted that the initial drift during the first $6 \mathrm{hr}$ of testing is probably associated with the development of a steady state level of entrained air in the slurry. In addition, the different readings from the two flow meters can be attributed to entrained air (bubbles are compressed by the pump head pressure and the apparent fluid velocity decreases). Because the pressure experienced by the slurry in the filtration loop falls between the low-pressure bound at the suction to pump T42A and the high-pressure bound at the discharge to pump T43A, the AVs derived from flow meters FT-0623 and FT-0635 should bound the AVs that exist along the filter bundles.

Figure 5.2 shows the TMPs achieved for Filters 1 through 5 during Low-Solids Scaling Test \#1. With exception of the approach to steady TMP conditions observed during the first 15 to 30 minutes of testing, filter TMPs were relatively stable throughout testing. The scatter observed during backpulsing operations result from inclusion of backpressures in the TMP data. During continuous operations, the average TMP for all filters fell within \pm 0.5 psid of the target of 40 psid. It should be noted that the measured TMP is used to correct filter fluxes to a standard TMP of 40 psid.

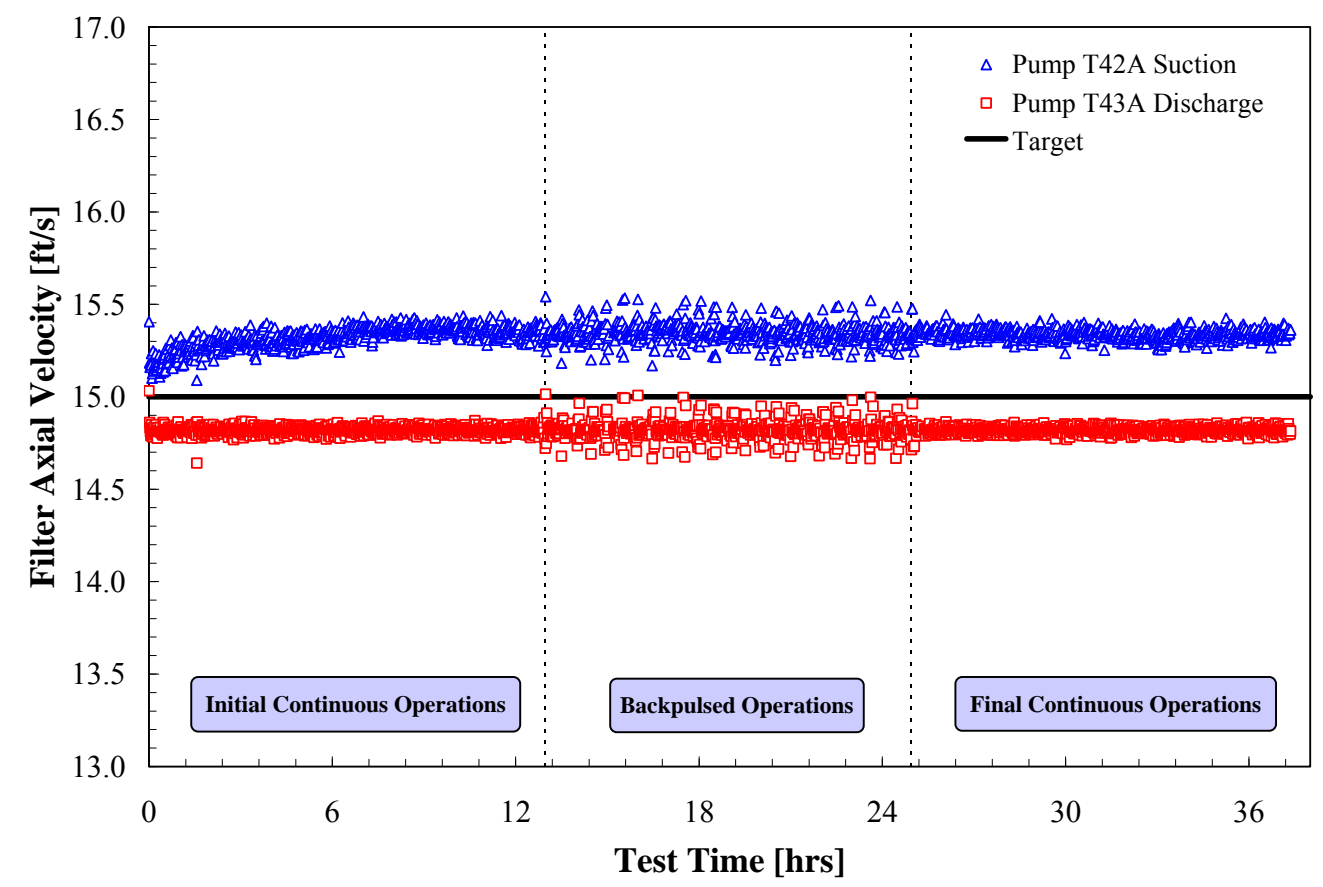

Figure 5.1. Filter AVs Achieved at PEP During Low-Solids Scaling Test \#1. The velocities at Pump T42A suction and Pump T43A discharge are based on sensors FT-0623 and FT-0635, respectively. The target velocity was $15.0 \pm 1.4 \mathrm{ft} / \mathrm{s}$. Average velocities of $15.3 \mathrm{ft} / \mathrm{s}$ and 14.8 (at the suction and discharge to the pumps, respectively) were achieved. 


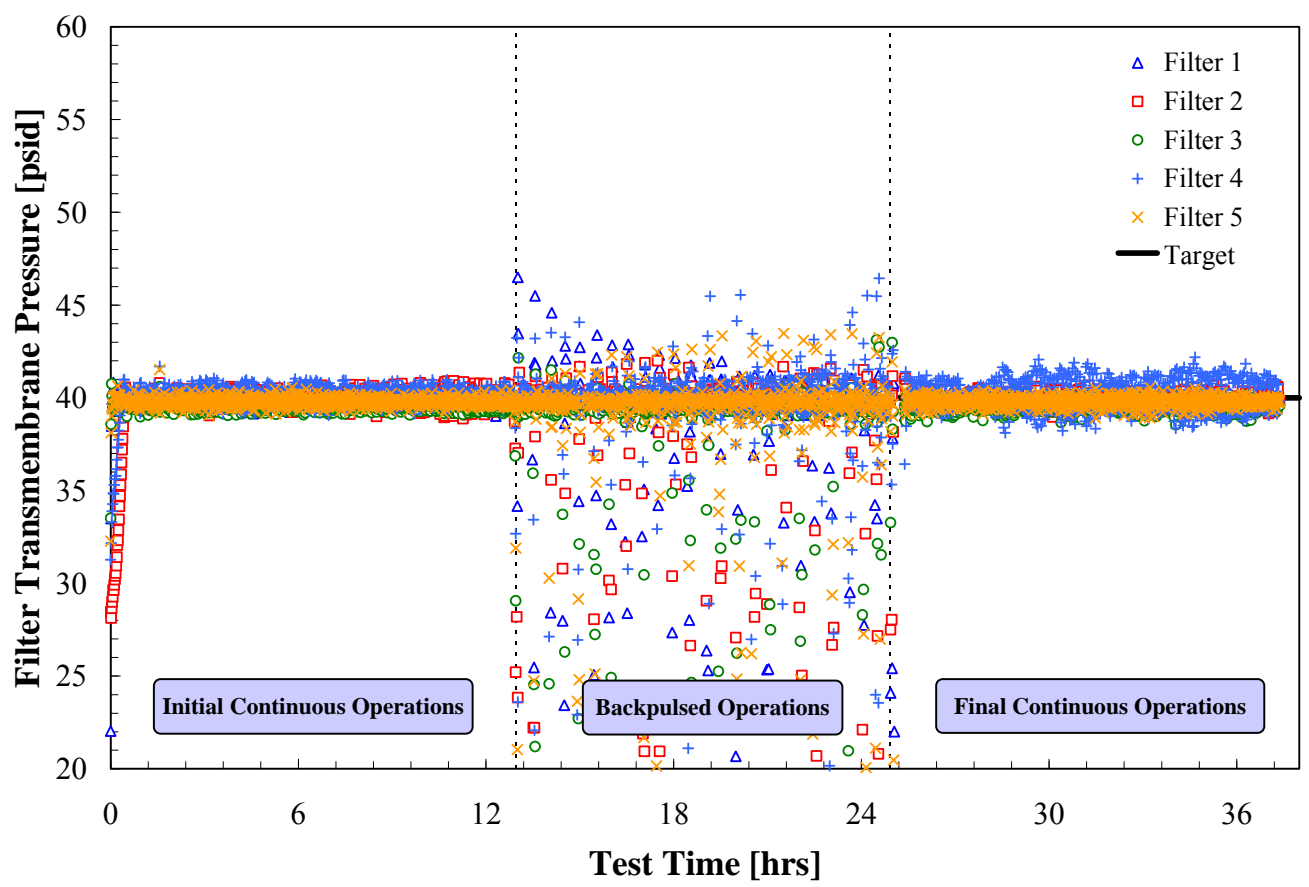

Figure 5.2. Filter TMPs Achieved at PEP During Low-Solids Scaling Test \#1. The target TMP for all filters was 40 psid. The average TMP for all filters fell with \pm 0.5 psid of this target.

The APDs across each of the five filter bundles during Low-Solids Scaling Test \#1 are shown in Figure 5.3. The upper limit of 25 psid was not exceeded at any time during the test. As expected, the 10-ft filter bundles (i.e., Filters 1-3) had higher APD than the $8 \mathrm{ft}$ filter bundles (i.e., Filters 4 and 5).

Figure 5.4 shows the temperature profile for Tank T02A. This temperature is used to correct measured filter fluxes for all bundles to a standard test temperature of $25^{\circ} \mathrm{C}$. The average test temperature for Tank T02A was $26.5^{\circ} \mathrm{C}$ and was within the specified target range of $25 \pm 2{ }^{\circ} \mathrm{C}$. However, as stated in previous sections, the filter flux temperature corrections use Tank T02A vessel temperature because of concerns of potential stagnation of slurry in the filtration loop thermowells (see Section 4.4). The filtration loop temperature is likely several degrees higher as a result of mechanical heat input by the pumps, and as a result, the filtration loop temperature may exceed the specified range of $25 \pm 2^{\circ} \mathrm{C}$. This difference introduces uncertainty in the correction of PEP filtration data relative to that of CUF (see Section 4.4). 


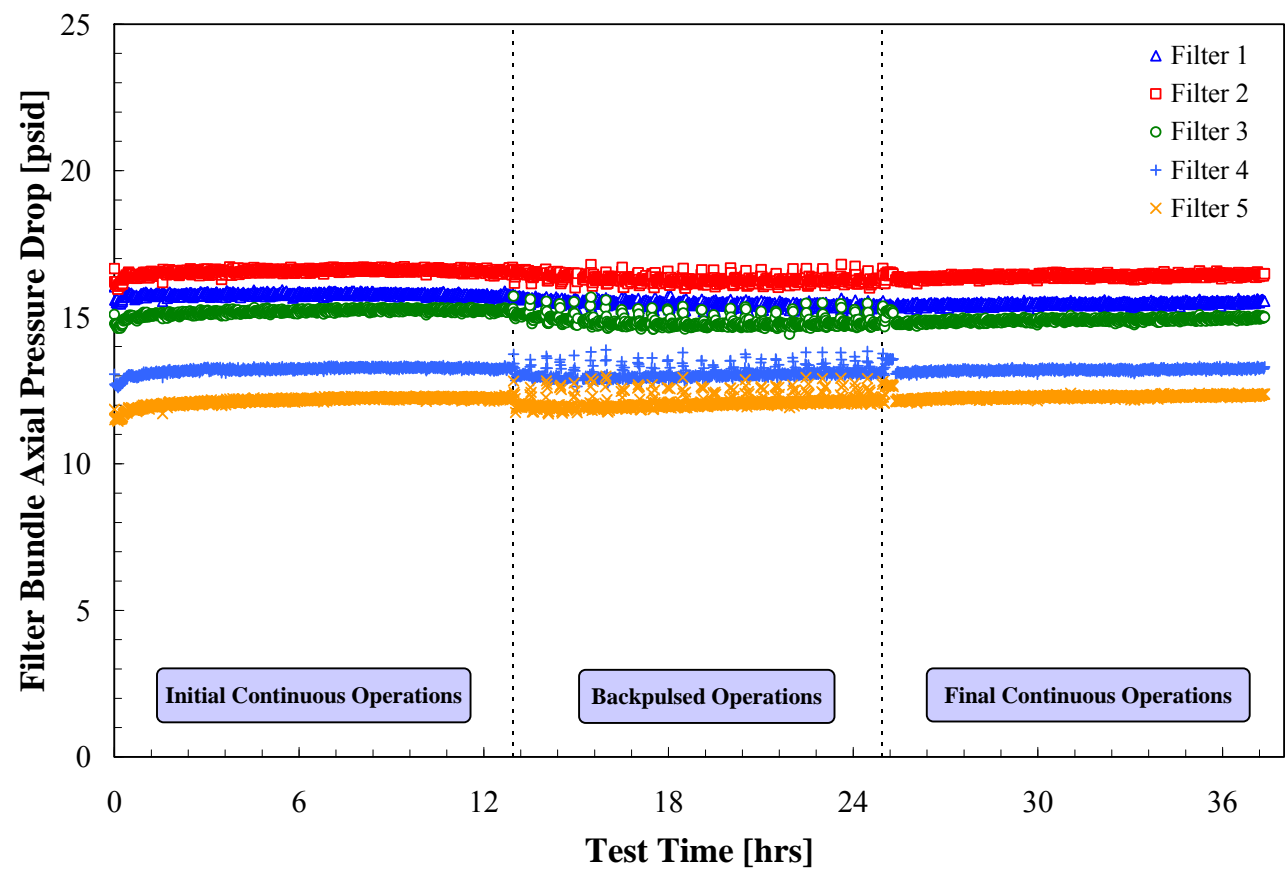

Figure 5.3. Filter APDs Observed at PEP During Low-Solids Scaling Test \#1. The upper allowable limit for APD was 25 psid for all filter.

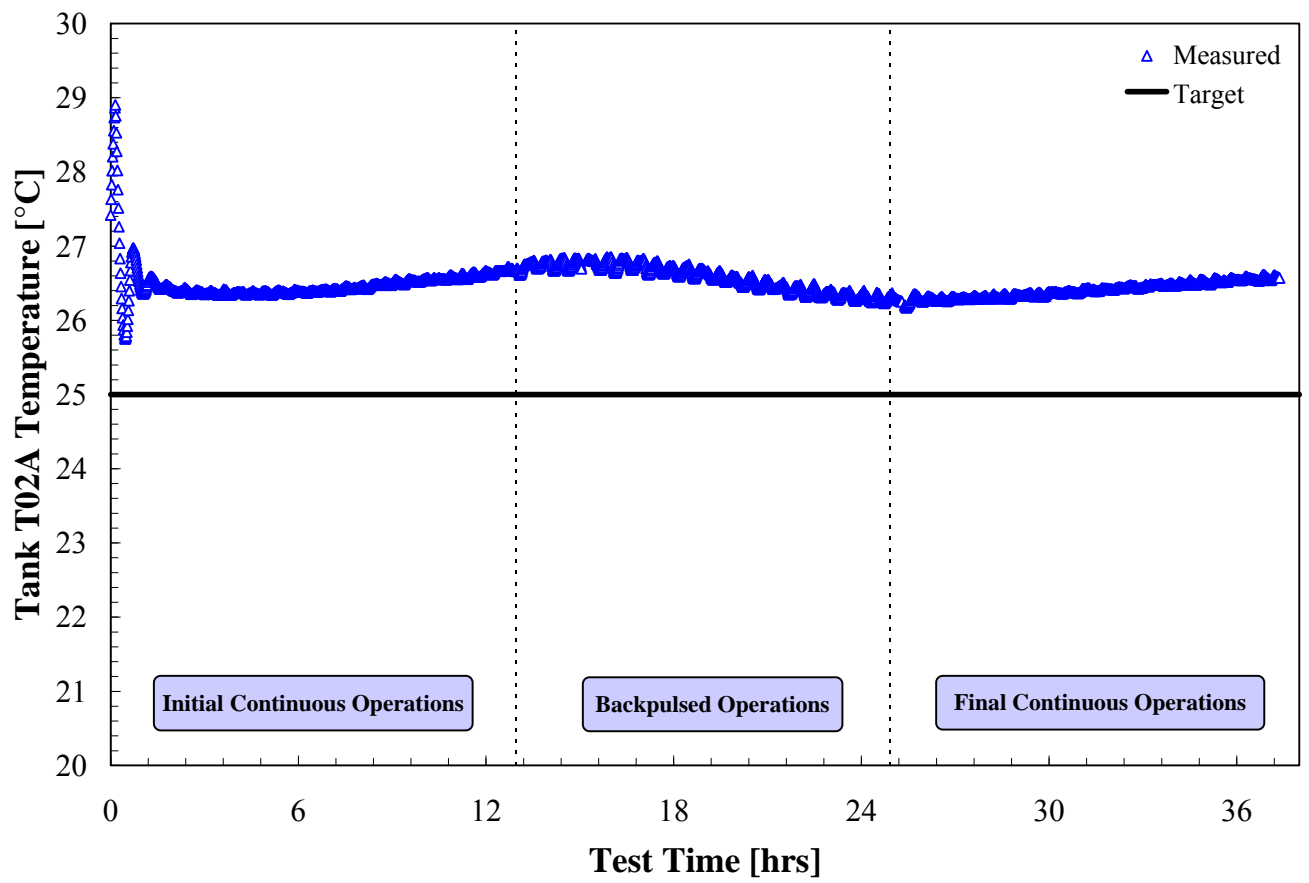

Figure 5.4. Temperature of Vessel UFP-VSL-T02A During Low-Solids Scaling Test \#1. The target temperature of the filtration loop during testing was $25^{\circ} \mathrm{C}$. The average temperature for Tank T02A was $26.5^{\circ} \mathrm{C}$. 
Figure 5.5 shows the temperature and TMP-corrected filter flux measured during Low-Solids Scaling Test \#1 for each of the five filter bundles. During the initial 12-hr period of continuous (non-backpulsed) operations, the filter fluxes for all five filter bundles are similar. The flux of each filter monotonically decreases from $\sim 0.10 \mathrm{GPM} / \mathrm{ft}^{2}$ to $\sim 0.04 \mathrm{GPM} / \mathrm{ft}^{2}$ during the first test segment. The decline is consistent with increased resistance of filter permeate from solids cake formation and filter depth fouling.

It should be noted that in contrast to similar filter conditioning tests done in Russell et al. (2009c), the PEP filter bundles were not backpulsed at the start of testing. The flux recovery associated with this backpulse recovery has been used in previous CUF filter conditioning studies to identify the "start" (i.e., zero time) of filtration as it corresponds to a filter condition relatively free of a solids cake and depth fouling (assuming the filter was cleaned before testing). Because an initial backpulse of the filters was not done in the current test, the start of filtration must be identified using another basis. As such, the current studies (i.e., both Low-Solids Tests \#1 and \#2 for PEP) identify the start of filtration using the initial permeate rate maximum observed immediately after permeate production is started. Selection was also constrained by the requirements that the target TMP and AV had been reached and that the pulse pots had been filled with permeate. One consequence of this definition for Low-Solids Scaling Test \#1 is that the filters were run $\sim 1 \mathrm{hr}$ longer than the 12-hr period called for relative to the identified start of filtration.

The initial backpulse done at the start of backpulsing operations appears to restore flux ${ }^{(a)}$ across all five bundles to approximately $0.07 \mathrm{GPM} / \mathrm{ft}^{2}$. However, continued backpulsing at 30 -minute intervals appears to cause a divergence in the filter flux achievable in each filter bundle. Specifically, repeated backpulsing of filter bundles 1 and 2 does not substantially change the filter flux - the recovered flux remains relatively constant at $0.07 \mathrm{GPM} / \mathrm{ft}^{2}$. In contrast, repeated backpulsing of filters 3 through 5 causes a significant decline in the recovered filter flux over time. Filter bundle 3 shows moderate decline, and filter bundles 4 and 5 show severe decline (with bundle 4 appearing to be slightly more affected than 5 in terms of the magnitude of recovered flux). The decline is characterized by the reduced capability of the system backpulsing to restore filter flux. As the result, the divergence in the recovered flux between the five filter bundles persists into the final $12 \mathrm{hrs}$ of testing (i.e., the fluxes do not re-converge to flux levels observed in the first $12-\mathrm{hr}$ period of operation).

(a) It should be noted that the flux recovered after backpulsing in Figure 5.5 is moderated somewhat by averaging of flux data over 1-minute intervals. As a result, the non-averaged flux likely varies more widely (and can reach higher flux magnitudes for short periods of time) than reported herein. 


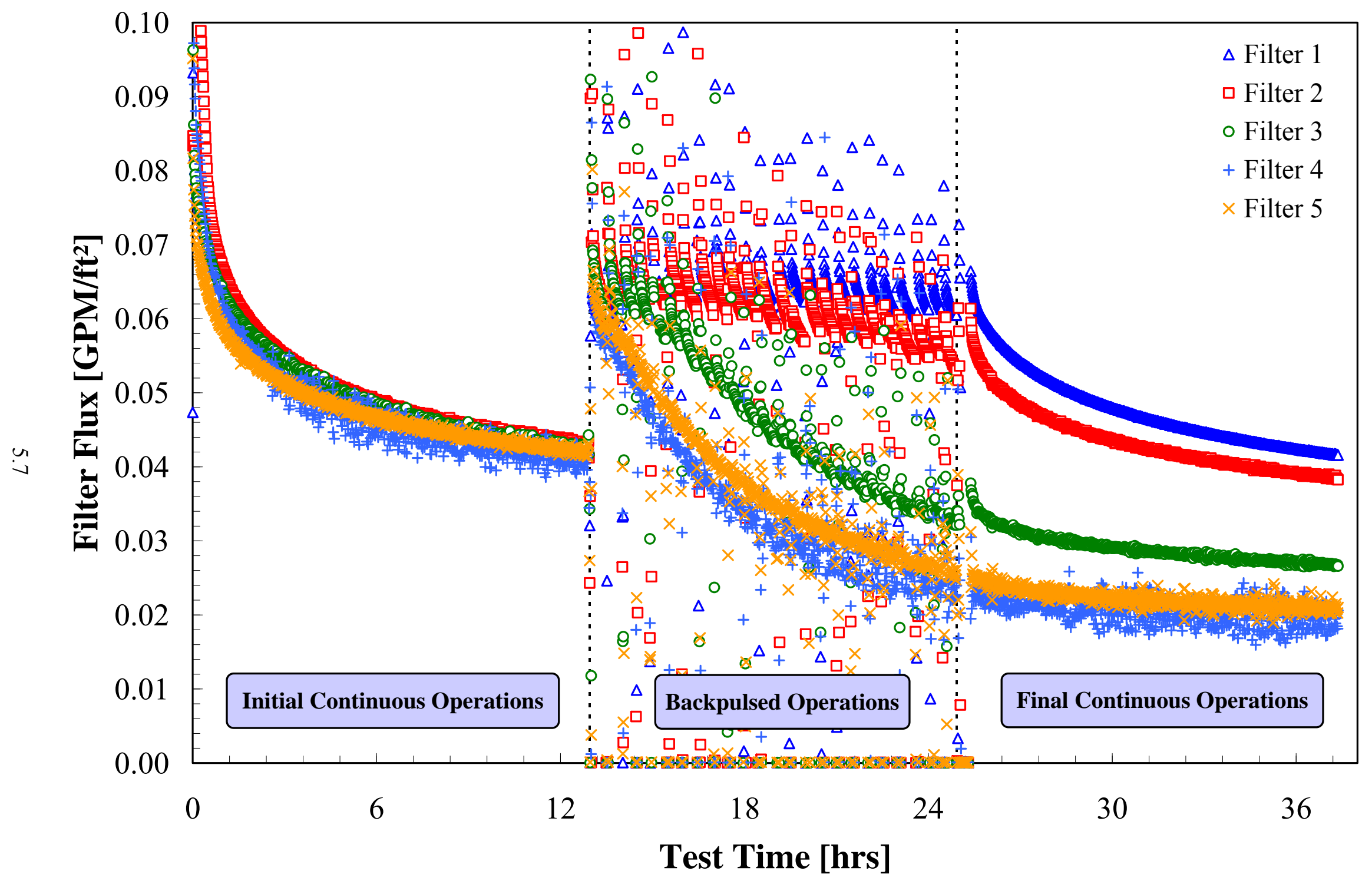

Figure 5.5. Individual Permeate Flux for PEP Filters (corrected for variation in TMP and temperature) During Low-Solids Scaling Test \#1 
The magnitude of filter flux observed for individual filters during the final 12-hr continuous (nonbackpulsed) segment was strongly influenced by the flux divergence that occurred during backpulsing operations. While the differences in flux caused by divergence persist, they do not appear to change dramatically during the final 12-hr period of testing (i.e., the individual fluxes appear to be tending toward a stable steady-state value, although that is not reached over the course of testing). More specifically, the fluxes for filters 1 and 2 remain high (relative to the other filters) and are relatively similar to one another. Likewise, the fluxes for filters 4 and 5 remain low (relative to filters 1 and 2) and relatively similar to one another. The flux for filter bundle 3 falls between these two extremes. With regard to filter flux transience, filters 1 and 2 show a decline consistent with filter cake formation (and possibly depth fouling). For both bundles, the flux decreases from $0.07 \mathrm{GPM} / \mathrm{ft}^{2}$ to $0.04 \mathrm{GPM} / \mathrm{ft}^{2}$. Transience is much less dramatic for filter 3; filter flux starts at $0.04 \mathrm{GPM} / \mathrm{ft}^{2}$ and ends slightly below $0.03 \mathrm{GPM} / \mathrm{ft}^{2}$. Filter fluxes for filters 4 and 5 do not change substantially - both range from $0.03 \mathrm{GPM} / \mathrm{ft}^{2}$ to $0.02 \mathrm{GPM} / \mathrm{ft}^{2}$. This is typical for filters affected by strong depth fouling - the loss of flux from depth fouling should reduce both the rate and degree of cake formation.

As will be discussed in Section 5.2, the flux divergence observed in Low-Solids Scaling Test \#1 during backpulsed operations is reproducible. Indeed, similar divergence is observed in Low-Solids Scaling Test \#2. The mechanism for flux divergence across filter bundles in the low-solids scaling tests is not currently understood. The three primary characteristics of the divergence are:

- Significant (noticeable) flux divergence appears to only occur during backpulsed operation of the filter bundles.

- Only the performance of the downstream filter bundles are impacted during the course of the current test.

- For affected filters, backpulsing does not restore the loss in filter flux-any recovery is typical of that associated with cake disruption (see filter bundle 3 in Figure 5.5).

Based on these observations, it can be speculated that the divergence results from irreversible depth fouling of the porous filter element with fine particulate slurry solids. This depth fouling occurs shortly after each backpulse during the interim period between filter cake disruption and reformation when the filter surface is exposed. For a single backpulse (like those associated with the initial and filter test periods in Figure 5.5), the degree of depth fouling may not be significant enough to observe. However, given repeated backpulsing (such as that done in the second period of operation), the incremental impact of depth fouling becomes apparent. Under this mechanism, depth fouling would not occur during continuous non-backpulsed operations because the filter cake forms a protective surface, which reduces the opportunity for fines to reach the porous filter membrane.

While the depth fouling mechanism outlined in the preceding paragraph explains flux decline for individual filters, it does not explain why only the downstream filters would be affected. Indeed, only filter bundles 3 to 5 were significantly affected by the flux reduction phenomenon. It can be speculated that the downstream filters are observing "downstream" fines that have been collected on and subsequently released from (as a result of backpulsing) upstream filter bundles. Such a mechanism would be believable if the slurry residence time in the filtration loop were near to or greater than the period of time between the backpulsing of each bundle. Typically, filter bundles 1,2, and 3 were backpulsed within a few seconds of each other, and then filters 4 and 5 were backpulsed as soon as the pulse pots refilled (estimated to be 2 to 5 minutes after the backpulse of filters 1 to 3 ). As such, the time between backpulsing of the upstream bundles and downstream bundles ( $\sim 2$ to 5 minutes) is much larger than the 
residence time of the slurry in the filter loop ( $\sim 45$ seconds). This means that fines released from upstream filter bundle cake disruption should have sufficient time to adequately disperse through the loop and Tank T02A before backpulsing of the downstream filters. If an immediately downwind effect were the cause of flux divergence, then it is expected that filter 3 (which is immediately downstream of two backpulsed filters and is disrupted at the same time as the upstream filters) would be the most likely candidate for strong fouling. As shown by the test results, this is not the case.

Another potential mechanism for flux divergence relies on fines depletion. That is, fines capable of fouling the filter elements could become trapped in the filter cake during continuous backpulse operations. In this case, ordered backpulsing of the filters (with the upstream filters being backpulsed first) would release these fines back into the slurry facilitating filter fouling. However, because of the residence time, the fines would be available for fouling only after they are remixed into the slurry in Tank T02A. If the time required to remix the fines ( 1 to 2 minutes) is less than the time it takes to reform a protective cake layer, then the upstream filters would not foul. In contrast, downstream filters would be backpulsed just as the fines completed mixing and were returned to the filter loop. As such, the remixed fines would have access to the downstream filter surface and porous substructure during backpulsing, facilitating significant fouling of the downstream filters.

Thus, several mechanisms can be proposed to explain the divergence of filter flux during backpulsed operations in the low-solids scaling test. The current PEP and/or CUF filter flux data reported herein are not sufficient to identify and validate which of these proposed mechanisms (if any) are correct. Additional testing beyond the scope of the current study is required to further identify, revise, and/or validate the mechanisms for the filter flux divergence. As will be discussed in later sections, this divergence limits application of scaling factors derived for the low-solids scaling tests.

Finally, the total PEP filter flux (i.e., the area average of all five filter bundle fluxes) is shown in Figure 5.6. The initial 12-hr period of continuous operation with no backpulsing was characterized by a monotonic flux decline governed by filter cake formation (and possibly slow depth fouling). Flux in this first segment varies from $0.10 \mathrm{GPM} / \mathrm{ft}^{2}$ to $0.04 \mathrm{GPM} / \mathrm{ft}^{2}$. The flux during the period with backpulsing operation shows a significant irreversible flux decline driven by what is suspected to be depth fouling in the downstream filter bundles. The flux in this second period of operations, which would nominally be constant (given no depth fouling), declines from approximately $0.07 \mathrm{GPM} / \mathrm{ft}^{2}$ to $0.04 \mathrm{GPM} / \mathrm{ft}^{2}$. In the final 12-hr period of continuous operation with no backpulsing, the flux shows monotonic decline in flux from $0.045 \mathrm{GPM} / \mathrm{ft}^{2}$ to $0.030 \mathrm{GPM} / \mathrm{ft}^{2}$. Relative to the initial 12 -hr period of operation, the final period of non-backpulsed operations showed a lower flux magnitude and a slower flux decline. 


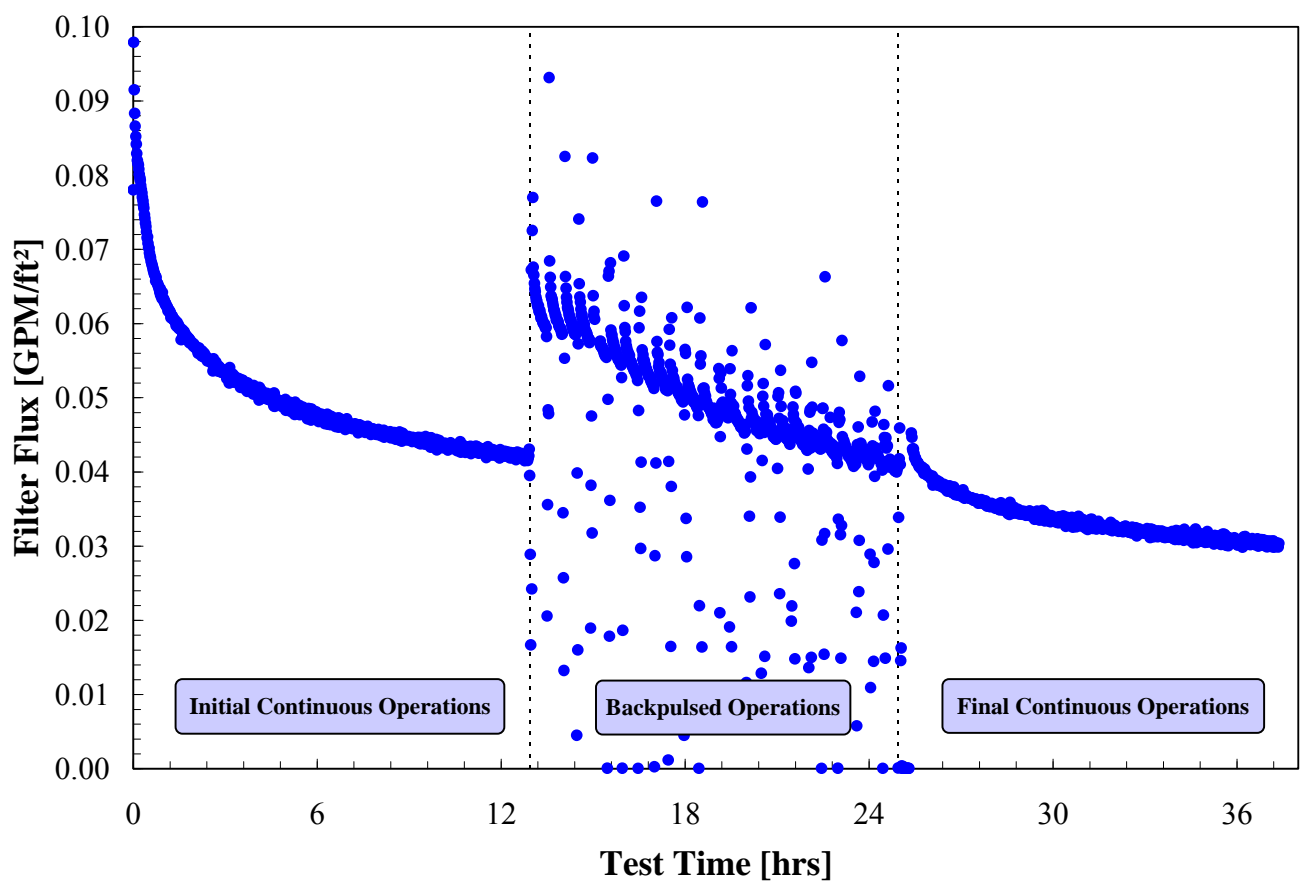

Figure 5.6. Total Permeate Flux for PEP filters (normalized for variation in TMP and temperature) During Low-Solids Scaling Test \#1

It should be noted that the flux transience observed for the individual filter bundles as well as the total filter flux appeared to continue over the entire duration of filter flux testing. Although the rate of filter flux decline slows throughout the test, it appeared that a steady-state filter flux was not entirely reached at the end of 12-hr periods of continued operations. As such, it is not possible to assess the existence or magnitude of a "fully conditioned" steady-state filter flux from the current tests. Additional testing that examines much longer periods of filtration is required. Because steady state was not achieved in the current testing, all scaling factor results derived from Low-Solids Scaling Test \#1 are subject to variation from filter transience.

\subsubsection{CUF Operations}

The waste simulant slurry for parallel CUF testing was received (sample was taken before the start of the PEP test) from the PEP in late November 2008. The slurry was loaded into the slurry reservoir on December 2, 2008. The low-solids filter conditioning test proceeded according to the steps listed in Section 4.1.2. Table 5.3 summarizes the CUF test conditions. For the test, $5.805 \mathrm{~kg}$ of a $6.9 \mathrm{wt} \%$ UDS waste simulant slurry was added to the cold-CUF system. This yielded a slurry solids-to-filter surface area ratio of $1.5 \mathrm{~kg} / \mathrm{ft}^{2}$. As discussed in Section 5.1.1, this ratio of $1.5 \mathrm{~kg} / \mathrm{ft}^{2}$ is higher than that employed for parallel PEP testing (which was $1.1 \mathrm{~kg} / \mathrm{ft}^{2}$ ) but is slightly lower than planned for use in WTP (1.7 to $\left.16 \mathrm{~kg} / \mathrm{ft}^{2}\right)$. However, previous scaling studies in WTP-RPT-168, Rev. 0, suggest that this difference in ratios (i.e., 1.1 versus $15 \mathrm{~kg} / \mathrm{ft}^{2}$ ) is not expected to impact filtration scaling substantially (Daniel et al. 2009). 
Table 5.3. Test Conditions and Operational Parameters for CUF Low-Solids Scaling Test \#1

\begin{tabular}{ll}
\hline \multicolumn{1}{c}{ Parameter } & \multicolumn{1}{c}{ Value } \\
\hline Mass of slurry added to reservoir: & $5.81 \mathrm{~kg}$ \\
Ratio of slurry solids-to-filter surface area: & $1.5 \mathrm{~kg} / \mathrm{ft}^{2}$ \\
Process start time: & $12 / 02 / 2008$ 06:53 PST \\
Process end time: & $12 / 03 / 2008$ 19:00 PST \\
Elapsed time (duration): & 36.12 hours \\
Mixer impeller configuration & Two impellers: a) 2-inch diameter propeller at the end of \\
& $\begin{array}{l}\text { the shaft at one tank radius from the bottom b) 3-inch } \\
\text { diameter, pitched, 3-blade turbine positioned } 5 \text { inches }\end{array}$ \\
& above the propeller \\
Mixer speed & 450 rpm \\
\hline
\end{tabular}

Once the initial target conditions of TMP $=40 \mathrm{psid}$ and AV $=15 \mathrm{ft} / \mathrm{s}$ were established for this test, the filtration system required only minor adjustments in operational parameters during the completion of the three segments of the conditioning test. System operational parameters, including AV, TMP, APD, and slurry reservoir temperature, are shown in Figure 5.7 to Figure 5.10.

Figure 5.7 and Figure 5.8 show the filter AV and TMP, respectively, for CUF Low-Solids Scaling Test \#1. Both were maintained at relatively constant levels throughout the testing. An average AV of $14.9 \pm 0.7 \mathrm{ft} / \mathrm{s}(\mu \pm 2 \sigma)^{(\mathrm{a})}$ was achieved relative to a target of $15 \mathrm{ft} / \mathrm{s}$. Likewise, an average TMP of $40.2 \pm 0.8$ psid $(\mu \pm 2 \sigma)$ was achieved against a TMP target of 40 psid. Figure 5.9 shows the filter APD for CUF operations associated with Low-Solids Scaling Test \#1. The APD is subject to significant scatter (because of process noise at the filter inlet) and varies between 1.5 and 3.5 psid. Although APD appears to show a slight increase during the period of testing, it is difficult to assess the significance of this increase relative to the APD signal scatter. Finally, Figure 5.10 shows the CUF slurry reservoir temperature during the test. The average test temperature was $24.6 \pm 0.5^{\circ} \mathrm{C}(\mu \pm 2 \sigma)$; the target test temperature was $25^{\circ} \mathrm{C}$.

(a) This notation indicates that the reported value is the mean $(\mu)$, and the uncertainty is twice the sample standard deviation $(\sigma)$. 


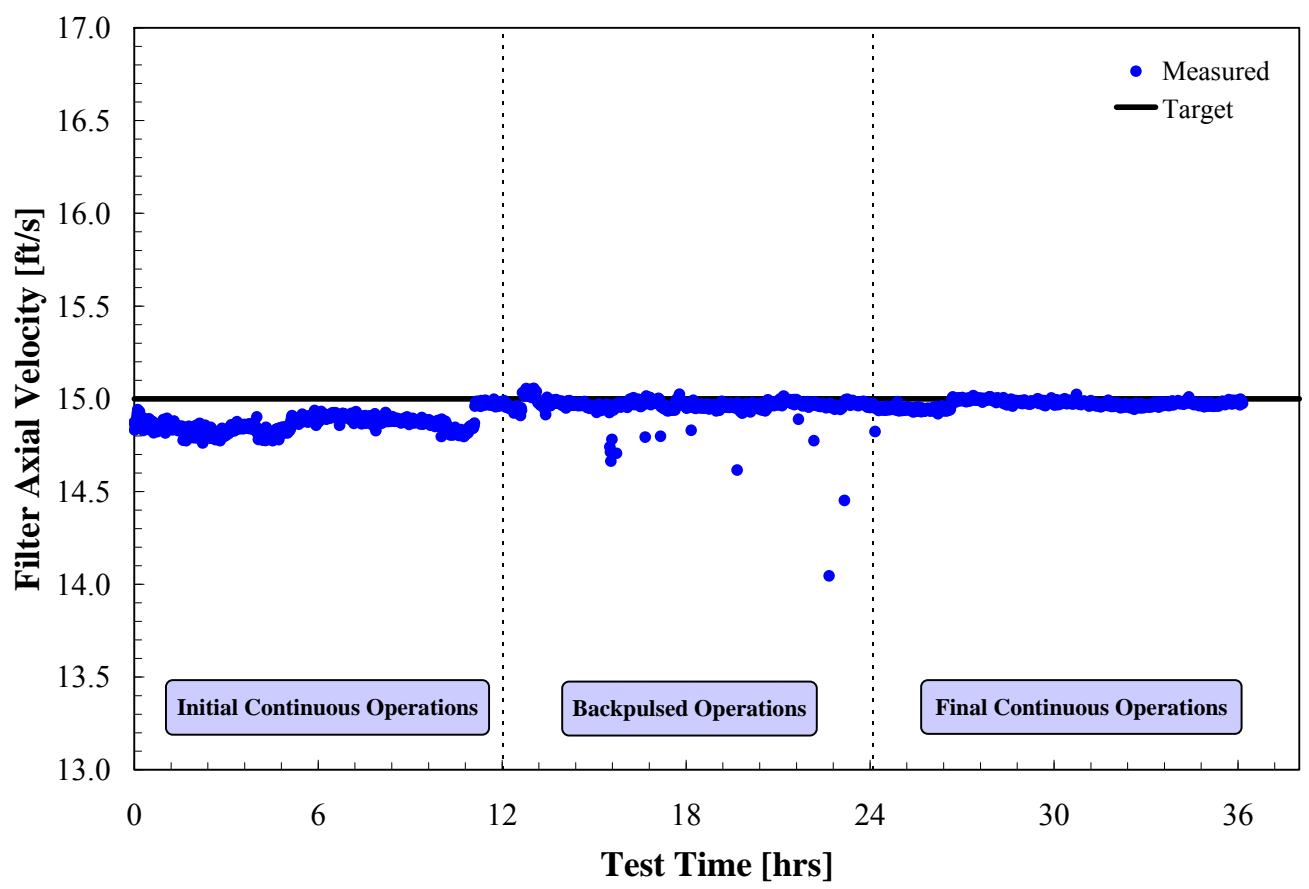

Figure 5.7. CUF Filter AV During Low-Solids Scaling Test \#1. The target AV was $15 \mathrm{ft} / \mathrm{s}$. An average velocity of $14.9 \pm 0.7 \mathrm{ft} / \mathrm{s}(\mu \pm 2 \sigma)$ was achieved.

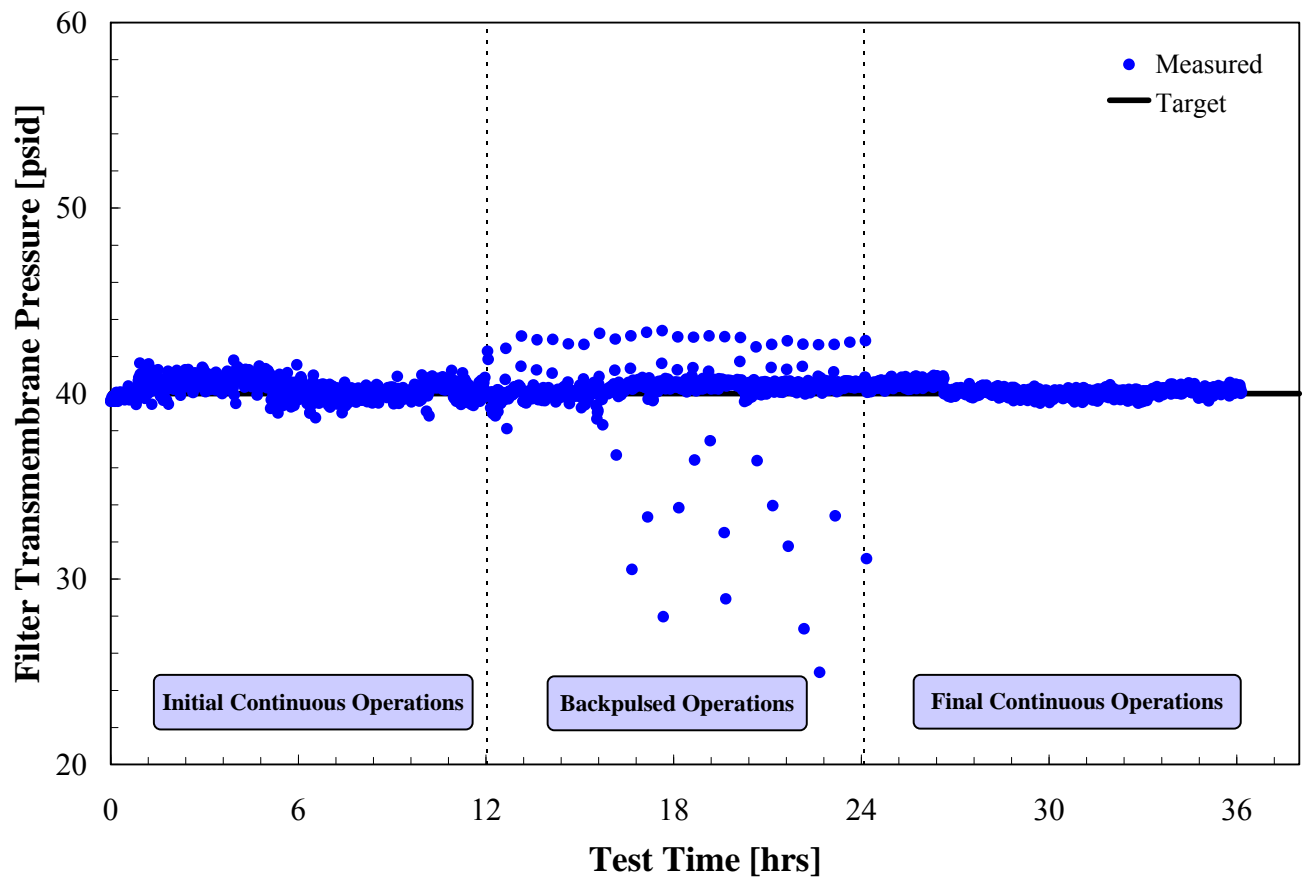

Figure 5.8. CUF Filter TMP During Low-Solids Scaling Test \#1. The target TMP was 40 psid. An average TMP of $40.2 \pm 0.8$ psid $(\mu \pm 2 \sigma)$ was achieved during continuous operations. 


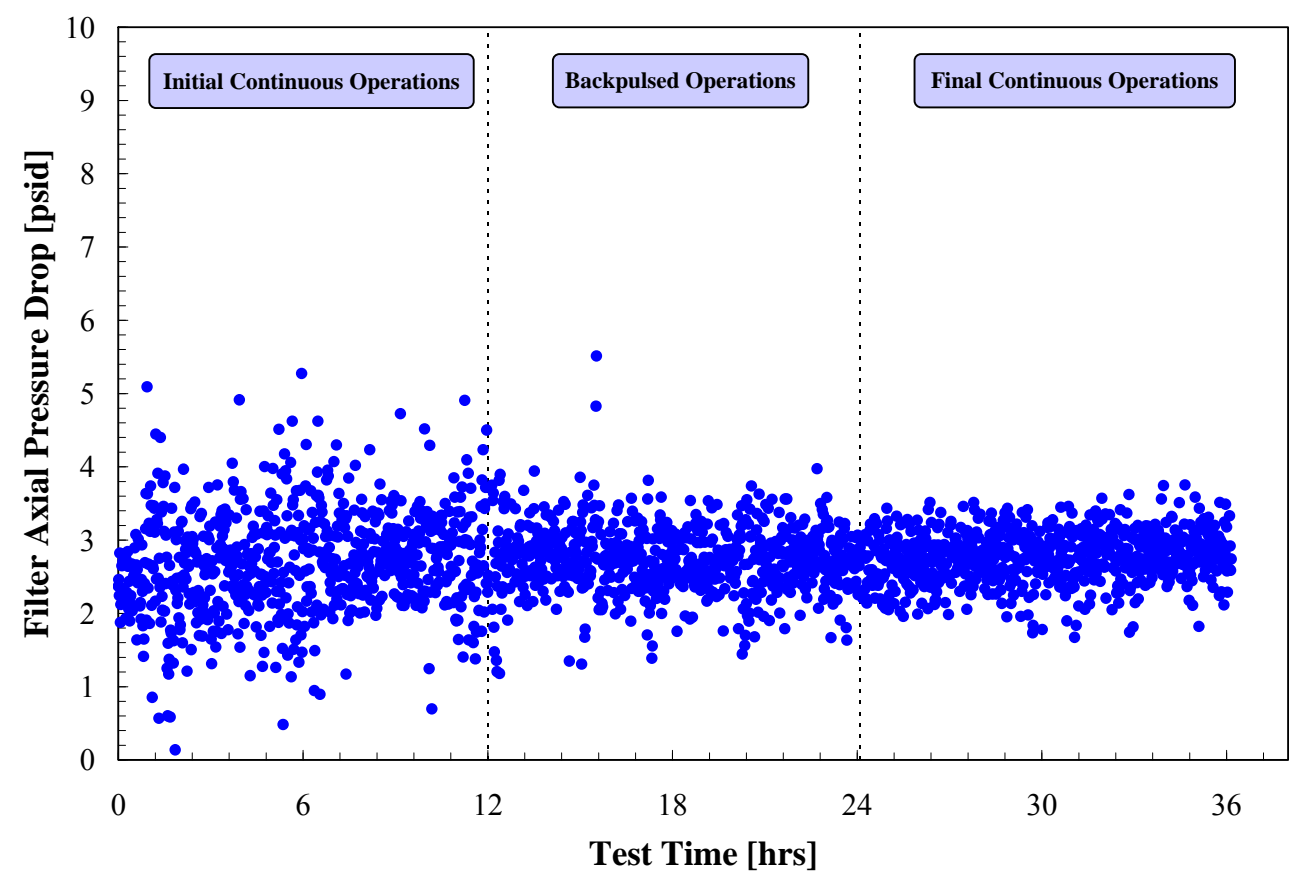

Figure 5.9. CUF Filter APD During Low-Solids Scaling Test \#1

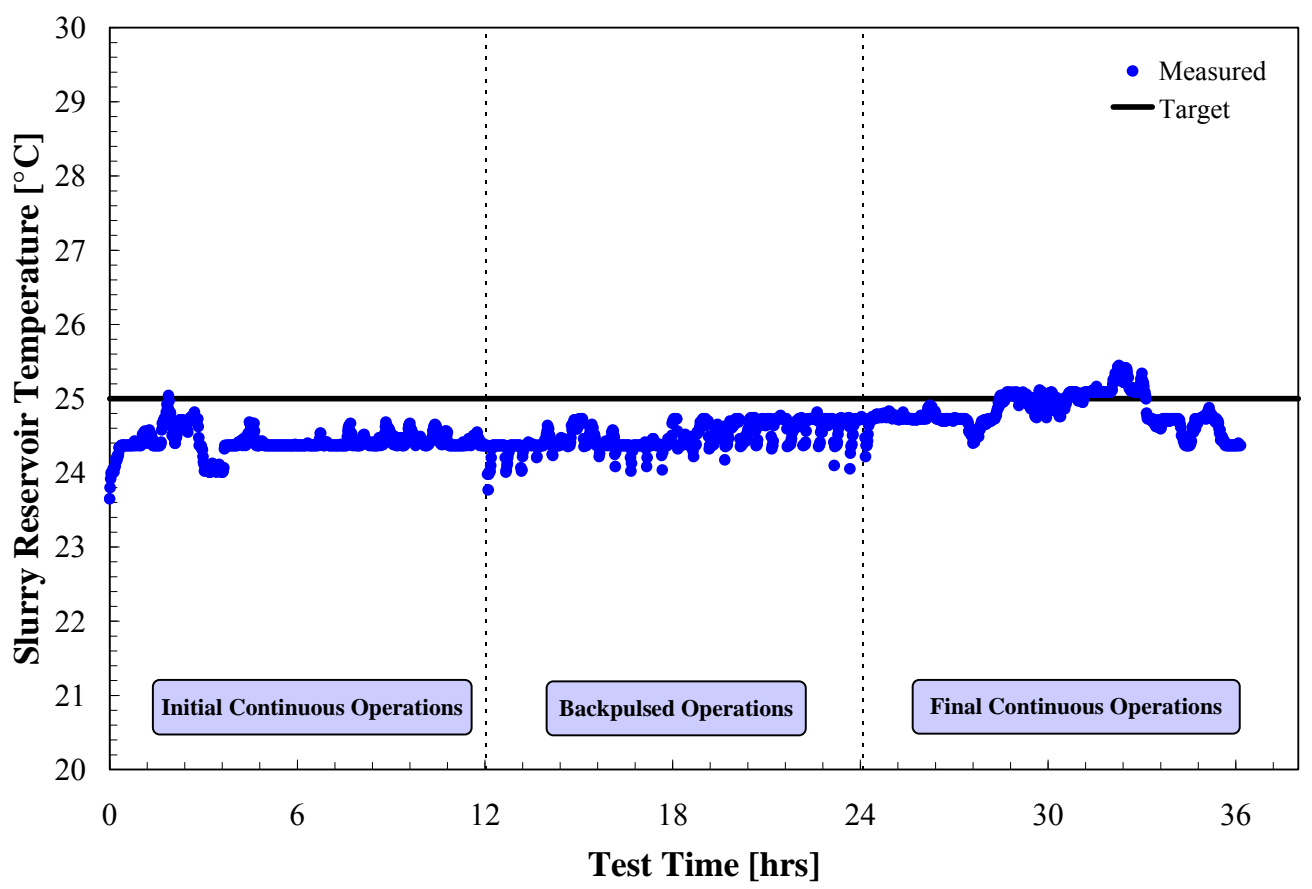

Figure 5.10. CUF Slurry Reservoir Temperature During Low-Solids Scaling Test \#1. The target temperature was $25^{\circ} \mathrm{C}$. An average temperature of $24.6 \pm 0.5^{\circ} \mathrm{C}(\mu \pm 2 \sigma)$ was achieved. 
Figure 5.11 shows the CUF filter flux during Low-Solids Scaling Test \#1 operations. The filter flux decreases from initial values of $0.060 \mathrm{GPM} / \mathrm{ft}^{2}$ down to $0.025 \mathrm{GPM} / \mathrm{ft}^{2}$ by the end of the test. As discussed in previous sections, the transient behavior of filter flux is consistent with filter resistance controlled by cake formation and depth fouling of the porous element.

During the first 12-hr filtration segment, flux declined from $0.060 \mathrm{GPM} / \mathrm{ft}^{2}$ to $0.030 \mathrm{GPM} / \mathrm{ft}^{2}$. This decline was relatively smooth and continuous. The initial backpulse of the filter at a test time of $\sim 12$ hours appeared to restore the original filter flux of $0.060 \mathrm{GPM} / \mathrm{ft}^{2}$ (with the caveat that this value represents a 1-minute average, such that the 1-Hz flux data may show higher, short-lived fluxes following backpulsing). However, continued backpulsing appeared to cause (or allow) a gradual reduction in recovered filter flux. This reduction is consistent with irreversible depth/cake fouling of the filter. As a result, the magnitude and variation in filter flux observed during the final 12-hr period of operation is reduced relative to the initial period. For the final period, the flux declined from an initial value of $0.045 \mathrm{GPM} / \mathrm{ft}^{2}$ to $0.025 \mathrm{GPM} / \mathrm{ft}^{2}$.

From this test sequence of limited duration, it appeared that the flux continued to decrease with time and is asymptotically approaching a minimum filter flux for a given set of process conditions. However, like the PEP filtration tests, a steady state flux condition does not appear to be achieved during CUF operations for Low-Solids Scaling Test \#1. Although current data indicate that CUF and PEP converge as filtration time progresses (see Section 5.1.3), additional study is recommended to determine the minimum (or steady-state) flux for the current simulant to evaluate any potential impacts from filter transience in the scaling-factor analysis, which will allow a "true" evaluation of filter scaling at filtration steady state.

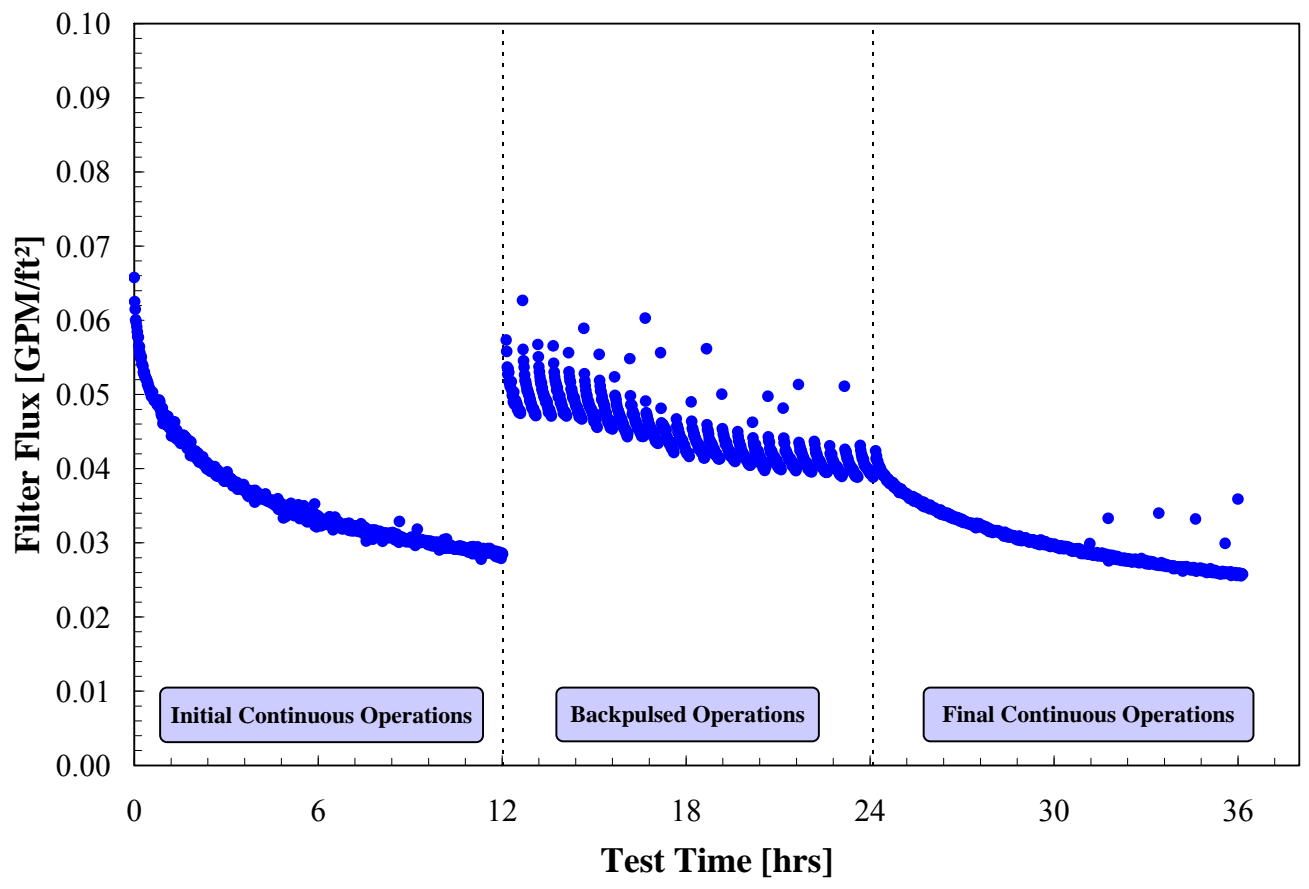

Figure 5.11. CUF Filter Flux (normalized for TMP and temperature variations) During Low-Solids Scaling Test \#1 


\subsubsection{Analysis of Filter Scaling for Low-Solids Test \#1}

To determine the PEP to CUF scaling factor for Low-Solids Test \#1, the relative magnitude of CUF and PEP filter fluxes were compared over the full test duration. This comparison was facilitated by a comparable solids-to-filter surface area ratio of $1.1 \mathrm{~kg} / \mathrm{ft}^{2}$ and $1.5 \mathrm{~kg} / \mathrm{ft}^{2}$ for both PEP and CUF test scales, respectively (see Section 3.4.8). It should be noted that PEP and CUF filters have significantly different test histories. The PEP filter bundles were used only for limited water functional testing before LowSolids Scaling Test \#1. In contrast, the CUF filters were used for a significant number of tests throughout Calendar Year 2008 (see discussion Section 4.1). It is hoped that cleaning of CUF and PEP filters with acid solution should eliminate any difference caused by filter history. However, some of the differences in filter behavior may be attributable to differences in the state of initial filter cleanliness. Because of the complexities of filter cake formation, fouling, and scaling, filter cleanliness effects cannot be separated from scaling effects in the current analysis.

Figure 5.12 shows a comparison of PEP individual filter flux to that measured on the cold-CUF test system. During the first 12-hr segment, the CUF flux was substantially below that observed on all PEP filters. After repeated backpulsing of the filtration systems, the "conditioned" CUF filter flux fell between that of upstream and downstream PEP filter bundles, but did not give an exact measure of either extreme. As such, the results in Figure 5.12 indicate that for the first low-solids scaling test, 1) a comparison of unconditioned filter fluxes (i.e., fluxes measured on filters not appreciably exposed to the slurry solids being treated) at different test scales does not capture filter flux with high accuracy and 2) the CUF provides an inexact indication of individual PEP filter flux. Despite these limitations, CUF does appear to provide an order of magnitude estimate of individual PEP filter bundle filter performance with regard to flux magnitude and filtration dynamics.

Figure 5.13 compares the total PEP flux to the CUF flux. During the first 12 hrs, the CUF filter flux was substantially lower than the PEP flux. Repeated backpulsing of the filter yielded a decline in filter flux for both systems; however, PEP filter flux appears to be more severely impacted. At the end of backpulsing operations, PEP and CUF filter fluxes are similar. As such, both PEP and cold-CUF filtration systems appear to show similar filter fluxes when fully conditioned against the same slurry (as per the final 12-hr segment in Figure 5.13). Relative to individual PEP filter performance, the CUF appears to provide a more accurate representation of total PEP filter performance. While there is a difference between total CUF and PEP flux before filter conditioning, the two fluxes appear to convergence during backpulsed operations and are well matched during the final 12-hr period of operations. 


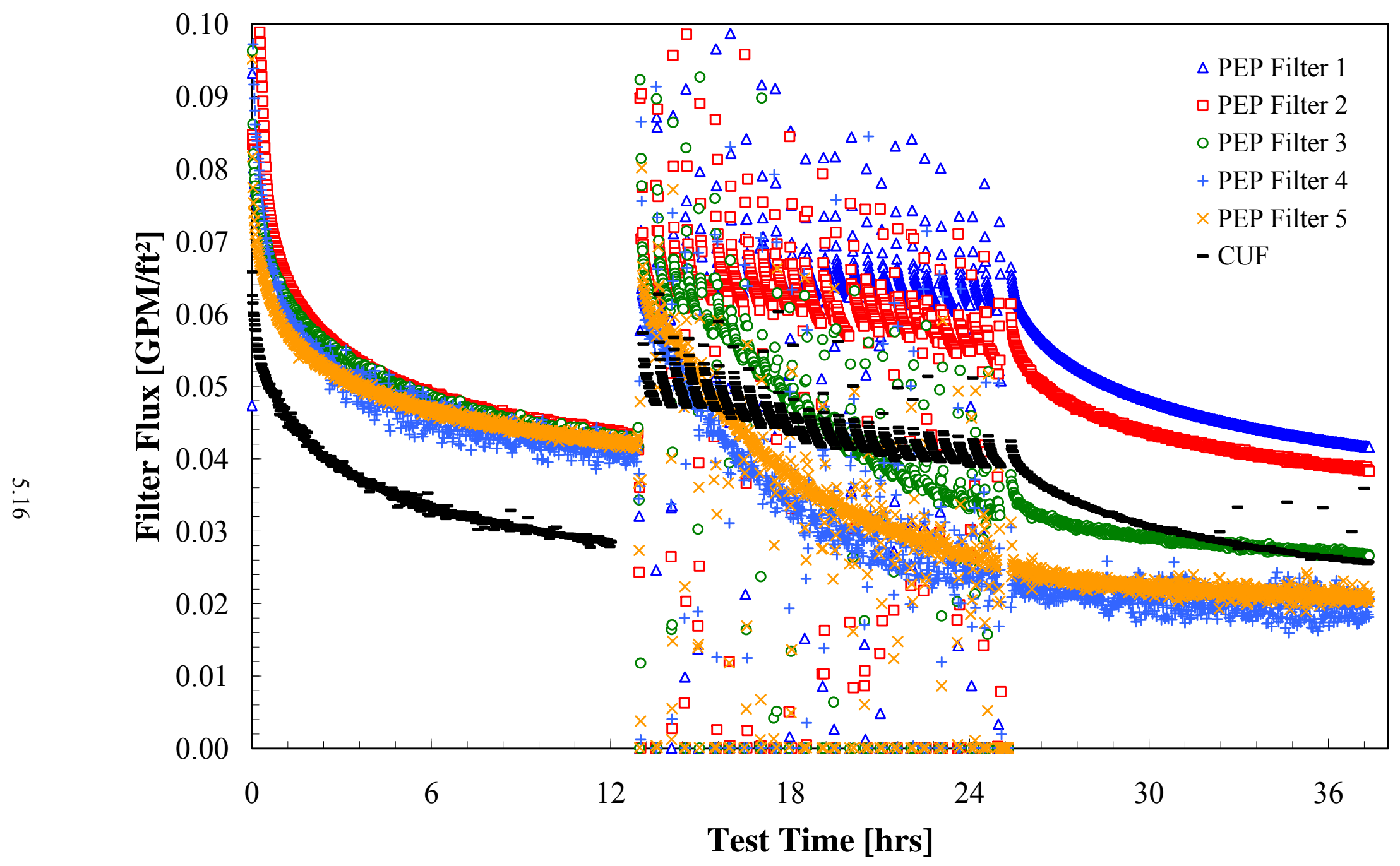

Figure 5.12. Comparison of CUF Filter Flux to Individual PEP Filter Bundle Flux During Low-Solids Scaling Test \#1. All fluxes have been corrected for TMP and temperature variations. The CUF test time scale has been modified to better align the periods of continuous and backpulsed filtration for CUF and PEP. 


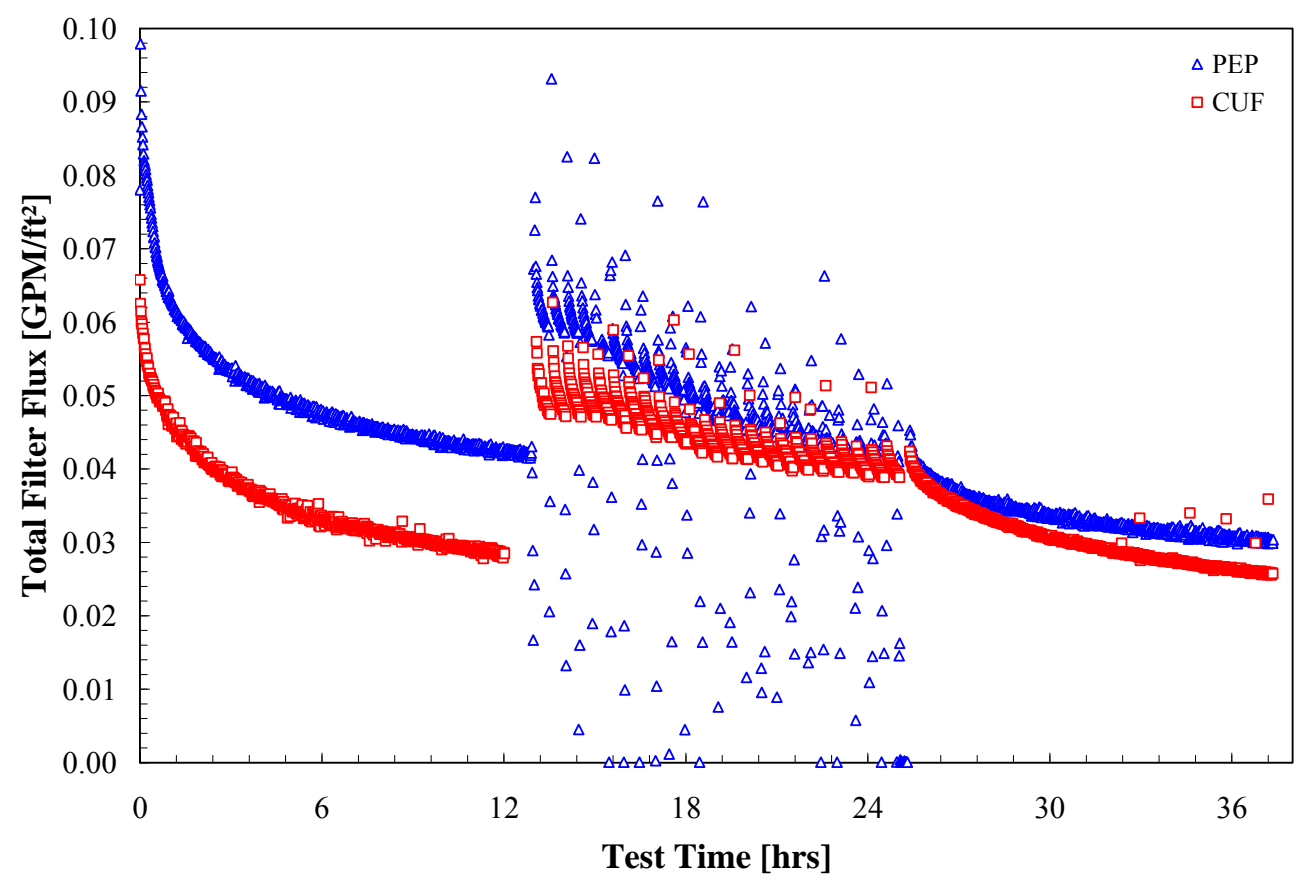

Figure 5.13. Comparison of CUF Filter Flux to Total PEP Filter Flux During Low-Solids Scaling Test \#1. Both fluxes have been corrected for TMP and temperature variations. The CUF test time scale has been modified to better align the periods of continuous and backpulsed filtration for CUF and PEP.

The CUF and PEP filter flux tests can be used to assess filter scaling from the bench test system to the engineering test system. Figure 5.14 and Figure 5.15 show the results of the scaling factor analysis. It should be noted that the scaling analysis accounts for differences in the test times between the scaling tests. The start of continuous and backpulsed operations for all tests has been aligned for CUF and PEP testing. Initial continuous operations were time shifted so that the start of filtration CUF and PEP matched and such that initial continuous filtration data beyond the planned $12 \mathrm{hrs}$ of operation were discounted. The flux data in the initial continuous region was averaged over 30-minute intervals to create 24 individual flux measurements (over a 12-hr nominal test period) for comparison. Backpulsed operations were aligned by matching backpulses. For both CUF and PEP, the recovered flux between backpulses was time averaged to create two sets of 24 flux measurements for scaling analysis. Finally, fluxes for the final continuous operations were matched using the filter backpulse at the start of this final 12-hr period. Like before, the flux was averaged over 30-minute intervals to create 24 separate averaged flux measurements for PEP to CUF scaling analysis. The time corrections outlined above yield a total of 72 flux measurements spanning a 36-hour period (hereafter referred to as the "nominal test time").

Examined as scaling factors for individual filters (Figure 5.14), the scaling factor $(S)$ ranges from 1.2 to 1.6 for the first $12 \mathrm{hrs}$ of operations (where the filters are relatively "unconditioned"). That the scaling factor is greater than 1 indicates that the CUF flux under predicts that for PEP. During the period of backpulsed operations, scaling factors diverge from an initial value of $\sim 1.2$ to values that range from 0.6 to 1.6. This difference reflects the significant decline in the down-stream filters at PEP and the absence of 
a similarly strong decline in CUF. This spread in individual scaling factors persisted into and throughout the final 12-hr period of operation.

When examined on a total filter flux basis (as in Figure 5.15), the scaling factor ranges from $\sim 1.3$ to 1.5 in the first 12-hr segment (unconditioned filters), 1.2 to $\sim 0.9$ during backpulse operations, and approximately 1.0 to 1.2 in the final $12-\mathrm{hr}$ segment (conditioned filters). For the majority of Low-Solids Scaling Test \#1, the cold-CUF test system under predicts the PEP scale flux, yielding scaling factors greater than one. The degree of under prediction is significant at the start of testing when the filters are not fully conditioned. During backpulsing and at the end of testing (i.e., when the filters are strongly conditioned), the total scaling factor appears to be close to or slightly greater than one.

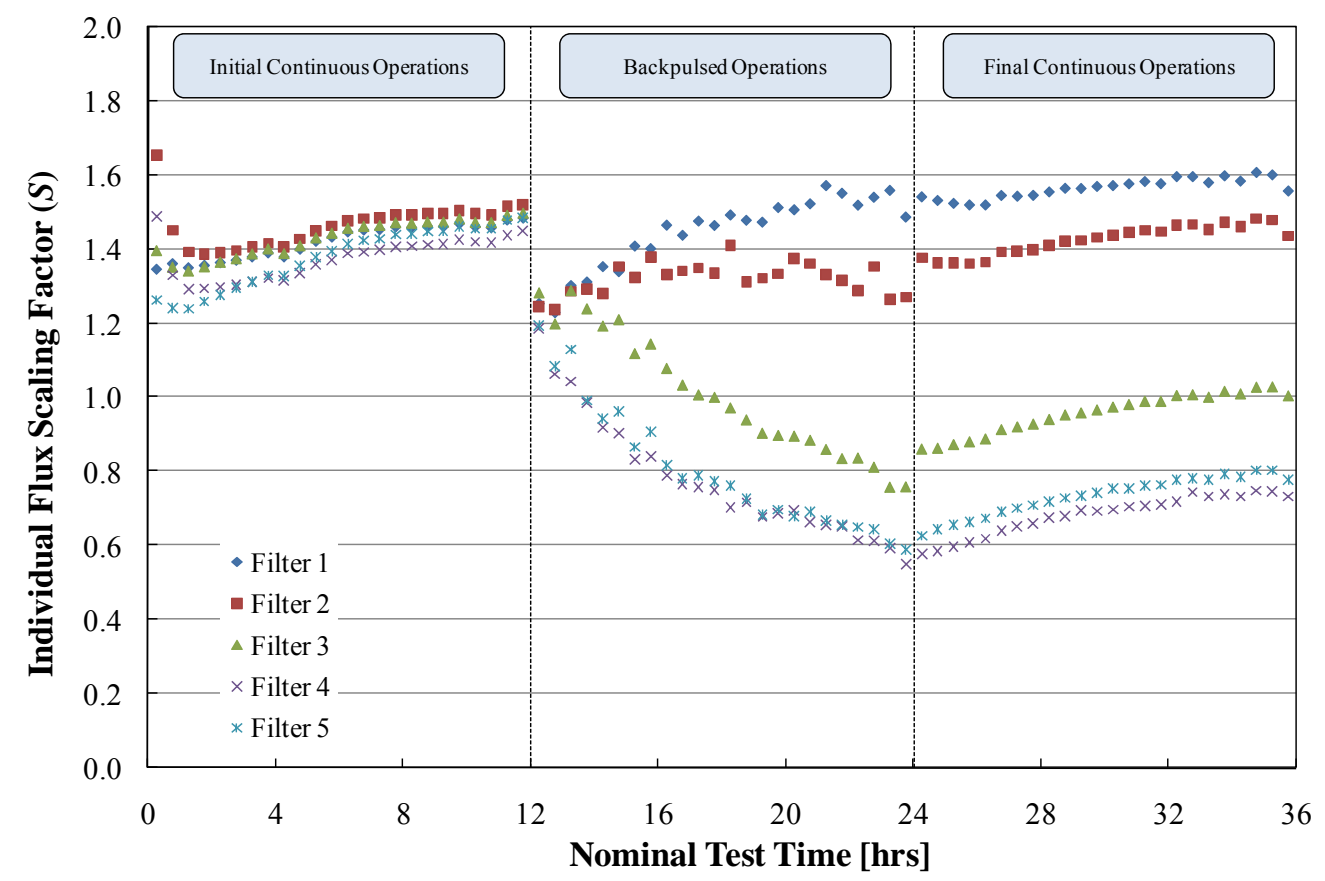

Figure 5.14. PEP to CUF Scaling Factor all Five PEP Filters as a Function of Time for Low-Solids Scaling Test \#1. Each scaling factor value has an associated error of approximately $10 \%$. 


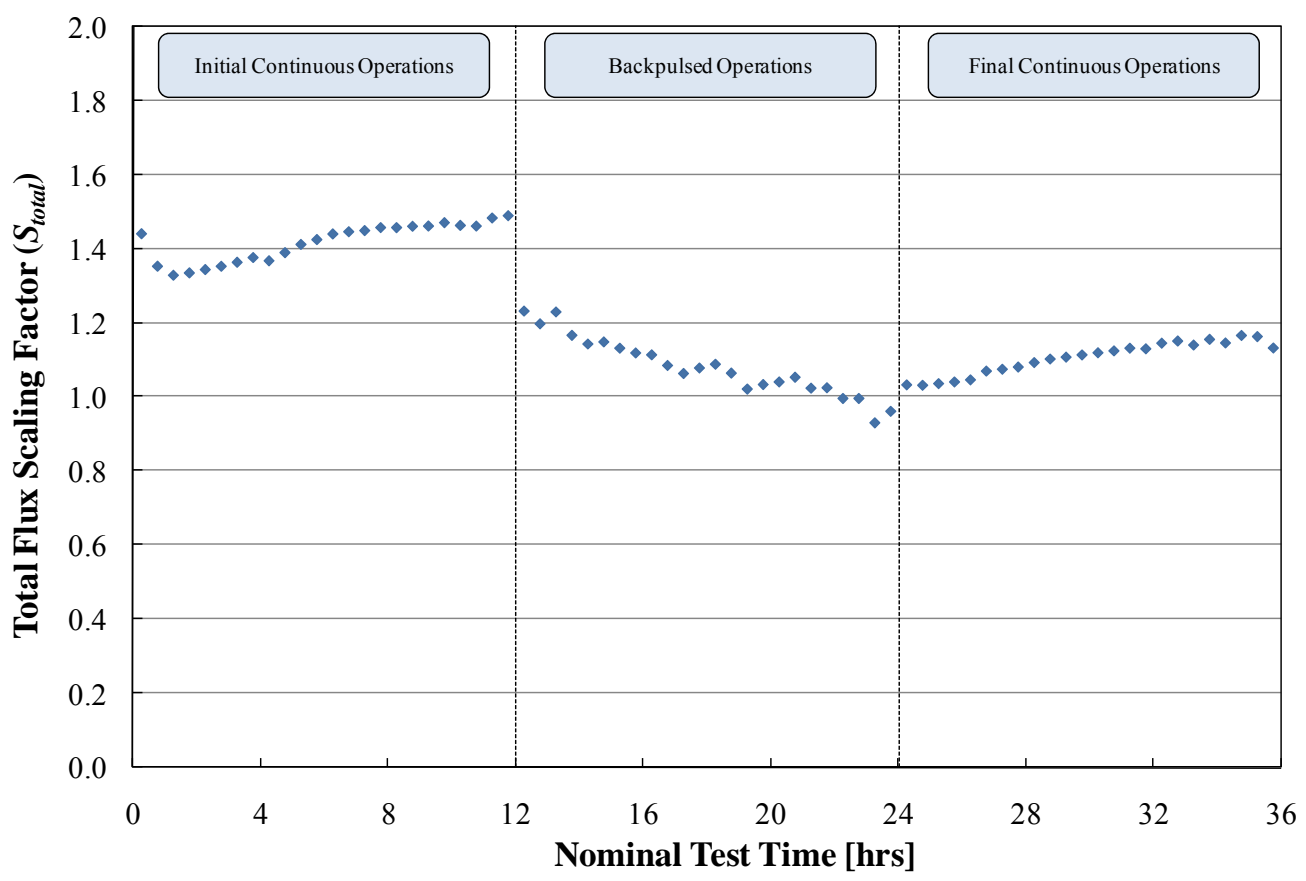

Figure 5.15. Total PEP to CUF Scaling Factor as a Function of Time for Low-Solids Scaling Test \#1. Each scaling factor value has an associated error of approximately $10 \%$.

As stated in preceding paragraphs, the results of Low-Solids Scaling Test \#1 indicate that total CUF flux provides only an approximate measure of filtration performance of individual PEP elements (in terms of flux magnitude and time-dependency). For the current analysis, the consequence of this appears to be a highly variable scaling factor ( $\sim 0.7$ to 1.6 at the end of testing) when considered on an individual filter basis.

Table 5.4 provides a summary of scaling factors (as individual and total scaling factors) for the initial continuous 12-hr period, the backpulsed 12-hr period, and the final continuous $12-\mathrm{hr}$ period of operations. The results shown in Table 5.4 are simply averages of scaling factors shown in Figure 5.14 and Figure 5.15 over the given period of operation. Of the results shown, the most appropriate results for scaling analyses appear to correspond to the total scaling factors for backpulsed and final continuous operations $(1.1 \pm 0.1$ for both). These scaling factors are appropriate for systems with significant exposure of the filter to waste solids. In terms of the current test, it is conservative in that it takes into account the observed flux losses from flux divergence during backpulsed operations. The uncertainty associated with these results represents two standard deviations and is derived from estimates of fluctuations in the permeate collection rates and permeate density that have been propagated through the scaling factor analysis equations outlined in Section 3.4.8. For the current analysis, these uncertainties suggest that the PEP to CUF scaling factor is near or slightly larger than one. To provide a conservative estimate for process scaling, a scaling factor of 1.0 is recommended (based on the current test results). Depending on how the scaled operations are operated, scaling factors corresponding to the initial continuous period of operations may be more appropriate. In this case, use of a scaling factor of 1.0 would also appear to provide a conservatively low estimate of scaled filter flux based on the test results shown in Table 5.4. 
Table 5.4. Average PEP to CUF Scaling Factors for Filter Flux as a Function of Filter Bundle and as a Function of Process Operation. Scaling factors were derived from Low-Solids Scaling Test \#1. Reported uncertainties represent two sample standard deviations $(\mu \pm 2 \sigma)$.

\begin{tabular}{lcccccc}
\hline & \multicolumn{5}{c}{ Average Scaling Factor $\left(S_{i}\right)$} \\
\cline { 2 - 7 } Process Operation & Filter 1 & Filter 2 & Filter 3 & Filter 5 & Filter 6 & Total \\
\hline Initial Continuous & $1.4 \pm 0.2$ & $1.5 \pm 0.2$ & $1.4 \pm 0.2$ & $1.4 \pm 0.1$ & $1.4 \pm 0.1$ & $1.4 \pm 0.2$ \\
Backpulsed & $1.4 \pm 0.2$ & $1.3 \pm 0.1$ & $1.0 \pm 0.1$ & $0.8 \pm 0.1$ & $0.8 \pm 0.1$ & $1.1 \pm 0.1$ \\
Final Continuous & $1.6 \pm 0.2$ & $1.4 \pm 0.2$ & $1.0 \pm 0.1$ & $0.7 \pm 0.1$ & $0.7 \pm 0.1$ & $1.1 \pm 0.1$ \\
\hline
\end{tabular}

\subsection{Test Results for Low-Solids Scaling Test \#2}

PEP operations for Low-Solids Scaling Test \#2 were conducted from $2314 \mathrm{hrs}$ December $30^{\text {th }}, 2008$, to 1127 hrs January $1^{\text {st }}, 2009$, PST. PEP process data were recorded by the PEP DAS at a frequency of 1 Hz. For subsequent analyses, all relevant $1-\mathrm{Hz}$ data (i.e., those over the process times of interest) were pulled and averaged over 1-minute intervals. The slurry sample for bench-scale tests (ID\# S 02AML 008 XX 0868 CUF 4) was taken at 2331 hrs December $30^{\text {th }}, 2008$, from the middle-low sampling port in Tank T02A. This sample was delivered to APEL for cold-CUF testing. The sampling time occurred after the start of testing, and as such, CUF and PEP test slurries should be equivalent in slurry composition and UDS concentration. Parallel CUF operations were conducted from $0748 \mathrm{hrs}$ January $5^{\text {th }}, 2009$, to $2001 \mathrm{hrs}$ January $6^{\text {th }}, 2009$, PST. CUF data were recorded by the CUF DACS at a frequency of $0.4 \mathrm{~Hz}$. The CUF DACS then averages all $0.4 \mathrm{~Hz}$ data over 1-minute process intervals. For all CUF data analyses, the 1-minute data averages were employed. The test operations and scaling results for LowSolids Test \#2 are discussed in detail in the following sections.

\subsubsection{PEP Operations}

PEP operations for Low-Solids Scaling Test \#2 employed a circulating slurry mass of approximately $1500 \mathrm{~kg}$ of a $6.9-\mathrm{wt} \%$ UDS concentration waste simulant slurry (based on estimates and analysis of tank levels and transfer volumes). This slurry mass yields a slurry solids-to-filter surface area ratio of $1.4 \mathrm{~kg} / \mathrm{ft}^{2}$. As stated before, this ratio is lower than anticipated for use in WTP (i.e., 1.7 to $16 \mathrm{~kg} / \mathrm{ft}^{2}$ —see Section 5.1.1) and is also higher than employed for the first PEP low-solids scaling test $\left(1.1 \mathrm{~kg} / \mathrm{ft}^{2}\right)$. Previous scaling studies (Daniel et al. 2009) suggest that this difference is not expected to impact filtration scaling substantially (see Section 3.4.8). As such, this low-solids scaling test is expected to be representative of the lower bound of PTF operations with respect to the slurry solids-to-filter surface area ratio.

Table 5.5 provides a summary of physical properties for the slurry tested in Low-Solids Scaling Test \#2. PEP process measurements, including operational parameters and filter flux results, are shown in Figure 5.16 to Figure 5.21. The filter AVs, as measured by flow sensors at the suction and discharge of the filtration loop pumping system, is shown in Figure 5.16. During the periods of continuous nonbackpulsed operations, AV appeared to gradually decrease. To correct this, pumping rate adjustments were made periodically through the test and appear as discontinuities in the AV rates. During backpulsing operations, AV shows little to no transience but is subject to significant scatter as a result of backpulsing. Overall, average velocities of $15.1 \mathrm{ft} / \mathrm{s}$ and $14.8 \mathrm{ft} / \mathrm{s}$ (at the suction and discharge to the pumps, respectively) were achieved against a test target of $15.0 \pm 1.4 \mathrm{ft} / \mathrm{s}$. 
Figure 5.17 shows the TMP achieved for PEP filter bundles 1 to 5 during Low-Solids Scaling Test \#2. TMPs were stable throughout testing; scatter during backpulsed operations results from including back pressures in the TMP averages. Average TMPs for the test fell within \pm 0.5 psid of the target TMP of 40 psid and met the allowable variation of \pm 4 psid. The actual TMP measurements shown in Figure 5.17 were used to correct filter flux for deviations from the target TMP. Table 5.6 shows the operational parameters for Tank T02A PJM sparging systems that were achieved in Low-Solids Scaling Test \#2. Table 5.6 indicates that most target conditions were met (within acceptable tolerances) with exception of the Tank T02A PJM jet velocity. The PJM velocity is comparable to that for Low-Solids Scaling Test \#1.

Table 5.5. PEP Slurry Properties for Low-Solids Scaling Test \#2

\begin{tabular}{lcl}
\hline Property & Measured Value & Units \\
\hline Approximate Slurry Mass Tested & 1500 & $\mathrm{~kg}$ \\
Slurry Solids-to-Filter Area Ratio & 1.4 & $\mathrm{~kg} / \mathrm{ft}^{2}$ \\
Rheology & & \\
$\quad$ Bingham Yield Stress & $0.06 \pm 0.01$ & $\mathrm{~Pa}$ \\
$\quad$ Bingham Consistency & $4.9 \pm 0.1$ & $\mathrm{mPa} \cdot \mathrm{s}$ \\
$\quad$ Supernate Viscosity & 2.8 & $\mathrm{mPa} \cdot \mathrm{s}$ \\
Density & $1.29 \pm 0.01$ & $\mathrm{~kg} / \mathrm{L}$ \\
$\quad$ Slurry Bulk Density & $1.23 \pm 0.03$ & $\mathrm{~kg} / \mathrm{L}$ \\
$\quad$ Permeate Density & & \\
Solids Concentrations & $32.1 \pm 0.2$ & $\mathrm{wt} \%$ \\
$\quad$ Total Solids & $6.93 \pm 0.04$ & $\mathrm{wt} \%$ \\
$\quad$ Undissolved Solids & $27.1 \pm 0.3$ & $\mathrm{wt} \%$ \\
$\quad$ Dissolved Solids In Permeate & $38.4 \pm 1.2$ & $\mathrm{wt} \%$ \\
$\quad$ Centrifuged Solids & &
\end{tabular}

Table 5.6. Operation Conditions Achieved for Tank T02A PJM and Sparging Systems During LowSolids-Scaling Test \#2

\begin{tabular}{lcc}
\hline Parameter & Target & Actual \\
\hline Tank T02A PJM Jet Velocity & $7.3 \pm 0.4 \mathrm{~m} / \mathrm{s}$ & $6.3 \mathrm{~m} / \mathrm{s}$ \\
Tank T02A PJM Cycle Time & $33 \pm 1 \mathrm{~s}$ & $33.2 \mathrm{~s}$ \\
Tank T02A PJM Stroke Length & $30.3 \pm 1.8 \mathrm{in}$. & $29.2 \mathrm{in.}(77 \%)$ \\
Tank T02A Steam Ring Purge Flowrate & $0.10 \pm 0.02 \mathrm{~kg} / \mathrm{min}$ & $0.10 \mathrm{~kg} / \mathrm{min}$ \\
Tank T02A Upper Air Sparger Flowrate & $0.10 \pm 0.02 \mathrm{~kg} / \mathrm{min}$ & $0.10 \mathrm{~kg} / \mathrm{min}$ \\
Tank T02A Total Lower Air Sparger Flowrate & $0.40 \pm 0.05 \mathrm{~kg} / \mathrm{min}$ & $0.39 \mathrm{~kg} / \mathrm{min}$ \\
Number of Filter-Loop Bundles & 5 & 5 \\
\hline
\end{tabular}




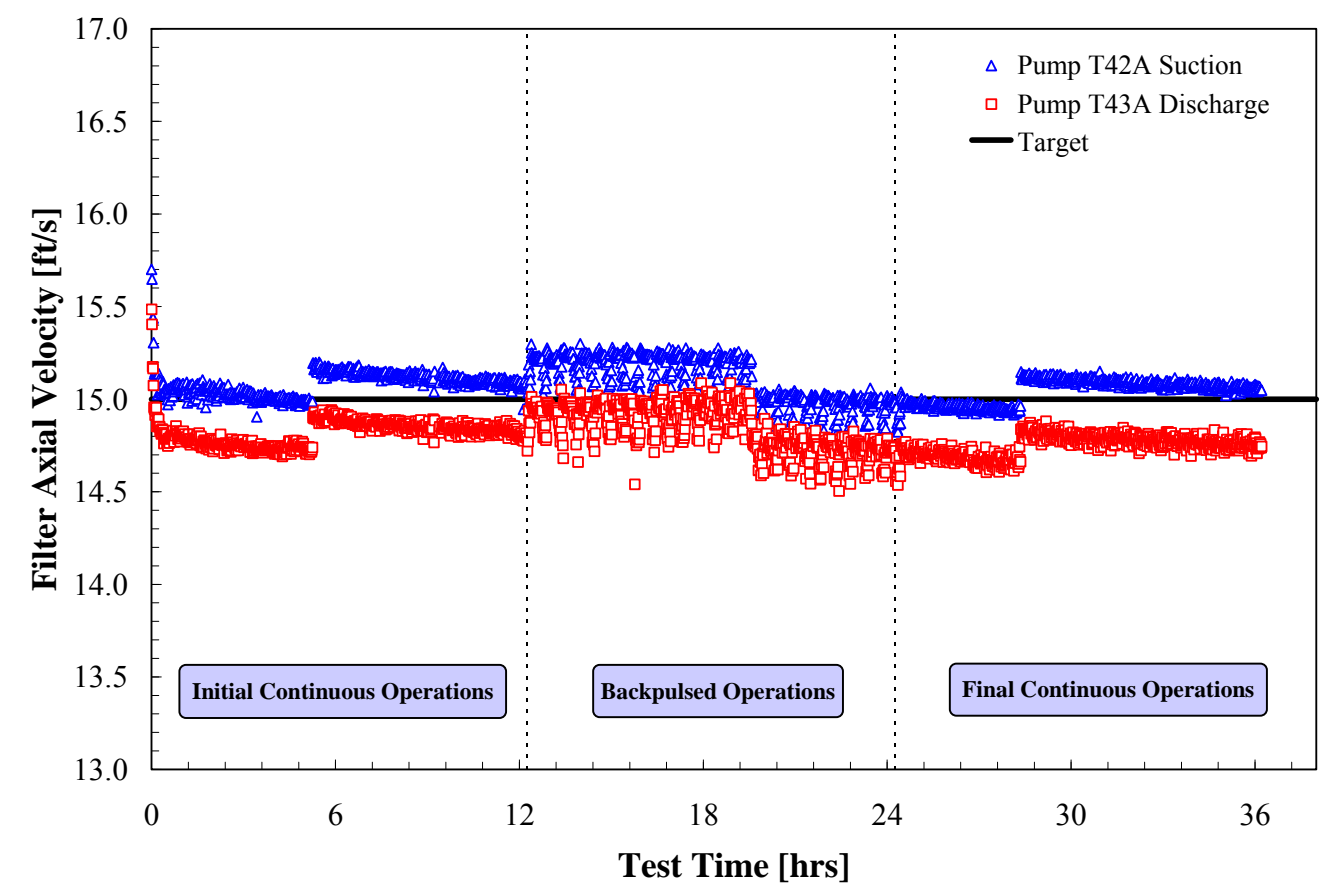

Figure 5.16. Filter AVs Achieved at PEP During Low-Solids Scaling Test \#2. The velocities at Pump T42A suction and Pump T43A discharge are based on sensors FT-0623 and FT-0635, respectively. The target velocity was $15.0 \pm 1.4 \mathrm{ft} / \mathrm{s}$. Average velocities of $15.1 \mathrm{ft} / \mathrm{s}$ and 14.8 (at the suction and discharge to the pumps, respectively) were achieved.

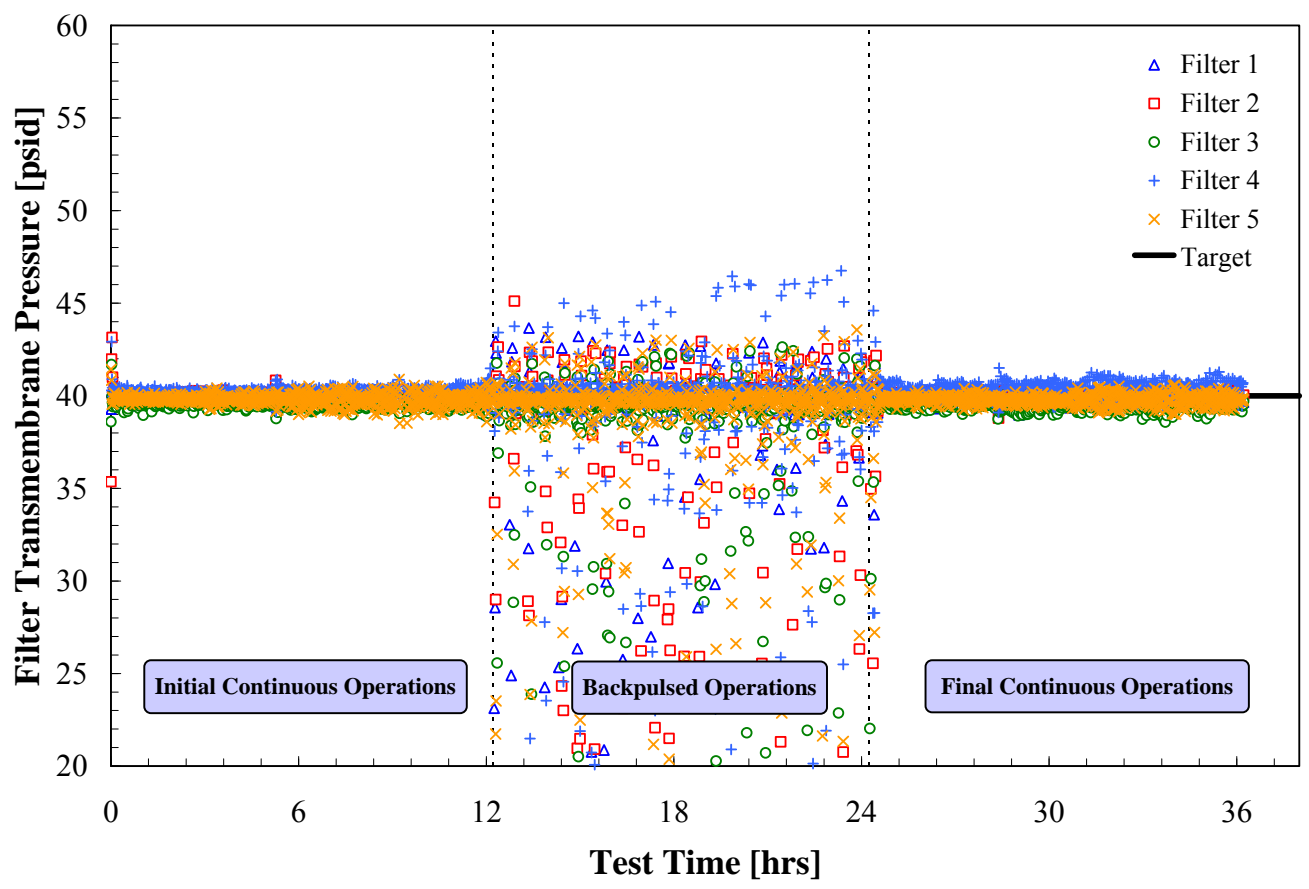

Figure 5.17. Filter TMPs Achieved at PEP During Low-Solids Scaling Test \#2. The target TMP for all filters was $40 \pm 4$ psid. The average TMP for all filters fell within \pm 0.5 psid of 40 psid. 
Figure 5.18 shows the APDs across each of the PEP filter bundles during Low-Solids Scaling Test \#2. All APDs fell below the upper limit of 25 psid for the test. As expected, the APD across bundles containing 10-ft filter elements (filters 1 to 3) was higher than that of the bundles containing the 8-ft filter elements (filters 4 and 5). Figure 5.19 shows the prototypic tank temperature in vessel T02A during LowSolids Scaling Test \#2. This temperature curve is used to correct permeate flux for deviations in test temperature from the target of $25 \pm 2{ }^{\circ} \mathrm{C}$. The average temperature for Tank T02A during testing was $25.3^{\circ} \mathrm{C}$. It should be noted that the temperature of the filtration loop was likely a few degrees higher as a result of mechanical heat input from pumps T42A and T43A. This difference introduces uncertainty between CUF and PEP temperature corrections to flux because of different heat exchange configurations in the CUF and PEP test systems (see Section 4.4).

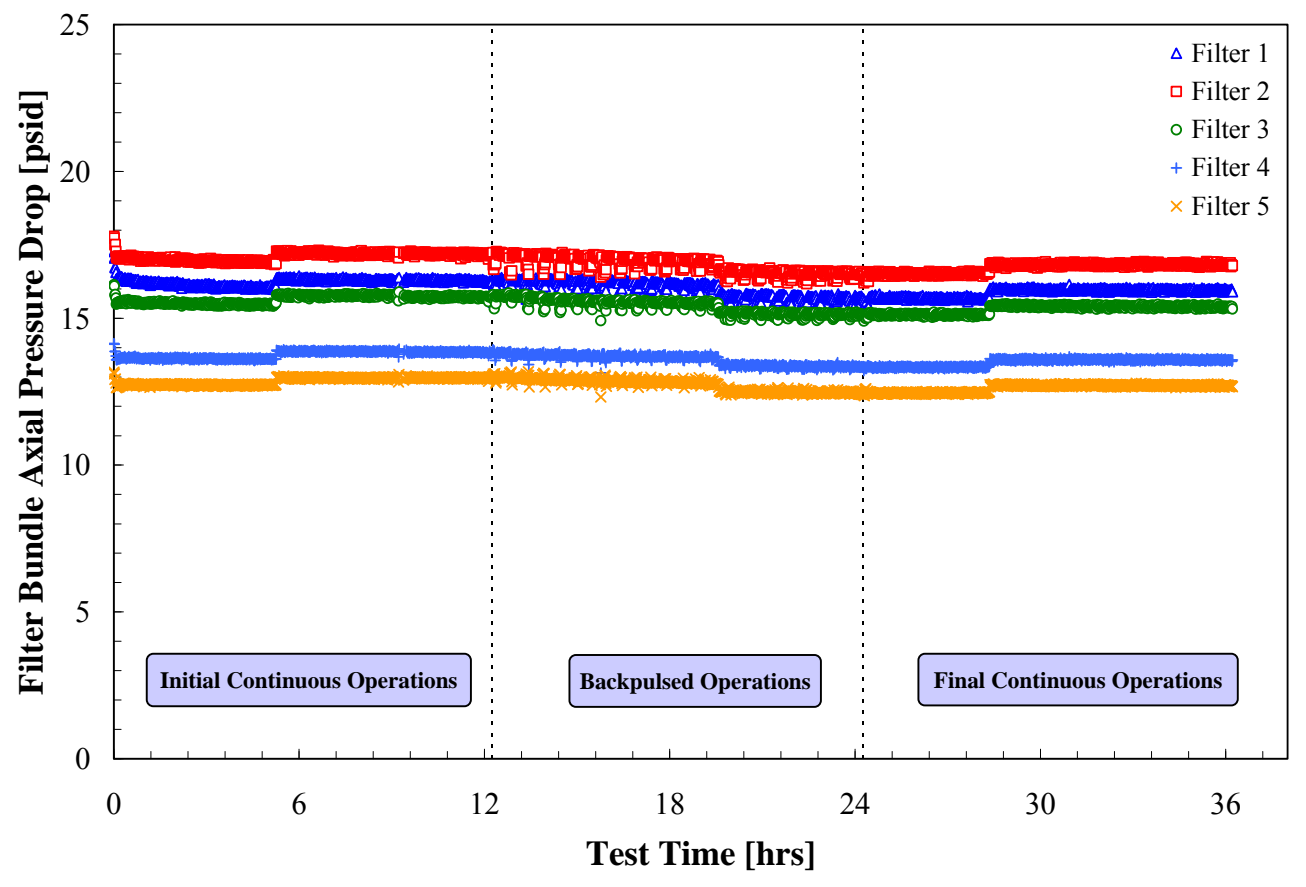

Figure 5.18. Filter APDs Observed at PEP During Low-Solids Scaling Test \#2. The upper allowable limit for APD was 25 psid for all filters. 


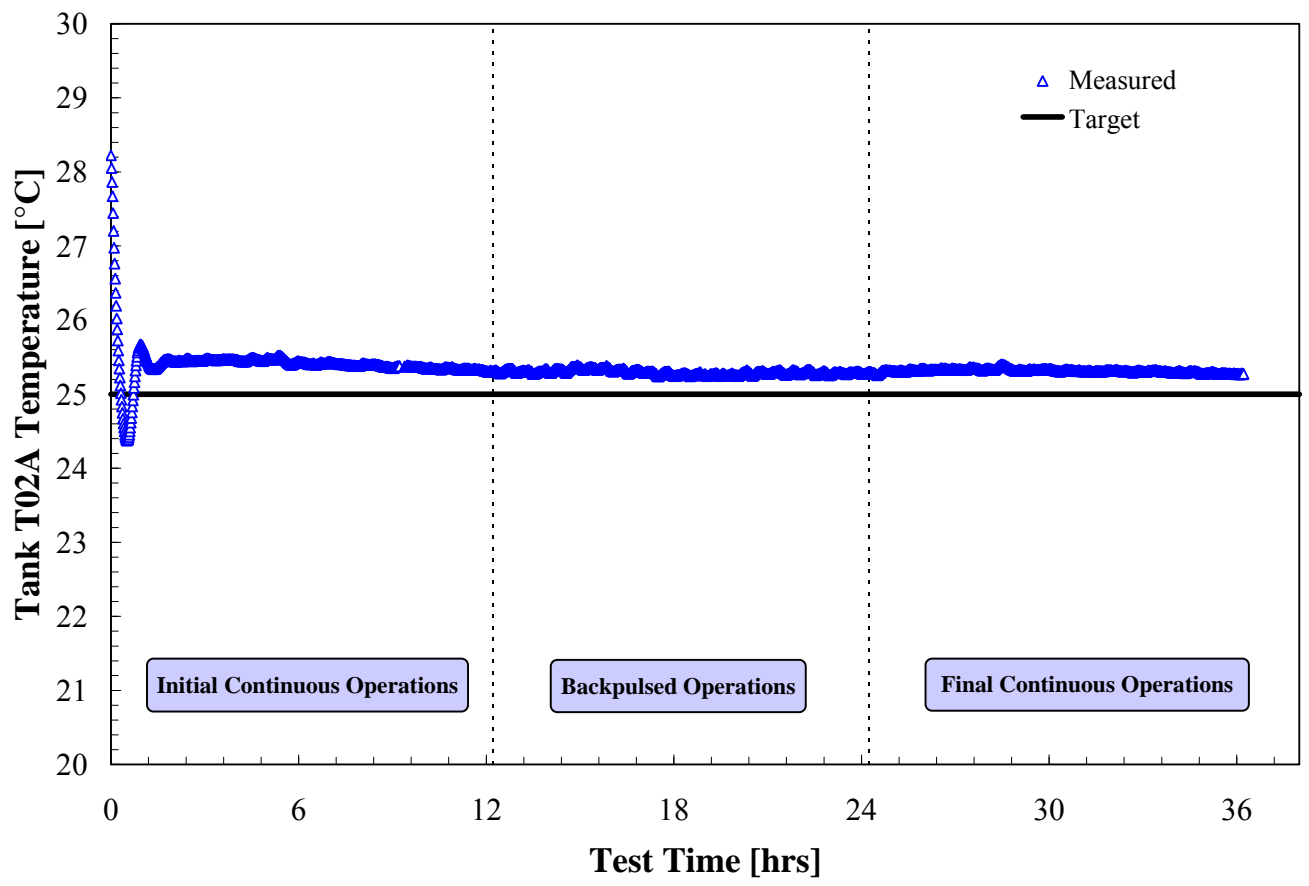

Figure 5.19. Temperature of Vessel UFP-VSL-T02A During Low-Solids Scaling Test \#2. The target temperature of the filtration loop during testing was $25 \pm 2^{\circ} \mathrm{C}$. The average temperature for Tank T02A was $25.3^{\circ} \mathrm{C}$.

Figure 5.20 shows the temperature and TMP corrected flux for PEP filter bundles 1 to 5 during LowSolids Scaling Test \#2. The filter flux behavior during each of the three test periods (i.e., initial continuous non-backpulsed, backpulsed, and final continuous non-backpulsed operations) generally match those observed in first low-solids scaling test (see Figure 5.5 and associated discussion) with a few exceptions.

During the first 12-hr segment, all filters (with exception of filter 1) show a smooth decline in flux from $0.070 \mathrm{GPM} / \mathrm{ft}^{2}$ to $0.035 \mathrm{GPM} / \mathrm{ft}^{2}$ that is consistent with typical cake and filter depth fouling mechanisms observed in previous filtration operations. It should be noted that for the current test results, the flux for filter 1 starts off much lower (at $0.039 \mathrm{GPM} / \mathrm{ft}^{2}$ ) than that for bundles 2-5 and only declines weakly to a final flux of approximately $0.030 \mathrm{GPM} / \mathrm{ft}^{2}$ at $12 \mathrm{hrs}$. This deviation appears to result from reversible cake fouling, as indicated by recovery of filter bundle 1 flux to a level that matches bundles 2-5 when backpulsed at $12 \mathrm{hrs}$.

As described in Section 5.1.1, the start of filtration for the conditioning test was identified as the permeate rate maximum following the initiation of filtration. This point was selected because the filters were not backpulsed before the start of filtration as was done in previous filter conditioning tests outlined in Russell et al. (2009c). The selected start time for filtration was subject to the constraints that the pulse pots be filled with permeate and that the filtration conditions be achieved. With regard to the latter, the target TMP and AV had to have been reached, and filtration on all five filters had to have been started. The data for Low-Solids Test \#2 indicate the time corresponding to these conditions to be $2314 \mathrm{hrs}$ on December $30^{\text {th }}, 2008$. It should be noted that before this time, permeate was allowed to flow through only filter bundle \#1 for approximately 15 minutes at target TMP and AV (but not through the other filters). 
This brief period of filtration likely caused cake formation on filter bundle 1 while the other filters (which had no permeate flow) remained relatively clean. As such, the brief period of filtration on bundle 1 preceding low-solids scaling test (and the lack of backpulsing at the start of the test) are the likely cause for the low-filter flux observed on filter bundle 1 during the initial 12-hr period of operations in Figure 5.20.

Relative to the initial flux observed during the first low-solids scaling test $\left(\sim 0.1 \mathrm{GPM} / \mathrm{ft}^{2}\right.$ at time zero), the initial flux observed in the second low-solids scaling test is low. Although the exact cause for this difference is unknown, it could be attributed to 1) potential differences in the initial state of filter cleanliness (particularly depth foulants resistant to the filter cleaning regimen) between the two scaling tests or 2) differences in the slurry supernate composition between tests (as indicated by differences in the dissolved solids concentration in Table 5.1 and Table 5.5). With regard to the first point, it highlights the potential importance of filter cleaning and history in determining initial flux. However, it is difficult to separate these issues from potential scaling issues based on the current information available.

The initial backpulse leading into the second 12-hr test segment yielded a recovery of all filter fluxes to approximately $0.055 \mathrm{GPM} / \mathrm{ft}^{2}$ (again with the caveat that this flux represents a 1-minute average and that the actual flux may have been larger for a short period of time). However, repeated backpulsing of the filters throughout the second test segment appears to cause a divergence in the recovered filter flux across the filter array. Specifically, the flux on bundles 1 and 2 remained high and showed only a slight decline to $0.050 \mathrm{GPM} / \mathrm{ft}^{2}$ throughout backpulsed operations. In contrast, the recovered flux for bundles 3 , 4 , and 5 , show a marked decrease down to $0.030 \mathrm{GPM} / \mathrm{ft}^{2}$ by the end of backpulsed operations. The difference in filter flux caused by flux divergence during backpulsed operations persisted into and throughout the final 12-hr test segment. Relative to the downstream filters, bundles 1 and 2 showed a high flux that starts at $0.050 \mathrm{GPM} / \mathrm{ft}^{2}$ and declined to $0.035 \mathrm{GPM} / \mathrm{ft}^{2}$. In contrast, bundles 3 to 5 started at $0.030 \mathrm{GPM} / \mathrm{ft}^{2}$ and declined only slightly to $0.022 \mathrm{GPM} / \mathrm{ft}^{2}$. Out of all bundles, filter 4 appeared to show the most significant flux loss. Any flux convergence observed across filter bundles 1 to 5 likely results from flux loss as a result of reversible cake formation.

As discussed in Section 5.1.1, the suspected cause of filter flux divergence during the second test segment is speculated to be caused by depth fouling of the filters that occurs shortly after each backpulse. The frequent and repeated backpulse operations during the second $12 \mathrm{hrs}$ of testing provides significant opportunity for fines to access and plug the porous surface and sub-surface structures in the filter elements. The divergence observed in Low-Solids Scaling Test \#2 matches that observed in the first lowsolids test almost exactly, with the exception that filter bundle 3 appears to decline more severely in the second scaling test. As stated previously, the suspected propensity for fouling to occur in downstream filters (i.e., filters 3-5) is not well understood. Additional study of filter fouling dynamics (both on bench and engineering test scales) is recommended. 


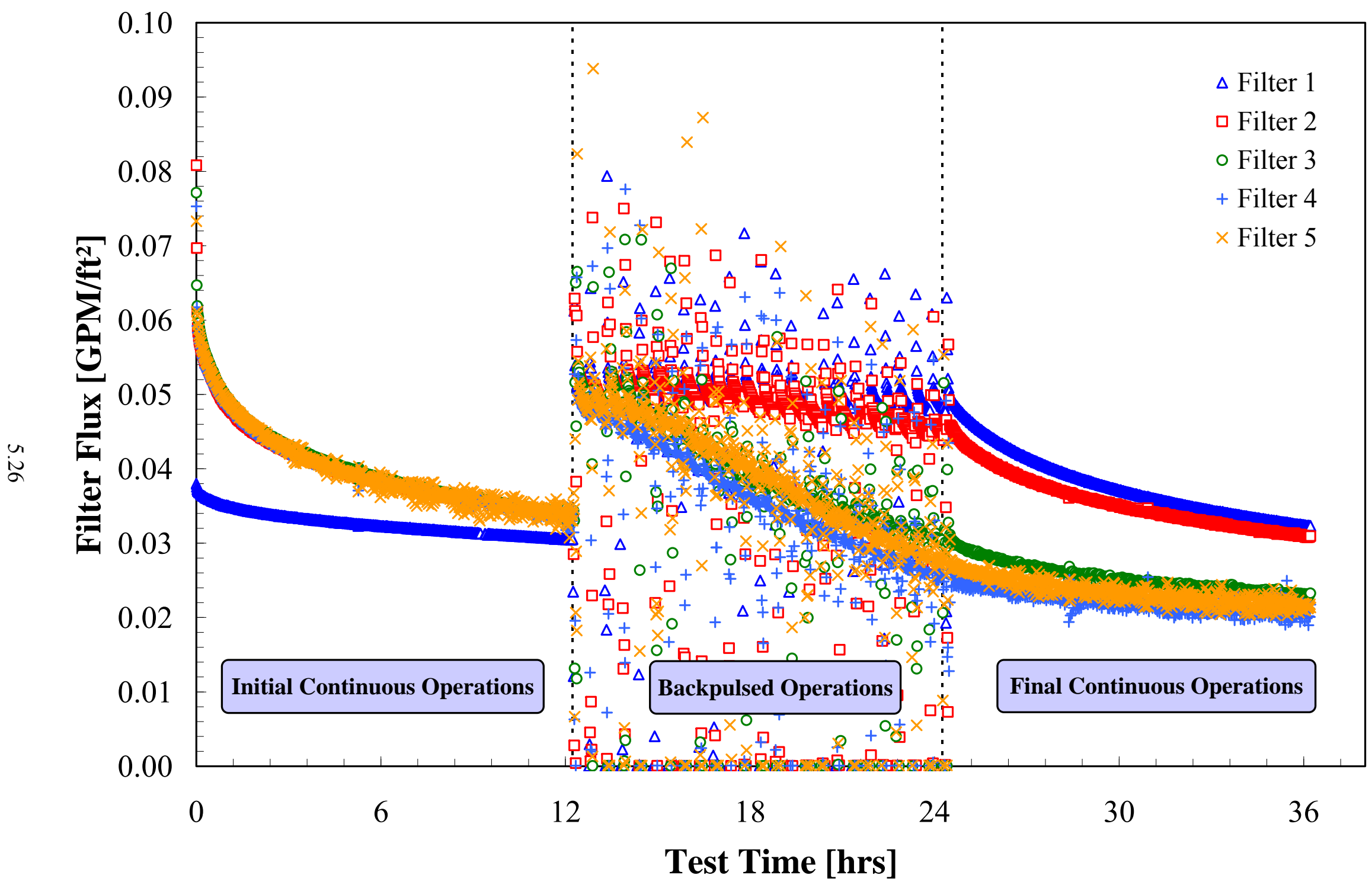

Figure 5.20. Individual Permeate Flux for PEP Filters (corrected for TMP and temperature variations) During Low-Solids Scaling Test \#2 
Figure 5.21 shows the total filter flux (i.e., the filter area averaged flux) for PEP during Low-Solids Scaling Test \#2. The filter flux is typical of that for continuous and backpulsed operations controlled by cake and depth fouling resistances. During the first $12 \mathrm{hrs}$, the flux steadily declines from an initial value of $0.060 \mathrm{GPM} / \mathrm{ft}^{2}$ down to $0.032 \mathrm{GPM} / \mathrm{ft}^{2}$ at $12 \mathrm{hrs}$. Backpulsed operations caused an initial flux recovery to $0.055 \mathrm{GPM} / \mathrm{ft}^{2}$; however, repeated backpulsing appears to lower the recovered total flux (as a result of apparent fouling on bundles 3 to 5). At the end of backpulsed operations, the recovered flux has decreased $0.040 \mathrm{GPM} / \mathrm{ft}^{2}$. Flux in the final 12-hr test segment steadily declines from an initial value of $0.040 \mathrm{GPM} / \mathrm{ft}^{2}$ to $0.030 \mathrm{GPM} / \mathrm{ft}^{2}$.

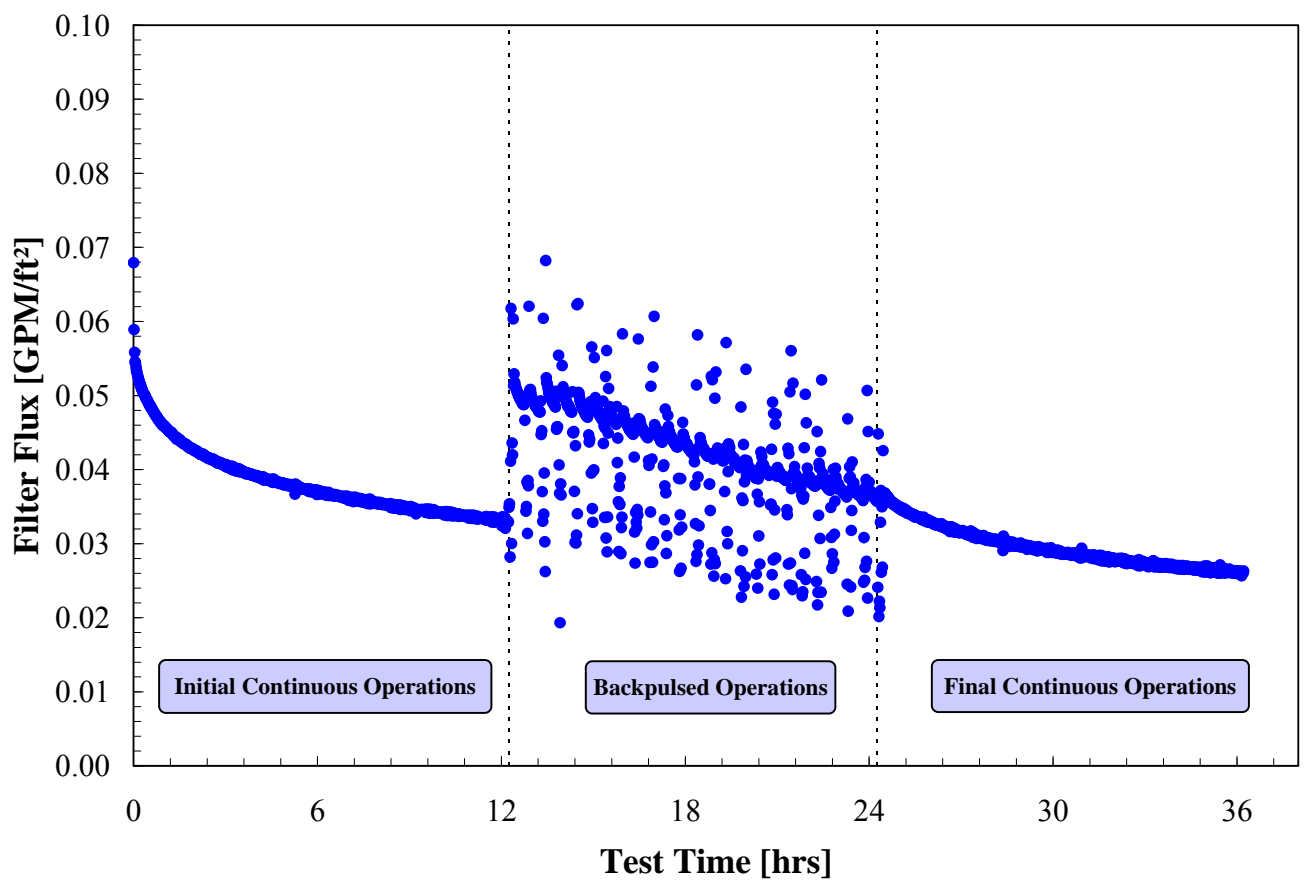

Figure 5.21. Total Permeate Flux for PEP Filters (normalized for TMP and temperature variations) During Low-Solids Scaling Test \#2

Like the first low-solids scaling test, both individual filter flux and total filter flux do not appear to reach a steady state value over the duration of the 36-hr PEP operations. From the current data, it is difficult to assess even the existence of a steady state flux as flux transience persists up to the very end of testing. Slow decays such as those shown in the current filtration curves can be associated with continued changes in the cake structure or slow plugging of the filter pores. Because of this, there is a risk that flux trends identified from the 36-hr duration tests do not continue (or change) over longer time scales. Additional testing that examines much longer periods of continuous (non-backpulsed) operations is required to determine the behavior of filter flux over long time periods and the existence of a steady state flux.

\subsubsection{CUF Operations}

The waste simulant slurry sample for parallel CUF testing was received from PEP on December 31, 2008. This slurry was loaded into the slurry reservoir on January 5, 2009. The second CUF low-solids filter scaling test (i.e., conditioning test) proceeded according to the test steps outlined in Section 4.2.2. Table 5.7 provides a summary of various test conditions. 
For the test, $5.28 \mathrm{~kg}$ of the $6.9-\mathrm{wt} \%$ waste simulant slurry was loaded into the CUF slurry reservoir. This yielded a slurry solids-to-filter surface area ratio of $1.4 \mathrm{~kg} / \mathrm{ft}^{2}$ for the scaling test. It should be noted that both CUF and PEP operations for Low-Solids Scaling Test \#2 employed the same slurry solids-tofilter surface area ratio of $1.4 \mathrm{~kg} / \mathrm{ft}^{2}$. Although matching slurry solids-to-filter surface area ratios is not entirely necessary (see Section 3.4.8), it does provide additional confidence when comparing flux at two different test scales. As such, the scaling results for Low-Solids Test \#2 should provide the best basis for a CUF and PEP scaling factor analysis out of the two scaling tests performed (i.e., the results for test \#2 are likely better than those for test \#1

Table 5.7. Test Conditions and Operational Parameters for CUF Low-Solids Scaling Test \#2

\begin{tabular}{ll}
\hline \multicolumn{1}{c}{ Parameter } & \multicolumn{1}{c}{ Value } \\
\hline Mass of slurry added to reservoir: & $5.28 \mathrm{~kg}$ \\
Ratio of slurry solids-to-filter surface area: & $1.4 \mathrm{~kg} / \mathrm{ft}^{2}$ \\
Process start time: & $01 / 05 / 2009$ 07:48 PST \\
Process end time: & $01 / 06 / 2009$ 20:01 PST \\
Elapsed time (duration): & 36.22 hours \\
Mixer impeller configuration: & Two impellers: a) 2-inch diameter propeller at the end of the \\
& shaft at one tank radius from the bottom b) 3-inch diameter, \\
& pitched, 3-blade turbine positioned 5 inches above the \\
& propeller \\
Mixer speed: & $450 \mathrm{rpm}$ \\
\hline
\end{tabular}

Once the initial target conditions of TMP $=40 \mathrm{psid}$ and AV $=15 \mathrm{ft} / \mathrm{s}$ were established for this test, the filtration system required only minor adjustments in operational parameters during the completion of the three segments of the conditioning test. CUF operational parameters during the second low-solids test are shown in Figure 5.22 to Figure 5.25.

Figure 5.22 shows the filter AV for CUF as a function of elapsed time. Apart from a few minor rate adjustments during the first 12-hr test period, the AV is stable and closely matches the target of $15 \mathrm{ft} / \mathrm{s}$. The average AV achieved over the course of testing is $15.0 \pm 0.6 \mathrm{ft} / \mathrm{s}(\mu \pm 2 \sigma)$. Deviations of the AV during the second 12-hr test segment correspond to reductions in slurry velocity during each filter backpulse. Figure 5.23 shows the filter TMP achieved during cold-CUF operations for Low-Solids Scaling Test \#2. For all test segments, TMP is relatively stable and closely matches the target TMP of 40 psid. Testing saw an average TMP of $40.2 \pm 0.4$ psid $(\mu \pm 2 \sigma)$.

Figure 5.24 shows the CUF filter APD measured during the second low-solids scaling test. It shows a relatively constant value of $2.8 \pm 0.5 \mathrm{psid}(\mu \pm 2 \sigma)$. Significant scatter in the measured APD derives from limited pressure sensor sensitivity. Figure 5.25 shows the CUF slurry reservoir temperature as a function of test time. An average slurry reservoir temperature of $25.0 \pm 0.3^{\circ} \mathrm{C}(\mu \pm 2 \sigma)$ was achieved and compares well to the target temperature of $25^{\circ} \mathrm{C}$. Slight negative deviations in the slurry temperature occur during backpulsing operations as a result of the temporary reduction in mechanical energy input from the pump as slurry flow and pressure are reduced during backpulse delivery. 


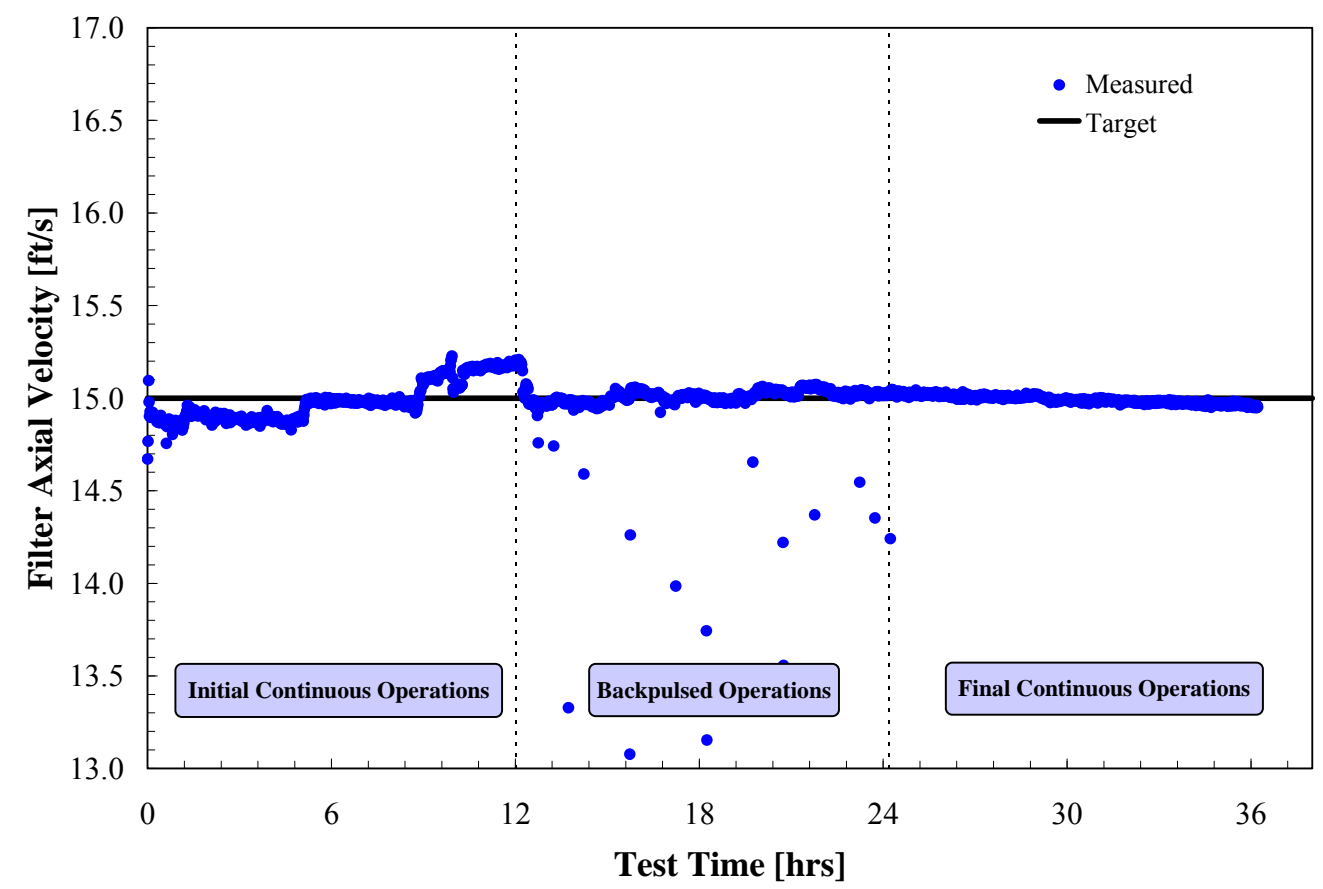

Figure 5.22. CUF Filter AV During Low-Solids Scaling Test \#2. The target AV was $15 \mathrm{ft} / \mathrm{s}$. An average velocity of $15.0 \pm 0.6 \mathrm{ft} / \mathrm{s}(\mu \pm 2 \sigma)$ was achieved in testing.

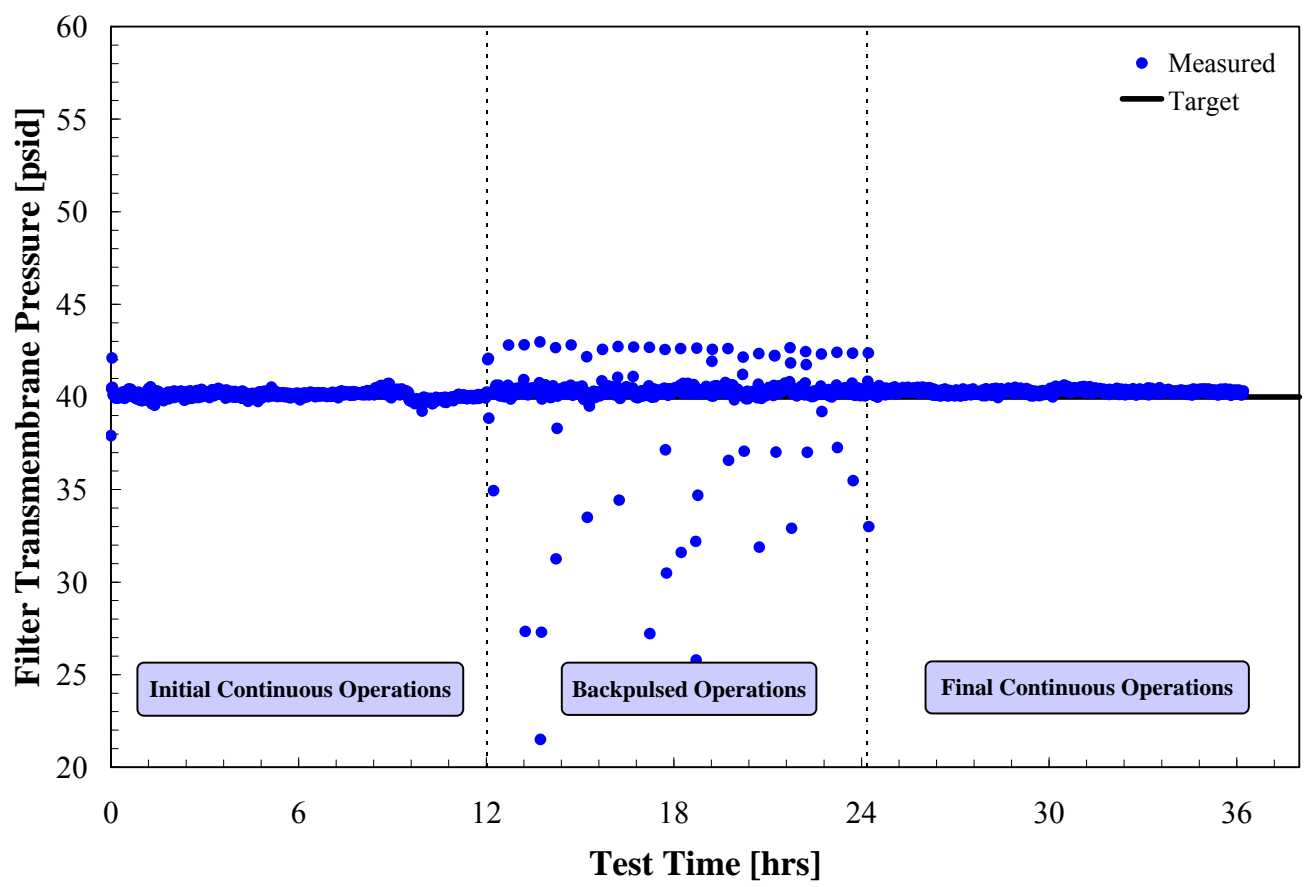

Figure 5.23. CUF Filter TMP During Low-Solids Scaling Test \#2. The target TMP was 40 psid. An average TMP of $40.2 \pm 0.4$ psid $(\mu \pm 2 \sigma)$ was achieved during continuous operations. 


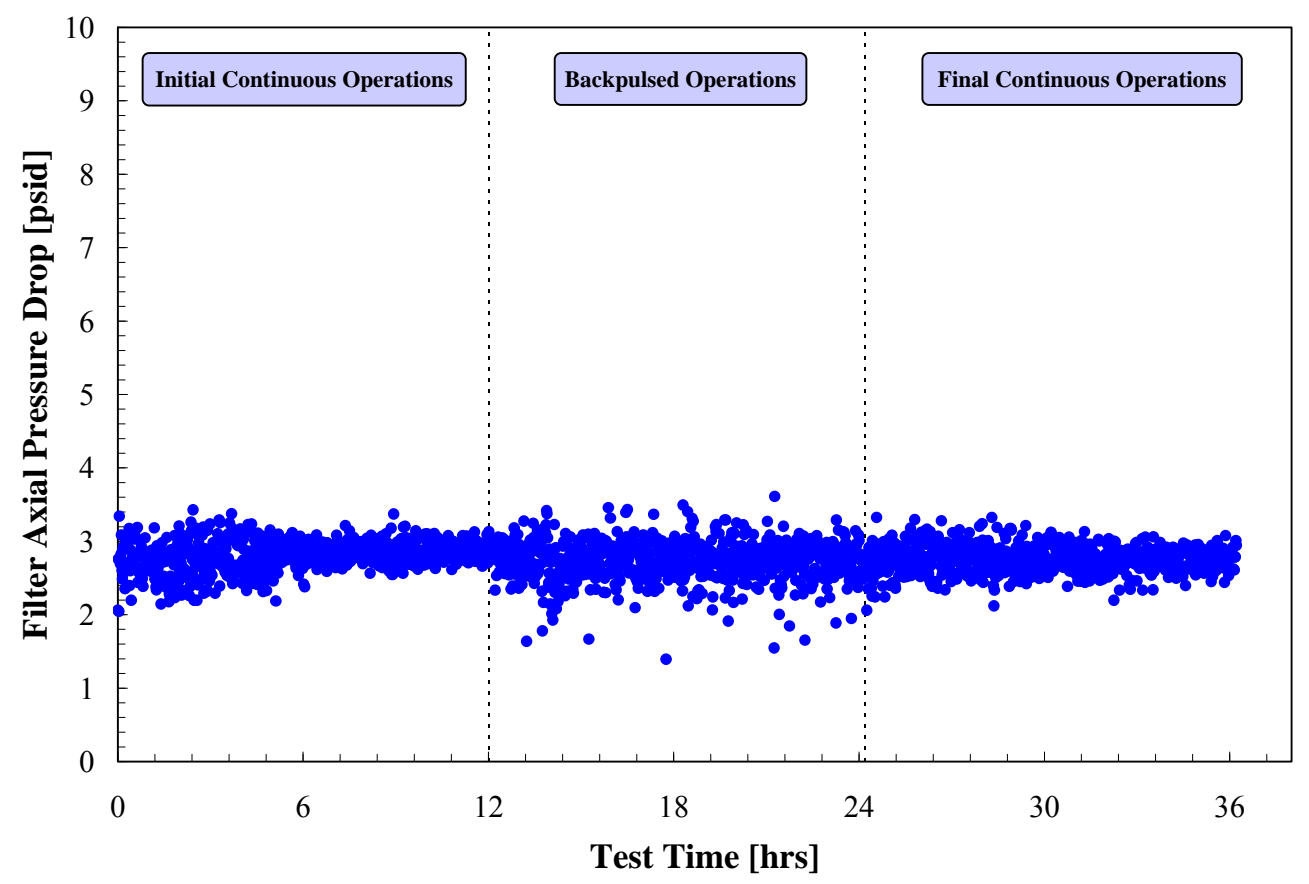

Figure 5.24. CUF Filter APD During Low-Solids Scaling Test \#2

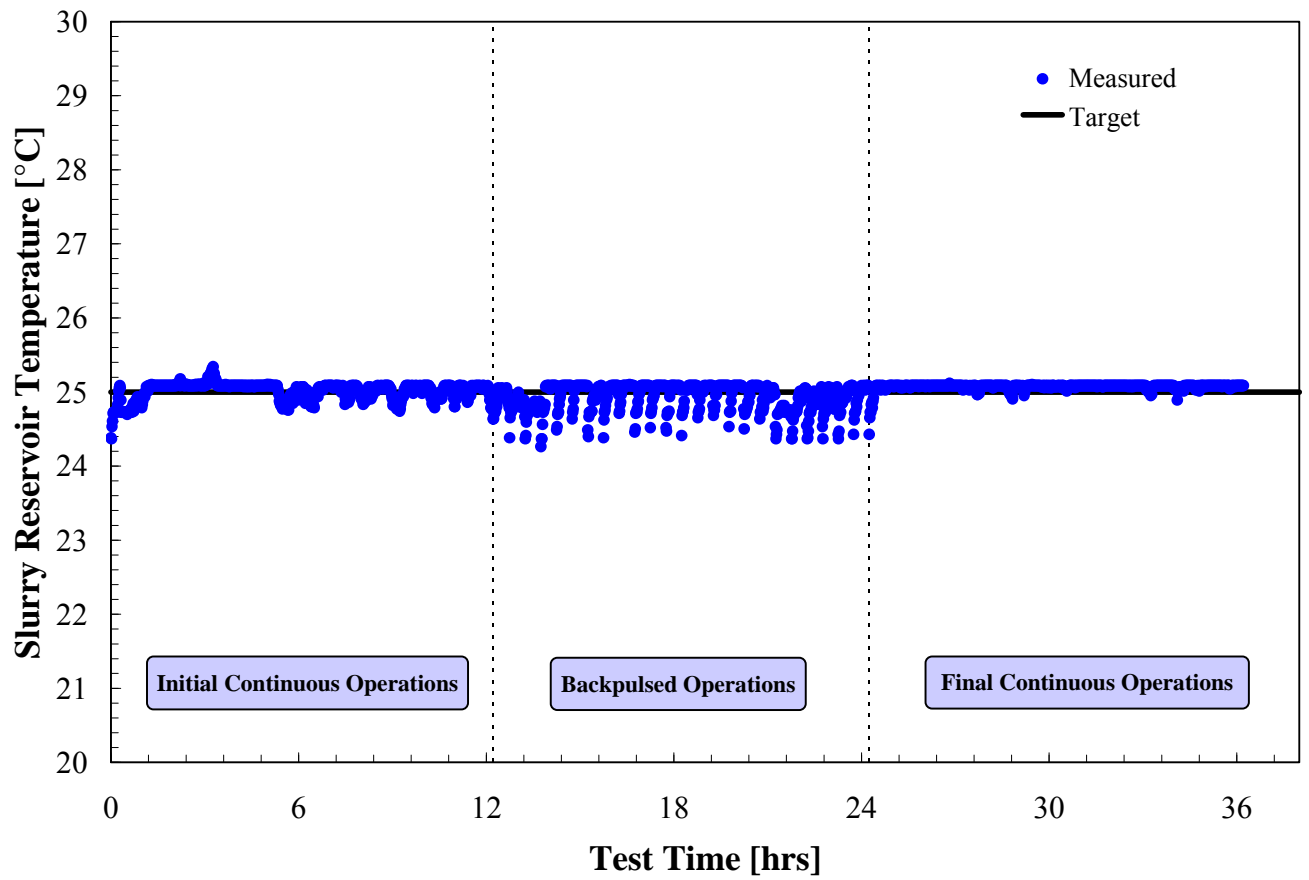

Figure 5.25. CUF Slurry Reservoir Temperature During Low-Solids Scaling Test \#2. The target temperature was $25^{\circ} \mathrm{C}$. An average temperature of $25.0 \pm 0.3^{\circ} \mathrm{C}(\mu \pm 2 \sigma)$ was achieved in testing. 
Figure 5.26 shows the CUF filter flux measured during Low-Solids Scaling Test \#2. Overall, the measured filter flux decreases from initial values near $0.060 \mathrm{GPM} / \mathrm{ft}^{2}$ down to $0.022 \mathrm{GPM} / \mathrm{ft}^{2}$ by the end of the test. As with CUF results from the first low-solids scaling test, the flux trends observed in Figure 5.26 are typical and evidence both cake-formation and depth-fouling. Specifically, the flux trends in the first $12 \mathrm{hrs}$ appear to be dominated by cake-formation (and possibly filter depth fouling). Here, the filter flux decreases from an initial value of $0.060 \mathrm{GPM} / \mathrm{ft}^{2}$ down to $0.025 \mathrm{GPM} / \mathrm{ft}^{2}$. The initial backpulse in the second 12-hr segment of testing restores much (but not all) of the original filter flux. Relative to the initial flux of the $0.06 \mathrm{GPM} / \mathrm{ft}^{2}$, the flux recovered on the first backpulse of $0.05 \mathrm{GPM} / \mathrm{ft}^{2}$ is suggestive of irreversible filter depth fouling. During backpulsing operations, the recovered flux continues to decline. At the end of backpulsed operations, filter fouling has reduced the recovered CUF flux to 0.038 $\mathrm{GPM} / \mathrm{ft}^{2}$. It should be reiterated that the recovered fluxes reported here represent 1-minute averages, that the actual flux variation may be larger when $1-\mathrm{Hz}$ data are considered, and that the maximum recovered flux is also likely larger (in magnitude) relative to the 1-minute averaged flux. In the final test segment, the CUF flux declines steadily under non-backpulsed operations. The decline is characteristic of filter cake formation (and possibly of slowed filter depth fouling). The final flux at the end of testing is 0.022 $\mathrm{GPM} / \mathrm{ft}^{2}$.

From this test sequence of limited duration, it appears that the flux continues to decrease with time and is asymptotically approaching a minimum filter flux for a given set of process conditions. Further testing would be necessary to determine what minimum (i.e., steady-state) value the flux approaches.

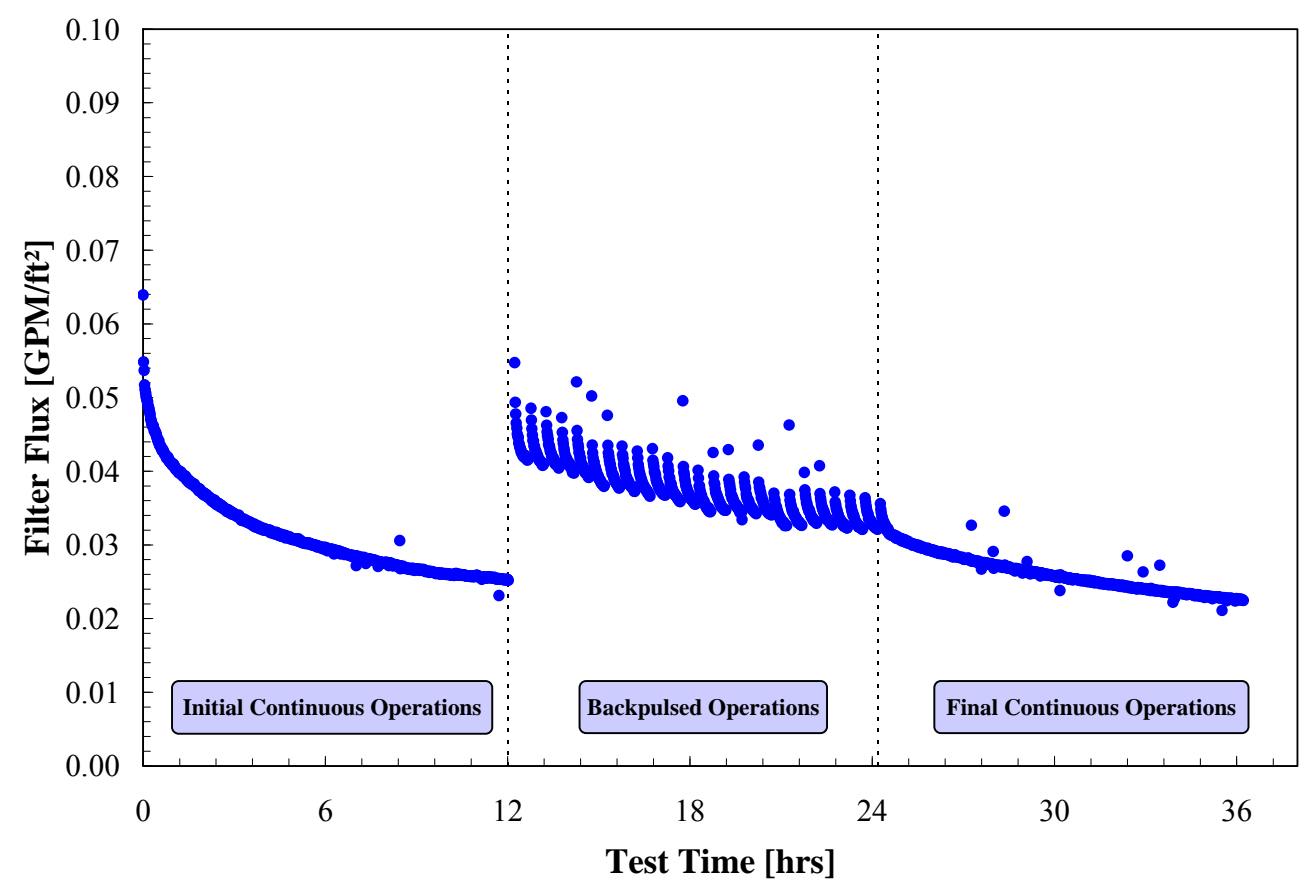

Figure 5.26. CUF Filter Flux (corrected for TMP and temperature variations) During Low-Solids Scaling Test \#2 


\subsubsection{Analysis of Filter Scaling for Low-Solids Test \#2}

As with the first low-solids scaling test, flux data measured during Low-Solids Scaling Test \#2 were compared to determine the PEP to CUF filter flux scaling factor. Because these two studies were performed at a similar solids-to-filter surface area ratio of $1.4 \mathrm{~kg} / \mathrm{ft}^{2}$, comparison of filter flux between the two different test scales should be appropriate. It should again be noted that PEP and CUF filters have significantly different test histories. The PEP filter bundles were used only for limited water and simulant functional testing before Low-Solids Scaling Test \#2. In contrast, the CUF filters were used for a significant number of tests throughout Calendar Year 2008 (see discussion Section 4.1). Cleaning CUF and PEP filters with acid solution may not eliminate differences caused by filter history. As such, some of the differences in filter behavior may be attributable to differences in the state of initial filter cleaning. Unfortunately, filter cleanliness effects cannot be separated from scaling effects in the current analysis.

Figure 5.27 shows the filter flux for individual PEP filters and that for the CUF filter. During the first $12 \mathrm{hrs}$ of operation, the CUF filter flux falls below that of the PEP filter bundles with exception of PEP filter bundle 1. Divergent behavior for the first PEP filter bundle can be ignored as it appears to have resulted from ineffective backpulsing of filter bundle 1. Filter backpulsing operations do not appear to foul the CUF flux as dramatically as the downstream PEP filter bundles. Thus, the CUF flux appears to fall just above the flux for PEP filter bundle 3 at the conclusion of backpulse operations. In the final test segment, upstream PEP filter bundles still show high flux relative to the CUF flux; however, the CUF flux compares well with the flux of downstream filters. Overall, the CUF flux does not provide a representative measure of individual PEP filter performance for Low-Solids Scaling Test \#2. 


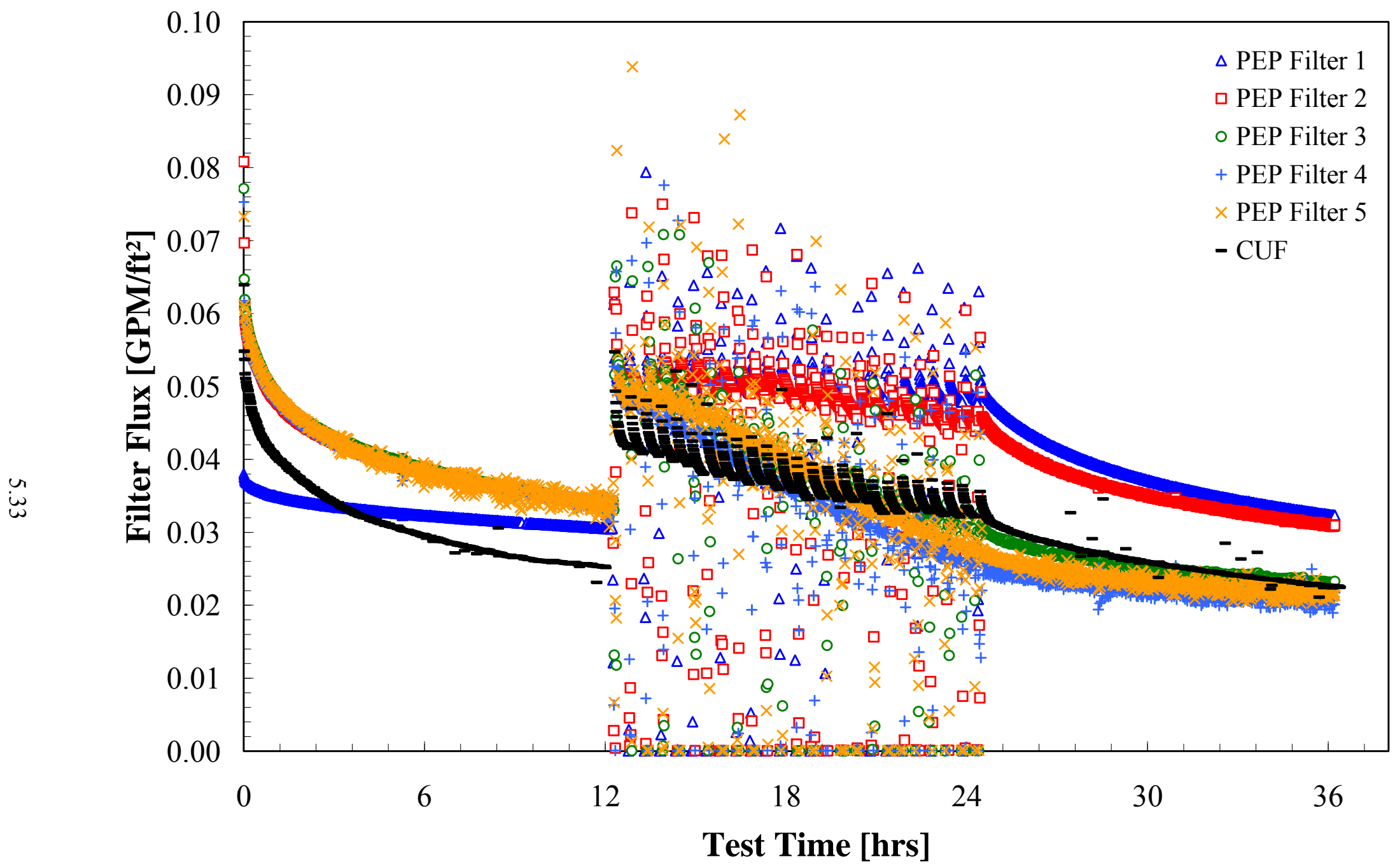

Figure 5.27. Comparison of CUF Filter Flux to Individual PEP Filter Bundle Flux During Low-Solids Scaling Test \#2. All filter fluxes have been corrected for TMP and temperature variations. The CUF test time scale has been modified to better align the periods of continuous and backpulsed filtration for CUF and PEP. 
Figure 5.28 compares the CUF flux to the total PEP filter flux. It should be noted that the total (areaaverage) PEP flux in Figure 5.28 is likely lower by up to $8 \%$ at the start of filtration from the "true" value (i.e., that corresponding to a clean, cake-free filter) by the inclusion of the filter flux from bundle 1 (which appears to have been impacted by cake formation). The lowering in the area-averaged (total) PEP flux declined over the course of the first $12 \mathrm{hrs}$ because the flux on filter 1 and filter 2 to 5 converges (as flux approaches steady-state). As with the first low-solids scaling test, the CUF flux is lower than the corresponding PEP flux during the first $12 \mathrm{hrs}$ of operations (i.e., when both filters are relatively unconditioned by the waste simulant slurry). The filter fluxes for the two test scales appear to converge during backpulsing operations and closely track one another in the final 12-hr test segment. Even after filter conditioning, the CUF flux is somewhat lower than that measured in the PEP.

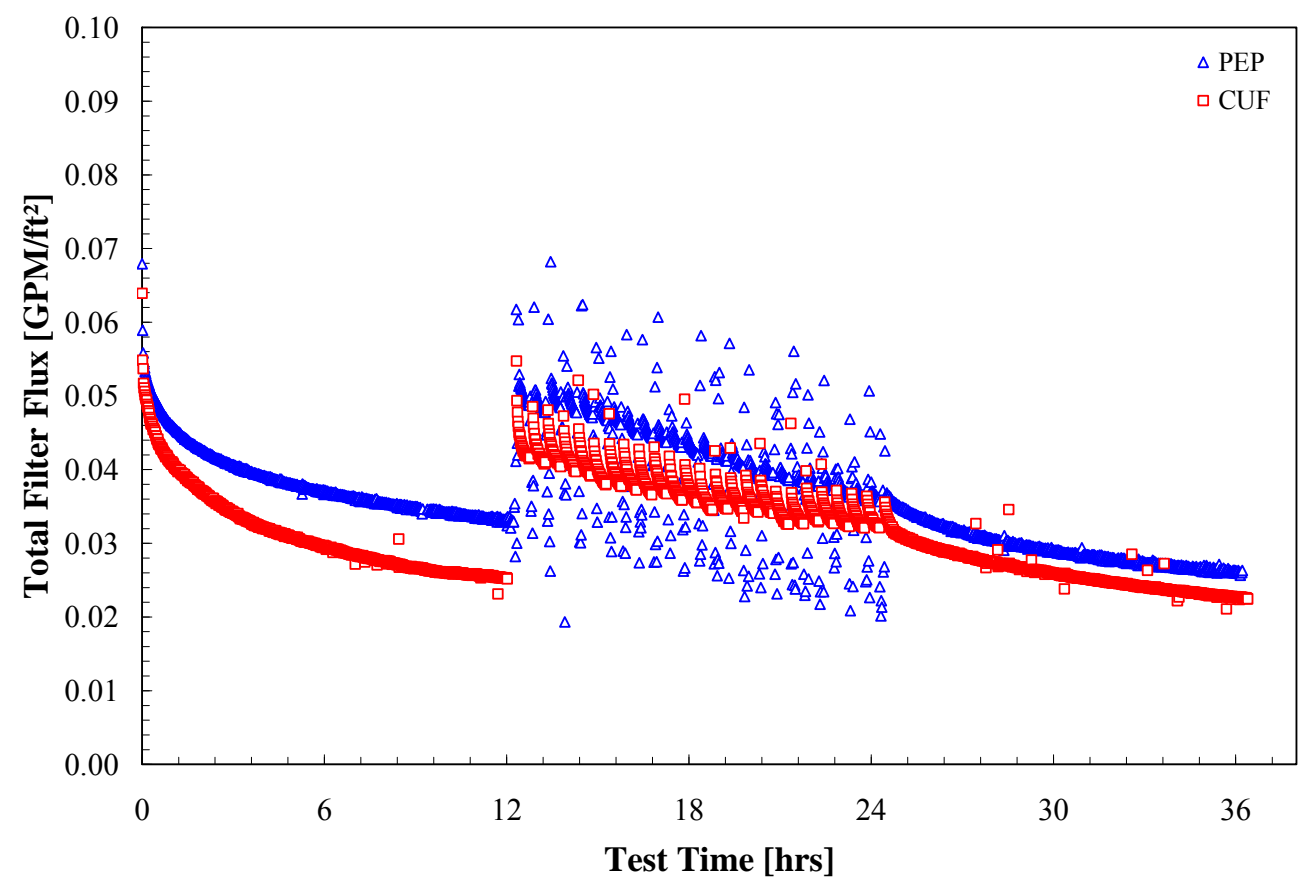

Figure 5.28. Comparison of CUF Filter Flux to Total PEP Filter Flux During Low-Solids Scaling Test \#2. Both filter fluxes have been normalized for TMP and temperature variations. The CUF test time scale has been modified to better align the periods of continuous and backpulsed filtration for CUF and PEP.

For completeness, both individual scaling factors and a total scaling factor are derived from the data in Figure 5.27 and Figure 5.28. As before, any differences in test time between the start of each 12-hr period of operations in CUF and PEP have been corrected. The results for Low-Solids Scaling Test \#2 have been subjected to a similar averaging regimen as described in Section 5.1.3 to create reference fluxes for scaling factor analysis as a function of nominal test time. Individual scaling factors (i.e., PEP to CUF scaling factors for filters 1 to 5) are shown in Figure 5.29. During the first 12-hr segment, the scaling factors typically range from 1.2 to 1.4 and indicate that the CUF under predicted the PEP filter flux. It should be noted that the relatively low scaling factor for filter $1(0.8$ to 1.2$)$ results from the ineffective backpulse at the start of the scaling test. At the start of backpulsing operations, the scaling factors drop from $\sim 1.4$ to 1.0-1.2. Subsequent backpulse operations evidence a divergence in the PEP to CUF scaling factors as an apparent result of non-uniform depth fouling. Scaling factors for upstream filters (1 and 2) 
are $\sim 1.4$, while scaling factors for downstream filters ( 3 to 5 ) range from 0.8 to 1.0 . The spread in scaling factors supports the earlier assertion that estimates of individual filter bundle flux derived from CUF flux will be approximate at best (at least when using only a single value for scaling factor). Based on the excellent agreement between CUF flux and total (area-averaged) PEP flux, the use of a scaling factor based on total flux would provide a better estimate of scaled flux.

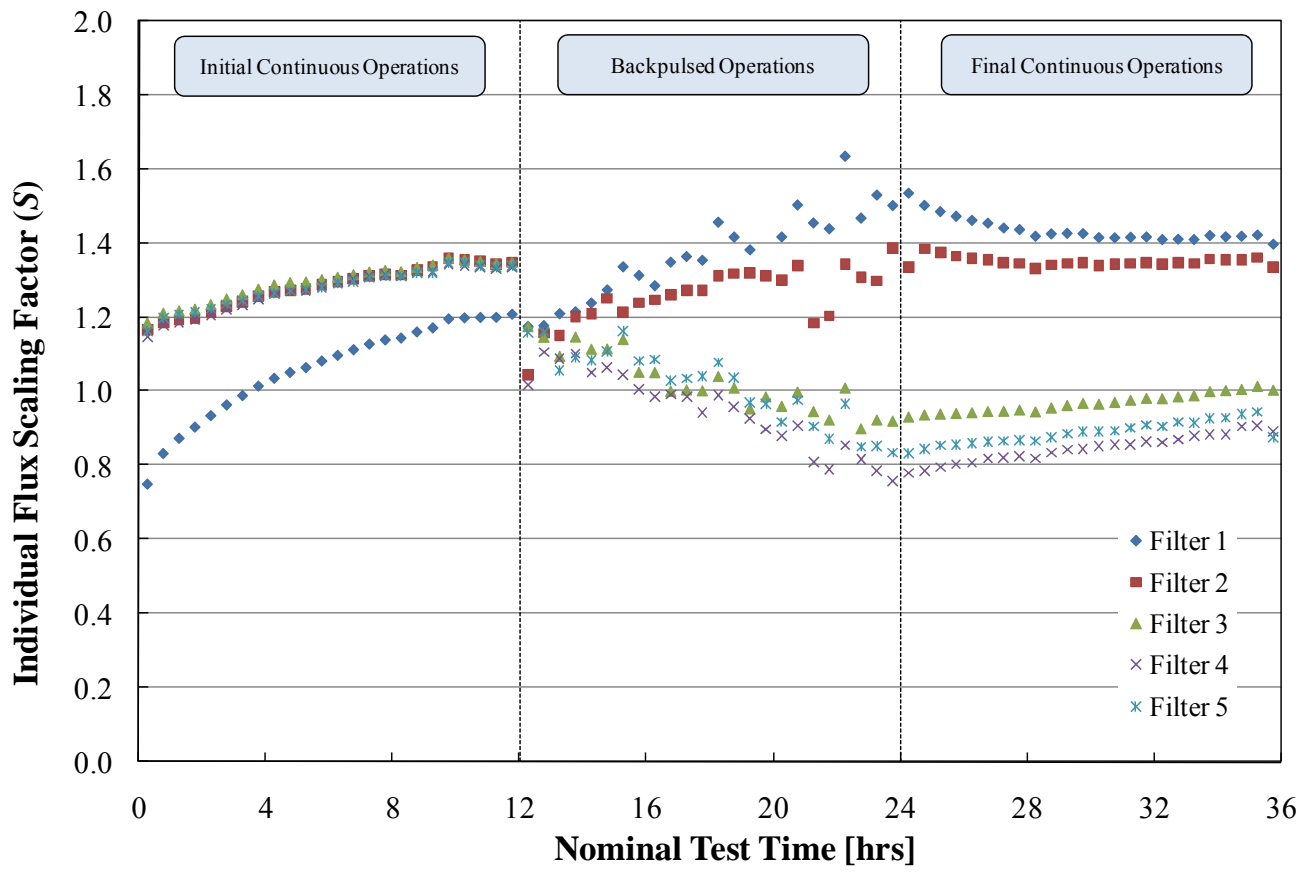

Figure 5.29. PEP to CUF Scaling Factor all Five PEP Filters as a Function of Time for Low-Solids Scaling Test \#2. Each scaling factor value has an associated error of approximately $8 \%$.

Figure 5.30 shows the total scaling factor for PEP to CUF operations. During the first 12-hr period of operations, the total scaling factor ranges from $\sim 1.1$ to 1.3 . It should be noted that the scaling factor in this 12-hr period reduced slightly by inclusion of flux from filter bundle 1 . The error associated with inclusion of filter bundle 1 flux is comparable to that associated with process variation $(\sim 8 \%)$ at the beginning of the test and decreases thereafter. At the start of backpulse operations, the scaling factor falls between 1.1 and 1.2 and does not change substantially throughout the remaining test operations. The results in Figure 5.30 suggest that, when conditioned against a similar waste slurry simulant, the coldCUF filtration system slightly under predicts fluxes achievable on the PEP test scale. Because the scaling factor is relatively constant around $\sim 1.1$ throughout the latter half of the test, the CUF also appears able to provide a representative measurement of transient effects in waste filtration. It should be noted that this final observation is limited by the fact that for the current tests filtration steady state was never achieved. As such, there is a potential that when considered on longer time scales, CUF and PEP may scale differently than observed in Figure 5.30. Further tests to assess long-term filter transience are recommended.

Table 5.8 provides a summary of scaling factors (as individual and total scaling factors) for the initial continuous 12-hr period, the backpulse 12-hr period, and the final continuous 12-hr period of operations. The results shown in Table 5.8 are test segment averages of the scaling factors shown in Figure 5.29 and Figure 5.30. As stated previously, the most appropriate results correspond to the total scaling factors for 
backpulsed and final continuous operations $(1.1 \pm 0.1$ for both test periods). The errors associated with these results suggest that the PEP to CUF scaling factor is near or slightly larger than one. To provide a conservative estimate for process scaling, a scaling factor of 1.0 is recommended (based on the current test results). It should also be noted that the results are statistically similar to those derived for LowSolids Scaling Test \#1.

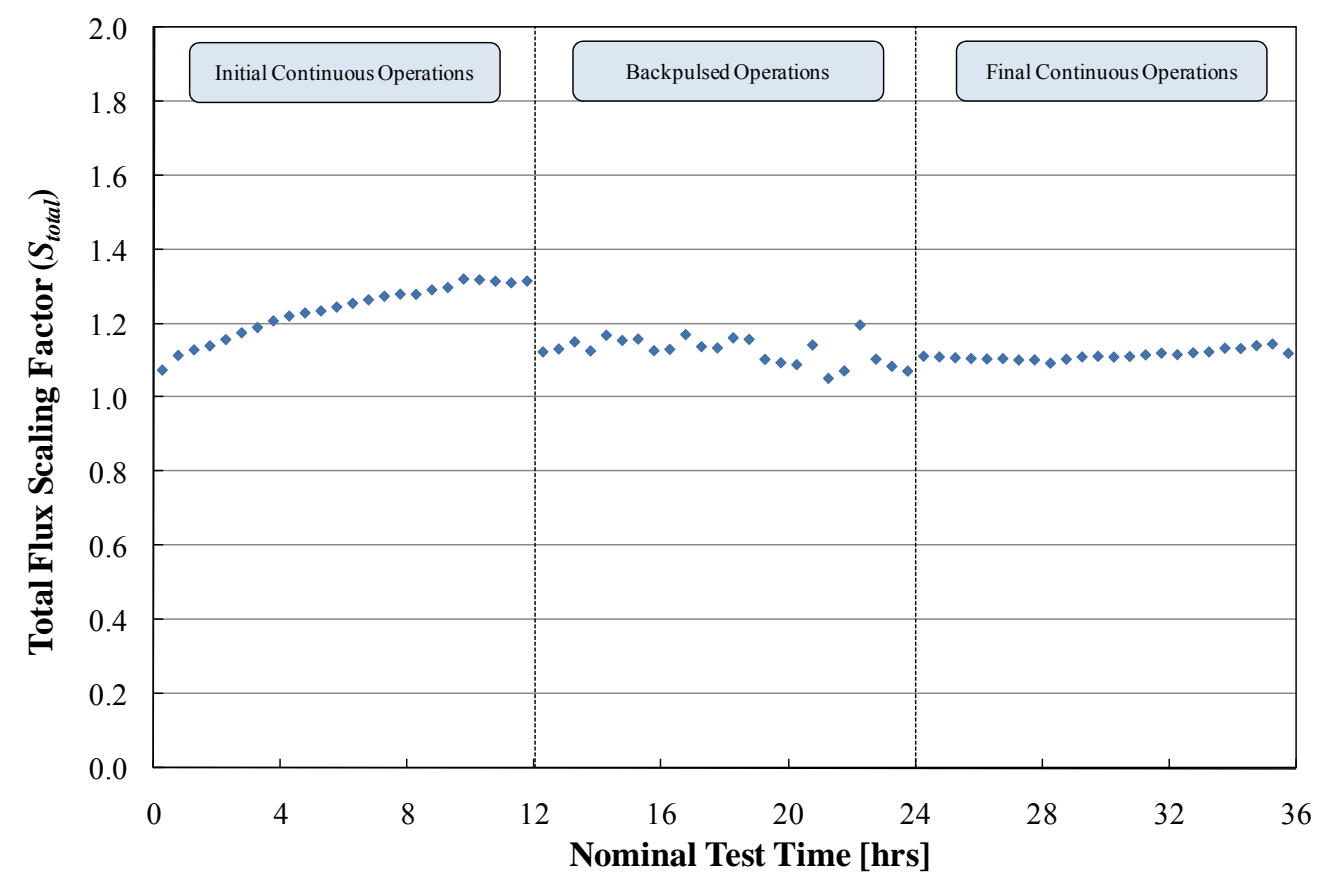

Figure 5.30. Total PEP to CUF Scaling Factor as a Function of Time for Low-Solids Scaling Test \#2. Each scaling factor value has an associated error of approximately $8 \%$.

Table 5.8. $\quad$ Average PEP to CUF Scaling Factors for Filter Flux as a Function of Filter and as a Function of Process Operation. Scaling factors were derived from Low-Solids Scaling Test \#2. Values are reported as the mean value; uncertainty is twice the sample standard deviation.

\begin{tabular}{lcccccc}
\hline & \multicolumn{5}{c}{ Average Scaling Factor $\left(S_{i}\right)$} \\
\cline { 2 - 7 } Process Operation & Filter 1 & Filter 2 & Filter 3 & Filter 5 & Filter 6 & Total \\
\hline Initial Continuous & $1.1 \pm 0.1$ & $1.3 \pm 0.1$ & $1.3 \pm 0.1$ & $1.3 \pm 0.1$ & $1.3 \pm 0.1$ & $1.2 \pm 0.1$ \\
Backpulsed & $1.4 \pm 0.1$ & $1.3 \pm 0.1$ & $1.0 \pm 0.1$ & $0.9 \pm 0.1$ & $1.0 \pm 0.1$ & $1.1 \pm 0.1$ \\
Final Continuous & $1.4 \pm 0.1$ & $1.3 \pm 0.1$ & $1.0 \pm 0.1$ & $0.8 \pm 0.1$ & $0.9 \pm 0.1$ & $1.1 \pm 0.1$ \\
\hline
\end{tabular}

As a final comparison for the low-solids scaling tests, Figure 5.31 and Figure 5.32 present the filter fluxes comparing the filter flux measured in Low-Solids Test \#2 to that measured in Low-Solids Scaling Test \#1 for PEP and CUF, respectively. The comparison for both CUF and PEP show that the filter flux measured during the second low-solids scaling test was lower than that measured in the first test across all test segments. This is expect based on the analytical results, as the slurry used for Low-Solids Scaling Test \#1 had a lower dissolved solids content and supernate viscosity $(25.3 \mathrm{wt} \%$ and $2.4 \mathrm{mPa} \cdot \mathrm{s}$, respectively) than that employed for Low-Solids Scaling Test \#2 (27.1 wt\% and $2.8 \mathrm{mPa} \cdot \mathrm{s}$, respectively). 
A lower dissolved solids concentration is expected to yield a lower permeate viscosity and a corresponding increase in filter flux.

The CUF flux shows similar trends with test time. In fact, the CUF filter flux curves for test \#1 and \#2 track each other closely. A correction for permeate viscosity differences would likely cause the curves to nearly coincide. In contrast, the PEP curves do not track each other closely. For the first 12-hr test segment, part of the difference in flux between PEP Low-Solids Test \#1 and Test \#2 derives from the inadequate backpulse of filter bundle \#1 at the start of testing. Specifically, the backpulse was not sufficient to remove cake from filter bundle \#1, and as a result, the total filter flux for the first segment of Low-Solids Test \#2 is lower than it would have been had the initial backpulse adequately cleaned the filter. However, the difference caused by this inclusion is comparable to the error associated with process variation (it becomes small toward the end of the first 12-hr continuous period of operations). Beyond the first 12 hrs, the PEP filter flux for test \#1 and test \#2 track more closely; however, direct comparison for filter flux in the backpulsed (second 12-hr) segment was complicated by a mismatch in the times when the filters were backpulsed. On the other hand, the difference in PEP flux at the end of the final 12-hr test segment appears to approach that observed for CUF in Figure 5.32.

In short, Figure 5.31 and Figure 5.32 appear to indicate that the time-dependent flux behavior is reproducible for CUF. Differences in execution of low-solids in test \#1 and test \#2 prevent making the same statement with similar certainty for PEP filtration. However, flux measurements for the final 12-hr segment of the PEP conditioning operations appear to support the conclusion of reproducibility. When considered against the plots comparing CUF to PEP flux (i.e., Figure 5.13 and Figure 5.28), the differences between tests at the same scale are similar to the differences in the flux behavior observed across bench and engineering scales. The consequence of this observation for the current report is that a significant portion of the scaling factor variation observed in Table 5.4 and Table 5.8 may be associated with typical experimental reproducibility. Since the uncertainty reported with the scaling factors for lowsolids tests only considers process sensor variation, experimental reproducibility may not be fully captured. 


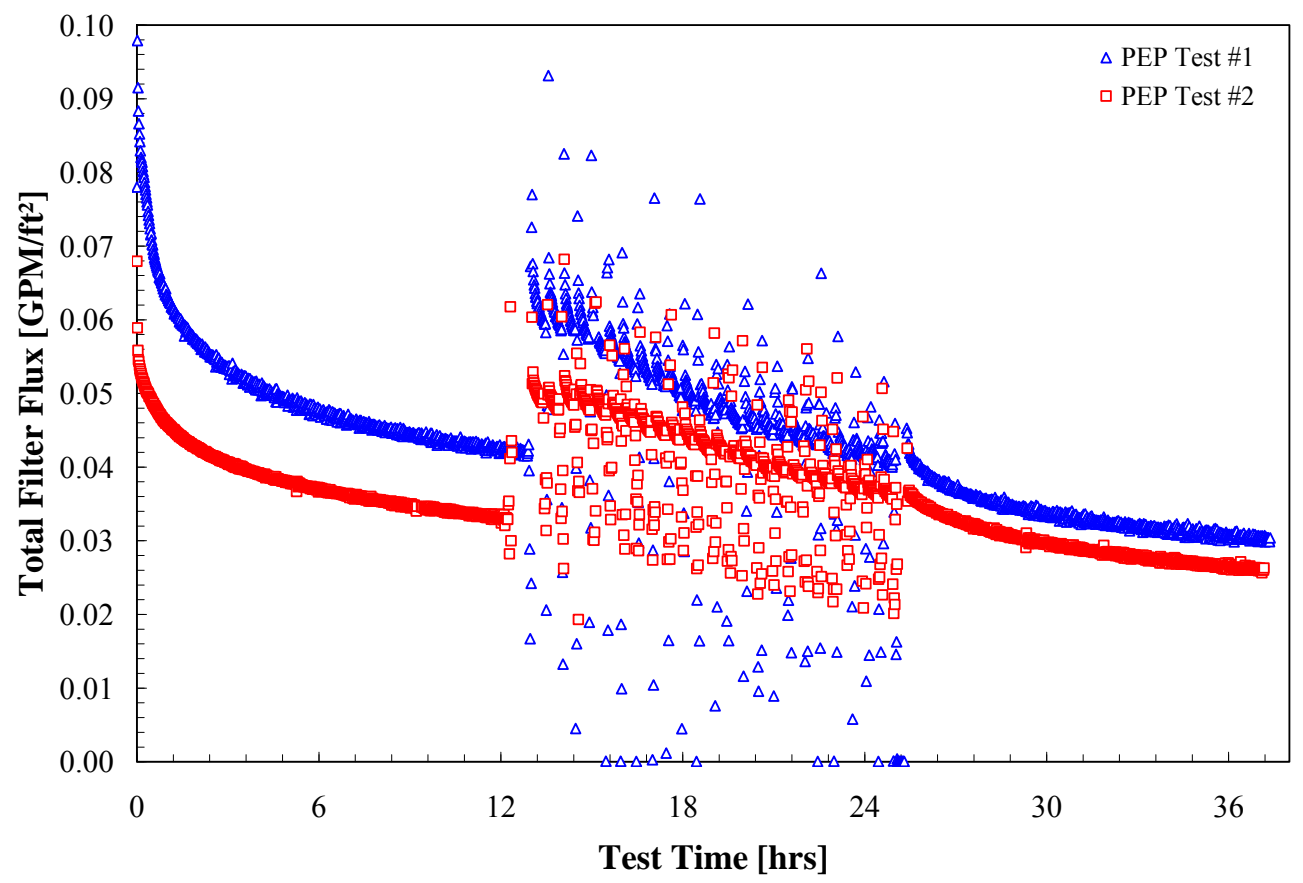

Figure 5.31. Comparison of Total PEP Filter Flux for Low-Solids Scaling Tests \#1 and \#2. The test time scale for PEP Test \#2 has been modified to better align the periods of continuous and backpulsed filtration for CUF and PEP.

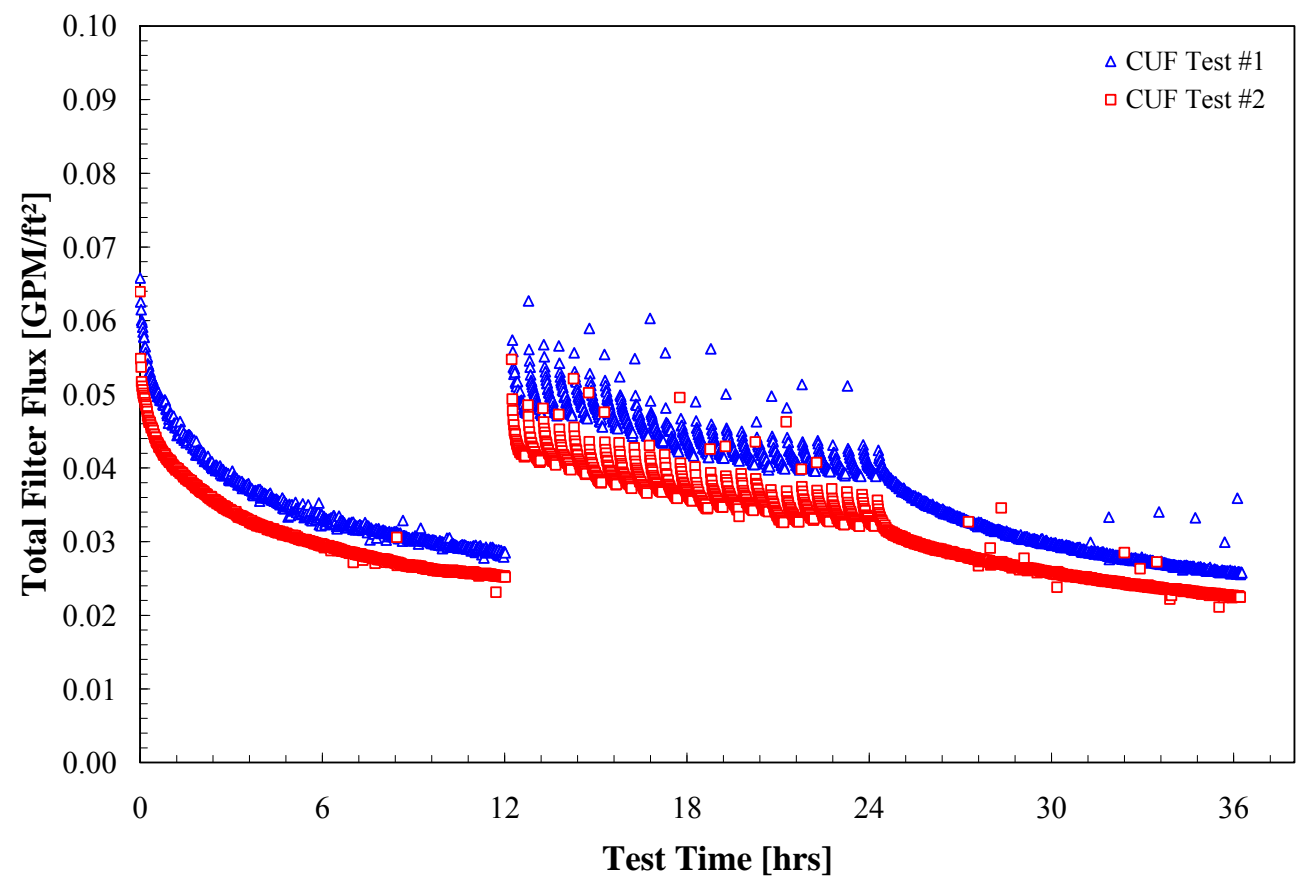

Figure 5.32. Comparison of Total CUF Filter Flux for Low-Solids Scaling Tests \#1 and \#2. The test time scale for CUF Test \#1 has been modified to better align the periods of continuous and backpulsed filtration for CUF and PEP. 


\subsection{Test Results for the High-Solids Scaling Test}

PEP operations for the High-Solids Scaling Test were conducted from $0220 \mathrm{hrs}$ to $0608 \mathrm{hrs}$ March $21^{\text {st }}, 2009$, PDT. PEP process data were recorded by the PEP DAS at a frequency of $1 \mathrm{~Hz}$. For subsequent analyses, all relevant $1 \mathrm{~Hz}$ data (i.e., those over the process times of interest) were pulled and averaged over 1-minute intervals. The sample for bench-scale testing (ID\# A 02AML 022 XX 2467 CUF 4) was taken at $0130 \mathrm{hrs}$ March $21^{\text {st }}, 2009$. This sample was delivered to APEL for cold CUF testing. The sampling time occurred before the start of the high-solids test; as such, there may be a difference in the initial UDS concentrations of the CUF and PEP test slurries. This difference does not impact test results because the two systems will be compared as functions of UDS concentrations (rather than on a time basis as for the low-solids scaling tests discussed in previous sections). Two separate parallel CUF tests were performed: an initial high-solids dewatering test and a repeat of that dewatering test. The initial test operations were conducted from $1320 \mathrm{hrs}$ to $1703 \mathrm{hrs}$ March $25^{\text {th }}, 2009$, PDT. The repeat test operations were conducted from $0912 \mathrm{hrs}$ to $1435 \mathrm{hrs}$ March $27^{\text {th }}, 2009$, PDT. CUF data were recorded by the CUF DACS at a frequency of $0.4 \mathrm{~Hz}$. The CUF DACS then averaged all $0.4 \mathrm{~Hz}$ data over 1-minute process intervals. For all CUF data analyses, the 1-minute data averages were employed. The operations and scaling results associated with these tests are discussed in detail in the following sections.

\subsubsection{PEP Operations}

The waste simulant slurry used for PEP high-solids dewatering operations consisted of a mixture of leached and washed solids from Integrated Test A and B test operations (see Section 4.3 and/or TP-WTPRPP-506 ${ }^{(a)}$ for details). After slurry solids preparation and dilution, the circulating mass in Tank T02A and the filter loop was composed of approximately $1420 \mathrm{~kg}$ of a $15.4-\mathrm{wt} \%$ UDS waste simulant slurry (based on a material balance of Tank T02A and the filter loop and regression of available UDS data). This resulted in a slurry solids mass to filter surface area ratio of $13.9 \mathrm{~kg} / \mathrm{ft}^{2}$. This falls in the range of the slurry solids-to-filter surface area ratio anticipated for WTP (1.7 to $16 \mathrm{~kg} / \mathrm{ft}^{2}$ - see Section 5.1.1).

A summary of key slurry properties is listed in Table 5.9. No rheology measurements were made on the High-Solids Scaling Test slurry. In addition, UDS and bulk density are not listed in Table 5.9 as dewatering operations increase both these slurry properties over time. The evolution of UDS as a result of dewatering operations is shown in Figure 5.33 where results for both actual UDS measurements (blue circles) and UDS estimations based on material balance equations (solids line) are shown as a function of test time elapsed. The slurry bulk density varied from $1.13 \mathrm{~kg} / \mathrm{L}$ to $1.25 \mathrm{~kg} / \mathrm{L}$. Table 5.10 shows the operational parameters for Tank T02A PJM sparging systems that were achieved in the High-Solids Scaling Test. All test conditions were achieved to within target tolerances.

(a) GB Josephson, OP Bredt, JK Young, and DE Kurath. 2009. Pretreatment Engineering Platform (PEP) Testing (Phase I). TP-RPP-WTP-506, Rev. 0.4, Pacific Northwest National Laboratory, Richland, Washington. 
Table 5.9. PEP Slurry Properties for the High-Solids Scaling Test

\begin{tabular}{lcl}
\hline Property & Measured Value & Units \\
\hline Approximate Slurry Mass Tested & 1420 & $\mathrm{~kg}$ \\
Slurry Solids-to-Filter Area Ratio & 13.9 & $\mathrm{~kg} / \mathrm{ft}^{2}$ \\
Permeate Density & $1.00 \pm 0.05$ & $\mathrm{~kg} / \mathrm{L}$ \\
Solids Concentrations & & \\
$\quad$ Dissolved Solids In Permeate & $0.79 \pm 0.08$ & $\mathrm{wt} \%$ \\
$\quad$ Centrifuged Solids & $46.4 \pm 1.4$ & $\mathrm{wt} \%$ \\
\hline
\end{tabular}

Table 5.10. Operation Conditions Achieved for Tank T02A PJM and Sparging Systems During the High-Solids-Scaling Test

\begin{tabular}{lcc}
\hline Parameter & Target & Actual \\
\hline Tank T02A PJM Jet Velocity & $12 \pm 2 \mathrm{~m} / \mathrm{s}$ & $11 \mathrm{~m} / \mathrm{s}$ \\
Tank T02A PJM Cycle Time & $20 \pm 1 \mathrm{~s}$ & $20.3 \mathrm{~s}$ \\
Tank T02A PJM Stroke Length & $31.5-6 /+3 \mathrm{in}$. & 33.6 in. $(89 \%)$ \\
Tank T02A Steam Ring Purge Flowrate & Off & Off \\
Tank T02A Upper Air Sparger Flowrate & Off & Off \\
Tank T02A Total Lower Air Sparger Flowrate & Off & Off \\
Number of Filter-Loop Bundles & 1 & 1
\end{tabular}

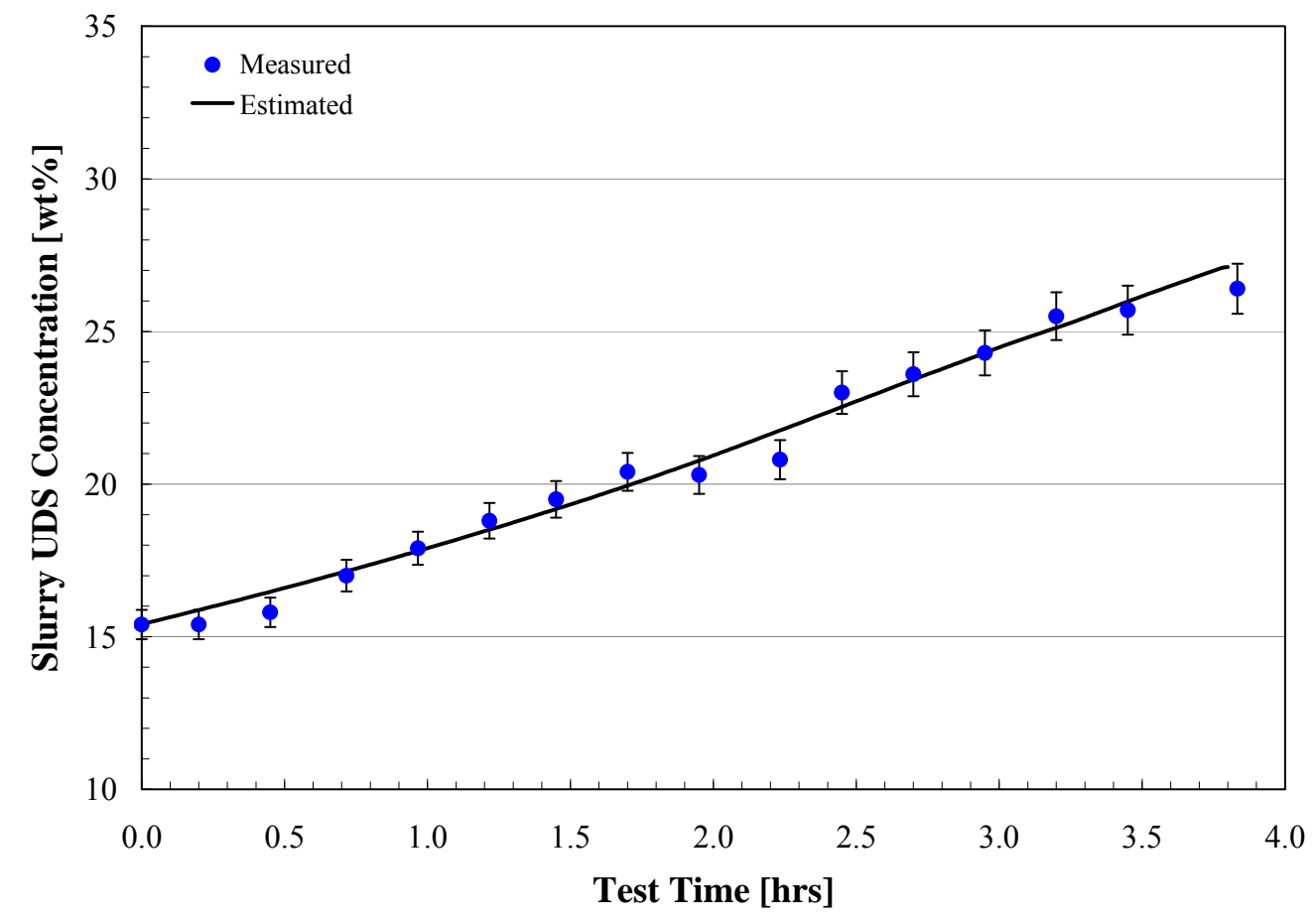

Figure 5.33. Slurry UDS Concentrations Tested During the PEP High-Solids Scaling Test. Results for both actual UDS measurements (blue circles) and UDS estimations based on material balance equations (solids line) are shown as a function of test time elapsed. The error bars associated with analytical measurements represent twice the standard error of UDS means. 
Dewatering of the high-solids waste simulant slurry was carried out using only Filter 1. Figure 5.34 to Figure 5.37 show the operational conditions achieved on the PEP filtration systems during the HighSolids Scaling Test. Figure 5.34 shows estimates of the filter AV as a function of slurry UDS concentration. The velocity curves shown are based on the suction and discharge flowrates into and from the PEP pumping system. The difference between flow meter readings likely results from entrainment of air in the suction line to the pumping system. The target AV for the duration of the test was $15 \mathrm{ft} / \mathrm{s}$ with an allowable variation of up to $\pm 1.4 \mathrm{ft} / \mathrm{s}$. Significant deviation (up to $10 \%$ ) from this target occurred throughout the test as a result of increased pumping requirements. Specifically, dewatering of the slurry resulted in increased slurry rheology (both in terms of yield stress and consistency), which in turn required additional power from the pumps to achieve target flows and system pressure. As a result, the operating speeds for pumps T42A and T43A had to be adjusted periodically throughout the test. These adjustments appear as discontinuities in the filter AVs shown in Figure 5.34. The deviation from target AV was typically within acceptable tolerance; however, from $\sim 20 \mathrm{wt} \%$ to $\sim 22 \mathrm{wt} \%$ UDS, the suction flow to Pump T42A exceeded the allowable upper limit of $16.4 \mathrm{ft} / \mathrm{s}$.

Figure 5.35 shows the filter TMP for filter 1 as a function of slurry UDS concentration during dewatering operations. In contrast to AV, TMP is relatively well behaved (i.e., stable) throughout the test. The average TMP achieved for Filter 1 was 39.8 psid against a target TMP of $40 \pm 4$ psid. As in previous tests, the measured TMP is used to correct variations in filter flux derived from deviation of TMP from the target value of 40 psid.

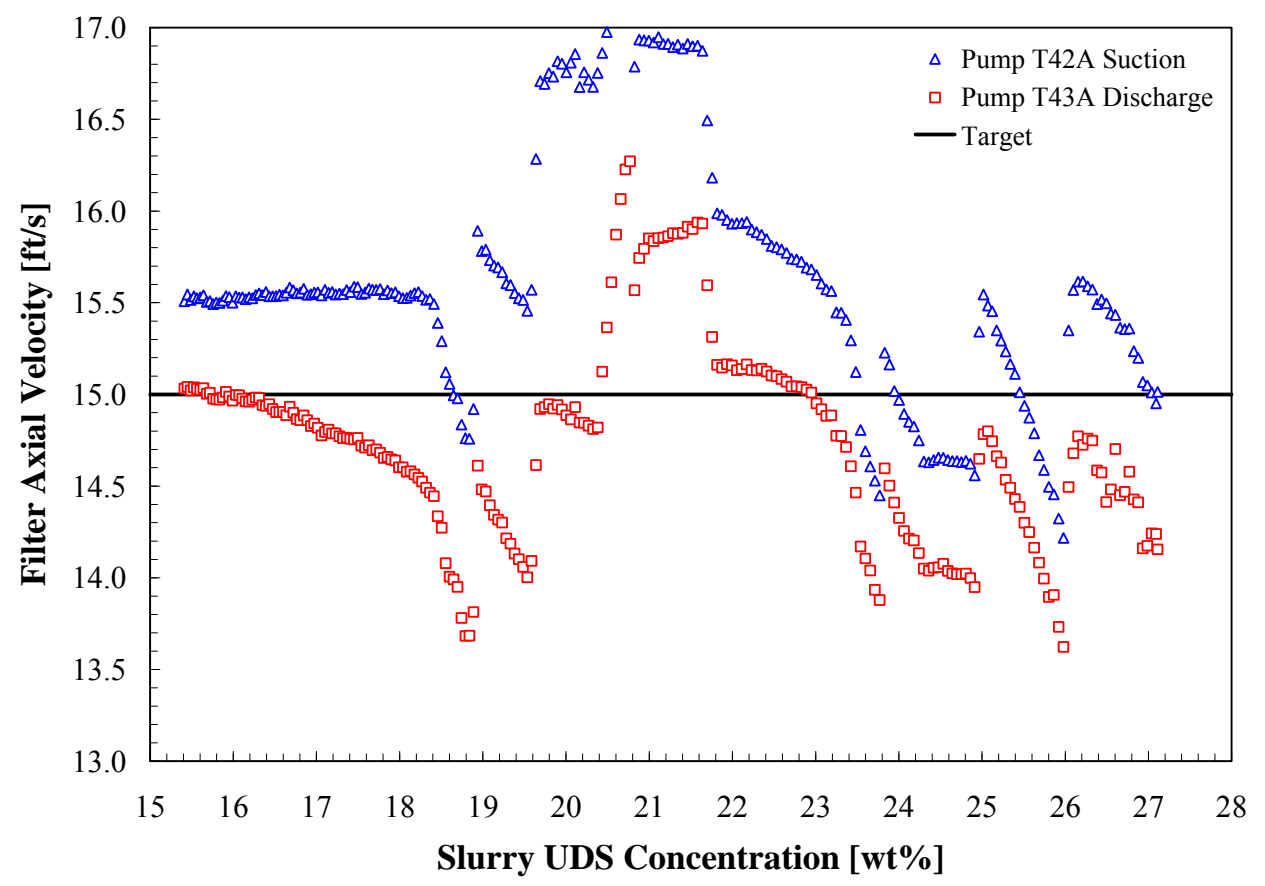

Figure 5.34. Filter AVs Achieved During the PEP High-Solids Scaling Test. The AVs for Pump T42A suction and Pump T43A discharge are based on sensors FT-0623 and FT-0635, respectively. The target velocity was $15.0 \pm 1.4 \mathrm{ft} / \mathrm{s}$. Control of AV was difficult because of changes to the slurry rheology. 


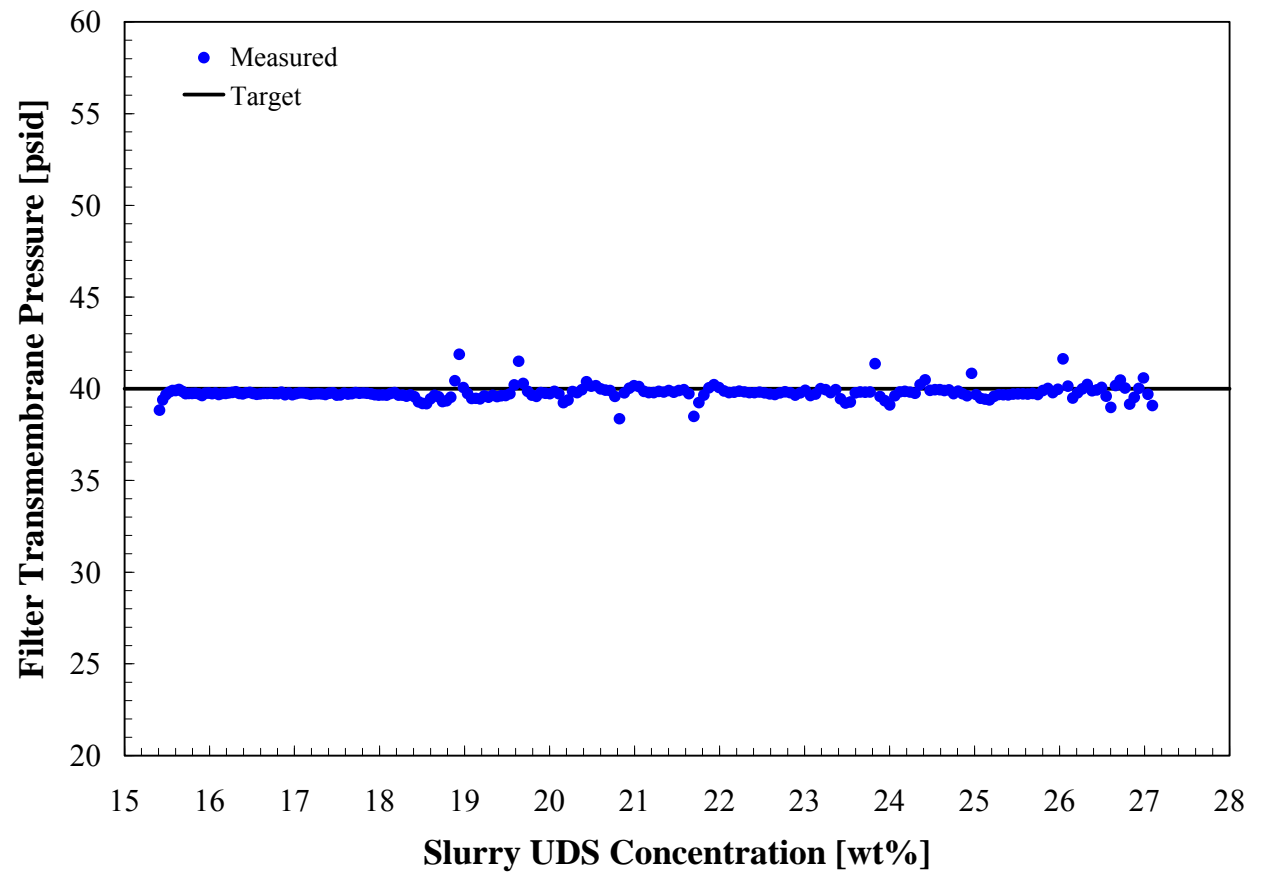

Figure 5.35. Filter TMP for Filter Bundle 1 During the PEP High-Solids Scaling Test. The target TMP was $40 \pm 4$ psid. The average TMP achieved for Filter 1 was $39.8 \pm 0.7$ psid $(\mu \pm 2 \sigma)$.

Figure 5.36 shows the APD across filter bundle 1 during PEP high-solids dewatering operations. From $15.4 \mathrm{wt} \%$ to $18.5 \mathrm{wt} \%$ UDS, the APD is relatively constant and approximately $13 \mathrm{psid}$. From 18.5 $\mathrm{wt} \%$ to $\sim 21 \mathrm{wt} \%$, the APD is discontinuous (as a result of changes in pump speed) but appears to evidence a slight upward curvature. At slurry UDS concentrations higher than $21 \mathrm{wt} \%$, the upward curvature is apparent. Such changes in APD curvature with increasing UDS concentration result from changes in the slurry rheology and possibly indicate a transition in the filter flow regime from turbulent flow to laminar flow.

The possibility of a turbulent-to-laminar flow transition is further supported by temperature sensors measurements for Tank T02A and filtration loop. These temperature measurements are summarized as functions of test UDS concentration in Figure 5.37. Here the following sensors are:

- TTK-0619-Tank T02A temperature

- TT-0791 -the filter bundle inlet temperature

- TT-0537 -the filter bundle outlet temperature

- TT-0513 -the outlet temperature for HX-T02A

- TT-0515 -the outlet temperature for HX-T03A.

Figure 5.37 shows that at $\sim 20$-wt $\%$ UDS, the filtration temperature sensors show a downward drift in temperature. According to the control strategy, the filtration operations should nominally maintain a filtration temperature of $25^{\circ} \mathrm{C}$. Increases in slurry rheological properties generally require additional pumping power to provide a given flowrate and pressure, which in turn would tend to heat the slurry 
through viscous dissipation of energy and friction. Thus, the downward drift in temperature is contrary to what would be expected during dewatering. However, because the thermowells associated with these filtration loop temperature sensors were installed such that they did not extend into the process flow (as they should have for proper temperature measurement), the temperature sensors were isolated from the flowing part of the fluid during the high-solids scaling test. It is speculated that the decline in filter-loop temperature readings results from stagnation of slurry in the thermowell ports. ${ }^{(a)}$ The slurry filling the thermowells stagnates at $\sim 20 \mathrm{wt} \%$ and slowly cools to ambient temperature through conductive and radiant heat transfer. It is likely that for the simulant slurry employed in the current tests that fluid stagnation only affects high-solids concentrations slurries (i.e., $>20-\mathrm{wt} \%$ UDS). For a more dilute slurry (such as the $6.9-\mathrm{wt} \%$ slurry used in low solids scaling test), the temperature sensors installed in the filtration loop may provide a better representation of filtration temperature; however, the NCR 42402.1 prevents using these temperature sensors for quality-affecting work.

Process temperature during the High-Solids Scaling Test was controlled using TT-0513 located at the outlet of the cooling heat exchanger (HX-T02A). Fluid stagnation at this temperature sensor caused the automatic temperature control systems on PEP to shut chiller HX-T02A off when the apparent slurry temperature reached $23^{\circ} \mathrm{C}$. Once the HX-T02A shut off, the heat input from pumps T42A and T43A was no longer being withdrawn from the system. As a result, significant temperature variation was observed in well-mixed parts of the system as indicated by the sudden rise in Tank T02A temperature from $\sim 23^{\circ} \mathrm{C}$ to $\sim 27^{\circ} \mathrm{C}$ over $23 \mathrm{wt} \%$ to $24 \mathrm{wt} \%$ UDS. Manual control of the PEP temperature control systems resulted in a variable temperature near the end of the test. Corresponding variations appear in the filtration loop temperature sensors but significantly damped.

Currently, the information shown in Figure 5.36 and Figure 5.37 provides the best indication that the mechanisms governing temperature drift are caused by a turbulent-to-laminar transition and accompanying fluid stagnation. The assumption here is that loss of flow turbulence in the filter-loop leads to a loss of fluid mixing (and fluid stagnation) in the thermowell. Applying standard transitional flow theories used to predict critical flow velocities for turbulent flow in non-Newtonian slurries is hindered in the current case by 1) complex flow geometries at the entrance and inside the filter bundles and 2) cake formation resulting from radial permeate flow through the porous filter membranes. Complex flow geometries include bends at the entrance and exit to each bundle and contraction and expansion of slurry flow as it enters and exits the parallel filter element array inside each bundle. As a result, flows may be non-steady, and slurry solids may be non-uniformly (i.e., non-symmetrically) distributed. Cake formation results from the transport of slurry solids to the filter surface (via filtration) and usually becomes more severe as the slurry is dewatered. As higher UDS concentrations are approached, axial shear caused by cross flow may not be sufficient to fully overcome the adhesive and/or frictional forces that fix the filter cake to the surface of the element. Because existing theories for transitional flow do not account for the impacts of such cake formation, they may not accurately predict transition points in flows where a filter cake coats the boundaries of the flow path. If these complexities are neglected, then standard theories for predicting flow transition in non-Newtonian slurry predict that the slurry will transition from turbulent-to-laminar flow somewhere between 12 to $27 \mathrm{wt} \%$ UDS (depending on location).

(a) An evaluation documented in NCR 42402.1 indicates that data from TT-0537, TT-0513, and TT-0515 should not be used for quality-affecting work, but may be used for qualitative purposes, and that TT-0791 should not be used at all because of this issue. 


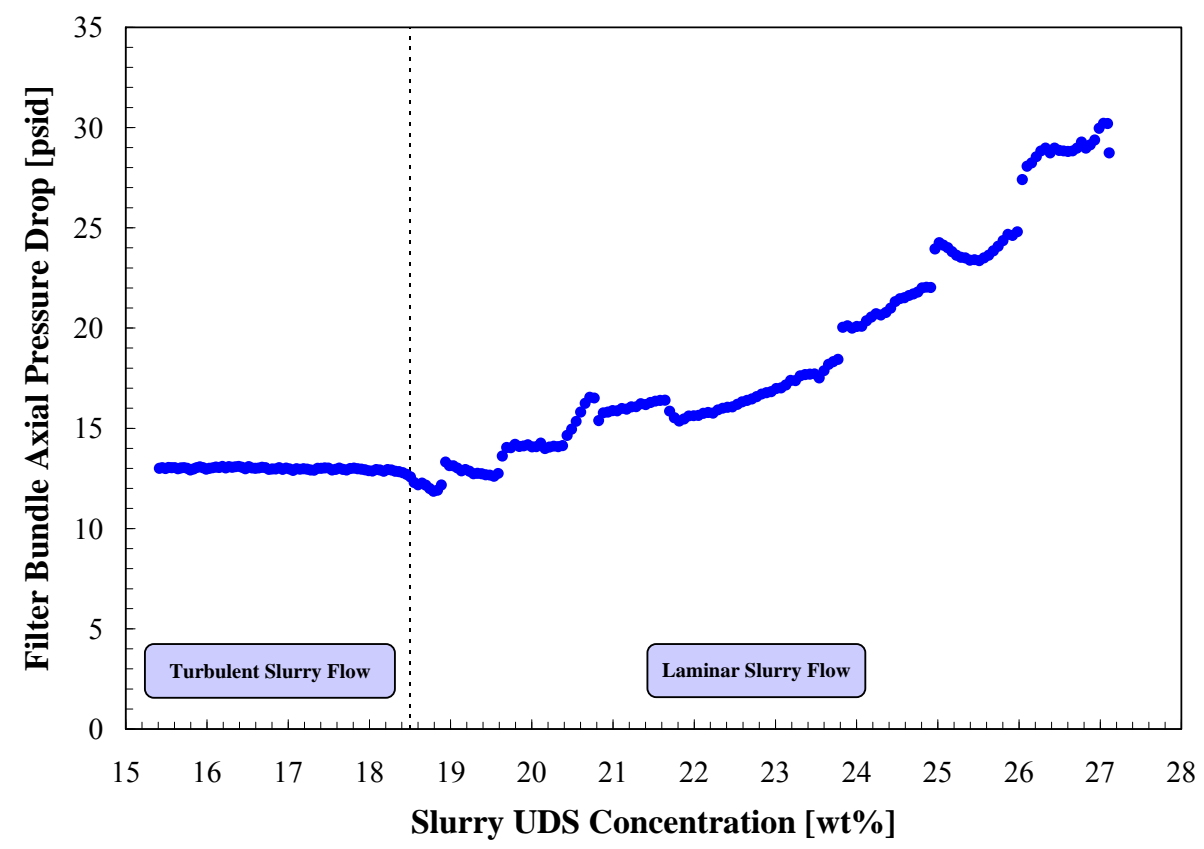

Figure 5.36. Filter APD Across Filter Bundle 1 During the PEP High-Solids Scaling Test. The upper allowable limit for APD was 50 psid for all filter. The appearance of upward curvature in the APD versus slurry UDS near $18.5 \mathrm{wt} \%$ is suggestive of a transitional region where flow transitions from turbulent to laminar flow. Estimates indicate that the laminar-toturbulent transition will occur over 12 to $27 \mathrm{wt} \%$ (depending on location).

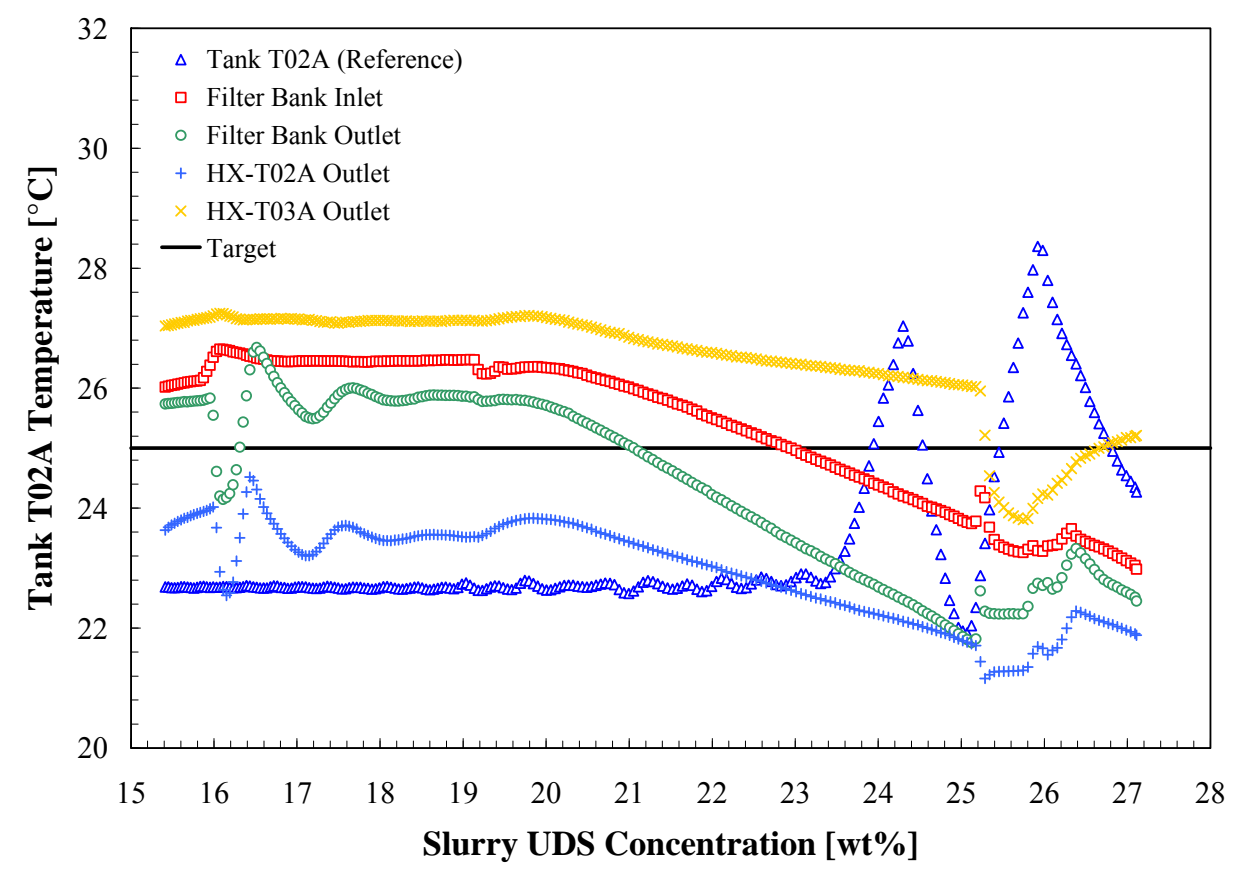

Figure 5.37. Temperatures Measured in Vessel UFP-VSL-T02A and in the Filtration Loop During the PEP High-Solids Scaling Test. All sensors but that for Tank T02A should be considered "for information only." The target temperature of the filtration loop during testing was $25 \pm 2{ }^{\circ} \mathrm{C}$. 
Because of the potential for fluid stagnation in the filtration loop thermowells, fluid temperature corrections used throughout this report have been based on temperature sensors corresponding to wellmixed portions of the system, specifically Tank T02A. Likewise, temperature corrections for filter flux measured during the High-Solids Scaling Test are based on deviations in Tank T02A temperature from the test target of $25^{\circ} \mathrm{C}$. While this temperature may not be entirely representative of the temperature that exists in the filter loop, it provides a consistent basis for flux temperature corrections. As shown by Figure 5.37, the difference between Tank T02A temperatures and that in the filtration loop can be up to $\sim 5^{\circ} \mathrm{C}$ (for thick slurries). The impact on the scaling analysis is on the order of the magnitude of flux correction ( 10\%); however, it should be noted that both CUF and PEP filter fluxes are corrected to their respective slurry reservoir temperatures. However, as discussed in Section 4.4, CUF and PEP differ in the location of their heat exchange systems. For CUF, the heat exchanger is located immediately after the pump system and can remove mechanical heat from the slurry before filtration. In contrast, the PEP heat exchanger (UFP-HX-T02A) is located immediately after the filter system. As such, PEP permeate rates are likely affected by differences in filtration loop and tank temperature to a greater degree than those for CUF.

Finally, Figure 5.38 shows the dewatering curve measured during the High-Solids Scaling Test. The dewatering curve shows a "knee" at $21.7 \mathrm{wt} \%$, indicating the approach to a gel-limited filtration regime. As shown, the portion of the dewatering curve beyond $21.7 \mathrm{wt} \%$ is expected to be nominally linear (in accordance with the theory outlined in Section 3.4.6). For the PEP dewatering curve, the curve beyond $21.7 \mathrm{wt} \%$ is generally linear, but shows slight deviations near $21.7 \mathrm{wt} \%$ and from $25 \mathrm{wt} \%$ to $27 \mathrm{wt} \%$. These deviations are associated with variations in both filter AV and slurry temperatures not fully accounted for by the flux correction equations.

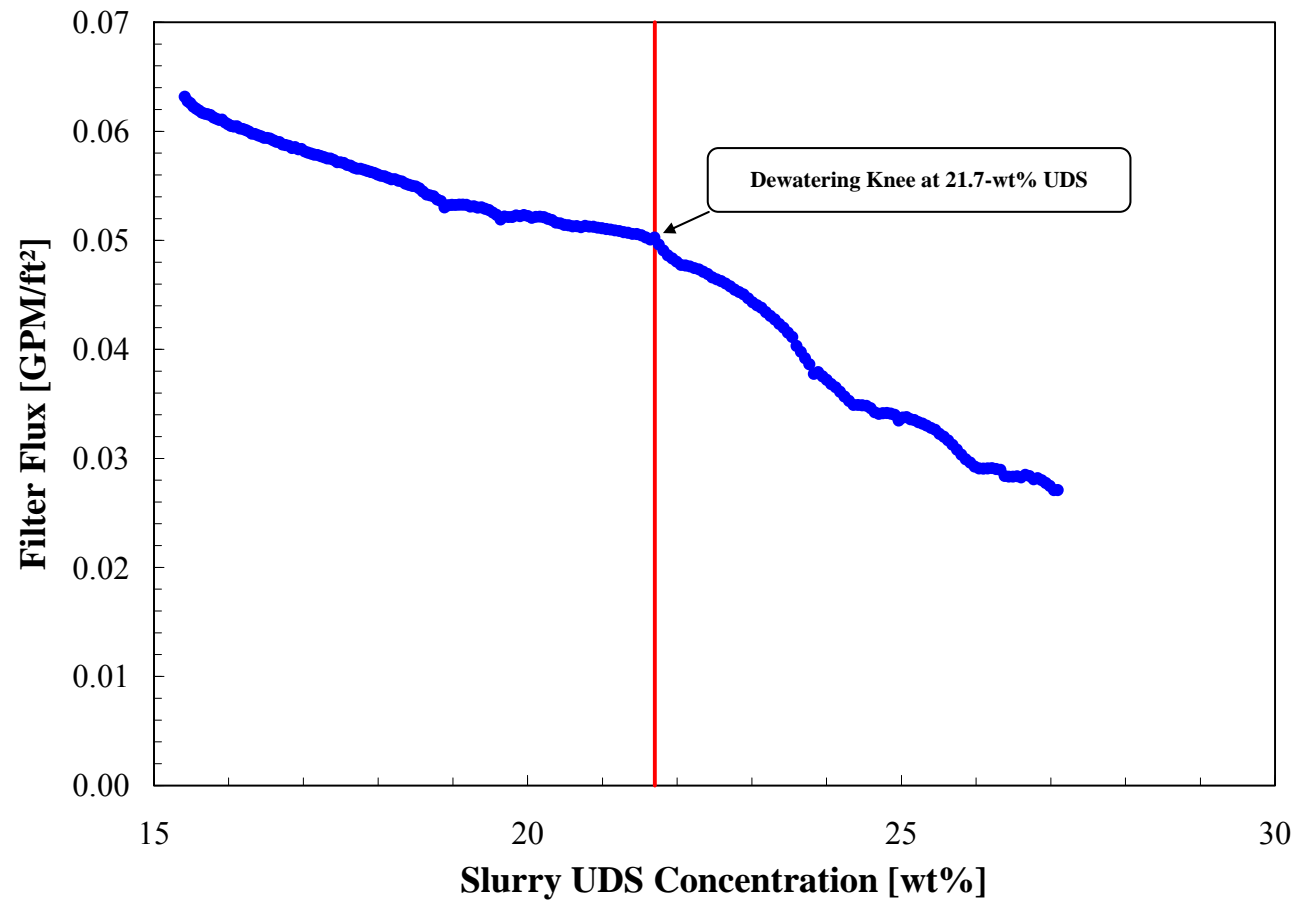

Figure 5.38. Dewatering Curve for the High-Solids Simulant Measured During the PEP High-Solids Scaling Test. A dewatering "knee" was observed at 21.7 wt\% UDS. 


\subsubsection{CUF Operations}

The waste simulant slurry for parallel CUF testing was delivered from PEP (the sample was obtained before the start of the high solids filter test) and tested over March 25-27, 2009. Two separate high-solids dewatering operations were performed - an initial dewatering and a repeat dewatering. The operational parameters of each test are summarized in Table 5.11. The initial test employed $25.0 \mathrm{~kg}$ of an asmeasured $15.3 \mathrm{wt} \%$ UDS slurry for a slurry solids-to-filter surface area ratio of $14.6 \mathrm{~kg} / \mathrm{ft}^{2}$. Likewise, the repeat test employed $24.7 \mathrm{~kg}$ of an as-measured $15.3 \mathrm{wt} \%$ UDS slurry for a slurry solids-to-filter surface area ratio of $14.4 \mathrm{~kg} / \mathrm{ft}^{2}$. Both CUF high-solids tests used a slurry solids-to-filter surface area ratio that is similar to, but slightly higher than, the corresponding PEP ratio of $13.9 \mathrm{~kg} / \mathrm{ft}^{2}$. All ratios fall in the range anticipated for use in WTP (1.7 to $16 \mathrm{~kg} / \mathrm{ft}^{2}$ - see Section 5.1.1).

Table 5.11. Operational Parameters for the High-Solids Dewatering Tests

\begin{tabular}{|c|c|c|}
\hline Parameter & $15 \mathrm{fps}$ & 15 fps repeat \\
\hline $\begin{array}{l}\text { Total mass of slurry in CUF } \\
\text { system (includes BP hold-up): }\end{array}$ & $25.0 \mathrm{~kg}$ & $24.7 \mathrm{~kg}$ \\
\hline $\begin{array}{l}\text { Slurry solids-to-filter surface area } \\
\text { ratio: }\end{array}$ & $14.6 \mathrm{~kg} / \mathrm{ft}^{2}$ & $14.4 \mathrm{~kg} / \mathrm{ft}^{2}$ \\
\hline Starting wt\% UDS, measured: & $15.3 \pm 4.0 \%$ & $15.3 \pm 4.0 \%$ \\
\hline Ending wt $\%$ UDS, calculated: & $29.5 \%$ & $32.4 \%$ \\
\hline Ending wt\% UDS, measured: & $32.5 \pm 11.1 \%$ & $38.8 \%$ \\
\hline Start of dewatering & 3/25/2009 13:20 PST & 3/27/2009 09:11 PST \\
\hline End of dewatering & 3/25/2009 17:03 PST & 3/27/2009 14:35 PST \\
\hline Total test time elapsed: & 3.72 hours & 5.40 hours \\
\hline Mixer impeller configuration & \multicolumn{2}{|c|}{$\begin{array}{l}\text { Two impellers: a) 2-inch diameter propeller at the end of the shaft at } \\
\text { one tank radius from the bottom b) } 3 \text {-inch diameter, pitched, } 3 \text {-blade } \\
\text { turbine positioned } 5 \text { inches above the propeller Both impellers were } \\
\text { submerged in the slurry for the majority of the dewatering test. When } \\
\text { the tank level approached the upper turbine, the stir shaft was raised } \\
\text { such that the upper turbine was above the liquid. This was to prevent } \\
\text { splattering in the tank. }\end{array}$} \\
\hline Mixer speed & \multicolumn{2}{|c|}{$\begin{array}{l}\text { Typically } 1140 \mathrm{rpm} \text {, but decreased as needed (down to } 700-800 \mathrm{rpm} \text { ) } \\
\text { to prevent slurry vortex and air entrainment }\end{array}$} \\
\hline
\end{tabular}

From the initial slurry concentration and mass provided, the evolution of slurry UDS during both tests can be determined through a mass balance of slurry and collected permeate. Figure 5.39 shows the result of this analysis. It indicates that the slurry was dewatered from $15.3 \mathrm{wt} \%$ to $29.5 \mathrm{wt} \%$ UDS during for the initial test. Mass balance analysis of the repeat test indicates a dewatering of the $15.3-\mathrm{wt} \%$ slurry to a final concentration of $32.4 \mathrm{wt} \%$.

For both tests, a filter AV and TMP of $15 \mathrm{ft} / \mathrm{s}$ and $40 \mathrm{psid}$, respectively, were targeted. The actual filter velocity and TMP achieved during testing (as a function of slurry UDS concentration) are shown in Figure 5.40 and Figure 5.41. Overall, AV and TMP were stable throughout both tests. Small deviations were corrected through periodic adjustment of the cold-CUF filtration system pump speed and backpressure valve. An average AV of $15.0 \pm 0.1 \mathrm{ft} / \mathrm{s}(\mu \pm 2 \sigma)$ was achieved for both tests. Likewise, an average TMP of $41 \pm 2$ psid was achieved for both tests. The measured TMP were used to correct permeate flux for deviations from the target TMP of 40 psid. 
Figure 5.42 shows the APD for the cold-CUF filter system during both tests. Because of process noise at the filter inlet pressure sensor, it is difficult to evaluate the APD trends at concentrations below $25 \mathrm{wt} \%$ UDS. As such, the existence of a transitional flow regime in the CUF filter at UDS concentrations similar to that observed in PEP cannot be assessed from the current data. More accurate pressure measurements are required to determine if there is a transitional region in CUF data from 18 wt $\%$ to $25 \mathrm{wt} \%$. Beyond $25 \mathrm{wt} \%$, there is a visible upward trend in APD with UDS. At $29 \mathrm{wt} \%$ UDS, both tests show a marked increase in APD. Care must be taken when comparing the CUF APD behavior in Figure 5.42 to the PEP APD behavior in Figure 5.36. On first inspection, one might conclude that pressure increase in CUF starts at much higher concentrations than in PEP. However, the upper concentration bound tested in CUF far exceeds that tested in PEP. At these higher concentrations, pressure effects derived from changes in rheology are expected to be much more severe and will dwarf any moderate changes in pressure observed over $21 \mathrm{wt} \%$ to $27 \mathrm{wt} \%$ (which are already difficult to observe in CUF because of a lack of pressure sensor sensitivity).

The APD trends with increasing slurry UDS shown in Figure 5.36 and Figure 5.42 are indicative of increased slurry yield stress and consistency (and associated flow pressure drops) during dewatering in laminar flow regions. Based on Figure 5.42, it appears that a turbulent-to-laminar transition does impact the cold-CUF test. As stated previously, it is difficult to determine the point at which this transition occurs using the current CUF pressure sensor. However, examination of Figure 5.42 hints at an initial rise in APD around $23 \mathrm{wt} \%$, which would correspond approximately to the region of first rise in the PEP APD. As such, we can tentatively propose that flow dynamics in the filters are similar for both CUF and PEP during dewatering operations. This means that any flow transitions (and potentially solids deposition that results in laminar flow) will affect both CUF and PEP filter flux performance.

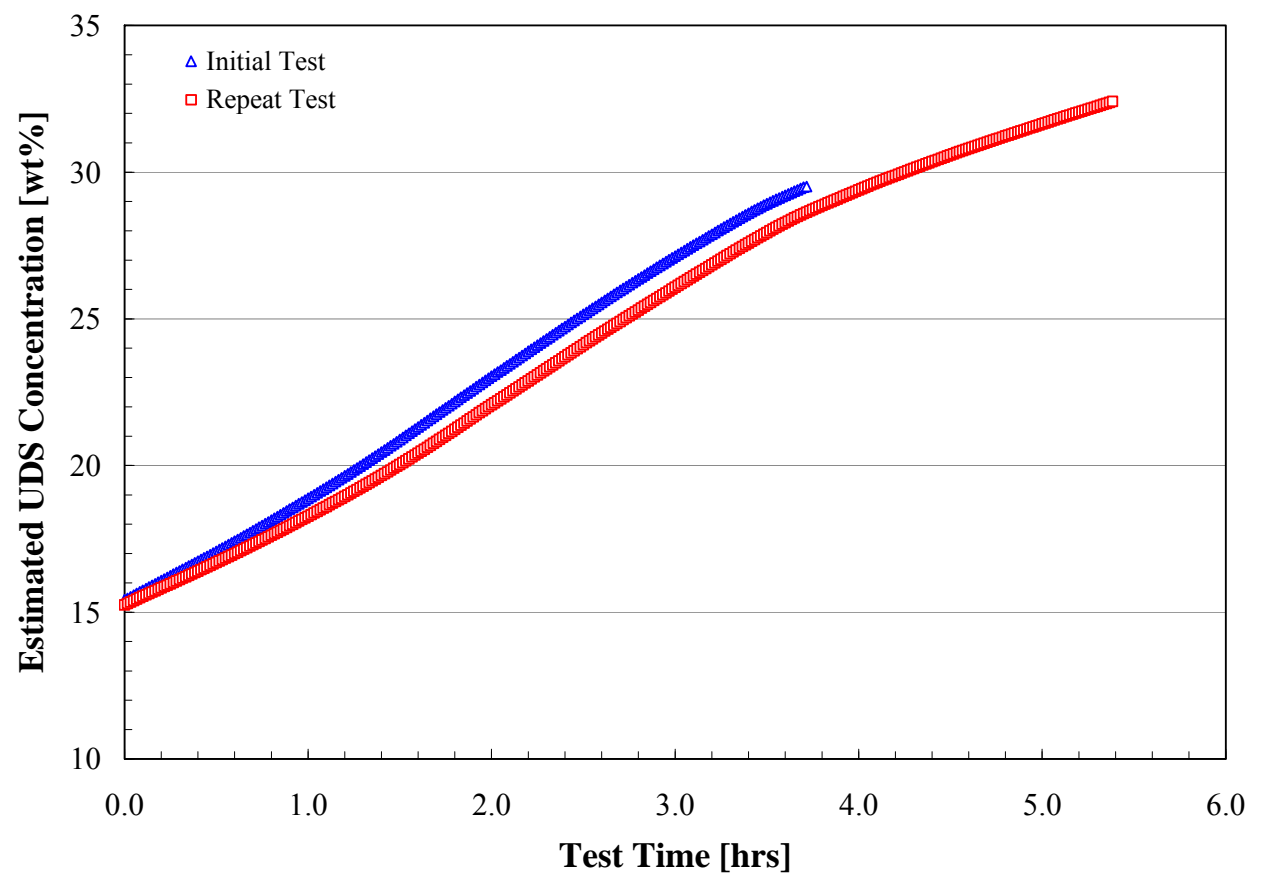

Figure 5.39. Slurry UDS Concentrations Tested During the Initial and Repeat and CUF High-Solids Scaling Test. Both UDS curves are estimated using slurry mass balance calculations. 


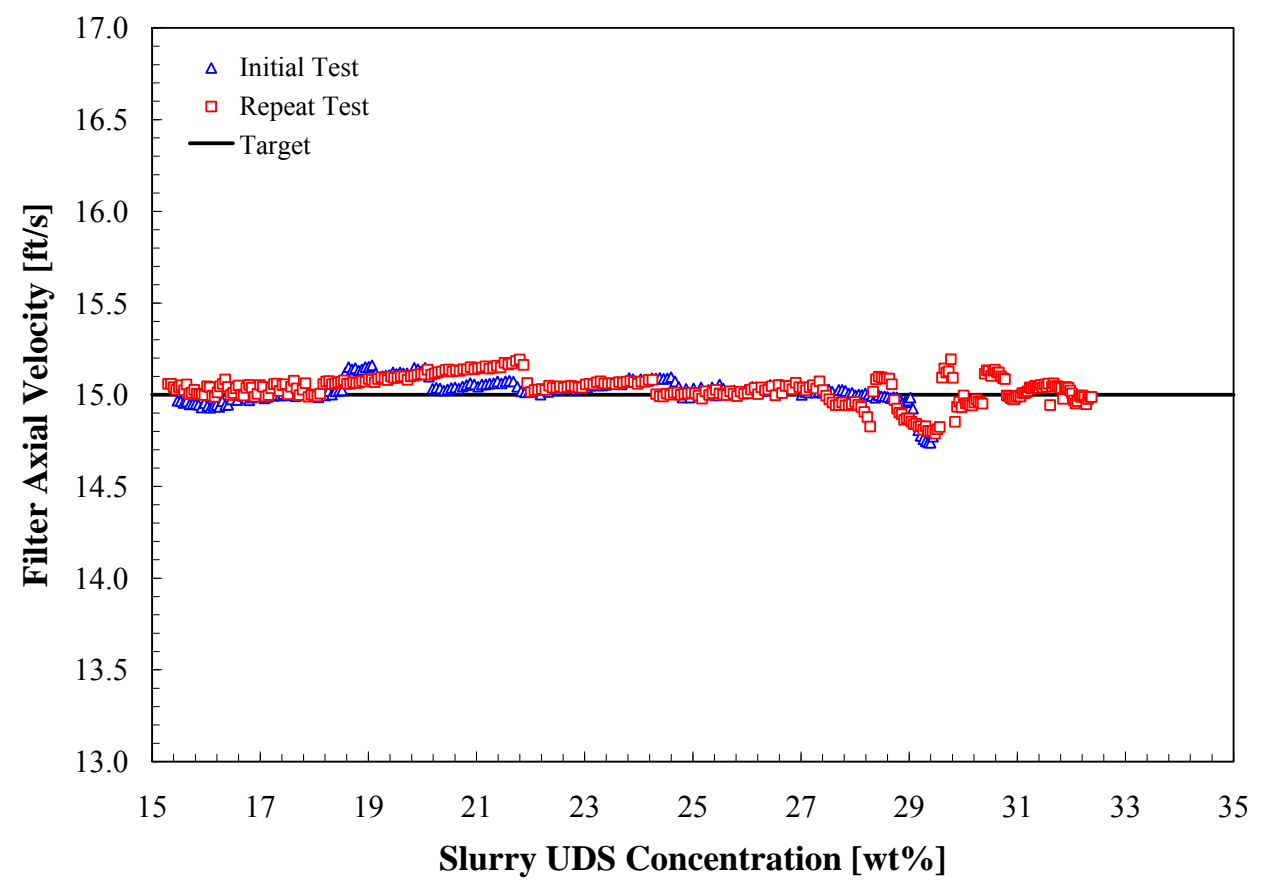

Figure 5.40. Filter AVs Achieved During the Initial and Repeat CUF High-Solids Scaling Test. For both tests, an average AV of $15.0 \mathrm{ft} / \mathrm{s}$ was achieved.

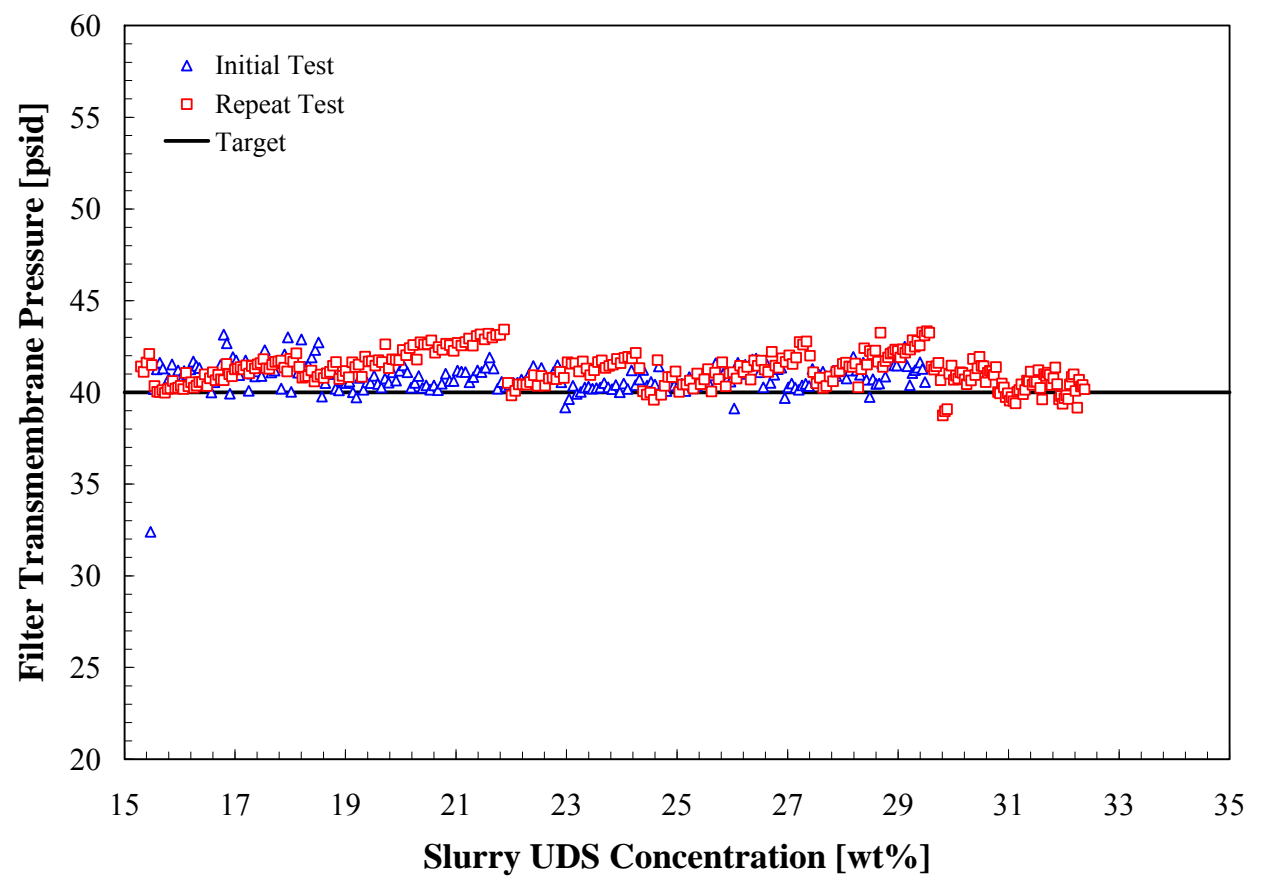

Figure 5.41. Filter TMP Achieved During the Initial and repeat CUF High-Solids Scaling Test. The target TMP was 40 psid. For both tests, an average TMP of 41 psid was achieved. 


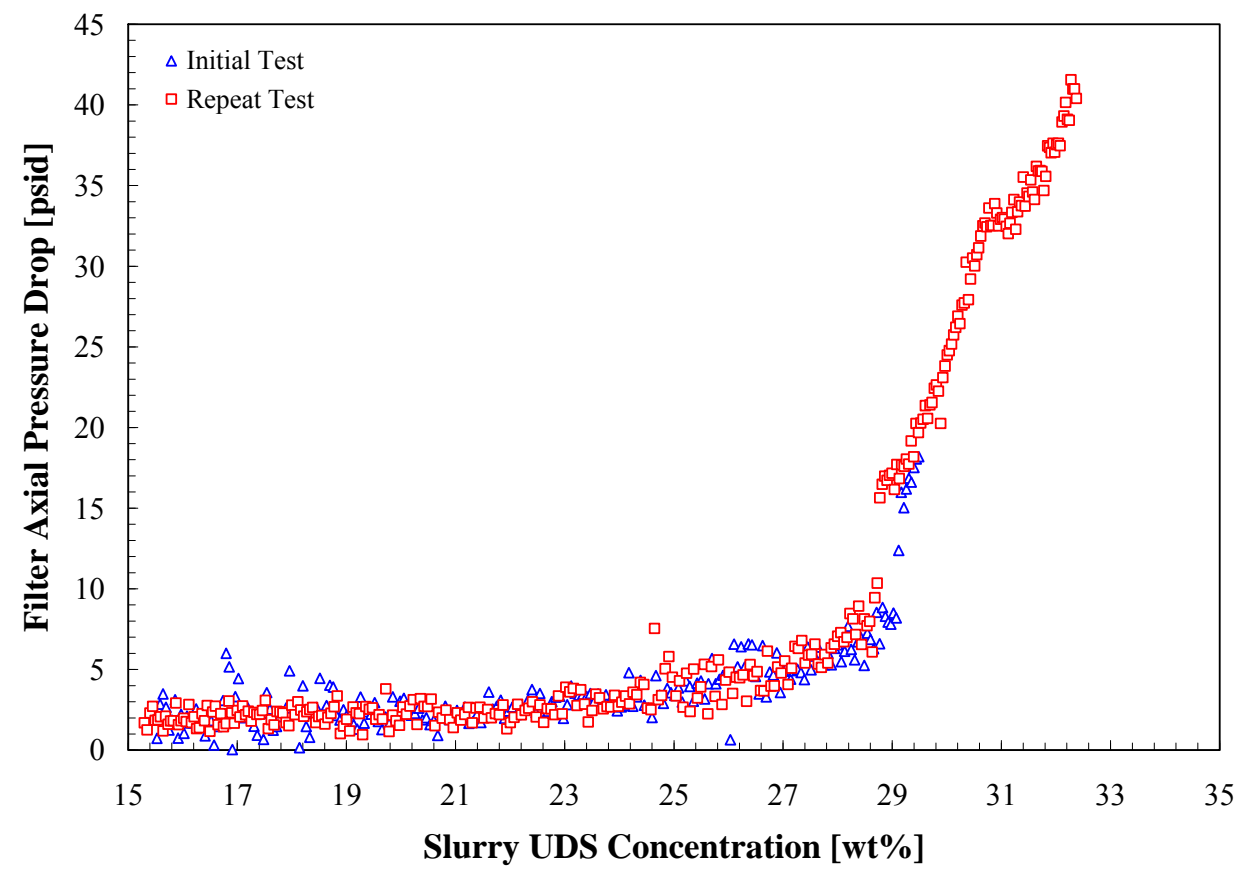

Figure 5.42. Filter APD Across the CUF Filter During the Initial and Repeat CUF High-Solids Scaling Test. The appearance of upward curvature in the APD versus slurry UDS is difficult to assess because of the low signal-to-noise ratio in the pressure transducer readings at low UDS. Flow may be transitional as early as $21 \mathrm{wt} \%$ UDS and is fully laminar at $\sim 29 \mathrm{wt} \%$ UDS.

Figure 5.43 shows the evolution in slurry reservoir temperature during the initial and repeat CUF high-solids dewatering tests. In both cases, the increase in slurry rheology (i.e., yield stress and consistency) and the corresponding increase in pumping power required to circulate the slurry exceed the cold-CUF system's cooling capacity at $\sim 25 \mathrm{wt} \%$ UDS. Beyond this UDS, the temperature appears to deviate above the control temperature of $25^{\circ} \mathrm{C}$. The slurry reservoir temperature measurements shown in Figure 5.43 were used to correct the measured permeate flux for deviations in test temperature from the target of $25^{\circ} \mathrm{C}$. As discussed in Section 4.4, the filtration temperature in CUF is expected to closely match the temperature in the CUF slurry reservoir because of the heat exchanger location. This contrasts with PEP operations, where the filtration temperature is expected to be several degrees higher than Tank T02A because of mechanical heating by Pumps T42A and T43A. This is a result of the CUF heat exchange system being installed before the filter area, allowing removal of mechanical pumping heat from the slurry supernate before filtration. 


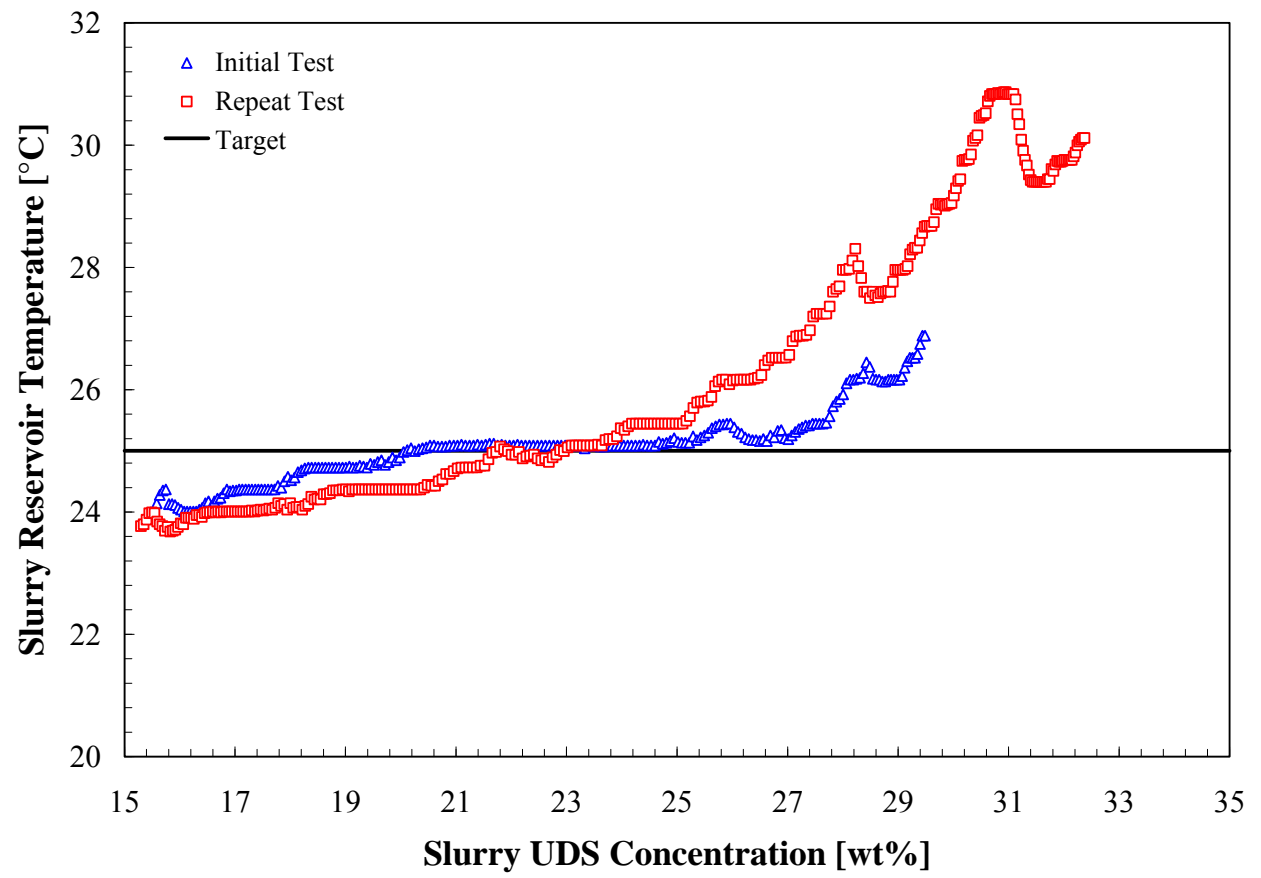

Figure 5.43. Temperature of the CUF Slurry Reservoir During the Initial and Repeat CUF High-Solids Scaling Test. The target temperature of the filtration loop during testing was $25^{\circ} \mathrm{C}$. The chiller unit attached to the CUF did not have sufficient capacity to remove heat generated by the pumping of the viscous slurry at UDS concentrations greater than $27 \mathrm{wt} \%$.

Figure 5.44 shows the dewatering curves measured for the initial and repeat CUF High-Solids Scaling Test. Both CUF curves show a dewatering "knee" at $21.2 \mathrm{wt} \%$ that indicates a transition to filtration dynamics governed by the limiting gel concentration. This concentration is similar to the $21.7 \mathrm{wt} \%$ dewatering knee observed for PEP dewatering. At UDS higher than the knee concentration, both CUF dewatering curves are log-linear up to point where the slurry thickness appears to exceed the operating (i.e., pumping/cooling) capacity of the cold-CUF system (near $\sim 27 \mathrm{wt} \%$ UDS). It is speculated that the non-linearity in the dewatering curves at concentrations higher than $27 \mathrm{wt} \%$ result from temperature deviations and inability to meet the increased pumping requirements dictated by the high APD. 


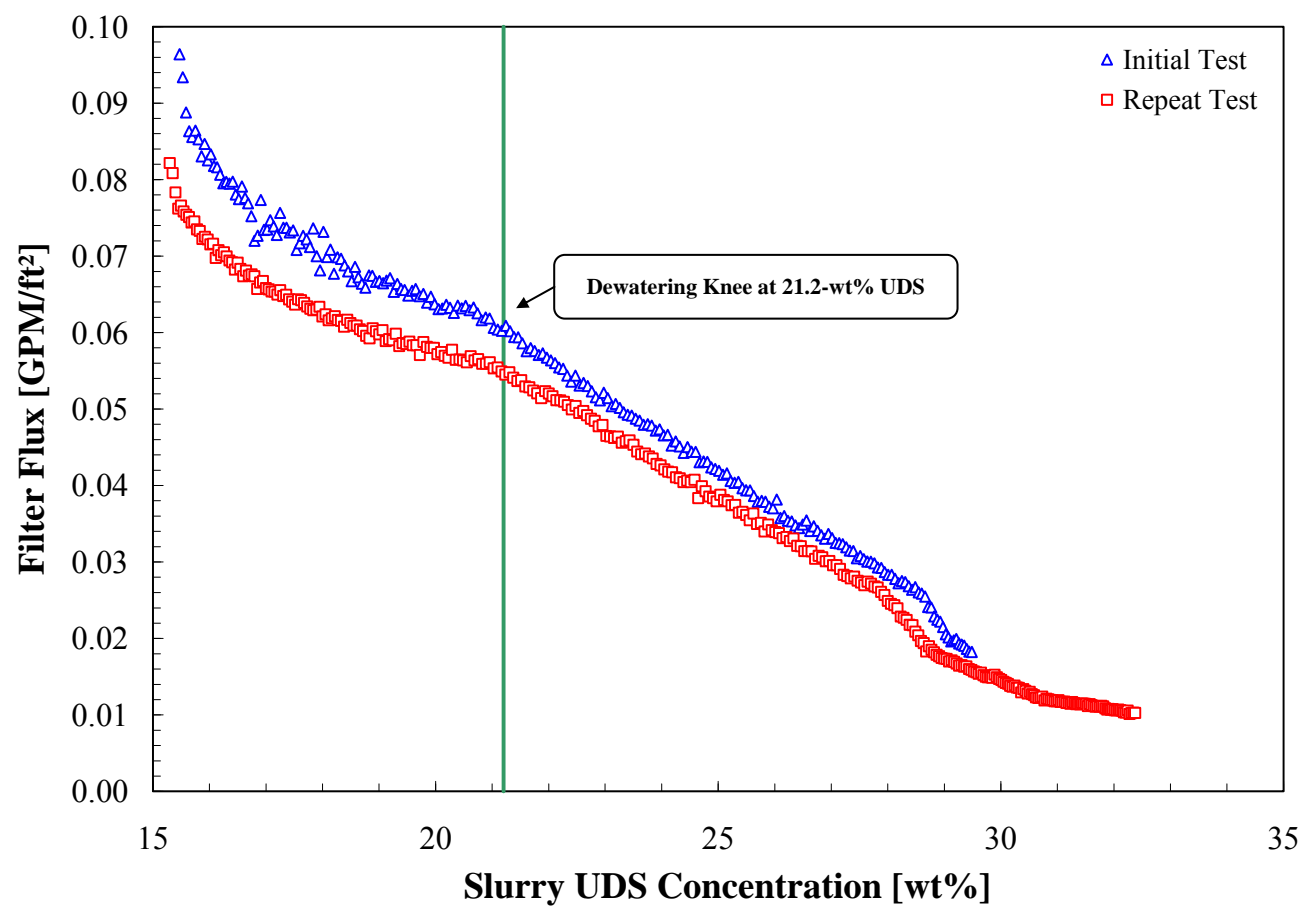

Figure 5.44. Dewatering Curves for the High-Solids Simulant Measured During the Initial and Repeat CUF High-Solids Scaling Tests. For both curves, a dewatering "knee" was observed at $21.2 \mathrm{wt} \%$ UDS.

\subsubsection{Analysis of Filter Scaling for the High-Solids Test}

The dewatering curves for PEP and CUF form the basis for the high-solids scaling analysis.

Figure 5.45 shows a comparison of the filter flux measured as a function of slurry UDS concentration for PEP and CUF high-solids tests. To assess the scaling factor for high-solids dewatering operations, these dewatering curves were characterized using the general filter flux equation for slurry operations approaching the gel concentration:

$$
J=k \cdot \ln \left[\frac{C_{s}}{C_{g}}\right]
$$

Specifically, the characteristic mass transfer coefficient $(k)$ and limiting gel concentration $\left(C_{g}\right)$ were determined for each dewatering experiment using linear regression analysis. The results of this analysis are shown in Figure 5.46, Figure 5.47, and Figure 5.48. These figures correspond to the PEP dewatering, initial CUF dewatering, and repeat CUF dewatering operations, respectively. 


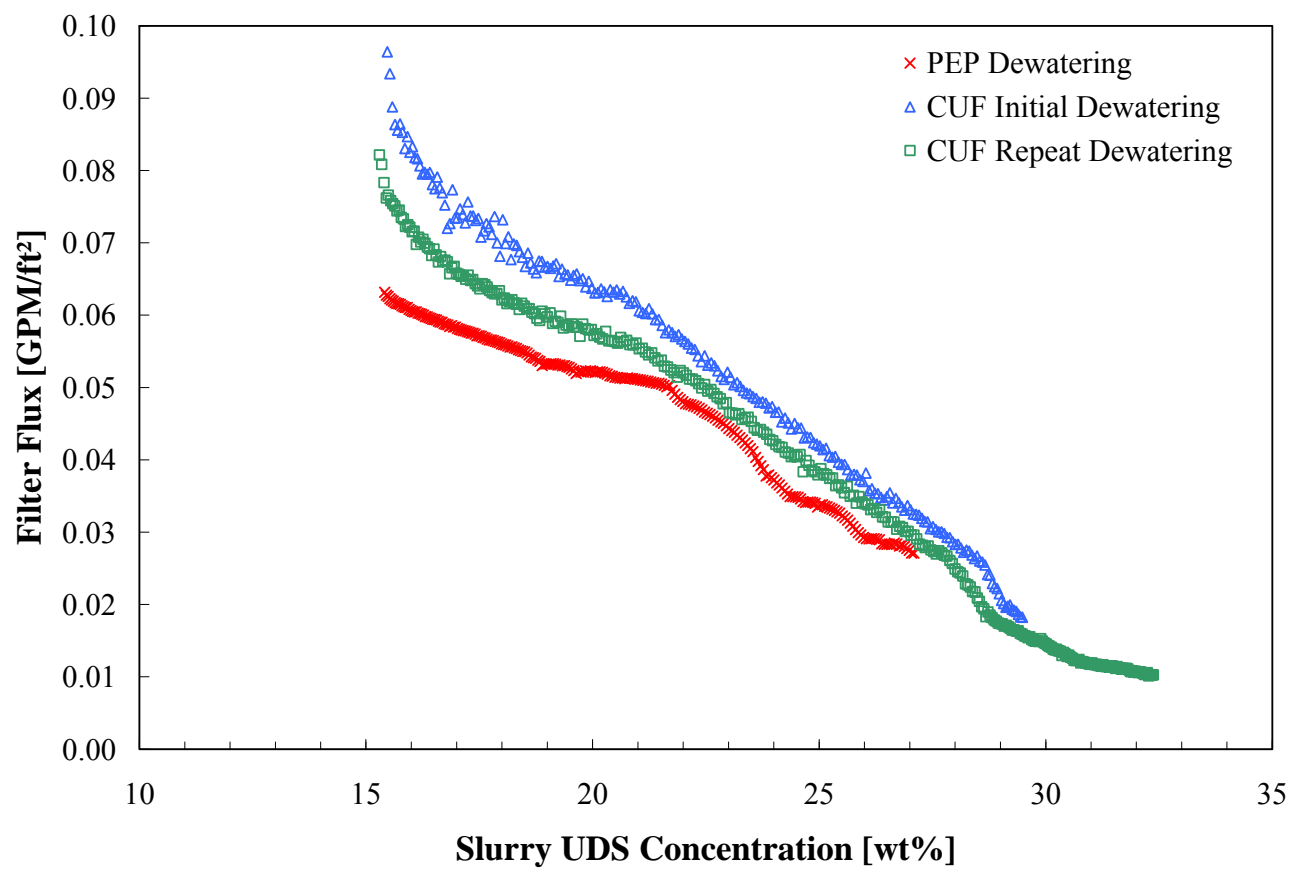

Figure 5.45. Comparison of the PEP and CUF Dewatering Curves

The PEP dewatering curve analysis shown in Figure 5.46 only considers UDS concentrations from $21.7 \mathrm{wt} \%$ to $27.1 \mathrm{wt} \%$. These data correspond to the gel limited dewater region (i.e., past the dewatering knee at $21.7 \mathrm{wt} \%$ ). For analysis of dewatering data, regions associated with unstable AV/TMP conditions are typically excluded. However, most of the PEP dewatering data beyond the dewatering knee were impacted by unstable operations. Exclusion of this data would lead to a highly limited set of data for regression. The impact of including data from the region of unstable PEP operations (i.e., those associated with possible fluid stagnation in the filtration loop) is a higher associated uncertainty in the PEP dewatering parameters (relative to the CUF dewatering parameters).

In contrast, linear regression analysis of the both CUF dewatering curves only considers concentrations from $21.2 \mathrm{wt} \%$ to $28.4 \mathrm{wt} \%$. This region incorporates CUF filter flux data beyond the dewatering knee (at $21.2 \mathrm{wt} \%$ ) while avoiding the unstable periods of operation associated with high slurry UDS. 


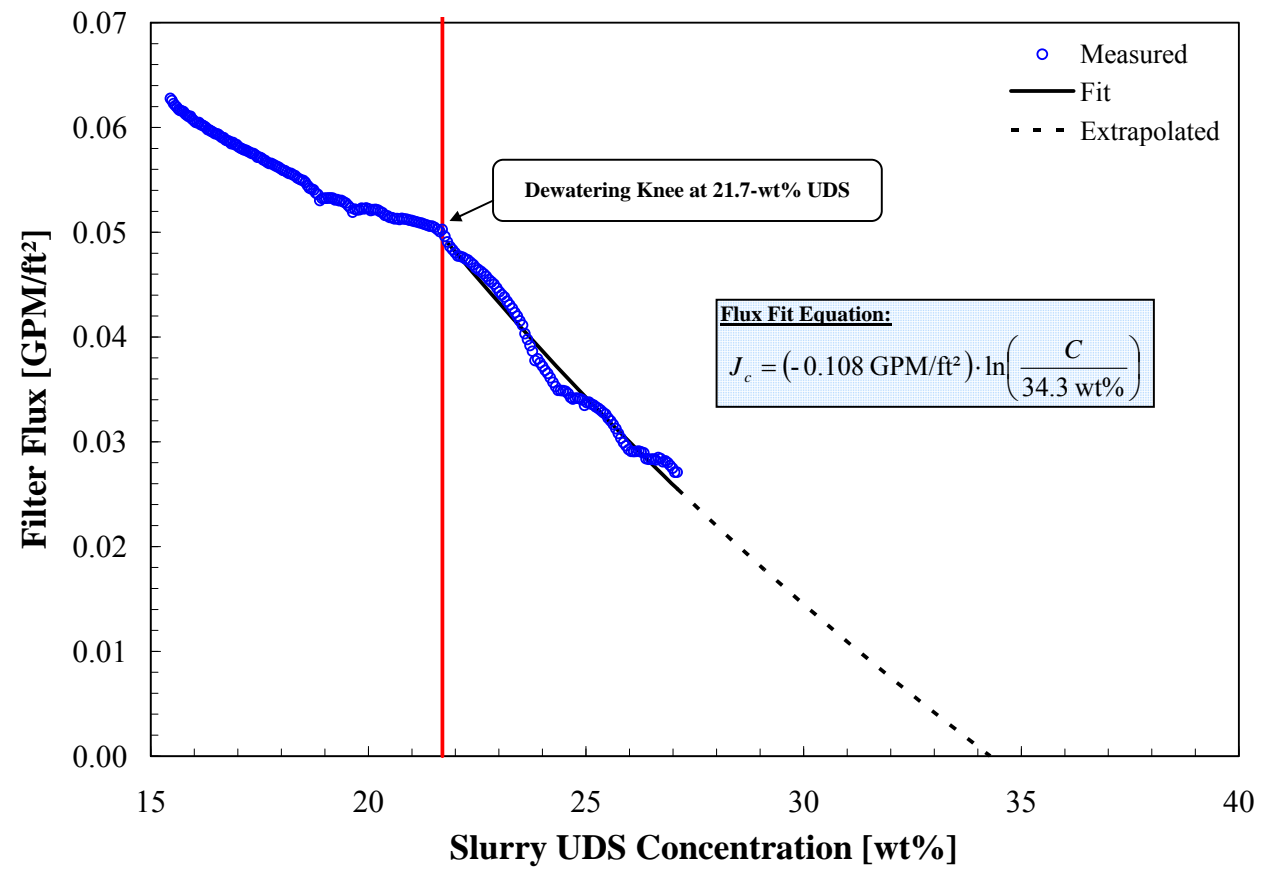

Figure 5.46. Analysis of the PEP Dewatering Curve Measured During the PEP High-Solids Scaling Test

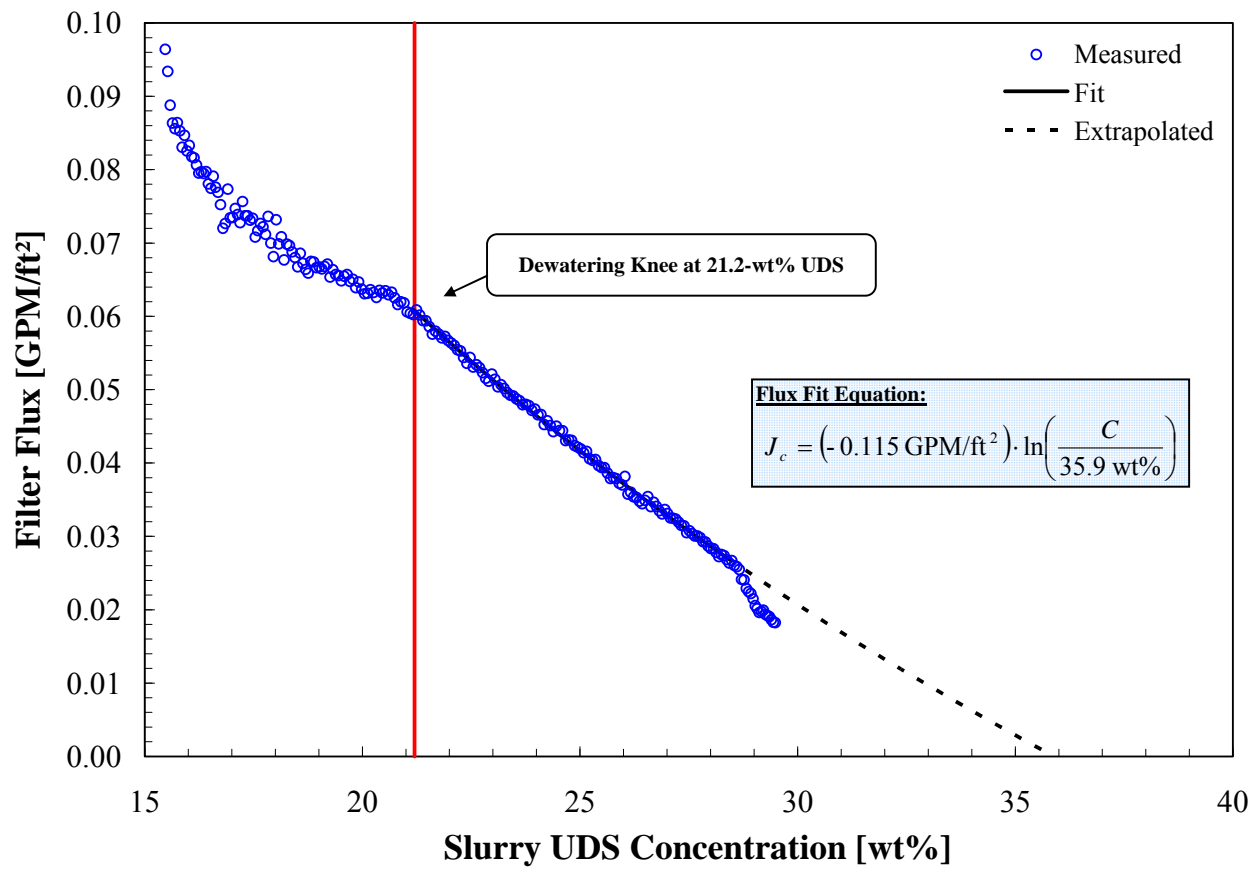

Figure 5.47. Analysis of the Initial Dewatering Curve Measured During the CUF High-Solids Scaling Test 


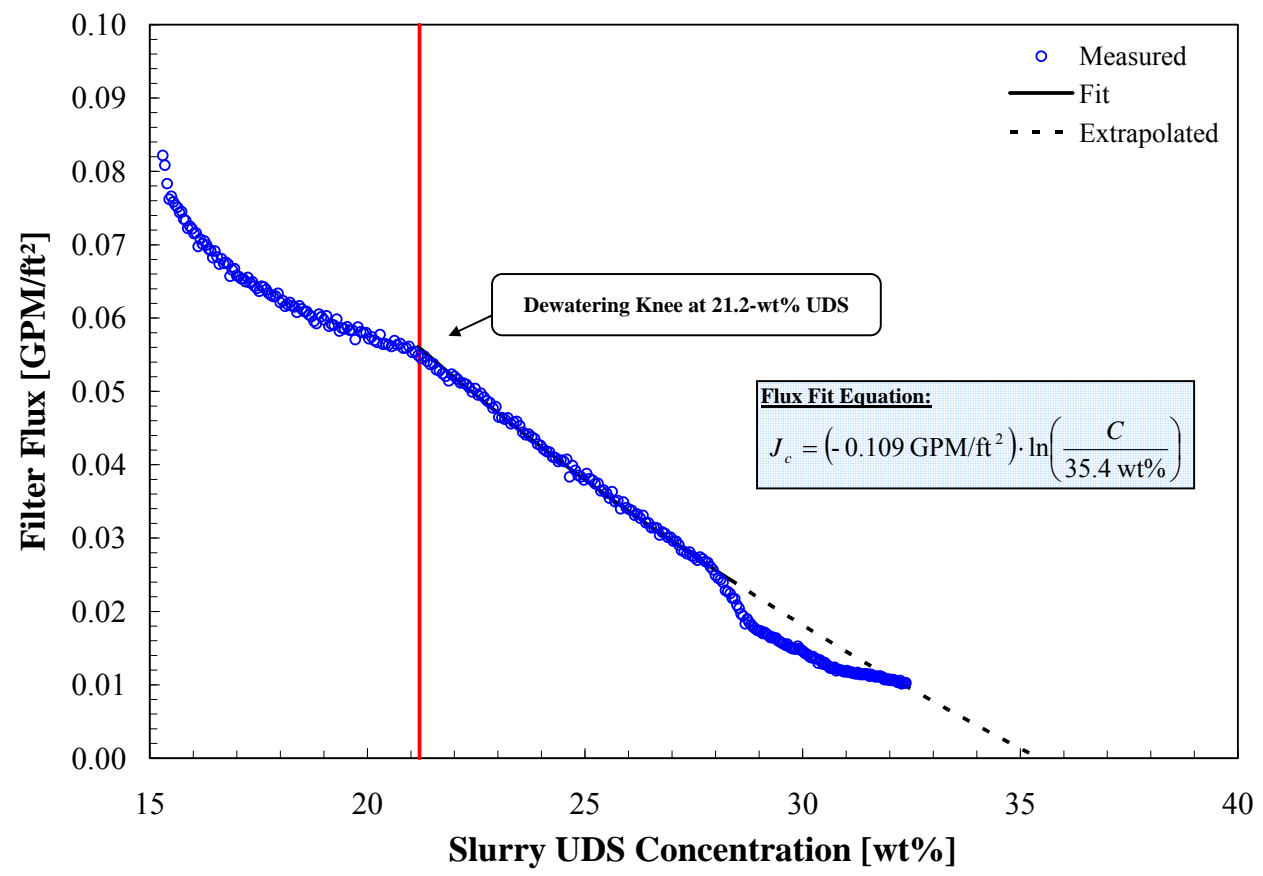

Figure 5.48. Analysis of the Repeat Dewatering Curve Measured During the CUF High-Solids Scaling Test

Table 5.12 summarizes the dewatering curve fitting parameters determined from the PEP and CUF High-Solids Scaling Tests. It also provides a measure of uncertainty in both the mass transfer coefficient and limiting gel concentration. The uncertainty reported for CUF and PEP dewatering parameters represents twice the standard error (as determined by linear regression analysis). These parameters are used to determine the PEP to CUF scaling factor for high-solids dewatering operations shown in Table 5.13. The scaling factor for the mass transfer and limiting gel concentration are $0.97 \pm 0.03$ and $0.96 \pm 0.05$, respectively. Here, the uncertainty is derived from the propagation of standard error through the scaling factor analysis. As before, uncertainty is reported as twice the standard error. Given the uncertainty associated with PEP and CUF operations, both scaling factors appear to statistically similar to 1.0. The majority of uncertainty in the scaling factor derives from uncertainty in the PEP $k$ and $C_{g}$ dewatering parameters (which themselves result from significant variation in PEP AV and temperature during the High-Solids Scaling Test).

Table 5.12. Summary of PEP and CUF High-Solids Dewatering Curve Parameters

\begin{tabular}{lcc}
\hline \multicolumn{1}{c}{ Test Description } & $\mathrm{k}$ & $\begin{array}{c}\mathrm{C}_{\mathrm{g}} \\
{[\mathrm{wP} \%]}\end{array}$ \\
\hline CUF High Solids Test - Initial & $-0.115 \pm 0.001$ & $35.9 \pm 0.5$ \\
CUF High Solids Test - Repeat & $-0.109 \pm 0.001$ & $35.4 \pm 0.8$ \\
CUF Average & $-0.112 \pm 0.001$ & $35.7 \pm 0.5$ \\
PEP High Solids Test & $-0.108 \pm 0.003$ & $34.3 \pm 1.9$ \\
\hline
\end{tabular}


Table 5.13. Summary of PEP to CUF Scaling Factors for High-Solids Dewatering Operations

\begin{tabular}{lc}
\hline \multicolumn{1}{c}{ Scaling Factor } & Value \\
\hline Mass Transfer Coefficient Scaling $\left(S_{k}\right)$ & $0.97 \pm 0.03$ \\
Limiting Gel Concentration Scaling $\left(S_{g}\right)$ & $0.96 \pm 0.05$ \\
\hline
\end{tabular}

Based on the best information currently available, the scaling factor for high-solids dewatering operations appears to be indistinguishable from one. That is, CUF appears to provide a reasonable indication of PEP filter flux performance during high-solids dewatering operations approaching the gel point. Unlike both low-solids scaling tests, the High-Solids Scaling Test for PEP employed a single filter bundle. As discussed in Section 5.1.3, there was concern that CUF scale testing could not be used as a measure of individual PEP performance. However, the current results (specifically, good agreement between the flux parameters characterizing CUF and PEP high-solids dewatering operations) appear to show that the CUF can be used to assess the performance of individual PEP filter elements. This contrasts with the low-solids scaling tests that indicate that CUF provides only an approximate representation of individual PEP filter bundle performance (and that multiple scaling factors must be used to address differences in upstream and downstream filter behavior at PEP). However, there are differences between the low-solids and high-solids scaling tests that may account for this difference in filter behavior:

- Differences in filtration behavior that result from differences in the ratio of slurry solids-to-filter surface area. The test volume employed for CUF testing in the low-solids test was selected to mimic filtration on all five filters in PEP, whereas the CUF test volume employed in the high-solids test was matched to filtration on only one filter in PEP.

- Suspected differences in filter membrane depth fouling (which drive the spread of scaling factors across individual PEP filter bundles) may be less important at high-solids concentration filtration. Typically, dewatering curves are a strong function of the cake layer resistance, which will mask filter membrane resistance.

- Differing slurry composition/chemistry. The low-solids scaling tests employ a low-concentration preleach slurry, whereas the high-solids scaling test employs a leached, washed, and concentrated simulant slurry. These slurries may interact with and foul the filter differently.

- Differences in filtration history. The PEP filters had been exposed to simulant slurries for a much longer period of time at the start of execution of the high-solids test relative to that at the start of the low-solids tests (when the filters were relatively new and freshly cleaned),

A reasonable starting point in addressing these differences would be to first determine the cause of flux divergence in low-solids scaling tests. This may allow a better assessment of why CUF provided a good measure of filtration behavior for a single filter in the high-solids test. 



\subsection{Conclusions}

To assess the scale-up performance of the filtration process for the Hanford Tank Waste Treatment and Immobilization Plant (WTP), the filtration performance of a Hanford tank waste simulant was evaluated at both engineering- and bench-scales. Engineering scale filter performance tests were conducted at the Pretreatment Engineering Platform (PEP) under test plan TP-RPP-WTP-506. ${ }^{(a)}$ Parallel bench-scale tests were conducted under test plan TP-WTP-PEP-044, ${ }^{(b)}$ using the crossflow filtration system (Cells Unit Filter - CUF) located at APEL.

To facilitate an analysis of system scaling, CUF and PEP operations are designed to be equivalent. Both systems use similar filter elements (Mott sintered stainless steel filter tubes of 0.5 -inch inner diameter) taken from the same manufacturer's lot. Both test configurations are similar - a filtration loop is fed from a slurry reservoir/tank with the filtration loop being composed of a slurry pumping system, filtration area, permeate collection and metering systems, heat exchanger (to remove mechanical heat), and filtration loop backpressure valve. Despite these similarities, many operational/configurational differences exist that could yield differences in PEP and CUF scaling. As expected, the most dominant difference is size - as stated previously, PEP has up to 276 times the filter area available in CUF. Other key differences that could limit scaling from CUF to PEP are summarized in Table 6.1.

Table 6.1. Differences Between CUF and PEP

\begin{tabular}{|c|c|c|}
\hline Item & CUF Configuration & PEP Configuration \\
\hline Filtration area & $\begin{array}{l}\text { CUF testing employs a single } 2 \text {-ft-long } \\
\text { filter element comprising a total filtration } \\
\text { area of } 0.262 \mathrm{ft}^{2} \text {. }\end{array}$ & $\begin{array}{l}\text { PEP testing employs multiple filter elements } \\
\text { consisting of a mixture of } 8 \text {-ft-long and } 10-\mathrm{ft} \text { - } \\
\text { long filter elements. Elements are fixed in } \\
\text { bundles containing } 12 \text { filters each. There are five } \\
\text { filter bundles total, comprising a total filtration } \\
\text { area of up to } 72.3 \mathrm{ft}^{2}(276 \text { times that of CUF). }\end{array}$ \\
\hline \multirow[t]{2}{*}{$\begin{array}{l}\text { Process } \\
\text { configuration }\end{array}$} & $\begin{array}{l}\text { (1) Pumping system, (2) heat exchanger, } \\
\text { (3) filter element, (4) backpressure valve, } \\
\text { (5) slurry reservoir. }\end{array}$ & $\begin{array}{l}\text { (1) Pumping system, (2) filtration bundles, } \\
\text { (3) heat exchanger, (4) backpressure valve, } \\
\text { (5) slurry reservoir. }\end{array}$ \\
\hline & Heat exchange precedes the filtration area. & Heat exchange follows the filtration area. \\
\hline Pumps & A single rotary lobe slurry pump. & Two centrifugal slurry pumps operated in series. \\
\hline $\begin{array}{l}\text { Slurry reservoir } \\
\text { mixing system }\end{array}$ & $\begin{array}{l}\text { A single overhead agitator mixes the slurry } \\
\text { reservoir. Additional mixing is provided } \\
\text { by the slurry return from the filtration loop. }\end{array}$ & $\begin{array}{l}\text { Slurry tank mixing is provided by PJMs and air } \\
\text { spargers. Additional mixing is provided by the } \\
\text { slurry return from the filtration loop. }\end{array}$ \\
\hline Filter history & $\begin{array}{l}\text { The filter employed for CUF testing has } \\
\text { been used extensively in simulant } \\
\text { development and testing activities } \\
\text { throughout calendar year } 2008 \text {. }\end{array}$ & $\begin{array}{l}\text { The filter bundles employed for PEP testing are } \\
\text { relatively new and have not been used } \\
\text { extensively. Previous testing is limited to } \\
\text { primarily water functional testing. Contact with } \\
\text { waste simulant slurry is limited. }\end{array}$ \\
\hline
\end{tabular}

(a) GB Josephson, OP Bredt, JK Young, and DE Kurath. 2009. Pretreatment Engineering Platform (PEP) Testing (Phase I). TP-RPP-WTP-506, Rev. 0.4, Pacific Northwest National Laboratory, Richland, Washington.

(b) RL Russell. 2008. Test Plan for the PEP Parallel Laboratory Testing. TP-WTP-PEP-044, Rev. 0.2, Pacific Northwest National Laboratory, Richland, Washington. 
To facilitate the extrapolation of PEP filtration performance to PTF performance, key PEP equipment was made dimensionally or functionally prototypic of the corresponding plant equipment, and PEP operating parameters were chosen to maximize its similarity to the anticipated plant-scale operation. The PEP filtration performance (i.e., flux) and behavior (e.g., depth-fouling, cake formation, entrance effects) are sufficiently prototypic that it is reasonable to assume that the PEP to PTF scale-up factor is 1.

It should be noted that both low-solids tests were also intended to "condition" (i.e., extensively expose to and contact) the filter against the similar slurry solids employed in subsequent tests. For each test run at the PEP, a parallel test was run on the CUF filtration system located at APEL. These tests allow assessment of the PEP to CUF scaling factor for continuous and backpulsed recycle operations and for dewatering operations approaching the slurry gel point. Parallel PEP and CUF tests were performed at similar slurry solids-to-filter surface area ratios (and using filter elements of similar manufacture). The high-solids scaling test was performed at a slurry solids-to-filter area ratio similar to the ratio anticipated for WTP operations; however, both low-solids scaling tests were performed at ratios slightly lower than that anticipated for WTP. With respect to the latter, previous scaling studies in WTP-RPT-168, Rev. 0, suggest that this difference is not expected to impact filtration scaling substantially (Daniel et al. 2009). As such, the low-solids scaling test results likely represent the lower bound of the solids-to-filter area ratio expected in the PTF.

The low-solids scaling tests considered the performance of PEP filtration (as measured through temperature- and TMP-corrected filter flux) against that observed on the CUF test system. The scaling factor was defined as the ratio of PEP filter flux to CUF filter flux. The low-solids scaling tests indicate that for similarly conditioned filters, the CUF flux is comparable to, but slightly under-predicts, the total (area averaged) flux obtained at PEP. The final filter scaling factors based on total PEP flux for lowsolids tests \#1 and \#2 were both $1.1 \pm 0.1$. To provide a conservative estimate for process scaling, a scaling factor of 1.0 is recommended for scaling low-solids filtration operations. A summary of results for the low-solids scaling tests (and key operational parameters) is included in Table 6.2.

Table 6.2. Results for Low-Solids Scaling Tests

\begin{tabular}{lcccc}
\hline Item & CUF & PEP & CUF & PEP \\
\hline Test Description & \multicolumn{2}{c}{ Low-Solids Test \#1 } & \multicolumn{2}{c}{ Low-Solids Test \#2 } \\
Target AV [ft/s] & 15.0 & $15.0 \pm 1.4$ & 15.0 & $15.0 \pm 1.4$ \\
Actual Average AV [ft/s] & $14.9 \pm 0.7$ & 14.8 & $15.0 \pm 0.6$ & 14.8 \\
Target TMP [psid] & 40 & $40 \pm 4$ & 40 & $40 \pm 4$ \\
Actual TMP [psid] & $40.2 \pm 0.8$ & 39.8 & $40.2 \pm 0.4$ & 39.9 \\
Filtration Area [ft ${ }^{2}$ ] & 0.262 & 72.3 & 0.262 & 72.3 \\
Solids-To-Filter Area Ratio [kg/ft ${ }^{2}$ ] & 1.5 & 1.1 & 1.4 & 1.4 \\
Flux Scaling Factor Range (S) & \multicolumn{2}{c}{1.1 to 1.4} & \multicolumn{2}{c}{1.1 to 1.2} \\
Recommended Scaling Factor & \multicolumn{2}{c}{1.0} & \multicolumn{3}{c}{1.0} \\
\hline
\end{tabular}

With regard to the "alternate" goal of filter conditioning, which was to minimize history differences in CUF and PEP by exposing the filter elements to a similar slurry, the conditioning of the filters appears to have been successful from a total (area-averaged) flux stand-point. Specifically, PEP and CUF flux differed substantially (up to 40\%) during the initial run-in period of 12-hrs. In both low-solids scaling tests, a convergence of total filter flux was observed during the second 12-hr period of backpulsed operations, yielding similar CUF and PEP fluxes during the final 12-hrs of operation. Overall, exposure of the filter membrane to slurry solids appears to have reduced potential impacts from differing CUF and 
PEP histories. However, it should be noted that history effects are difficult to distinguish from potential scaling effects. Additionally, frequent backpulsing of the filter appears to be the best driver of filter conditioning. It is speculated that frequent disruption of the protective cake layer allows significant exposure and contact between the filter membrane and slurry solids.

It should be noted that this low-solids operations scaling factor estimate is subject to limitations associated with the test. These limitations derive from the following:

- Divergence of filter flux from individual PEP filter bundles. Both the first and second low-solids tests examined three separate test segments: 1) an initial 12-hr period of continuous (non-backpulsed) recycle filtration, 2) a 12-hr period of backpulsed operations with 24 total backpulses at 30 minute intervals, and 3) a final 12-hr period of continuous (non-backpulsed) recycle filtration. Recycle filtration in all segments employed all five PEP filter bundles. During the first 12-hr segment, filter flux for all five bundles was comparable. However, during the second 12-hr segment, backpulsed operations caused a divergence in the filter flux across each filter. Flux from the upstream bundles was relatively constant throughout backpulsing. In contrast, the downstream filters showed irrecoverable flux loss throughout backpulsing. It is speculated that flux loss on the downstream filters is caused by irreversible depth fouling of the porous filter element during the interim period between cake disruption and cake formation after each backpulse operation. However, this mechanism does not explain why the downstream filters are more susceptible to irreversible fouling. Regardless, the preferential fouling of downstream filters (and the relative immunity of upstream filters with respect to fouling) was observed in both low-solids tests and appears reproducible. The difference in filter flux caused by divergence during backpulsed operations persists into the final 12-hr test segment. At the end of testing, the difference in flux across the filter bundles is still significant-upstream filter flux is 50 to $100 \%$ higher than downstream filter flux.

CUF filter flux appears to fall between the two flux extremes observed in individual PEP filter bundles. As a result, PEP to CUF scaling factors based on individual PEP filter fluxes range from $\sim 0.7$ up to $\sim 1.6$. This indicates that CUF filter flux provides an inexact representation of the flux performance of individual PEP filter bundles (for the low-solids scaling tests). That being stated, the difference in CUF and individual PEP bundle performance is not great. CUF provides an order of magnitude approximately of PEP filter bundle flux and an approximate representation of the flux time dependency. Additionally, when PEP filter flux is considered on a total (i.e., area-averaged) flux basis, the cold-CUF provides an excellent representation of PEP performance (with scaling factors close to 1.0 for conditioned filters). Thus, the bench-scale CUF provides an accurate measure of PEP filter flux magnitude and dynamics when the flux across all filters was considered for conditioned filters. The test results for the low-solids scaling test also indicate that CUF filter flux provides a conservatively low estimate of flux for unconditioned filters.

While CUF appears to provide an accurate measure of PEP filter performance, the underlying concern is that flux divergence observed during backpulsing was not expected and is currently not understood. Further study of the mechanisms causing PEP flux divergence is recommended to allow better assessment of their potential impacts on scaling analyses. 
- Differences in the state of PEP and CUF initial filter conditioning. The recommended low-solids scaling factor of 1.0 is based on the assumption of similarly conditioned filters. Application to unconditioned filters may require scaling factors different than one. In the low-solids tests presented in the current report, the CUF significantly under predicted the PEP flux during the first 12-hr test segment of the low-solids tests (where CUF and PEP filters are relatively unconditioned by the simulant slurry). The scaling factors associated with the initial 12-hr test segment were $1.4 \pm 0.2$ and $1.2 \pm 0.1$ for first and second low-solids scaling tests, respectively. Filter conditioning reduced this flux discrepancy — at the end of testing, the scaling factors were both $1.1 \pm 0.1$. In short, the best agreement between CUF and PEP total filter flux for the low-solids scaling tests is achieved only after the filters have been conditioned (i.e., fouled) against a similar waste simulant. To enable better scaling and comparison (especially for unconditioned filters), an evaluation of the effects of nitric and oxalic acid cleaning on the performance of the filter elements is recommended.

- Insufficient process test time to achieve filtration steady-state. For both low-solids scaling tests performed on the PEP and CUF filtration systems, the 12-hr test segments were insufficient to reach a process steady state (or even to assess the existence/value of a steady state flux). This limitation impacts both CUF and PEP filtration, and as such, all filtration results discussed in this report are subject to further time-dependent decay. The lack of a filtration steady state (and continued decline of filter flux throughout the test) does not appear to impact agreement (and subsequent scaling factor analyses) of total PEP and CUF filter fluxes - the scaling factors observed for conditioned filters in the low-solids scaling tests showed little time-dependence and were close to 1.0. However, continued flux decay throughout the test introduces uncertainty with respect to PEP and CUF scaling over time frames longer than those tested in the current report. An evaluation of long-term (i.e., much greater than $36 \mathrm{hrs}$ ) filter flux dynamics is recommended to assess their potential impacts on scaling of filtration performance.

High-solids scaling factor analysis considered scaling in terms of the parameters characterizing filtration dewatering performance at concentrations approaching the limiting gel concentration. These parameters are 1) the dewatering mass transfer coefficient $(k)$ and 2$)$ the slurry limiting gel concentration $\left(C_{g}\right)$. Two separate scaling factors were defined - the first is the ratio of PEP $k$ to CUF $k$, and the second is the ratio of PEP $C_{g}$ to CUF $C_{g}$.

Analysis of PEP and CUF high-solids dewatering curves indicates scaling factors of $0.97 \pm 0.03$ and $0.96 \pm 0.05$ for both $k$ and $C_{g}$, respectively. These results indicate that the high-solids filtration performance CUF and PEP are indistinguishable from one another. Based on the best information currently available, the scaling factor for high-solids dewatering operations appears to be one. That is, CUF appears to provide a reasonable indication of PEP filter flux performance during high-solids dewatering operations approaching the gel point. A summary of results for the high-solids scaling test (and key operational parameters) is included in Table 6.3. 
Table 6.3. Results for High-Solids Scaling Tests

\begin{tabular}{lcc}
\hline Item & CUF & PEP \\
\hline Test Description & \multicolumn{2}{c}{ High Solids Test } \\
Target AV [ft/s] & 15.0 & $15.0 \pm 1.4$ \\
Actual Average AV [ft/s] & $15.0 \pm 0.1$ & 14.7 \\
Target TMP [psid] & 40 & $40 \pm 4$ \\
Actual TMP [psid] & $41 \pm 1$ & 39.8 \\
Filtration Area [ft ${ }^{2}$ trea Ratio [kg/ $\left.\mathrm{ft}^{2}\right]$ & 0.262 & 15.7 \\
Solids-To-Filter Area & 14.5 & 13.9 \\
Dewatering Mass Transfer Coefficient [GPM $\left./ \mathrm{ft}^{2}\right]$ & $-0.112 \pm 0.001$ & $-0.108 \pm 0.003$ \\
Limiting Gel Concentration [wt $\%$ ] & $34.3 \pm 1.9$ & $35.7 \pm 0.5$ \\
Mass Transfer Scaling Factor $\left(\mathrm{S}_{\mathrm{k}}\right)$ & \multicolumn{2}{c}{$0.97 \pm 0.03$} \\
Limit Gel Concentration Scaling Factor $\left(\mathrm{S}_{\mathrm{g}}\right)$ & \multicolumn{2}{c}{$0.96 \pm 0.05$} \\
\hline Recommended Scaling Factor & \multicolumn{2}{c}{1.0} \\
\hline
\end{tabular}





\subsection{References}

Daniel RC, JM Billing, KJ Cantrell, ML Luna, RA Peterson, ML Bonebrake, RW Shimskey, and LK Jagoda. 2009. Characterization of Filtration Scale-Up Performance. WTP-RPT-168, Rev. 0, PNNL18117, Pacific Northwest National Laboratory, Richland, Washington.

Geeting JGH, RT Hallen, LK Jagoda, AP Poloski, RD Scheele, DR Weier. 2003. Filtration, Washing, and Caustic Leaching of Hanford Tank AZ-101 Sludge. WTP-RPT-043, Rev. 1, PNWD-3206 Rev. 1, Pacific Northwest National Laboratory, Richland, Washington.

Rapko BM, GJ Lumetta, JR Deschane, and RA Peterson. 2007. Process Development for Permanganate Addition During Oxidative Leaching of Hanford Tank Sludge Simulants. WTP-RPT-164, Rev. 0, PNNL-16794, Pacific Northwest National Laboratory, Richland, Washington.

Russell RL, RA Peterson, HD Smith, DE Rinehart, PM Aker, and EC Buck. 2009a. Development and Characterization of Boehmite Component Simulant. WTP-RPT-184, Rev. 0, PNNL-18176, Pacific Northwest National Laboratory, Richland, Washington.

Russell RL, Peterson RA, DE Rinehart, HD Smith. 2009b. Development and Characterization of Gibbsite Component Simulant. WTP-RPT-176, Rev. 0. PNNL-18013. Pacific Northwest National Laboratory, Richland Washington.

Russell RL, JM Billing, RA Peterson, DE Rinehart, and HD Smith. 2009c. Development and Demonstration of Ultrafiltration Simulants. WTP-RPT-183, Rev. 0, PNNL-18090, Pacific Northwest National Laboratory, Richland, Washington. 



\section{Distribution}

No. of

Copies

OFFSITE
No. of

Copies

ONSITE

12 Pacific Northwest National Laboratory

J. M. Billing

$\mathrm{P} 7-25$

J. R. Bontha

$\mathrm{P} 7-25$

C. F. Brown

$\mathrm{P} 7-22$

R. C. Daniel

$\mathrm{P} 7-22$

P. W. Eslinger

K6-50

B. D. Hanson

P7-27

J. L. Huckaby

$\mathrm{H} 4-02$

N. K. Karri

K5-22

M. L. Kimura

K6-28

D. E. Kurath

K3-52

M. J. Minette

P7-25

Project File (1)

K3-52

Information Release (pdf)

3 Bechtel National, Inc.

WTP R\&T Docs (2)

$\mathrm{H} 4-02$

S. Barnes

H4-02

2 Office of River Protection

R. A. Gilbert

H6-60

J. A. Reddick

H6-60

Distr. 1 


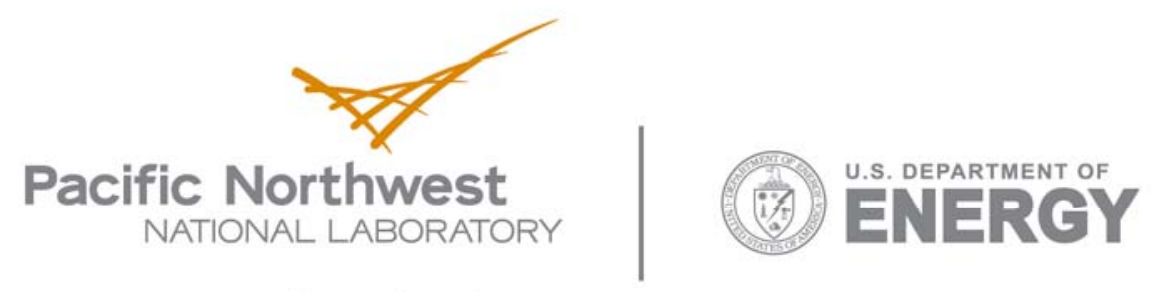

902 Battelle Boulevard

P.O. Box 999

Richland, WA 99352

1-888-375-PNNL (7665)

www.pnl.gov 